LAWRENCE LIVERMORE N A T IO N A L LABORATORY

Final report on isotope tracer investigations in the Forebay of the Orange County groundwater basin.

M.L. Davisson, G.B. Hudson, J.F.Clark, G. Woodside, R. Herndon

January 8, 2004 
This document was prepared as an account of work sponsored by an agency of the United States Government. Neither the United States Government nor the University of California nor any of their employees, makes any warranty, express or implied, or assumes any legal liability or responsibility for the accuracy, completeness, or usefulness of any information, apparatus, product, or process disclosed, or represents that its use would not infringe privately owned rights. Reference herein to any specific commercial product, process, or service by trade name, trademark, manufacturer, or otherwise, does not necessarily constitute or imply its endorsement, recommendation, or favoring by the United States Government or the University of California. The views and opinions of authors expressed herein do not necessarily state or reflect those of the United States Government or the University of California, and shall not be used for advertising or product endorsement purposes.

This work was performed under the auspices of the U.S. Department of Energy by University of California, Lawrence Livermore National Laboratory under Contract W-7405-Eng-48. 


\title{
Final Report on Isotope Tracer Investigations in the Forebay of the Orange County Groundwater Basin.
}

\author{
M.L. Davisson \\ G.B. Hudson \\ Lawrence Livermore National Laboratory \\ J.F. Clark \\ University of California, Santa Barbara \\ G. Woodside \\ R. Herndon \\ Orange County Water District
}

December, 2003 


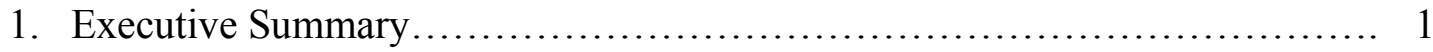

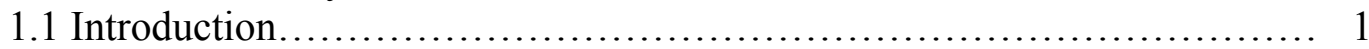

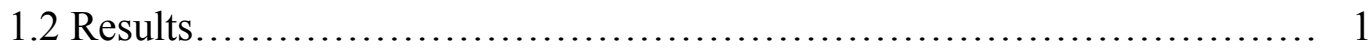

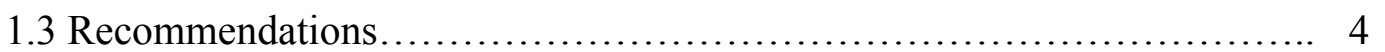

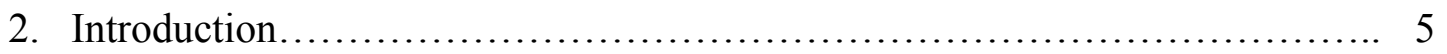

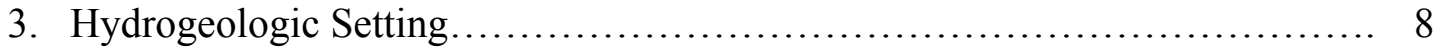

4. Research Approach...................................................... 12

5. Analytical Methods........................................................ 14

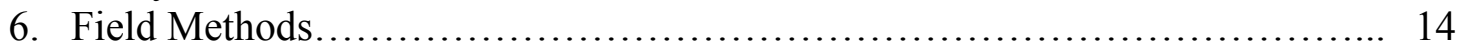

7. Results From the Environmental Isotope Characterization and Age-Dating...... 14

7.1 Age-Dating Results................................................ 14

7.1.1 $\left({ }^{3} \mathrm{H}\right)$ Tritium Results............................................ 15

7.1.2 Noble Gas Results............................................. 36

7.1.3 Calculated Ages............................................. 39

7.2 Stable Isotope Results.......................................... 44

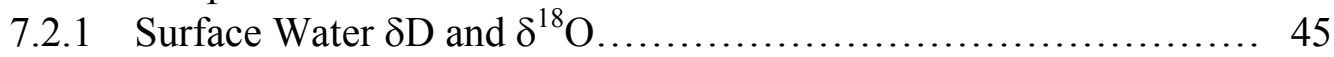

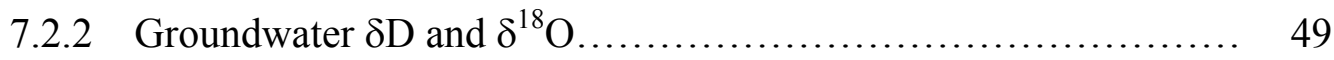

7.2.2.1 Wells Adjacent to or Recharged Exclusively by the SAR..... 49

7.2.2.2 Wells Downgradient of Anaheim Lake and Kraemer Basin...... 50

7.3 Radiocarbon and Carbon-13 Results................................... 52

8. Research Results on the Artificial Tracer Experiments...................... 53

8.1 1996 Anaheim Lake Recharge Experiment............................ 53

8.1 .1 Recharge Condition and Setup................................ 53

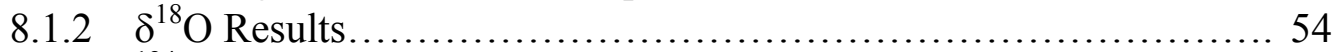

8.1.3 ${ }^{124} \mathrm{Xe}$ Results........................................................... 66

8.2 1998 Anaheim Lake and Kraemer Basin Recharge Experiment............. 69

8.2.1 Recharge Condition............................................... 69

8.2.2 Kraemer Basin Recharge...................................... 70

8.2.2.1 North Flowpath $\delta^{18} \mathrm{O}$ and Xe Isotope Results.................... 72

8.2.2.2 South Flowpath $\delta^{18} \mathrm{O}$ and Xe Isotope Results.................... 73

8.2.2.3 $\delta^{18} \mathrm{O}$ and $\mathrm{Xe}$ Isotope Results from Wells Adjacent to Kraemer Basin. 74

8.2.3 Anaheim Lake Recharge....................................... 77

8.31998 Santa Ana River Tracer Study ................................... 78

9. Discussion on Sources of Recharge .................................... 82

10. Discussion on Groundwater Ages.................................... 85

10.1 General Uncertainties in Age Determinations.......................... 85

10.2 Hydrologic Implications of Age Determinations...................... 87

11 Discussion of Flowpaths and Flow Rates.................................. 91

11.1 Co-mingling of Different Recharge Sources in Forebay Groundwater..... 91

11.2 Tracer Travel Times.............................................. 93

12 Groundwater Dispersion and Dilution................................... 98

13 Summary and Conclusions............................................ 102

14 Recommendations................................................. 103

15 Acknowledgements................................................ 104

16 References............................................................. 104 
17 Appendix 1 General Discussion on Use of Isotope Measurements in Groundwater Characterization......................................................... 107

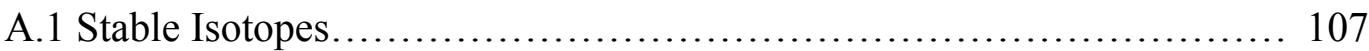

A.2 Tritium-Helium-3 Age Dating.................................... 108

A.3 Noble Gas Abundance.............................................. 110

A.4 Sulfur Hexafluoride................................................. 111

A.5 Radiocarbon and Carbon-13 ................................... 112

18 Appendix 2 Analytical Methods......................................... 113

19 Appendix 3 Development of Sampling Protocols for Noble Gases from Westbay Wells................................................................ 114

20 Appendix References.............................................. 115 
TABLES

Page

Table 1. Isotope Data, Ages, and Recharge Temperatures Calculated for Groundwater in the Forebay .......................................................... 16

Table 2. Equilibrium Solubility of Noble Gases in Water at $35^{\circ} \mathrm{C}$ and at Sea Level 36

Table 3. Annual Imported Water Volume Used for Recharge in Forebay........... 45

Table 4. Xenon Tracer Results from 1996 and 1998 Recharge Experiments........ 54

Table 5. $\mathrm{SF}_{6}$ Tracer Results................................................ 80

Table $6 .{ }^{3} \mathrm{H}$ Fallout Inventory Estimates for Forebay Recharge................... 83

Table 7. Transport Rates Derived from $\mathrm{SF}_{6}$ and Xenon Isotope Results............. 93 
FIGURES

Figure 1a-b. Map of Orange County Groundwater Basin and Cross Section...... 5

Figure 2a-d. Map View of Forebay, Groundwater Levels, and Cross Sections...... 10

Figure 3. Colorado River and Santa Maria ${ }^{3} \mathrm{H}$ Records......................... 35

Figure 4. SAR Water Temperature Records................................ 36

Figure 5. ${ }^{20} \mathrm{Ne}$ versus ${ }^{4} \mathrm{He}$ plot............................................... 37

Figure 6. Average ${ }^{3} \mathrm{H}_{-}^{3} \mathrm{He}$ Age Contours in the Forebay Groundwater............. 39

Figure 7. Recharge Year versus initial ${ }^{3} \mathrm{H}$ Content............................... 40

Figure 8a-b. Age and $\delta^{18} \mathrm{O}$ versus depth in Wells A-42 and A-43 ................. 42

Figure 9. $\delta \mathrm{D}$ versus $\delta^{18} \mathrm{O}$ Plot of Forebay Groundwater and Surface Water........ 43

Figure 10. $\delta^{18} \mathrm{O}$ versus Time in SAR and Anaheim Lake........................... 44

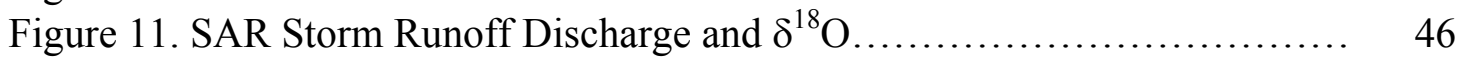

Figure 12. Depth Profiles of Dissolved Oxygen, EC, and T ${ }^{\circ} \mathrm{C}$ Behind Prado Dam 47

Figure 13. $\delta^{18} \mathrm{O}$ versus Depth in Recharge Basins............................ 47

Figure 14a-c. $\delta^{18} \mathrm{O}$ Time Series for Forebay Groundwater and Surface Water..... 50

Figure 15a-b. 1996 Artificial Recharge Experiment $\delta^{18}$ O Results................... 53

Figure 16a-c. Model of Xenon Isotope Abundance in Recharge Basins.............. 66

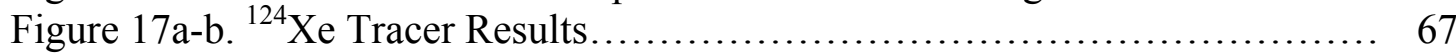

Figure 18. Map of North, South, and Anaheim Lake Flowpaths.................... 70

Figure 19a-b. 1998 Artificial Recharge Experiment North Flowpath Results....... 71

Figure 20a-b. 1998 Artificial Recharge Experiment South Flowpath Results....... 72

Figure 21a-b. 1998 Recharge Experiment Anaheim Lake Flowpath Results........ 74

Figure 22a-b. 1998 Artificial Recharge Experiment AMD-10 Results................ 75

Figure 23a-b. Comparison of Results from 1996 and 1998 Recharge Experiments 76

Figure 24. Map of $\mathrm{SF}_{6}$ Tracer Points of Introduction into SAR .................... 78

Figure 25. $\mathrm{SF}_{6}$ Concentrations Measured in SAR............................ 79

Figure 26a-b. $\mathrm{SF}_{6}$ Results in Forebay Groundwater........................... 79

Figure 27. Comparison of ${ }^{3} \mathrm{H}$ Records between Groundwater and Surface Water... 84

Figure 28a-c. Map Contours of ${ }^{3} \mathrm{H}_{-}^{3} \mathrm{He}$ Ages at Different Depths in Forebay..... 87

Figure 29a-b. Cross Section of ${ }^{3} \mathrm{H}^{-}{ }^{3} \mathrm{He}$ Ages in Forebay.......................... 89

Figure 30. Areas Recharged by Colorado River and Xenon and $\mathrm{SF}_{6}$ Tracers..... $\quad 91$

Figure 31. Plot of Distance versus Tracer Arrival Time.......................... 92

Figure 32. Contour Map of Tracer Arrival Time............................ 94

Figure 33a-b. Xenon Tracer Dispersion in Wells Downgradient of Kraemer Basin 97

Figure 34. Map of Tracer Plumes in Forebay .................................. 98

Figure 35. Plot of First Tracer Arrival versus Center of Mass Arrival............. 99

Figure 36. Plot of Tracer Concentration and Peak Half-Width versus Distance...... 100 


\section{Executive Summary}

\subsection{Introduction}

California is currently faced with some critical decisions about water resource infrastructure development in highly urbanized regions, whose outcome will dictate the future long-term viability of plentiful water. Among these is developing and safely implementing the reuse of advanced treated waste water. One of the most reliable strategies for this water resource is its indirect reuse via groundwater recharge and storage, with particular emphasis on supplementing annual water demand or during drought relief.

The Orange County Water District (District) is currently implementing the first phase of a large-scale water reuse project that will advance-treat up to 60 million gallons per day of waste water and recharge it into existing percolation basins in the Forebay region of the Orange County groundwater basin. In order for the District to protect public health, the fate and potability of this recharged waste water needs to be understood. In particular, the direction and rates of flow into underlying aquifers need to be characterized so that changes in water quality can be quantified between the recharge basins and points of production. Furthermore, to ensure compliance to California Department of Health Services (DHS) draft regulations, the direction and rate of recharged waste water from these basins need to be understood to sufficient detail that small mixtures can be delineated in monitoring and production wells. Under proposed DHS guidelines, consumptive use of recycled water is permissive only if its residence time in an aquifer exceeds a specified six-month time-frame. DHS guidelines also limit the percentage of recycled water at production wells. However, attaining such detail using current hydrogeological and computer-assisted modeling tools is either cost-prohibitive or results in uncertainties too large to achieve regulatory confidence.

To overcome this technical barrier, the District funded Lawrence Livermore National Laboratory (LLNL) from 1995-2001 to directly measure groundwater ages and perform two artificial tracer studies using isotope methods to quantify flowpath directions, groundwater residence times, and the rate and extent of recharge water and groundwater mixing. In addition, Jordan Clark at University of California, Santa Barbara also performed an artificial tracer experiment using sulfur-hexafluoride, whose results have been integrated into the LLNL findings.

\subsection{Results}

In 1995 and 1996, LLNL demonstrated in a feasibility study that the tritium-helium-3 dating method could provide age constraints of plus or minus one year in Forebay groundwater. Groundwater ages dated by this method ranged from zero to greater than 40 years, having an average age of approximately 14 years among the groundwater sampled and measured. Groundwater ages increased with distance from the recharge basins and with depth in the Forebay aquifers. Groundwater flow was rapid near the recharge basins, where groundwater ages less than 2 years old occurred over a two-mile downgradient distance.

Initial investigation also showed that the stable isotope values of oxygen-18 and deuterium in some Forebay groundwater wells varied significantly over several weeks, reflecting different recharge pulses and water sources. For example, the oxygen-18 abundance in Colorado River water was consistently lower than Santa Ana River water, 
which facilitated accurate mixing calculations between these two sources with a precision of plus or minus 10 percent.

In the western Forebay region, there was no temporal variation in oxygen-18 over several months. However, lower oxygen-18 abundances was common in this area and were attributed to mixtures of Colorado River water recharged in Anaheim Lake between 1966 and 1994 based on age dating.

The precision in the tritium-helium-3 age determinations was adequate for the purpose of supporting hydrogeologic characterization of Forebay aquifers and identifying major flow paths from the recharge basins. The ages were not precise enough to delineate in wells small mixtures of recharge water less than 1 year old, which is a critical element in the draft DHS criteria. Furthermore, the ages could not determine if groundwater was recharged from off channel basins or from the SAR.

In order to distinguish recharge origin and small mixtures of young water in wells, artificial tracers were introduced into the recharge basins and the SAR and tracked for over two years in monitoring and production wells in the Forebay. These tracers were safe to human health and easily detected at very low concentrations.

A combined Colorado River-xenon isotope tracer experiment was initiated in October, 1996, in which 6300 acre-ft of Colorado River water was recharged in Anaheim Lake. An isotopically-enriched xenon-124 tracer was introduced into the Colorado River 10 days after percolation started. Another tracer experiment was initiated in October, 1998, in which approximately 9500 acre-ft of Colorado River water was diverted and recharged simultaneously into Anaheim Lake and Kraemer Basin, and, respectively, xenon-129 and xenon-136 tracers were individually added. Also in 1998, the SAR was spiked with a sulfur hexafluoride tracer over 15 days in order to facilitate tracking of SAR recharge through the subsurface.

The low oxygen-18 abundance of the recharged Colorado River water provided an additional tracer of groundwater flow through the subsurface. For both the 1996 and 1998 recharge basin tracer experiments, mixtures of Colorado River water in groundwater could be reliably measured at about 10 to 20 percent contribution. The Colorado River water could be traced in groundwater for approximately 8 to 10 months after recharge began, corresponding to approximately one mile downgradient flow before groundwater dispersion made isotope values of Colorado River mixtures difficult to distinguish from the natural isotope variation in SAR water.

The xenon isotope measurements for both 1996 and 1998 recharge basin tracer experiments provided a robust tracer, where one to two percent mixtures of Colorado River water labeled with a xenon isotope could be easily detected in groundwater over four years after recharge and up to two miles downgradient. A good illustration of the benefits of a multi-isotope tracer approach was when xenon-124 was observed in deep production well A-44 below Anaheim Lake at approximately 3\% of the recharge concentration around 160 days after the tracer was introduced into Anaheim Lake. However, the tritium-helium age measured in A-44 was 11 years. The low-level detection of xenon-124 in A-44 was consistent with spinner log tests performed in 1997 that indicated $>60 \%$ of the groundwater production from this well was from shallow depth intervals.

The isotope tracer experiments elucidated details on groundwater flowpath trajectories from points of recharge to groundwater wells. Recharge originating from Anaheim Lake 
was observed 15-30 days after recharge at monitoring and production wells several hundred feet from the lakeshore and greater than 200 feet deep. An approximately 100\% turnover in groundwater within 30 days in one well (AMD-9 level 1) provided an excellent monitoring point for quantifying short-term water quality changes resulting from basin recharge.

The downgradient movement of Anaheim Lake recharge, beyond adjacent shallow monitoring wells, was apparently limited at shallow depths and more commonly observed in deeper zones of multi-level monitoring wells. For example, the xenon tracers were only observed in shallow wells AM-44 and KBS-4 up to 2500 feet downgradient and approximately 120 days after tracer introduction. However, Anaheim Lake recharge water was observed up to four years after recharge in deep aquifer zones ( $>750$ feet) at multi-point monitoring wells 4000 feet downgradient. The tracer data suggested that in the immediate Forebay region Anaheim Lake recharge replenished aquifer zones deeper than those recharged by Kramer Basin.

Tracer results showed recharge originating from Kraemer Basin flowed in a coneshaped pattern in shallow aquifers. Xenon-labeled groundwater flow was rapid, reaching production wells SCWC-PLJ2 and A-26 over one mile downgradient in less than one year. Groundwater recharge from Kraemer Basin was diluted by other recharge sources during downgradient flow. Kraemer Basin recharge flowing southwestward was suspected to mix with SAR recharge, while recharge flowing northwestward was mixed with older groundwater either originating from the Yorba Linda sub-basin or SAR recharge upgradient from Anaheim Lake.

Santa Ana River water labeled with sulfur hexafluoride and recharged into Forebay groundwater was readily detected at trace levels in monitoring and production wells in the Forebay. Large uncertainty in the sulfur hexafluoride concentration at the point of recharge prevented accurate mixing calculations in groundwater. The concentration of sulfur hexafluoride in the river channel varied over an order of magnitude, due to airwater exchange in turbulent zones.

Results from the sulfur hexafluoride tracer experiment that tracked SAR recharge showed that groundwater flow for the initial approximately 60 days was primarily perpendicular to the river channel. Afterward the recharged SAR water followed a westwardly course along the hydraulic gradient and was detected over two years later in wells greater than 1.5 miles downgradient. The arrival of sulfur hexafluoride-tagged SAR water in most wells was gradual but persistent over many months, suggesting a high degree of groundwater dispersion.

An inter-comparison of tracer arrival times and travel distances for all wells measured in the Forebay revealed that the maximum velocity of groundwater is approximately uniform throughout this region at around $8000 \mathrm{feet} / \mathrm{yr}$. The exception is an area adjacent to SAR at the point of its southward bend. Here, the groundwater velocity was more than a factor of two lower then the remainder of the Forebay, presumably due to impedance from the Peralta Hills fault.

In only one case after two years of monitoring for tracer movement in the groundwater was there evidence for mixing among the different artificial tracers introduced into the Forebay. In the shallowest level of monitoring well AMD-10, both the xenon-129 and xenon-136 was observed in the same samples, indicating this aquifer level was recharged simultaneously by both Anaheim Lake and Kraemer Basin. Sulfur hexafluoride was not 
observed in groundwater that also had a xenon tracer. The lack of observed tracer comingling is likely due to groundwater transport being dominated by longitudinal advection and dispersion. When water levels in both the SAR and the recharge basins are essentially equal, groundwater mixing lateral to flow direction is limited.

\subsection{Recommendations}

Based on the results and interpretations outlined in this report, some key recommendations are presented for future recharge monitoring and basin evaluation. With anticipated completion of the Groundwater Replenishment System, increased percolation in Kraemer Basin will lead to increased mean linear groundwater velocity downgradient and ultimately a reduction in the age of groundwater produced from nearby production wells. Changes in groundwater velocity can be determined by frequently monitoring groundwater in wells such as SCWC-PLJ2, A-26 and AM-14 by exploiting changes in recharge water character, such as changes from SAR to Colorado River source water.

It is recommended to continue high frequency monitoring (once a month) of nearby wells such as AMD-9 level 1 and OCWD-KB1. These wells have proven reliable indicators of approximately one-month old recharge water with no dilution from their respective recharge basins. These wells will be key monitoring points for potential changes in water quality parameters important to the District and that are relevant to Department of Health Services issues, for example dissolved organic carbon.

It is further recommended to continue high frequency water quality monitoring of wells SAR-8 level 1 and OCWD-LV1, which should reveal important water quality transitions with SAR recharge, such as DOC removal.

In the event that studies of recharge from the Santiago Pits becomes important to District, it is recommended that a sulfur-hexafluoride tracer be used for determining groundwater transport rates and recharge water/groundwater mixing in nearby wells. Although sulfur-hexafluoride loss was large during the SAR recharge study, a release at a relatively deep depth in the Santiago Pits should limit atmospheric loss and provided a quantifiable source concentration.

Xenon isotope measurements should continue to a limited degree for wells downgradient of A-29 and AM-15. Peak concentration should be detectable at or above 0.5 percent of the source concentration for potentially two more years. However, decreasing groundwater flow velocities downgradient, because the groundwater basin widens, may increase mixing rates, and the detectable signal may decrease below detection limits sooner.

Lastly, District should make a special effort to further enhance their knowledge of the groundwater basin by integrating the detailed results of this recharge study with the recently completed groundwater age survey completed under the auspices of the Ambient Groundwater Monitoring Program sponsored by the State Water Resources Control Board. Data integration will produce a basin-wide interpretation of groundwater transport rates, correlation of groundwater quality to time since recharge, interpretation of processes governing vertical transport, and evidence for age and character of unutilized deep groundwater sources. 


\section{Introduction}

In the past 25 years urban growth in water-challenged regions of the US has solidified interest in potable reuse of reclaimed water. Most commonly this is practiced by indirect potable reuse through groundwater recharge of advanced treated waste water (NRC,1998). Indirect reuse serves a dual purpose of 1) increasing water supply and augmenting annual demand, and 2) enhancing water quality by exploiting the natural attenuating properties of aquifer sediments, which in turn reduces public health risks from potential exposure to pathogens and contaminants. For years, the Orange County Water District (District) has developed and applied advanced treatment technologies to waste water and has injected it into a subsurface seawater intrusion barrier project. The District has continued to develop alternatives to imported water supplies by increasing water reuse practices and maximizing local water sources. In particular, the District has initiated a plan to build a 70 million gallon per day advanced water recycling plant and transport its product water to surface spreading facilities located in the Forebay region of Orange County (Fig. 1). These spreading facilities were developed and are maintained to augment recharge of the groundwater basin.

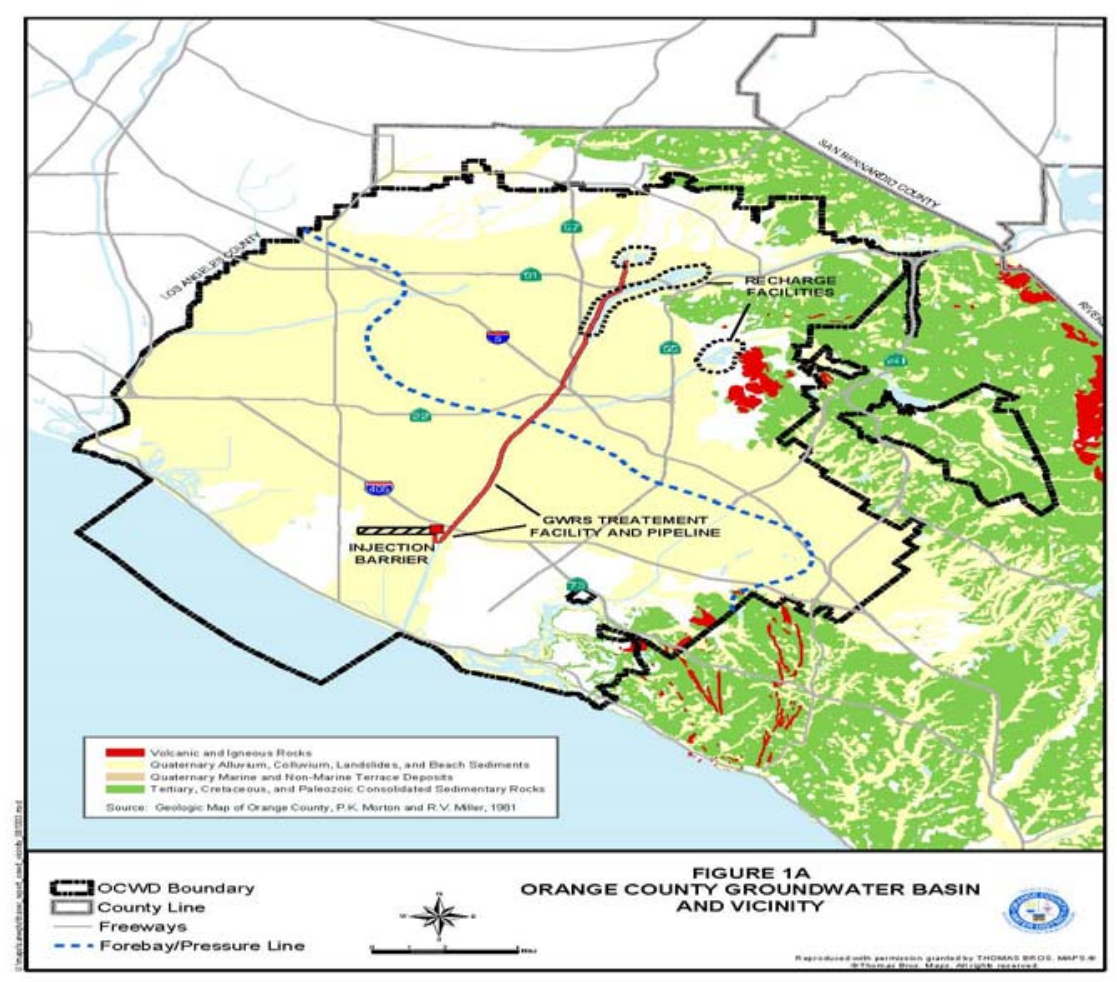

Figure 1. a) Map view of the Orange County groundwater basin with average groundwater levels. The basin is maintained by the Orange County Water District. 


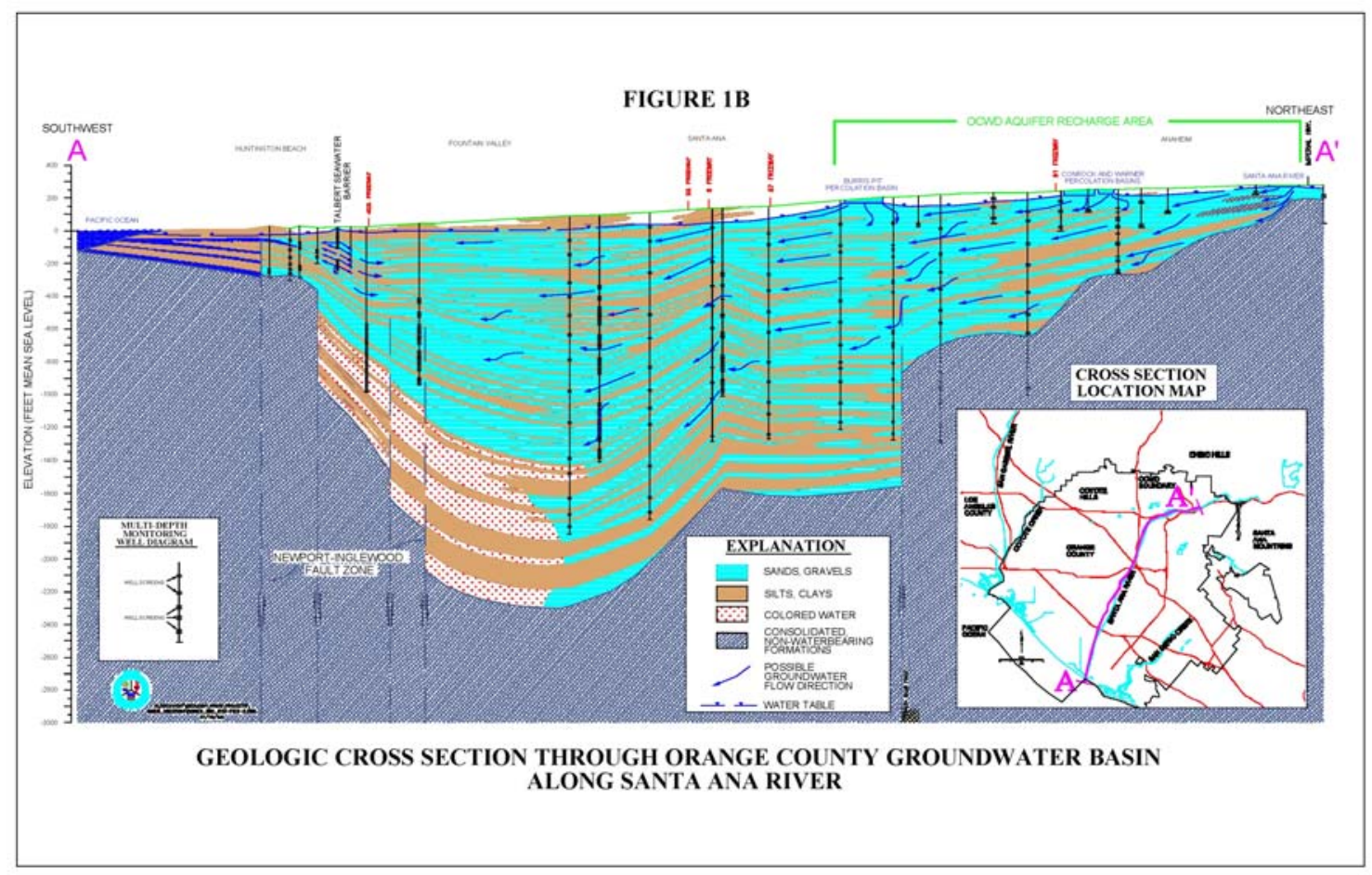

Figure 1. b) Simplified cross-section of basin illustrated trough shape and inter-layered sediments form aquifers and aquitards.

The spreading basins and the SAR recharge a total of greater than 200,000 acre- $\mathrm{ft}$ annually, but currently the source water is derived mostly from diverted SAR, with periodic diversions of Colorado River, and mixtures of State Water Project water. Some of the potable production wells within the Forebay produce water recharged from these basins. In order for the District to protect public health, the fate and potability of this recharge needed to be understood. In particular, the direction and rates of flow into underlying aquifers needed to be characterized so that changes in its water quality could be quantified between the recharge basins and points of production.

However, the District's plans to divert and recharge reclaimed water from the proposed 70 million gallon per day recycling plant, requires regulatory approval from the California Department of Health Services (DHS). Approval is based on draft criteria for groundwater recharge of recycled waste water formulated by DHS (DHS, 2001). One of the main tenets of these criteria is the requirement that reclaimed water intentionally recharged into an aquifer must reside in the subsurface six to nine months before any subsequent domestic consumption, depending on several factors such as local hydrogeologic conditions and recharge mechanisms. Another part of the draft DHS criteria is limits on the percentage of recycled water at production wells used for potable supply. In order to demonstrate with minimal uncertainty that this regulatory criteria is achieved, advanced tools for measuring the rates of groundwater recharge, flow, and mixing in complex aquifers systems need to be available. In most cases, however, available standard hydrogeologic approaches, such as drill log data, pump tests, and geophysical methods, are costly and incapable of providing the necessary detail to meet the stringent guidelines required by DHS. In particular, the residence time requirement 
for recharged water before potable reuse offers a unique challenge to the groundwater sciences.

It is interesting to note that previous studies in the water quality effects of recharged waste water are set in or portrayed as simplified groundwater flow systems (e.g. Barber et al., 1988; Ding et al., 1999; Drewes and Fox, 1999). Laboratory based studies, whose particular focus may be elucidating the fate of pathogens or synthetic chemicals were usually conducted under one-dimensional flow conditions in isotropic media. Directly translating results from these type of studies to complex field conditions often poses difficult challenges in order to explain observed conditions or to predict net effects (Wild and Reinhard, 1999; Maxwell et al., 2003). In field-based studies, fate and transport investigations of waste water contaminants have either been in settings with highly uniform aquifers (e.g. Barber et al., 1988), or in the case of heterogeneous aquifers, the groundwater flow has been simply represented by potentiometric surfaces (e.g Ding et al., 1999). However, a potentiometric surface or a water quality sample measured in complex heterogeneous media actually consists of a hierarchy of flow paths with different source terms and transport histories (e.g. Tompson et al., 1999). It is the flow path of youngest age that is typically of greatest regulatory concern.

The difficulty of accurately representing heterogeneous groundwater flow rests on the need for high density data in order to capture the range of hydrological parameters inherent in heterogeneous environments. Well drilling is expensive and prohibitive for complex geologic deposits typically found in alluvial terrains such as in the Forebay region. Computer modeling designed to represent the details of heterogeneity requires large, and commercially unavailable computational power, whose result still demands data with which to calibrate and an observational validation.

The direct measurement of the groundwater age through the use of isotopic measurements provides an attractive option for potable reuse studies and evaluating regulatory compliance. In particular, providing that these measurements had the required accuracy and precision needed for regulatory review, several measurements of groundwater ages from a given geographic region and over various depths could provide a spatial view of groundwater transport rates under associated hydrologic conditions. In general, mean groundwater transport rates would be implicit in this approach, but any temporal variation in these rates, due to variable groundwater flow conditions, would require time-series measurements. Given this premise, the District contracted with the Lawrence Livermore National Laboratory (LLNL) to directly measure groundwater ages using isotope hydrology methods. In particular, LLNL was tasked to define with isotopic analysis the flow paths, flow rates, and mixing of recharge water with groundwater in the Forebay region. The goal of this research was to 1) improve the understanding of the Forebay area hydrogeology and the influence of existing recharge operations on production wells, 2) identify the amount and origin of recharge water in wells for the development of a water quality monitoring program to assess water quality changes as a function of subsurface residence time and proximity to recharge, 3) provide quantitative information in which to evaluate a proposed recycled water recharge project in light of the proposed regulatory criteria, including impacts on the basin and production wells, and 4) use this information to recommend future monitoring approaches.

The research was completed in two phases. The first phase measured the environmental abundance of oxygen-18 $\left(\delta^{18} \mathrm{O}\right)$, deuterium $(\delta \mathrm{D})$, radiocarbon $\left({ }^{14} \mathrm{C}\right)$, 
carbon-13 $\left({ }^{13} \mathrm{C}\right)$, tritium $\left({ }^{3} \mathrm{H}\right)$, helium-3 $\left({ }^{3} \mathrm{He}\right)$ resulting from ${ }^{3} \mathrm{H}$ decay, and dissolved noble gases in groundwater. The intent was to define 1) the general recharge sources and recharge characteristics (recharge temperature, dissolved air, etc.) of groundwater samples using the $\delta^{18} \mathrm{O}, \delta \mathrm{D}$, and dissolved noble gas concentrations, and 2) age date the groundwater within plus or minus one year using ${ }^{3} \mathrm{H}$ and ${ }^{3} \mathrm{He}$ measurements. The goal of the first phase was to better elucidate details of groundwater flow in the Forebay that were not obtainable using existing hydrogeologic and hydrologic data.

The second phase measured abundances of artificial tracers intentionally introduced into recharge water in surface spreading basins and in the SAR channel. The goals here were 1) to quantify groundwater ages (i.e. travel times) from zero to one year, and 2) to quantify the amount of mixing between recharge water and groundwater during downgradient transport. In particular, isotopically enriched xenon gases were used as artificial tracers in the spreading basins. For each of these experiments Colorado River water was labeled with the xenon in order to also utilize $\delta^{18} \mathrm{O}$ measurements as an additional tracer, since the Colorado River $\delta^{18} \mathrm{O}$ value is much lower than SAR water (e.g. Williams, 1997). For tracing groundwater recharged directly from the Santa Ana River, sulfur-hexafluoride $\left(\mathrm{SF}_{6}\right)$ was used as an artificial tracer. This report illustrates all results from the first phase of research and include data from the artificial tracer studies measured until October 2000, or two years after the last recharge experiment commenced.

\section{Hydrogeologic Setting}

The Orange County groundwater basin encompasses approximately $300 \mathrm{mi}^{2}$ stretching from the Chino Hills and Santa Ana Mountains on the north to the Pacific Ocean on the south (Fig. 1a). The basin is connected to the larger Los Angeles basin to the northwest, but traditionally hydrologic boundaries have been placed at the county line for convenience. The Orange County basin was formed from uplift, faulting, and folding of Late Cretaceous and younger sediments beginning in Oligocene time, resulting in a synclinal trough of deposits comprising inter-fingering marine and freshwater sediments spanning Miocene to Holocene time (Fig. 1b).

The Newport-Inglewood fault system over time has created uplifted mesas along the Pacific Ocean coast, which impedes groundwater discharge to the ocean, forming a nearclosed basin condition and a naturally artesian area in the southern basin known as the pressure area. In the northern basin, runoff from the $2670 \mathrm{mi}^{2}$ watershed of the Santa Ana River historically recharged the basin along a coarse-grained sedimentary cone known as the Forebay.

The groundwater basin is defined by the depth of freshwater occurrence, which in the central portion of the basin reaches nearly 4000 feet below the surface (Herndon, 1992). Although freshwater occurs in Miocene-age sediments at depth, the primary freshwater bearing strata are likely Pliocene or younger. In the pressure area, sedimentary deposits are inter-layered coarse-grained sand and gravel and fine-grained silt and clay forming confined aquifer zones. In the Forebay area, consolidated Miocene to Pliocene-age sediments form a basement rock with low groundwater storage, which is overlain by greater than 1000 feet of unconsolidated gravels, sands, and silts that are laterally discontinuous. As a result, groundwater is usually unconfined to semi-confined in the Forebay. 
As a result of near-closed basin conditions and low annual rainfall (14.1 inches per year in Yorba Linda) locally in the basin, early agricultural development, that used groundwater for irrigation, overdrafted the basin nearly 700,000 acre-ft by the 1950s. In addition, with the subsequent urban development of the basin to its current level of approximately two million inhabitants, groundwater became the primary source of drinking water. Today nearly $70 \%$ of the domestic consumption is met by production from the groundwater basin. This pumping sustains a below sea level groundwater table configuration in the pressure area (Fig. 1a). Basin-wide groundwater levels are approximately stabilized by artificial recharge practices conducted along the coast and in the Forebay. Along the coast, historical overdraft caused seawater intrusion through the Talbert Gap (Fig. 1a). The intrusion was reversed by operation of a seawater injection barrier and a 15 million gallons per day water recycling plant that injects highly treated waste water into the barrier to maintain water levels.

In the Forebay, recharge to the basin is maintained by infiltration of the Santa Ana River (Fig. 2a). Before, 1950, the river channel itself was the main recharge mechanism to the basin. In the early 1960s increased water demands and insufficient recharge capacity required converting abandoned gravel mine pits, adjacent to and north of the channel, to artificial recharge basins. SAR flow is diverted from the main channel at the Imperial Head gates and recharged through these basins. The current configuration of SAR diversion directs water from the Imperial Headgates, which has an inflatable dam to pool water, to Conrock Basin. From Conrock, the water flows by gravity to Warner Basin. The water can flow from Warner over to Anaheim Lake, which gravity feeds water over to Miller and Kraemer Basin. Anaheim Lake and Kraemer Basin are drained semi-annually and silts are removed from the bottom to restore percolation capacity. Warner Basin, on the other hand, was historically only drained once every 3-5 years. Since 1999, Warner Basin has been drained approximately once a year for cleaning. Altogether Warner Basin, Anaheim Lake, Miller Basin, and Kraemer Basin annually recharge approximately 100,000 acre-ft, with the majority occurring in Kraemer Basin and Anaheim Lake. The SAR channel recharges approximately 70,000 acre-ft per year. The remainder of recharge in the Forebay occurs in the basins adjacent to the SAR west of Warner Basin and in the Santiago Pits.

As early as 1948, Colorado River water, purchased through the Metropolitan Water District of Southern California, was diverted to the Forebay for recharge purposes (Fig. 2a). Between 1948 and 1962, approximately 970,000 acre-ft of Colorado River water was diverted directly to the SAR channel for recharge. Afterward Colorado River water was diverted to Anaheim Lake where as of 1996 an additional 1.8 million acre-ft were recharged. 

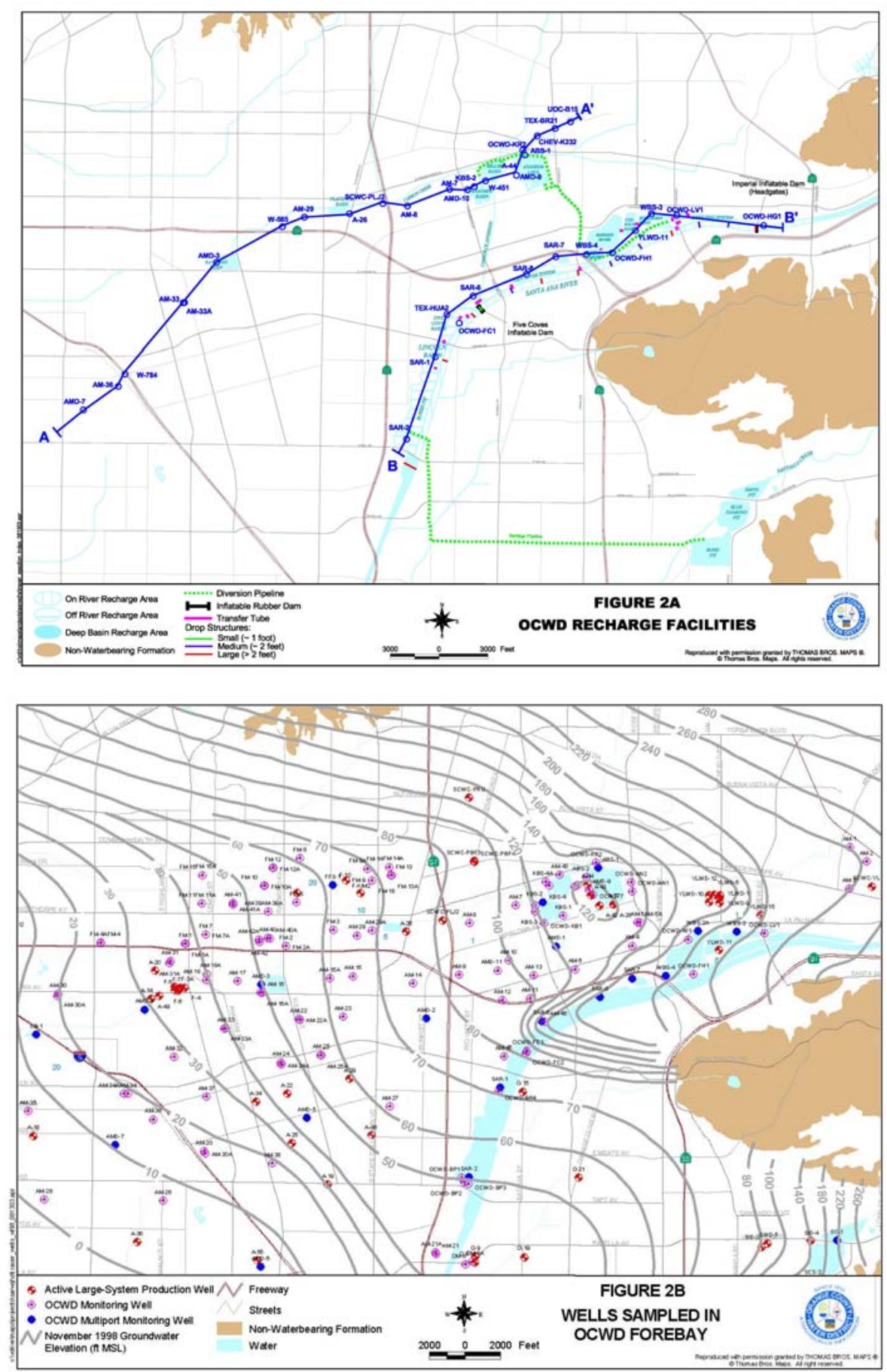

Figure 2. a) Map view of Forebay region with recharge facilities. b) Wells sampled in age-dating and tracer studies, and include water levels from November 1998. 

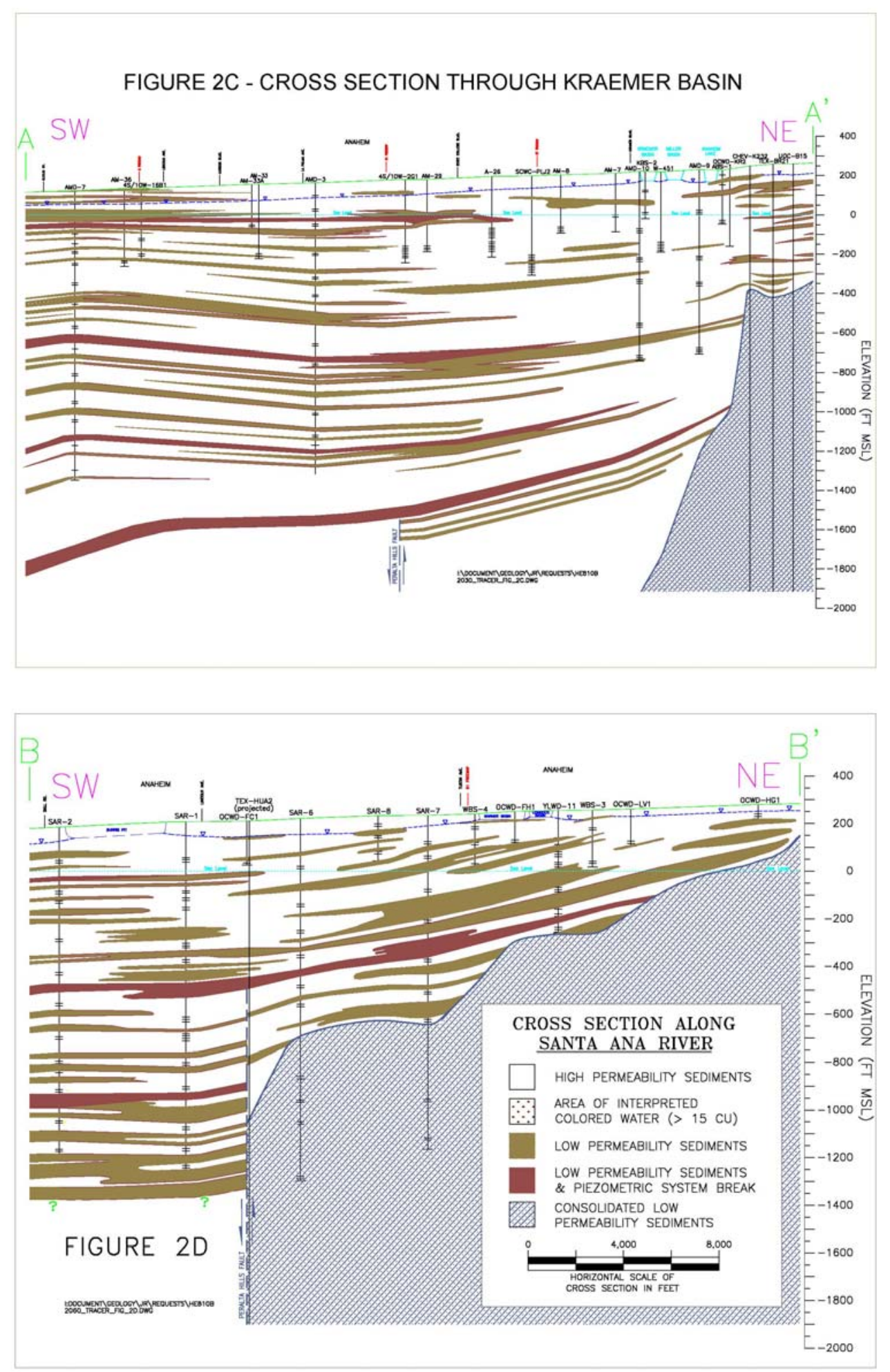

Figure 2. c) Map view of the Forebay region with monitoring and production wells sampled during this study. d) Cross-section view of Forebay with selected monitoring and production wells projected along lines A-A' and B-B'. 
An additional recharge source comes from the State Water Project completed in the early 1970s. This source is periodically released to the SAR channel upstream of the Imperial Headgates and recharges the basin both through channel infiltration and diversion to the basins. Between 1972 and 1996 a total of approximately 234,000 acre-ft of State Water Project supplies were recharged, with nearly $87 \%$ of this recharged between 1972 and 1980.

Recharge in the basins replenishes groundwater to the aquifers used for domestic consumption. In the central and southern portion of the groundwater basin, the aquifers used for production are separated from shallower aquifers by a relatively extensive low permeability layer occurring around 100 to 300 feet below the surface (Fig. 1b). This layer tapers out in the western part of the Forebay area and inter-fingers with spatially discontinuous high and low permeability layers across the Forebay. Below Anaheim Lake, a low permeability layer occurs at about 400 feet below the surface and is thought to retard vertical transport of Anaheim Lake recharge to deeper depths. A low permeability zone also occurs below and west of Kraemer Basin at a shallower depth and likely directs basin recharge to deeper levels and towards the main aquifer system to the west (Fig. 2b).

Beneath the SAR channel, discontinuous layering makes simple flow paths difficult to deduce. At approximately 500 to 700 feet below the surface, there is a low permeability layer that is laterally extensive and connects to the west with a regionally extensive confining layering separating the upper main and lower main aquifers. The consolidated Miocene to Pliocene-age basement rocks occur below the surface in the eastern Forebay ranging from 200 feet to approximately 850 feet in depth (Fig. 2b). Also just west of monitoring well SAR-6, the Peralta Hills fault offsets basement rocks and apparently impedes westerly groundwater flow in this vicinity (Herndon, 1992).

\section{Research Approach}

Phase One of LLNL's efforts to age date groundwater and trace recharge water began in 1995. Initial work entailed selection of surface water sites and a few monitoring and production wells for reconnaissance sampling. LLNL personnel participated in collecting those samples, while at the same time training District field technicians the necessary sampling techniques. From the samples, LLNL generated a multi-isotope database for groundwater source identification and age-dating. This database consisted of approximately 30 samples measured for $\delta{ }^{18} \mathrm{O}, \delta \mathrm{D},{ }^{13} \mathrm{C}$, and ${ }^{14} \mathrm{C}$ of the dissolved inorganic carbon, ${ }^{3} \mathrm{H},{ }^{3} \mathrm{He},{ }^{4} \mathrm{He}$, and noble gas abundance. Discussion regarding the purpose of each of these measurements and general methods for their interpretation can be found in Appendix 1.

Analysis of the reconnaissance database revealed that $\delta^{18} \mathrm{O},{ }^{3} \mathrm{H},{ }^{3} \mathrm{He}$, and ${ }^{4} \mathrm{He}$ adequately determined the source and age of groundwater throughout the Forebay with a precision approximately plus or minus one year (see Davisson et al., 1996). Therefore, starting in 1996, >100 samples were collected for these measurements over a broad region that included areas to the west and southwest of the recharge basins, such as the City of Fullerton and the City of Anaheim (Fig. 3a). The completion of this database within the subsequent year provided detail on the distribution of groundwater ages and recharge sources from 200 to 1000 feet below the ground surface (see Fig. 3b; Davisson et al., 1999; Yoshiba, 1999). This database provided the necessary detail to support the 
District efforts for basin-wide groundwater modeling and to define specific groundwater flow paths targeted for more detailed study using artificial tracers during Phase Two.

Phase Two work of LLNL's work focused on high resolution age-dating and flow path analysis using controlled recharge experiments and artificial tracers. This work was necessary in order to resolve mixtures of less than one year old groundwater in the Forebay. Even though the ${ }^{3} \mathrm{H}^{-}{ }^{3} \mathrm{He}$ measurements were used to calculate groundwater ages up to approximately 40 years old, uncertainties resulting from these calculations had limited use for addressing regulatory guidelines regarding groundwater residence time less than one year. This was particularly true for groundwater ages calculated between one and two years, whose uncertainty was approximately $100 \%$. In addition, the Forebay aquifers are heterogeneous, which causes relatively high amounts of dispersion during groundwater flow. Dispersion in a single flow path is characterized by a combination of fast-moving flow paths and slow-moving flow paths. $\mathrm{A}{ }^{3} \mathrm{H}-{ }^{3} \mathrm{He}$ age represents a mixture of groundwater with fast and slow flow paths, resulting in a mixed or weighted mean age. This means that some component of a groundwater sample could have an age less than one year even though the calculated age of the bulk sample is greater.

In October of 1996, the first recharge tracer experiment was conducted. This involved the recharge of 6300 acre-ft of Colorado River water exclusively into Anaheim Lake. This was followed by the introduction of $a^{124} \mathrm{Xe}$ isotope tracer into the lake. The xenon tracer had an isotope abundance highly enriched relative to its natural isotope abundance. This imparted a strong, chemically inert signature to the recharging water without compromising potable uses of the water. Furthermore, the distinct $\delta^{18} \mathrm{O}$ abundance of the Colorado River water relative to SAR water provided an easily measured tracer of the recharged water.

Based on the success of the 1996 recharge experiment, in October 1998, approximately 9500 acre-ft of Colorado River water was diverted into both Anaheim Lake and Kraemer Basin for more thorough analysis of groundwater recharge and flow in this part of the Forebay. This was followed by introduction of a ${ }^{129} \mathrm{Xe}$ tracer into Anaheim Lake, and a ${ }^{136} \mathrm{Xe}$ tracer into Kramer basin. These tracers were also isotopically-enriched, and their distinct enrichment character imparted tracer labels to groundwater that uniquely differentiated recharge from the two basins. The $\delta^{18} \mathrm{O}$ abundance also provided an additional tracer signal, but because Colorado River water was recharged in both basins, it was not able to differentiate Anaheim Lake recharge from Kraemer Basin recharge except in cases where hydrologic head differences excluded one source from the other.

In the summer of 1998 , low concentrations of $\mathrm{SF}_{6}$ tracer were added to approximately 3000 acre-ft of Santa Ana River water. Sulfur-hexafluoride is an inert gas that does not occur naturally and can be detected at part per trillion levels. The concentration in SAR water that was recharged was approximately 1000 times above the detection limit, imparting an easily identifiable signature unique to Santa Ana River water recharge. The recharge experiment was conducted during the summer at base flow conditions when control over flow conditions in the SAR was greatest.

The simultaneous introduction of the xenon tracers and $\mathrm{SF}_{6}$ tracer in 1998 provided an opportunity to observe co-mingling of recharge from Santa Ana River with recharge from Anaheim Lake and Kraemer Basin. In order to capture the necessary observational details, an aggressive sampling strategy was developed to sample wells at specific 
frequencies over a two-year period. The frequencies were determined based on the ${ }^{3} \mathrm{H}-$ ${ }^{3} \mathrm{He}$ age data and the results of the 1996 recharge experiment from Anaheim Lake. Sampling frequencies were further modified during the sampling program when necessary based on tracer arrival times and persistence in wells.

The artificial tracer experiments were highly successful in determining fast flow paths in groundwater recharged in the Forebay. It furthermore provided valuable hydrologic data on aquifer permeability and dispersivity. In some cases, the tracer data provided good evidence for groundwater dilution. However, because the amount of incidental groundwater recharge to the Forebay aquifers was small compared to the total groundwater storage, groundwater dispersion in most cases masked the ability to identify dilution with native groundwater.

\section{Analytical Methods}

Details of sampling methodology and analytical procedures are outlined in Appendix 2. All the elemental isotopes that were measured for the District in their natural chemical form either have a high volatility or are sensitive to atmospheric exchange. As a result, samples were collected in air-tight containers, and preparation procedures for analysis were conducted under vacuum conditions. All analyses utilized magnetic sector mass spectrometry instruments.

\section{Field Methods}

The majority of groundwater was sampled from either domestic supply wells with dedicated pumps, monitoring wells with dedicated submersible pumps, or from monitoring wells into which a portable submersible pump was temporarily lowered. At least three well volumes were pumped before sampling. Westbay-type multiport monitoring wells required no purging and groundwater was collected in evacuated stainless steel containers. Noble gas samples were collected from Westbay wells with a modified sampling tool, which is discussed in Appendix 3. Surface water samples were either a grab sample collected from the shore or collected with a peristaltic pump at different depths from a boat in the center of the spreading basins. SAR samples were collected at various points along the river.

Temperature, electrical conductivity (EC), $\mathrm{pH}$, and dissolved oxygen were measured in the field when possible at the time of sampling. Samples were collected for analysis of ${ }^{18} \mathrm{O}$, deuterium, carbon isotopes, and ${ }^{3} \mathrm{H}$ in glass, air-tight sealed bottles. The noble gas and $\mathrm{SF}_{6}$ sampling required a copper tube pinch-clamp assembly that sealed a water sample in a copper tube free of any gas bubbles. Details of the sampling procedures for all well types are provided in Appendix 3.

\section{Results From the Environmental Isotope Characterization and Age-Dating}

\subsection{Age-Dating Results}

Resolving groundwater ages using the ${ }^{3} \mathrm{H}-{ }^{3} \mathrm{He}$ measurements depends to some extent on the amount of ${ }^{3} \mathrm{H}$ originally present in a groundwater at the time of recharge. The ${ }^{3} \mathrm{He}$ measurement and tritiogenic ${ }^{3} \mathrm{He}$ calculation potentially result in high uncertainty. Consequently, the higher the ${ }^{3} \mathrm{H}$ content of a recharging groundwater, the more tritiogenic ${ }^{3} \mathrm{He}$ produced over time and the more easily the tritiogenic ${ }^{3} \mathrm{He}$ content is detected and 
quantified. This translates into higher precision of the calculated tritiogenic ${ }^{3} \mathrm{He}$ and a better resolved age.

\subsection{1 $\left({ }^{3} \mathrm{H}\right)$ Tritium Results}

The ${ }^{3} \mathrm{H}$ concentration was measured in SAR water and associated recharge basins in 1995 during the initial age-dating characterization of Forebay groundwater. At the time the ${ }^{3} \mathrm{H}$ was between 14 and $24 \mathrm{pCi} / \mathrm{L}$ (Davisson et al., 1996). These values were similar to runoff reported approximately 2 years earlier by Williams (1994) for local creek flow from the Santa Ana Mountains $(10-16 \mathrm{pCi} / \mathrm{L})$ and for sewage treatment plant water discharged upstream of Prado Dam (17-24 pCi/L). Note that the ${ }^{3} \mathrm{H}$ of Santa Ana Mountains runoff is slightly lower than the waste water discharge. The latter is derived from water sources originating from a combination of groundwater, upstream Santa Ana River runoff, and State Water Project, whose higher ${ }^{3} \mathrm{H}$ concentrations indicate more inland, higher elevation sources, or slightly older water in the case of groundwater.

Tritium concentrations measured in Forebay groundwater range from less than 1 to $144 \mathrm{pCi} / \mathrm{L}$ (Table 1). Tritium defined a more narrow range from 13 to $43 \mathrm{pCi} / \mathrm{L}$ in groundwater east of Hwy 57 (closer to recharge basins), whereas west of Hwy 57, highest concentrations were observed. This is consistent with older groundwater occurring west of Hwy 57 having higher atmospheric fallout ${ }^{3} \mathrm{H}$ concentrations. In three instances of deep groundwater west of Hwy 57 (AMD-5 level 12, AMD-6 level 10, AMD-7 level 13), groundwater ${ }^{3} \mathrm{H}$ concentrations were essentially non-detect, indicating groundwater $>40$ years old. The highest measured ${ }^{3} \mathrm{H}$ concentration found in deep groundwater west of Hwy 57 was in AMD-7 level 10.

Like most places around the world, ${ }^{3} \mathrm{H}$ fallout from nuclear testing recorded in precipitation of coastal California was highest around 1963. However, no ${ }^{3} \mathrm{H}$ records of precipitation exist for this time period in the SAR watershed. Nevertheless, records of ${ }^{3} \mathrm{H}$ fallout over southern coastal California can be evaluated using ${ }^{3} \mathrm{H}$ measurements of precipitation collected at Santa Maria, along the central California coast (IAEA/WMO, 2001). During this time period, the ${ }^{3} \mathrm{H}$ decreased from approximately $2200 \mathrm{pCi} / \mathrm{L}$ in 1963 to approximately $50 \mathrm{pCi} / \mathrm{L}$ in the 1970 s (IAEA/WMO, 2001). This ${ }^{3} \mathrm{H}$ fallout pattern is similar to that observed world-wide.

It is important to recognize that the Colorado River water imported and recharged into the Forebay was ultimately derived from high elevations of the continental interior in Colorado. Here ${ }^{3} \mathrm{H}$ concentration in precipitation was significantly greater than observed in coastal California. For example, the ${ }^{3} \mathrm{H}$ concentration measured in the Colorado River above Cisco, Utah exceeded $1000 \mathrm{pCi} / \mathrm{L}$ in the late 1960s (Michel, 1992) and was nearly five times greater than Santa Maria precipitation in the same year (Fig. 3). Tritium concentrations measured in Lake Mead, Nevada (impounded Colorado River) varied from 220 to $60 \mathrm{pCi} / \mathrm{L}$ between 1983 and 1991 (Holloway, 1993), which was approximately three times greater concentration in than Santa Maria precipitation. 
Table 1: Preliminary Isotope Data, Ages, and Recharge Temperatures calculated for Groundwater in the Forebay

\begin{tabular}{|c|c|c|c|c|c|c|c|c|c|c|c|}
\hline sample & collection date & $\delta^{18} \mathrm{O}$ & $\delta \mathbf{D}$ & ${ }^{3} \mathrm{H} \mathrm{pCi} / \mathrm{L}$ & ${ }^{3} \mathrm{H}-{ }^{3} \mathrm{He}$ age (yrs) & ${ }^{3} \mathrm{H}$ initial pCi/L & recharge $\mathrm{T}^{\circ} \mathrm{C}$ & excess air $\left(\mathrm{cc} / \mathrm{LH}_{2} \mathrm{O}\right)$ & ${ }^{14} \mathrm{C}$ pmc & $\delta^{13} \mathrm{C}$ per mil & $\mathrm{DIC}\left(\mathrm{mg} / \mathrm{L} \mathrm{HCO}_{3}\right)$ \\
\hline A- 16 & 6/30/1997 & -9.15 & -76.2 & 70.0 & 27.9 & 332.0 & - & - & - & - & - \\
\hline A-22 & $6 / 3 / 1997$ & -7.76 & -62.3 & - & - & - & - & - & - & - & - \\
\hline A-26 & $8 / 29 / 1997$ & -7.82 & - & - & - & - & - & - & - & - & - \\
\hline A-26 & $10 / 22 / 1997$ & -7.85 & - & - & - & - & - & - & - & - & - \\
\hline A-26 & $11 / 24 / 1997$ & -7.96 & - & - & - & - & - & - & - & - & - \\
\hline A-27 & 3/9/1995 & -8.30 & -58 & 21.00 & 0.3 & - & 12 & 0.0155 & 115 & -10.6 & 214 \\
\hline A-27 & 6/19/1995 & -7.10 & -51 & 19.00 & 0.0 & - & 21 & 0.0068 & - & - & 221 \\
\hline A-27 & $8 / 28 / 1995$ & - & - & - & - & - & 25 & 0.0023 & - & - & - \\
\hline A-27 & $9 / 20 / 95$ & -7.03 & - & - & - & - & - & - & - & - & - \\
\hline A-27 & $10 / 19 / 95$ & -6.91 & - & - & - & - & - & - & - & - & - \\
\hline A-27 & $11 / 15 / 95$ & -7.20 & - & - & - & - & - & - & - & - & - \\
\hline A-27 & $12 / 1 / 1995$ & -7.57 & - & - & - & - & - & - & - & - & - \\
\hline A-27 & $1 / 17 / 1996$ & -7.67 & - & - & - & - & - & - & - & - & - \\
\hline A-27 & $2 / 28 / 96$ & -7.47 & - & - & - & - & - & - & - & - & - \\
\hline A-27 & 9/27/1996 & -7.53 & - & - & - & - & - & - & - & - & - \\
\hline A-27 & $10 / 1 / 1996$ & -7.63 & - & - & - & - & 32 & 0.0077 & - & - & - \\
\hline A-27 & 10/7/1996 & -7.56 & - & - & - & - & 30 & 0.0065 & - & - & - \\
\hline A-27 & $10 / 11 / 1996$ & -7.62 & - & - & - & - & - & - & - & - & - \\
\hline A-27 & 10/16/1996 & -9.24 & - & - & - & - & - & - & - & - & - \\
\hline A-27 & $10 / 31 / 1996$ & -10.97 & - & - & - & - & - & - & - & - & - \\
\hline A-27 & $1 / 30 / 1997$ & -7.08 & - & - & - & - & - & - & - & - & - \\
\hline A-27 & $11 / 17 / 1997$ & -8.11 & - & - & - & - & - & - & - & - & - \\
\hline A-27 & $12 / 16 / 1997$ & -7.61 & - & - & - & - & - & - & - & - & - \\
\hline A-27 & 10/8/1998 & -7.14 & - & - & - & - & - & - & - & - & - \\
\hline A-27 & $10 / 15 / 1998$ & -9.75 & - & - & - & - & - & - & - & - & - \\
\hline A-27 & $10 / 25 / 1998$ & -11.21 & - & - & - & - & - & - & - & - & - \\
\hline A-27 & $10 / 30 / 1998$ & -11.49 & - & - & - & - & - & - & - & - & - \\
\hline A-27 & 11/7/1998 & -11.46 & - & - & - & - & - & - & - & - & - \\
\hline A-27 & $11 / 10 / 1998$ & -11.42 & - & - & - & - & - & - & - & - & - \\
\hline A-27 & $11 / 13 / 1998$ & -11.43 & - & - & - & - & - & - & - & - & - \\
\hline A-27 & 11/16/1998 & -11.25 & - & - & - & - & - & - & - & - & - \\
\hline A-27 & $11 / 20 / 1998$ & -11.34 & - & - & - & - & - & - & - & - & - \\
\hline A-27 & $11 / 24 / 1998$ & -11.31 & - & - & - & - & - & - & - & - & - \\
\hline A-27 & $12 / 2 / 1998$ & -10.26 & - & - & - & - & - & - & - & - & - \\
\hline A-27 & $12 / 7 / 1998$ & -8.59 & - & - & - & - & - & - & - & - & - \\
\hline A-27 & $12 / 15 / 1998$ & -7.92 & - & - & - & - & - & - & - & - & - \\
\hline A-27 & $12 / 22 / 1998$ & -7.69 & - & - & - & - & - & - & - & - & - \\
\hline A-27 & $12 / 30 / 1998$ & -7.80 & - & - & - & - & - & - & - & - & - \\
\hline A-28 & $6 / 29 / 1996$ & -7.57 & - & - & - & - & - & - & - & - & - \\
\hline A-28 & 7/17/1996 & -7.70 & - & - & 2.8 & - & 25 & 0.0262 & - & - & - \\
\hline A- 28 & 9/27/1996 & -7.70 & - & - & - & - & 25 & 0.0139 & - & - & - \\
\hline A-28 & $10 / 1 / 1996$ & -7.68 & - & - & - & - & - & - & - & - & - \\
\hline A-28 & $10 / 2 / 1996$ & -7.62 & - & - & - & - & - & - & - & - & - \\
\hline A-28 & $10 / 7 / 1996$ & -7.70 & - & - & - & - & - & - & - & - & - \\
\hline
\end{tabular}


Table 1: Preliminary Isotope Data, Ages, and Recharge Temperatures calculated for Groundwater in the Forebay

\begin{tabular}{|c|c|c|c|c|c|c|c|c|c|c|c|}
\hline sample & collection date & $\delta^{18} \mathrm{O}$ & $\delta \mathbf{D}$ & ${ }^{3} \mathrm{H}$ pCi/L & ${ }^{3} \mathrm{H}-{ }^{3} \mathrm{He}$ age (yrs) & ${ }^{3} \mathrm{H}$ initial pCi/L & recharge $\mathrm{T}^{\circ} \mathrm{C}$ & excess air $\left(\mathrm{cc} / \mathrm{LH}_{2} \mathrm{O}\right)$ & ${ }^{14} \mathrm{C}$ pmc & $\delta^{13} \mathrm{C}$ per mil & DIC (mg/L $\left.\mathrm{HCO}_{3}\right)$ \\
\hline A- 28 & $10 / 11 / 1996$ & -7.60 & - & - & - & - & - & - & - & - & $-x^{-}$ \\
\hline A-28 & $10 / 16 / 1996$ & -7.74 & - & - & - & - & - & - & - & - & - \\
\hline A-28 & $10 / 31 / 1996$ & -8.37 & - & - & - & - & - & - & - & - & - \\
\hline A-28 & $1 / 30 / 1997$ & -8.73 & - & - & - & - & - & - & - & - & - \\
\hline A-28 & 2/18/1997 & -8.48 & - & - & - & - & - & - & - & - & - \\
\hline A-28 & $3 / 6 / 1997$ & -8.24 & - & - & - & - & - & - & - & - & - \\
\hline A-28 & 4/1/1997 & -7.79 & - & - & - & - & - & - & - & - & - \\
\hline A-28 & $4 / 22 / 1997$ & -7.77 & - & - & - & - & - & - & - & - & - \\
\hline A-28 & 9/24/1997 & -8.82 & - & - & - & - & - & - & - & - & - \\
\hline A-28 & 10/21/1997 & -8.53 & - & - & - & - & - & - & - & - & - \\
\hline A-28 & 11/17/1997 & -8.18 & - & - & - & - & - & - & - & - & - \\
\hline A-28 & $12 / 16 / 1997$ & -8.10 & - & - & - & - & - & - & - & - & - \\
\hline A- 28 & $10 / 8 / 1998$ & -7.36 & - & - & - & - & - & - & - & - & - \\
\hline A-28 & $10 / 15 / 1998$ & -7.38 & - & - & - & - & - & - & - & - & - \\
\hline A-28 & $10 / 21 / 1998$ & -8.01 & - & - & - & - & - & - & - & - & - \\
\hline A-28 & $11 / 10 / 1998$ & -8.73 & - & - & - & - & - & - & - & - & - \\
\hline A-28 & $11 / 16 / 1998$ & -9.05 & - & - & - & - & - & - & - & - & - \\
\hline A-28 & $11 / 24 / 1998$ & -9.27 & - & - & - & - & - & - & - & - & - \\
\hline A-28 & $12 / 2 / 1998$ & -9.61 & - & - & - & - & - & - & - & - & - \\
\hline A- 28 & 12/7/1998 & -9.75 & - & - & - & - & - & - & - & - & - \\
\hline A- 28 & $12 / 15 / 1998$ & -9.90 & - & - & - & - & - & - & - & - & - \\
\hline A-28 & $12 / 30 / 1998$ & -9.40 & - & - & - & - & - & - & - & - & - \\
\hline A- 28 & $1 / 5 / 1999$ & -9.19 & - & - & - & - & - & - & - & - & - \\
\hline A-28 & 1/19/1999 & -8.84 & - & - & - & - & - & - & - & - & - \\
\hline A-28 & 1/25/1999 & -8.72 & - & - & - & - & - & - & - & - & - \\
\hline A- 28 & $2 / 1 / 1999$ & -8.58 & - & - & - & - & - & - & - & - & - \\
\hline A-42 & $3 / 9 / 1995$ & -8.20 & -58 & 41.00 & 17.7 & 110.0 & 16 & 0.009 & 103 & -11.3 & 223 \\
\hline A-42 & 6/19/1995 & -8.20 & -53 & - & - & - & 15 & 0.009 & - & -11 & 230 \\
\hline A- 42 & 9/20/1995 & -8.16 & - & - & - & - & - & - & - & - & - \\
\hline A- 42 & $10 / 19 / 1995$ & -8.12 & - & - & - & - & - & - & - & - & - \\
\hline A-42 & $11 / 15 / 1995$ & -8.11 & - & - & - & - & - & - & - & - & - \\
\hline A-42 & $12 / 13 / 1995$ & -8.15 & - & - & - & - & - & - & - & - & - \\
\hline A- 42 & $1 / 17 / 1996$ & -8.15 & - & - & - & - & - & - & - & - & - \\
\hline A-42 & 2/29/1996 & -8.24 & - & - & - & - & - & - & - & - & - \\
\hline A- 42 & $4 / 17 / 1996$ & -8.17 & - & - & - & - & - & - & - & - & - \\
\hline A- 42 & $5 / 15 / 1996$ & -8.10 & - & - & - & - & - & - & - & - & - \\
\hline A-42 & $6 / 19 / 1996$ & -8.09 & - & - & - & - & - & - & - & - & - \\
\hline A-42 & 7/17/1996 & -8.13 & - & - & - & - & - & - & - & - & - \\
\hline A- 42 & $8 / 28 / 1996$ & -8.10 & - & - & - & - & - & - & - & - & - \\
\hline A- 42 & $10 / 7 / 1996$ & - & - & - & - & - & 18 & 0.0087 & - & - & - \\
\hline A-42 & $10 / 31 / 1996$ & -8.11 & - & - & - & - & - & - & - & - & - \\
\hline A- 42 & $12 / 3 / 1996$ & -8.02 & - & - & - & - & - & - & - & - & - \\
\hline A- 42 & $12 / 31 / 1996$ & -8.21 & - & - & - & - & - & - & - & - & - \\
\hline A- 42 & $1 / 30 / 1997$ & -8.19 & - & - & - & - & - & - & - & - & - \\
\hline A- 42 & $2 / 18 / 1997$ & -8.09 & - & - & - & - & - & - & - & - & - \\
\hline A- 42 & $2 / 18 / 1997$ & -8.11 & - & - & - & - & - & - & - & - & - \\
\hline A-42 & 4/2/1997 & -8.12 & - & - & - & - & - & - & - & - & - \\
\hline
\end{tabular}


Table 1: Preliminary Isotope Data, Ages, and Recharge Temperatures calculated for Groundwater in the Forebay

\begin{tabular}{|c|c|c|c|c|c|c|c|c|c|c|c|}
\hline sample & collection date & $\delta^{18} \mathbf{O}$ & $\delta \mathbf{D}$ & ${ }^{3} \mathrm{H} \mathrm{pCi} / \mathrm{L}$ & ${ }^{3} \mathrm{H}-{ }^{3} \mathrm{He}$ age (yrs) & ${ }^{3} \mathrm{H}$ initial pCi/L & recharge $\mathrm{T}^{\circ} \mathrm{C}$ & excess air $\left(\mathrm{cc} / \mathrm{LH}_{2} \mathrm{O}\right)$ & ${ }^{14} \mathrm{C}$ pmc & $\delta^{13} \mathrm{C}$ per mil & DIC (mg/L $\left.\mathrm{HCO}_{3}\right)$ \\
\hline $\mathrm{A}-42$ & $7 / 15 / 1997$ & -8.08 & - & - & - & - & - & - & - & - & - \\
\hline A-42 & $8 / 26 / 1997$ & -8.03 & - & - & - & - & - & - & - & - & - \\
\hline A- 42 & 9/24/1997 & -8.17 & - & - & - & - & - & - & - & - & - \\
\hline A-42 & $10 / 21 / 1997$ & -8.08 & - & - & - & - & - & - & - & - & - \\
\hline A- 42 & 11/17/1997 & -8.06 & - & - & - & - & - & - & - & - & - \\
\hline A- 42 & $12 / 16 / 1997$ & -8.15 & - & - & - & - & - & - & - & - & - \\
\hline A42 1010' & - & -8.11 & - & - & 16.9 & - & - & - & - & - & - \\
\hline A42 650' & - & -8.18 & - & - & 24.0 & - & - & - & - & - & - \\
\hline A42 780' & - & -8.09 & - & - & 21.5 & - & - & - & - & - & - \\
\hline A42 910' & - & -8.35 & - & - & 25.9 & - & - & - & - & - & - \\
\hline A-43 & $6 / 19 / 1995$ & -8.00 & -59 & 35.00 & 11.7 & 67.0 & 16 & 0.0092 & - & - & - \\
\hline A- 43 & 9/20/1995 & -8.01 & - & - & - & - & - & - & - & - & - \\
\hline A- -43 & $10 / 19 / 1995$ & -7.97 & - & - & - & - & - & - & - & - & - \\
\hline A-43 & $11 / 15 / 1995$ & -7.96 & - & - & - & - & - & - & - & - & - \\
\hline A- 43 & $12 / 13 / 1995$ & -8.08 & - & - & - & - & - & - & - & - & - \\
\hline A-43 & $1 / 17 / 1996$ & -8.06 & - & - & - & - & - & - & - & - & - \\
\hline A-43 & 2/29/1996 & -8.05 & - & - & - & - & - & - & - & - & - \\
\hline A- -43 & 4/17/1996 & -7.82 & - & - & - & - & - & - & - & - & - \\
\hline A- 43 & $5 / 15 / 1996$ & -8.00 & - & - & - & - & - & - & - & - & - \\
\hline A-43 & $6 / 19 / 1996$ & -7.99 & - & - & - & - & - & - & - & - & - \\
\hline A-43 & 7/17/1996 & -8.03 & - & - & - & - & - & - & - & - & - \\
\hline A- 43 & $8 / 28 / 1996$ & -7.99 & - & - & - & - & - & - & - & - & - \\
\hline A- -43 & $10 / 7 / 1996$ & - & - & - & - & - & 20 & 0.0083 & - & - & - \\
\hline A-43 & $10 / 31 / 1996$ & -7.91 & - & - & - & - & - & - & - & - & - \\
\hline A- 43 & $12 / 3 / 1996$ & -7.92 & - & - & - & - & - & - & - & - & - \\
\hline A- 43 & $12 / 31 / 1996$ & -7.91 & - & - & - & - & - & - & - & - & - \\
\hline A-43 & 1/30/1997 & -7.95 & - & - & - & - & - & - & - & - & - \\
\hline A-43 & $4 / 22 / 1997$ & -7.97 & - & - & - & - & - & - & - & - & - \\
\hline A- 43 & $8 / 26 / 1997$ & -7.94 & - & - & - & - & - & - & - & - & - \\
\hline A- -43 & 9/24/1997 & -7.98 & - & - & - & - & - & - & - & - & - \\
\hline A- -43 & $10 / 21 / 1997$ & -7.95 & - & - & - & - & - & - & - & - & - \\
\hline A- 43 & $11 / 13 / 1997$ & -7.97 & - & - & - & - & - & - & - & - & - \\
\hline A43 625' & - & -8.11 & - & - & 15.9 & - & - & - & - & - & - \\
\hline A43 720' & - & -7.98 & - & - & 13.3 & - & - & - & - & - & - \\
\hline A-44 & $5 / 23 / 1996$ & -8.26 & - & - & - & - & 19 & 0.0122 & - & - & - \\
\hline A-44 & 7/17/1996 & -7.91 & - & - & 11.0 & - & - & - & - & - & - \\
\hline A-44 & $10 / 7 / 1996$ & - & - & - & - & - & 18 & 0.0138 & - & - & - \\
\hline A-44 & $10 / 11 / 1996$ & -7.82 & - & - & - & - & - & - & - & - & - \\
\hline A-44 & $12 / 3 / 1996$ & -7.88 & - & - & - & - & - & - & - & - & - \\
\hline A-44 & $12 / 31 / 1996$ & -7.99 & - & - & - & - & - & - & - & - & - \\
\hline A-44 & $1 / 30 / 1997$ & -7.71 & - & - & - & - & - & - & - & - & - \\
\hline A- -44 & $2 / 14 / 1997$ & -7.82 & - & - & - & - & - & - & - & - & - \\
\hline A-44 & $4 / 22 / 1997$ & -8.08 & - & - & - & - & - & - & - & - & - \\
\hline A-44 & $5 / 20 / 1997$ & -8.09 & - & - & - & - & - & - & - & - & - \\
\hline A-44 & 6/18/1997 & -8.06 & - & - & - & - & - & - & - & - & - \\
\hline A- 44 & $7 / 15 / 1997$ & -7.95 & - & - & - & - & - & - & - & - & - \\
\hline
\end{tabular}


Table 1: Preliminary Isotope Data, Ages, and Recharge Temperatures calculated for Groundwater in the Forebay

\begin{tabular}{|c|c|c|c|c|c|c|c|c|c|c|c|}
\hline sample & collection date & $\delta^{18} \mathrm{O}$ & $\delta \mathbf{D}$ & ${ }^{3} \mathrm{H} \mathrm{pCi} / \mathrm{L}$ & ${ }^{3} \mathrm{H}-{ }^{3} \mathrm{He}$ age (yrs) & ${ }^{3} \mathrm{H}$ initial pCi/L & recharge $\mathrm{T}^{\circ} \mathrm{C}$ & excess air $\left(\mathrm{cc} / \mathrm{LH}_{2} \mathrm{O}\right)$ & ${ }^{14} \mathrm{C} \mathrm{pmc}$ & $\delta^{13} \mathrm{C}$ per mil & DIC (mg/L $\left.\mathrm{HCO}_{3}\right)$ \\
\hline A-44 & 8/26/1997 & -8.01 & - & - & - & - & - & - & - & - & - \\
\hline A-44 & 9/24/1997 & -8.11 & - & - & - & - & - & - & - & - & - \\
\hline A-44 & 10/21/1997 & -8.20 & - & - & - & - & - & - & - & - & - \\
\hline A-44 & $11 / 13 / 1997$ & -8.33 & - & - & - & - & - & - & - & - & - \\
\hline A-44 & $12 / 16 / 1997$ & -8.32 & - & - & - & - & - & - & - & - & - \\
\hline A44 540' & - & -7.87 & - & - & 13.2 & - & - & - & - & - & - \\
\hline A44 670' & - & -8.03 & - & - & 21.5 & - & - & - & - & - & - \\
\hline A44 840' & - & -8.47 & - & - & 29.2 & - & - & - & - & - & - \\
\hline A44 980' & - & -8.90 & - & - & 30.8 & - & - & - & - & - & - \\
\hline A- 47 & $3 / 8 / 1995$ & -8.50 & -60 & 14.00 & 18.9 & 40.0 & 16 & 0.0037 & 84 & -10.2 & 181 \\
\hline AM-4 & - & - & - & - & 1.4 & - & - & - & - & - & - \\
\hline AM-5 & $4 / 10 / 1998$ & - & - & - & 2.2 & - & - & - & - & - & - \\
\hline AM-5A & $4 / 10 / 1998$ & - & - & - & 0.0 & - & - & - & - & - & - \\
\hline AM-6 & $4 / 24 / 1995$ & -8.10 & -60 & 26.00 & 3.4 & 31.0 & 19 & 0.0154 & 99 & -10.4 & 212 \\
\hline AM-6 & 7/9/1996 & - & - & - & 3.4 & - & 18 & 0.01 & - & - & - \\
\hline AM-6 & $12 / 3 / 1996$ & -7.59 & - & - & - & - & - & - & - & - & - \\
\hline AM-6 & 1/27/1997 & -7.39 & - & - & - & - & - & - & - & - & - \\
\hline AM-6 & 3/31/1997 & -7.53 & - & - & - & - & - & - & - & - & - \\
\hline AM-6 & 4/23/1997 & -7.65 & - & - & - & - & - & - & - & - & - \\
\hline AM-6 & $5 / 21 / 1997$ & -7.53 & - & - & - & - & - & - & - & - & - \\
\hline AM-6 & $6 / 23 / 1997$ & -7.51 & - & - & - & - & - & - & - & - & - \\
\hline AM-6 & 7/16/1997 & -7.56 & - & - & - & - & - & - & - & - & - \\
\hline AM-6 & $8 / 23 / 1997$ & -7.61 & - & - & - & - & - & - & - & - & - \\
\hline AM-6 & 10/2/1997 & -7.59 & - & - & - & - & - & - & - & - & - \\
\hline AM-6 & $10 / 23 / 1997$ & -7.63 & - & - & - & - & - & - & - & - & - \\
\hline AM-6 & 11/17/1997 & -7.53 & - & - & - & - & - & - & - & - & - \\
\hline AM-6 & 12/16/1997 & -7.63 & - & - & - & - & - & - & - & - & - \\
\hline AM-6 & 1/18/1999 & -7.74 & - & - & - & - & - & - & - & - & - \\
\hline AM-7 & $6 / 26 / 1995$ & -7.70 & -58 & 21.00 & 2.3 & 24.0 & 18 & 0.0147 & - & -11.7 & 181 \\
\hline AM-7 & 7/17/1996 & - & - & - & 2.5 & - & 26 & 0.0136 & - & - & - \\
\hline AM-7 & $5 / 28 / 1996$ & -7.37 & - & - & - & - & 19 & 0.0224 & - & - & - \\
\hline AM-7 & $12 / 31 / 1996$ & -7.53 & - & - & - & - & - & - & - & - & - \\
\hline AM-7 & $1 / 27 / 1997$ & -7.59 & - & - & - & - & - & - & - & - & - \\
\hline AM-7 & 2/22/1997 & -7.79 & - & - & - & - & - & - & - & - & - \\
\hline AM-7 & $4 / 23 / 1997$ & -7.26 & - & - & - & - & - & - & - & - & - \\
\hline AM-7 & $5 / 22 / 1997$ & -7.38 & - & - & - & - & - & - & - & - & - \\
\hline AM-7 & $6 / 19 / 1997$ & -7.48 & - & - & - & - & - & - & - & - & - \\
\hline AM-7 & $7 / 16 / 1997$ & -7.68 & - & - & - & - & - & - & - & - & - \\
\hline AM-7 & $8 / 28 / 1997$ & -7.78 & - & - & - & - & - & - & - & - & - \\
\hline AM-7 & $10 / 23 / 1997$ & -7.84 & - & - & - & - & - & - & - & - & - \\
\hline AM-7 & $12 / 17 / 1997$ & -6.89 & - & - & - & - & - & - & - & - & - \\
\hline AM-7 & $12 / 30 / 1998$ & -7.82 & - & - & - & - & - & - & - & - & - \\
\hline
\end{tabular}


Table 1: Preliminary Isotope Data, Ages, and Recharge Temperatures calculated for Groundwater in the Forebay

\begin{tabular}{|c|c|c|c|c|c|c|c|c|c|c|c|}
\hline sample & collection date & $\delta^{18} \mathrm{O}$ & $\delta \mathbf{D}$ & ${ }^{3} \mathrm{H}$ pCi/L & ${ }^{3} \mathrm{H}-{ }^{3} \mathrm{He}$ age (yrs) & ${ }^{3} \mathrm{H}$ initial pCi/L & recharge $\mathrm{T}^{\circ} \mathrm{C}$ & excess air $\left(\mathrm{cc} / \mathrm{LH}_{2} \mathrm{O}\right)$ & ${ }^{14} \mathrm{C} \mathrm{pmc}$ & $\delta^{13} \mathrm{C}$ per mil & DIC (mg/L $\left.\mathrm{HCO}_{3}\right)$ \\
\hline AM-7 & 1/6/1999 & -8.45 & - & - & - & - & - & - & - & - & - \\
\hline AM-7 & 1/12/1999 & -10.71 & - & - & - & - & - & - & - & - & - \\
\hline AM-7 & 1/20/1999 & -10.20 & - & - & - & - & - & - & - & - & - \\
\hline AM-7 & $1 / 29 / 1999$ & -9.36 & - & - & - & - & - & - & - & - & - \\
\hline AM-8 & 7/9/1996 & - & - & - & 2.3 & - & 20 & 0.01 & - & - & - \\
\hline AM-8 & $12 / 18 / 1996$ & -7.55 & - & - & - & - & - & - & - & - & - \\
\hline AM-8 & 2/13/1997 & -8.02 & - & - & - & - & - & - & - & - & - \\
\hline AM-8 & $3 / 31 / 1997$ & -7.71 & - & - & - & - & - & - & - & - & - \\
\hline AM-8 & 4/23/1997 & -7.67 & - & - & - & - & - & - & - & - & - \\
\hline AM-8 & $5 / 29 / 1997$ & -7.50 & - & - & - & - & - & - & - & - & - \\
\hline AM-8 & 6/23/1997 & -7.48 & - & - & - & - & - & - & - & - & - \\
\hline AM-8 & 7/17/1997 & -7.46 & - & - & - & - & - & - & - & - & - \\
\hline AM-8 & 8/23/1997 & -7.41 & - & - & - & - & - & - & - & - & - \\
\hline AM-8 & 10/23/1997 & -7.67 & - & - & - & - & - & - & - & - & - \\
\hline AM-8 & 11/17/1997 & -7.67 & - & - & - & - & - & - & - & - & - \\
\hline AM-8 & $12 / 19 / 1997$ & -7.67 & - & - & - & - & - & - & - & - & - \\
\hline AM-9 & $6 / 22 / 1995$ & -9.50 & -74 & 34.00 & 0.0 & - & 18 & 0.0165 & - & -8.5 & 185 \\
\hline AM-9 & 7/17/1996 & -7.88 & - & - & 1.2 & - & 19 & 0.0174 & - & - & - \\
\hline AM-9 & $12 / 16 / 1996$ & -7.62 & - & - & - & - & - & - & - & - & - \\
\hline AM-9 & $12 / 30 / 1996$ & -7.39 & - & - & - & - & - & - & - & - & - \\
\hline AM-9 & 1/30/1997 & -7.47 & - & - & - & - & - & - & - & - & - \\
\hline AM-9 & 4/21/1997 & -7.60 & - & - & - & - & - & - & - & - & - \\
\hline AM-9 & 5/19/1997 & -7.68 & - & - & - & - & - & - & - & - & - \\
\hline AM-9 & $5 / 19 / 1997$ & -7.68 & - & - & - & - & - & - & - & - & - \\
\hline AM-9 & 6/18/1997 & -7.73 & - & - & - & - & - & - & - & - & - \\
\hline AM-9 & $7 / 14 / 1997$ & -7.59 & - & - & - & - & - & - & - & - & - \\
\hline AM-9 & $8 / 26 / 1997$ & -7.72 & - & - & - & - & - & - & - & - & - \\
\hline AM-9 & 9/22/1997 & -7.68 & - & - & - & - & - & - & - & - & - \\
\hline AM-9 & $10 / 20 / 1997$ & -7.74 & - & - & - & - & - & - & - & - & - \\
\hline AM-9 & 11/13/1997 & -7.70 & - & - & - & - & - & - & - & - & - \\
\hline AM-9 & $12 / 15 / 1997$ & -7.79 & - & - & - & - & - & - & - & - & - \\
\hline AM-9 & $10 / 30 / 1998$ & -7.64 & - & - & - & - & - & - & - & - & - \\
\hline AM-9 & $11 / 24 / 1998$ & -7.65 & - & - & - & - & - & - & - & - & - \\
\hline AM-10 & $4 / 25 / 1995$ & -9.70 & -79 & 43.00 & 0.0 & - & 17 & 0.014 & 97 & -8.5 & 187 \\
\hline AM-10 & $5 / 29 / 1996$ & - & - & - & 0.2 & - & 18 & 0.0168 & - & - & - \\
\hline AM-10 & 7/9/1996 & - & - & - & - & - & 17 & 0.02 & - & - & - \\
\hline AM-10 & $12 / 5 / 1996$ & -7.49 & - & - & - & - & - & - & - & - & - \\
\hline AM-10 & $1 / 27 / 1997$ & -7.42 & - & - & - & - & - & - & - & - & - \\
\hline AM-10 & $4 / 22 / 1997$ & -7.60 & - & - & - & - & - & - & - & - & - \\
\hline AM-10 & $5 / 21 / 1997$ & -7.56 & - & - & - & - & - & - & - & - & - \\
\hline AM-10 & $6 / 18 / 1997$ & -7.64 & - & - & - & - & - & - & - & - & - \\
\hline AM-10 & $7 / 15 / 1997$ & -7.64 & - & - & - & - & - & - & - & - & - \\
\hline AM-10 & 8/26/1997 & -7.69 & - & - & - & - & - & - & - & - & - \\
\hline AM-10 & 9/22/1997 & -7.76 & - & - & - & - & - & - & - & - & - \\
\hline AM-10 & 10/20/1997 & -7.83 & - & - & - & - & - & - & - & - & - \\
\hline
\end{tabular}


Table 1: Preliminary Isotope Data, Ages, and Recharge Temperatures calculated for Groundwater in the Forebay

\begin{tabular}{|c|c|c|c|c|c|c|c|c|c|c|c|}
\hline sample & collection date & $\delta^{18} \mathrm{O}$ & $\delta \mathbf{D}$ & ${ }^{3} \mathrm{H}$ pCi/L & ${ }^{3} \mathrm{H}-{ }^{3} \mathrm{He}$ age (vrs) & ${ }^{3} \mathrm{H}$ initial pCi/L & recharge $\mathrm{T}^{\circ} \mathrm{C}$ & excess air $\left(\mathrm{cc} / \mathrm{LH}_{2} \mathrm{O}\right)$ & ${ }^{14} \mathrm{C}$ pmc & $\delta^{13} \mathrm{C}$ per mil & DIC (mg/L $\left.\mathrm{HCO}_{3}\right)$ \\
\hline AM-10 & 11/13/1997 & -7.81 & - & - & - & - & - & - & - & - & - \\
\hline AM-10 & $12 / 15 / 1997$ & -7.86 & - & - & - & - & - & - & - & - & - \\
\hline AM-10 & $10 / 30 / 1998$ & -7.34 & - & - & - & - & - & - & - & - & - \\
\hline AM-10 & $11 / 10 / 1998$ & -5.87 & - & - & - & - & - & - & - & - & - \\
\hline AM-10 & $11 / 25 / 1998$ & -7.38 & - & - & - & - & - & - & - & - & - \\
\hline AM-10 & $12 / 8 / 1998$ & -7.42 & - & - & - & - & - & - & - & - & - \\
\hline AM-10 & 1/5/1999 & -7.43 & - & - & - & - & - & - & - & - & - \\
\hline AM-10 & $1 / 20 / 1999$ & -7.48 & - & - & - & - & - & - & - & - & - \\
\hline AM-11 & - & - & - & - & 1.3 & - & - & - & - & - & - \\
\hline AM-12 & - & - & - & - & 0.0 & - & - & - & - & - & - \\
\hline AM-13 & $7 / 8 / 1996$ & - & - & - & 3.1 & - & 17 & 0.0116 & - & - & - \\
\hline AM-13 & $5 / 2 / 1997$ & -9.20 & - & - & - & - & - & - & - & - & - \\
\hline AM-13 & $12 / 10 / 1998$ & -7.70 & - & - & - & - & - & - & - & - & - \\
\hline AM-13 & $12 / 22 / 1998$ & -7.71 & - & - & - & - & - & - & - & - & - \\
\hline AM-13 & 1/18/1999 & -7.85 & - & - & - & - & - & - & - & - & - \\
\hline AM-14 & 7/9/1996 & - & - & - & 1.9 & - & 25 & 0.0753 & - & - & - \\
\hline AM-14 & $1 / 27 / 1997$ & -7.74 & - & - & - & - & - & - & - & - & - \\
\hline AM-14 & 2/21/1997 & -7.77 & - & - & - & - & - & - & - & - & - \\
\hline AM-14 & $4 / 30 / 1997$ & -7.76 & - & - & - & - & - & - & - & - & - \\
\hline AM-14 & $6 / 27 / 1997$ & -7.73 & -55 & - & 1.9 & - & - & - & - & - & - \\
\hline AM-14 & $7 / 16 / 1997$ & -7.56 & - & - & - & - & - & - & - & - & - \\
\hline AM-14 & 8/27/1997 & -7.68 & - & - & - & - & - & - & - & - & - \\
\hline AM-14 & $10 / 4 / 1997$ & -7.64 & - & - & - & - & - & - & - & - & - \\
\hline AM-14 & $10 / 22 / 1997$ & -7.70 & - & - & - & - & - & - & - & - & - \\
\hline AM-14 & $11 / 17 / 1997$ & -7.68 & - & - & - & - & - & - & - & - & - \\
\hline AM-14 & $12 / 19 / 1997$ & -7.65 & - & - & - & - & - & - & - & - & - \\
\hline AM-14 & $12 / 22 / 1998$ & -8.08 & - & - & - & - & - & - & - & - & - \\
\hline AM-15 & 6/3/1997 & -9.17 & -72 & - & 4.5 & - & - & - & - & - & - \\
\hline AM-17 & - & - & - & - & 23.3 & - & - & - & - & - & - \\
\hline AM-18 & - & - & - & - & 21.2 & - & - & - & - & - & - \\
\hline AM-30 & - & - & - & - & 23.8 & - & - & - & - & - & - \\
\hline AM-33 & 6/27/1997 & -9.55 & -76 & - & 6.8 & - & - & - & - & - & - \\
\hline AM-35 & - & - & - & - & 23.3 & - & - & - & - & - & - \\
\hline AM-44 & $10 / 11 / 1996$ & -7.72 & - & - & - & - & - & - & - & - & - \\
\hline AM-44 & $10 / 16 / 1996$ & -7.71 & - & - & - & - & - & - & - & - & - \\
\hline AM-44 & $10 / 31 / 1996$ & -7.75 & - & - & - & - & - & - & - & - & - \\
\hline AM-44 & $12 / 30 / 1996$ & -8.07 & - & - & - & - & - & - & - & - & - \\
\hline
\end{tabular}


Table 1: Preliminary Isotope Data, Ages, and Recharge Temperatures calculated for Groundwater in the Forebay

\begin{tabular}{|c|c|c|c|c|c|c|c|c|c|c|c|}
\hline sample & collection date & $\delta^{18} \mathrm{O}$ & $\delta \mathbf{D}$ & ${ }^{3} \mathrm{H}$ pCi/L & ${ }^{3} \mathrm{H}-{ }^{3} \mathrm{He}$ age (yrs) & ${ }^{3} \mathrm{H}$ initial pCi/L & recharge $\mathbf{T}^{\circ} \mathrm{C}$ & excess air $\left(\mathrm{cc} / \mathrm{LH}_{2} \mathrm{O}\right)$ & ${ }^{14} \mathrm{C}$ pmc & $\delta^{13} \mathrm{C}$ per mil & DIC (mg/L $\left.\mathrm{HCO}_{3}\right)$ \\
\hline AM-44 & 1/27/1997 & -10.42 & - & - & - & - & - & - & - & - & - \\
\hline AM-44 & 2/21/1997 & -10.55 & - & - & - & - & - & - & - & - & - \\
\hline AM-44 & $3 / 31 / 1997$ & -8.96 & - & - & - & - & - & - & - & - & - \\
\hline AM-44 & $4 / 24 / 1997$ & -8.33 & - & - & - & - & - & - & - & - & - \\
\hline AM-44 & $5 / 22 / 1997$ & -7.51 & - & - & - & - & - & - & - & - & - \\
\hline AM-44 & 9/26/1997 & -9.64 & - & - & - & - & - & - & - & - & - \\
\hline AM-44 & $10 / 22 / 1997$ & -9.36 & - & - & - & - & - & - & - & - & - \\
\hline AM-44 & 11/14/1997 & -8.78 & - & - & - & - & - & - & - & - & - \\
\hline AM-44 & $12 / 17 / 1997$ & -7.99 & - & - & - & - & - & - & - & - & - \\
\hline AMD-1/1 & $12 / 30 / 1996$ & -7.76 & - & - & - & - & - & - & - & - & - \\
\hline AMD-1/1 & 4/24/1997 & -7.59 & - & - & - & - & - & - & - & - & - \\
\hline AMD-1/1 & $5 / 22 / 1997$ & -7.71 & - & - & - & - & - & - & - & - & - \\
\hline AMD-1/1 & $6 / 20 / 1997$ & -7.75 & - & - & - & - & - & - & - & - & - \\
\hline AMD-1/1 & 8/22/1997 & -7.81 & - & - & - & - & - & - & - & - & - \\
\hline AMD-1/1 & 9/22/1997 & -7.87 & - & - & - & - & - & - & - & - & - \\
\hline AMD-1/1 & 10/24/1997 & -7.87 & - & - & - & - & - & - & - & - & - \\
\hline AMD-1/1 & 11/19/1997 & -7.84 & - & - & - & - & - & - & - & - & - \\
\hline AMD-1/1 & 12/19/1997 & -8.22 & - & - & - & - & - & - & - & - & - \\
\hline AMD-1/1 & $12 / 22 / 1998$ & -7.77 & - & - & - & - & - & - & - & - & - \\
\hline AMD-1/1 & 2/1/1999 & -7.75 & - & - & - & - & - & - & - & - & - \\
\hline AMD-1/2 & $11 / 17 / 1995$ & -8.33 & - & - & - & - & - & - & - & - & - \\
\hline AMD-1/2 & 4/24/1997 & -7.98 & - & - & - & - & - & - & - & - & - \\
\hline AMD-1/2 & $5 / 22 / 1997$ & -7.80 & - & - & - & - & - & - & - & - & - \\
\hline AMD-1/2 & 6/20/1997 & -7.79 & - & - & - & - & - & - & - & - & - \\
\hline AMD-1/2 & $7 / 18 / 1997$ & -7.79 & - & - & - & - & - & - & - & - & - \\
\hline AMD-1/2 & $8 / 22 / 1997$ & -7.88 & - & - & - & - & - & - & - & - & - \\
\hline AMD-1/2 & 9/22/1997 & -7.86 & - & - & - & - & - & - & - & - & - \\
\hline AMD-1/2 & $10 / 24 / 1997$ & -8.17 & - & - & - & - & - & - & - & - & - \\
\hline AMD-1/2 & 11/19/1997 & -8.21 & - & - & - & - & - & - & - & - & - \\
\hline AMD-1/2 & 12/19/1997 & -8.34 & - & - & - & - & - & - & - & - & - \\
\hline AMD-1/2 & 12/9/1998 & -7.64 & - & - & - & - & - & - & - & - & - \\
\hline AMD-1/2 & $12 / 22 / 1998$ & -7.67 & - & - & - & - & - & - & - & - & - \\
\hline AMD-1/2 & 1/6/1999 & -7.71 & - & - & - & - & - & - & - & - & - \\
\hline AMD-1/2 & $1 / 20 / 1999$ & -7.68 & - & - & - & - & - & - & - & - & - \\
\hline AMD-1/2 & 2/1/1999 & -6.10 & - & - & - & - & - & - & - & - & - \\
\hline AMD-1/3 & 11/17/1995 & -8.21 & - & - & - & - & - & - & - & - & - \\
\hline AMD-1/3 & 12/30/1996 & -7.50 & - & - & - & - & - & - & - & - & - \\
\hline AMD-1/3 & 4/24/1997 & -7.64 & - & - & - & - & - & - & - & - & - \\
\hline AMD-1/3 & $5 / 22 / 1997$ & -7.85 & - & - & - & - & - & - & - & - & - \\
\hline AMD-1/3 & 6/20/1997 & -8.00 & - & - & - & - & - & - & - & - & - \\
\hline AMD-1/3 & $7 / 18 / 1997$ & -7.90 & - & - & - & - & - & - & - & - & - \\
\hline AMD-1/3 & 8/22/1997 & -8.09 & - & - & - & - & - & - & - & - & - \\
\hline AMD-1/3 & 9/22/1997 & -8.18 & - & - & - & - & - & - & - & - & - \\
\hline AMD-1/3 & $10 / 24 / 1997$ & -8.27 & - & - & - & - & - & - & - & - & - \\
\hline
\end{tabular}


Table 1: Preliminary Isotope Data, Ages, and Recharge Temperatures calculated for Groundwater in the Forebay

\begin{tabular}{|c|c|c|c|c|c|c|c|c|c|c|c|}
\hline sample & collection date & $\delta^{18} \mathrm{O}$ & $\delta \mathbf{D}$ & ${ }^{3} \mathrm{H} \mathrm{pCi} / \mathrm{L}$ & ${ }^{3} \mathrm{H}-{ }^{3} \mathrm{He}$ age (yrs) & ${ }^{3} \mathrm{H}$ initial pCi/L & recharge $\mathrm{T}^{\circ} \mathrm{C}$ & excess air $\left(\mathrm{cc} / \mathrm{LH}_{2} \mathrm{O}\right)$ & ${ }^{14} \mathrm{C} \mathrm{pmc}$ & $\delta^{13} \mathrm{C}$ per mil & $\mathrm{DIC}\left(\mathrm{mg} / \mathrm{L} \mathrm{HCO}_{3}\right)$ \\
\hline AMD-1/3 & $12 / 19 / 1997$ & -8.12 & - & - & - & - & - & - & - & - & - \\
\hline AMD-1/3 & $12 / 9 / 1998$ & -7.69 & - & - & - & - & - & - & - & - & - \\
\hline AMD-1/3 & $12 / 22 / 1998$ & -7.79 & - & - & - & - & - & - & - & - & - \\
\hline AMD-1/3 & $1 / 6 / 1999$ & -7.72 & - & - & - & - & - & - & - & - & - \\
\hline AMD-1/3 & 1/20/1999 & -7.68 & - & - & - & - & - & - & - & - & - \\
\hline AMD-1/3 & 2/1/1999 & -7.70 & - & - & - & - & - & - & - & - & - \\
\hline AMD-1/4 & $11 / 17 / 1995$ & -8.40 & - & - & - & - & - & - & - & - & - \\
\hline AMD-1/4 & Jan-96 & -8.46 & - & - & - & - & - & - & - & - & - \\
\hline AMD-1/4 & $5 / 22 / 1996$ & - & - & - & 1.6 & - & - & - & - & - & - \\
\hline AMD-1/4 & $5 / 22 / 1996$ & -8.46 & - & - & 1.8 & - & - & - & - & - & - \\
\hline AMD- $1 / 4$ & $5 / 22 / 1996$ & - & - & - & 2.1 & - & - & - & - & - & - \\
\hline AMD-1/4 & $11 / 20 / 1996$ & -8.24 & - & - & - & - & - & - & - & - & - \\
\hline AMD-1/4 & 2/20/1997 & -8.04 & - & - & - & - & - & - & - & - & - \\
\hline AMD-1/4 & 4/1/1997 & -8.00 & - & - & - & - & - & - & - & - & - \\
\hline AMD-1/4 & $4 / 24 / 1997$ & -8.61 & - & - & - & - & - & - & - & - & - \\
\hline AMD-1/4 & $5 / 22 / 1997$ & -8.50 & - & - & - & - & - & - & - & - & - \\
\hline AMD- $1 / 4$ & $6 / 20 / 1997$ & -8.50 & - & - & - & - & - & - & - & - & - \\
\hline AMD-1/4 & $7 / 18 / 1997$ & -8.47 & - & - & - & - & - & - & - & - & - \\
\hline AMD-1/4 & $8 / 22 / 1997$ & -8.35 & - & - & - & - & - & - & - & - & - \\
\hline AMD-1/4 & 9/22/1997 & -8.22 & - & - & - & - & - & - & - & - & - \\
\hline AMD-1/4 & $10 / 24 / 1997$ & -8.27 & - & - & - & - & - & - & - & - & - \\
\hline AMD-1/4 & $11 / 19 / 1997$ & -8.47 & - & - & - & - & - & - & - & - & - \\
\hline AMD-1/4 & $12 / 19 / 1997$ & -8.51 & - & - & - & - & - & - & - & - & - \\
\hline AMD-1/4 & 12/9/1998 & -8.18 & - & - & - & - & - & - & - & - & - \\
\hline AMD-1/4 & $12 / 22 / 1998$ & -8.16 & - & - & - & - & - & - & - & - & - \\
\hline AMD-1/4 & 1/6/1999 & -8.19 & - & - & - & - & - & - & - & - & - \\
\hline AMD-1/4 & 1/20/1999 & -8.15 & - & - & - & - & - & - & - & - & - \\
\hline AMD-1/4 & 2/1/1999 & -8.09 & - & - & - & - & - & - & - & - & - \\
\hline AMD-1/5 & $11 / 17 / 1995$ & -8.01 & - & - & - & - & - & - & - & - & - \\
\hline AMD- $1 / 5$ & Jan-96 & -8.00 & - & - & - & - & - & - & - & - & - \\
\hline AMD-1/5 & 2/1/1999 & -7.94 & - & - & - & - & - & - & - & - & - \\
\hline AMD-1/6 & $11 / 17 / 1995$ & -8.07 & - & - & - & - & - & - & - & - & - \\
\hline AMD- $1 / 6$ & Jan-96 & -8.01 & - & - & - & - & - & - & - & - & - \\
\hline AMD-1/7 & $11 / 17 / 1995$ & -8.26 & - & - & - & - & - & - & - & - & - \\
\hline AMD-1/7 & Jan-96 & -8.12 & - & - & - & - & - & - & - & - & - \\
\hline AMD-1/7 & $5 / 22 / 1996$ & -8.12 & - & - & 5.9 & - & - & - & - & - & - \\
\hline AMD-1/7 & $11 / 20 / 1996$ & -8.13 & - & - & - & - & - & - & - & - & - \\
\hline AMD-1/8 & $11 / 17 / 1995$ & -8.03 & - & - & - & - & - & - & - & - & - \\
\hline AMD-1/8 & Jan-96 & -8.05 & - & - & - & - & - & - & - & - & - \\
\hline AMD-1/8 & $5 / 22 / 1996$ & -8.05 & - & - & 10.3 & - & 8 & 0.0181 & - & - & - \\
\hline AMD-1/8 & $11 / 20 / 1996$ & -8.08 & - & - & - & - & - & - & - & - & - \\
\hline AMD-1/9 & $11 / 17 / 1995$ & -7.87 & - & - & - & - & - & - & - & - & - \\
\hline
\end{tabular}


Table 1: Preliminary Isotope Data, Ages, and Recharge Temperatures calculated for Groundwater in the Forebay

\begin{tabular}{|c|c|c|c|c|c|c|c|c|c|c|c|}
\hline sample & collection date & $\delta^{18} \mathbf{O}$ & $\delta \mathbf{D}$ & ${ }^{3} \mathrm{H} \mathrm{pCi} / \mathrm{L}$ & ${ }^{3} \mathrm{H}-{ }^{3} \mathrm{He}$ age (yrs) & ${ }^{3} \mathrm{H}$ initial pCi/L & recharge $\mathbf{T}^{\circ} \mathrm{C}$ & excess air $\left(\mathrm{cc}^{-} \mathrm{LH}_{2} \mathrm{O}\right)$ & ${ }^{14} \mathrm{C}$ pmc & $\delta^{13} \mathrm{C}$ per mil & DIC (mg/L $\left.\mathrm{HCO}_{3}\right)$ \\
\hline AMD-1/9 & Jan-96 & -7.89 & - & - & - & - & - & - & - & - & - \\
\hline AMD-1/9 & $5 / 22 / 1996$ & - & - & - & 9.0 & - & - & - & - & - & - \\
\hline AMD-1/9 & $11 / 20 / 1996$ & -7.81 & - & - & - & - & - & - & - & - & - \\
\hline AMD- $1 / 10$ & $11 / 17 / 1995$ & -8.07 & - & - & - & - & - & - & - & - & - \\
\hline AMD- $1 / 10$ & Jan-96 & -8.05 & - & - & - & - & - & - & - & - & - \\
\hline AMD-2/1 & $11 / 10 / 1995$ & -8.15 & - & - & - & - & - & - & - & - & - \\
\hline AMD-2/1 & Jan-96 & -7.73 & - & - & - & - & - & - & - & - & - \\
\hline AMD-2/1 & $5 / 21 / 1996$ & - & - & - & 4.9 & - & - & - & - & - & - \\
\hline AMD-2/2 & $11 / 10 / 1995$ & -8.09 & - & - & - & - & - & - & - & - & - \\
\hline AMD-2/2 & Jan-96 & -8.04 & - & - & - & - & - & - & - & - & - \\
\hline AMD-2/2 & $12 / 15 / 1997$ & -7.97 & - & - & - & - & - & - & - & - & - \\
\hline AMD- $2 / 3$ & $11 / 10 / 1995$ & -8.08 & - & - & - & - & - & - & - & - & - \\
\hline AMD-2/3 & Jan-96 & -8.04 & - & - & - & - & - & - & - & - & - \\
\hline AMD-2/4 & $11 / 10 / 1995$ & -7.95 & - & - & - & - & - & - & - & - & - \\
\hline AMD-2/4 & Jan-96 & -7.94 & - & - & 8.0 & - & - & - & - & - & - \\
\hline AMD-2/4 & $5 / 21 / 1996$ & - & - & - & 6.7 & - & - & - & - & - & - \\
\hline AMD-2/4 & $5 / 21 / 1996$ & - & - & - & 9.0 & - & - & - & - & - & - \\
\hline AMD-2/5 & $11 / 10 / 1995$ & -6.93 & - & - & - & - & - & - & - & - & - \\
\hline AMD-2/5 & Jan-96 & -7.39 & - & - & - & - & - & - & - & - & - \\
\hline AMD-2/5 & $5 / 21 / 1996$ & - & - & - & 15.9 & - & 17 & 0.0103 & - & - & - \\
\hline AMD-2/6 & $11 / 10 / 1995$ & -7.83 & - & - & - & - & - & - & - & - & - \\
\hline AMD-2/6 & Jan-96 & -7.93 & - & - & - & - & - & - & - & - & - \\
\hline AMD-2/7 & 11/10/1995 & -8.01 & - & - & - & - & - & - & - & - & - \\
\hline AMD-2/7 & Jan-96 & -7.97 & - & - & - & - & - & - & - & - & - \\
\hline AMD-2/7 & $5 / 21 / 1996$ & -7.97 & - & - & 30.6 & - & 10 & 0.0164 & - & - & - \\
\hline AMD-2/8 & $11 / 10 / 1995$ & -7.40 & - & - & - & - & - & - & - & - & - \\
\hline AMD-2/8 & Jan-96 & -7.39 & - & - & - & - & - & - & - & - & - \\
\hline AMD-2/8 & $5 / 21 / 1996$ & -7.39 & - & - & 23.9 & - & - & - & - & - & - \\
\hline AMD-2/9 & $11 / 10 / 1995$ & -7.31 & - & - & - & - & - & - & - & - & - \\
\hline AMD-2/9 & Jan-96 & -7.30 & - & - & - & - & - & - & - & - & - \\
\hline AMD-3/4 & 6/26/1997 & -9.40 & -78 & 41.0 & 7.2 & 61.9 & - & - & - & - & - \\
\hline AMD-3/6 & 6/26/1997 & -9.79 & -80 & 43.0 & 13.4 & 91.2 & - & - & - & - & - \\
\hline AMD-3/9 & $6 / 26 / 1997$ & -10.10 & -83 & 72.0 & 22.3 & 251.3 & - & - & - & - & - \\
\hline AMD-4/3 & 6/27/1997 & -9.49 & -73 & 55.0 & 17.5 & 146.6 & - & - & - & - & - \\
\hline AMD-4/4 & $6 / 27 / 1997$ & -9.60 & -79 & 53.0 & 17.8 & 145.0 & - & - & - & - & - \\
\hline AMD-4/9 & $6 / 27 / 1997$ & -9.94 & -77 & 106.0 & 26.5 & 453.8 & - & - & - & - & - \\
\hline AMD-4/10 & $6 / 26 / 1997$ & -8.20 & -58 & 4.8 & 24.1 & 18.3 & - & - & - & - & - \\
\hline
\end{tabular}


Table 1: Preliminary Isotope Data, Ages, and Recharge Temperatures calculated for Groundwater in the Forebay

\begin{tabular}{|c|c|c|c|c|c|c|c|c|c|c|c|}
\hline sample & collection date & $\delta^{18} \mathbf{O}$ & $\delta \mathbf{D}$ & ${ }^{3} \mathrm{H} \mathrm{pCi} / \mathrm{L}$ & ${ }^{3} \mathrm{H}^{3} \mathrm{He}$ age (yrs) & ${ }^{3} \mathrm{H}$ initial pCi/L & recharge $\mathrm{T}^{\circ} \mathrm{C}$ & excess air $\left(\mathrm{cc} / \mathrm{LH}_{2} \mathrm{O}\right)$ & ${ }^{14} \mathrm{C}$ pmc & $\delta^{13} \mathrm{C}$ per mil & DIC (mg/L l \\
\hline AMD-5/3 & 7/1/1997 & -7.94 & -59 & 27.0 & 22.2 & 93.5 & - & - & - & - & - \\
\hline AMD-5/4 & 7/1/1997 & -7.80 & -57 & 33.0 & 19.2 & 98.1 & - & - & - & - & - \\
\hline AMD-5/5 & $7 / 1 / 1997$ & -8.19 & -60 & 47.0 & 29.4 & 245.6 & - & - & - & - & - \\
\hline AMD-5/8 & $7 / 1 / 1997$ & -8.20 & -64 & 50.0 & 29.6 & 264.3 & - & - & - & - & - \\
\hline AMD-5/12 & $7 / 1 / 1997$ & -8.29 & -68 & 0.0 & $>40$ & - & - & - & - & - & - \\
\hline AMD-6/3 & $6 / 27 / 1997$ & -7.69 & -62 & 35.0 & 25.7 & 149.5 & - & - & - & - & - \\
\hline AMD-6/5 & 6/30/1997 & -8.21 & -67 & 34.0 & 27.3 & 155.6 & - & - & - & - & - \\
\hline AMD-6/8 & 6/30/1997 & -8.97 & -66 & 40.0 & 29.3 & 207.1 & - & - & - & - & - \\
\hline AMD-6/10 & $6 / 30 / 1997$ & -8.62 & -63 & 0.0 & $>40$ & - & & & & & \\
\hline AMD-6/13 & 6/30/1997 & -8.48 & -62 & 3.9 & $>40$ & - & - & - & - & - & - \\
\hline AMD-7/4 & $7 / 3 / 1997$ & -8.29 & -65 & 36.0 & 18.8 & 103.3 & - & - & - & - & - \\
\hline AMD-7/6 & 7/3/1997 & -9.25 & -69 & 44.0 & 19.9 & 135.2 & - & - & - & - & - \\
\hline AMD-7/8 & 7/3/1997 & -9.94 & -80 & 114.0 & 30.0 & 611.4 & - & - & - & - & - \\
\hline AMD-7/10 & 7/3/1997 & -10.34 & -78 & 144.0 & 27.6 & 677.8 & - & - & - & - & - \\
\hline AMD-9/1 & 9/12/1996 & - & - & - & 0.4 & - & 34 & 0.0006 & - & - & - \\
\hline AMD-9/1 & $10 / 1 / 1996$ & -7.33 & - & - & 0.4 & - & - & - & - & - & - \\
\hline AMD-9/1 & $10 / 2 / 1996$ & -7.27 & - & - & - & - & - & - & - & - & - \\
\hline AMD-9/1 & 10/3/1996 & -7.27 & - & - & - & - & - & - & - & - & - \\
\hline AMD-9/1 & 10/7/1996 & -7.37 & - & - & - & - & 32 & 0.0013 & - & - & - \\
\hline AMD-9/1 & $10 / 10 / 1996$ & - & - & - & - & - & 33 & 0.0006 & - & - & - \\
\hline AMD-9/1 & $10 / 11 / 1996$ & -7.73 & - & - & - & - & - & - & - & - & - \\
\hline AMD-9/1 & $10 / 16 / 1996$ & -10.79 & - & - & - & - & - & - & - & - & - \\
\hline AMD-9/1 & $10 / 31 / 1996$ & -11.28 & - & - & - & - & - & - & - & - & - \\
\hline AMD-9/1 & $12 / 4 / 1996$ & -11.03 & - & - & - & - & - & - & - & - & - \\
\hline AMD-9/1 & $12 / 31 / 1996$ & -7.73 & - & - & - & - & - & - & - & - & - \\
\hline AMD-9/1 & 10/8/1998 & -6.99 & - & - & - & - & - & - & - & - & - \\
\hline AMD-9/1 & $10 / 15 / 1998$ & -10.40 & - & - & - & - & - & - & - & - & - \\
\hline AMD-9/1 & $10 / 21 / 1998$ & -11.48 & - & - & - & - & - & - & - & - & - \\
\hline AMD-9/1 & $10 / 28 / 1998$ & -11.57 & - & - & - & - & - & - & - & - & - \\
\hline AMD-9/1 & $11 / 4 / 1998$ & -11.62 & - & - & - & - & - & - & - & - & - \\
\hline AMD-9/1 & $11 / 7 / 1998$ & -11.66 & - & - & - & - & - & - & - & - & - \\
\hline AMD-9/1 & $11 / 9 / 1998$ & -11.64 & - & - & - & - & - & - & - & - & - \\
\hline AMD-9/1 & $11 / 13 / 1998$ & -11.70 & - & - & - & - & - & - & - & - & - \\
\hline AMD-9/1 & $11 / 20 / 1998$ & -11.55 & - & - & - & - & - & - & - & - & - \\
\hline AMD-9/1 & $11 / 24 / 1998$ & -11.51 & - & - & - & - & - & - & - & - & - \\
\hline AMD-9/1 & $12 / 7 / 1998$ & -7.21 & - & - & - & - & - & - & - & - & - \\
\hline AMD-9/1 & $12 / 30 / 1998$ & -7.63 & - & - & - & - & - & - & - & - & - \\
\hline AMD-9/1 & $1 / 12 / 1999$ & -7.65 & - & - & - & - & - & - & - & - & - \\
\hline AMD-9/2 & 9/12/1996 & -7.58 & - & - & 2.7 & - & 20 & 0.0173 & - & - & - \\
\hline AMD-9/2 & $9 / 12 / 1996$ & -7.58 & - & - & - & - & - & - & - & - & - \\
\hline AMD-9/2 & $10 / 7 / 1996$ & - & - & - & - & - & 20 & 0.0195 & - & - & - \\
\hline AMD-9/2 & $10 / 31 / 1996$ & -7.65 & - & - & - & - & - & - & - & - & - \\
\hline AMD-9/2 & $12 / 4 / 1996$ & -7.68 & - & - & - & - & - & - & - & - & - \\
\hline
\end{tabular}


Table 1: Preliminary Isotope Data, Ages, and Recharge Temperatures calculated for Groundwater in the Forebay

\begin{tabular}{|c|c|c|c|c|c|c|c|c|c|c|c|}
\hline sample & collection date & $\delta^{18} \mathrm{O}$ & $\delta \mathbf{D}$ & ${ }^{3} \mathrm{H}$ pCi/L & ${ }^{3} \mathrm{H}-{ }^{3} \mathrm{He}$ age (yrs) & ${ }^{3} \mathrm{H}$ initial pCi/L & recharge $\mathrm{T}^{\circ} \mathrm{C}$ & excess air $\left(\mathrm{cc} / \mathrm{LH}_{2} \mathrm{O}\right)$ & ${ }^{14} \mathrm{C} \mathrm{pmc}$ & $\delta^{13} \mathrm{C}$ per mil & DIC (mg/L $\left.\mathrm{HCO}_{3}\right)$ \\
\hline AMD-9/2 & 12/31/1996 & -7.68 & - & - & - & - & - & - & - & - & - \\
\hline AMD-9/2 & 1/30/1997 & -7.94 & - & - & - & - & - & - & - & - & - \\
\hline AMD-9/2 & 2/18/1997 & -8.06 & - & - & - & - & - & - & - & - & - \\
\hline AMD-9/2 & $3 / 31 / 1997$ & -9.75 & - & - & - & - & - & - & - & - & - \\
\hline AMD-9/2 & 4/22/1997 & -9.68 & - & - & - & - & - & - & - & - & - \\
\hline AMD-9/2 & $5 / 20 / 1997$ & -8.62 & - & - & - & - & - & - & - & - & - \\
\hline AMD-9/2 & 6/18/1997 & -7.85 & - & - & - & - & - & - & - & - & - \\
\hline AMD-9/2 & $7 / 15 / 1997$ & -7.65 & - & - & - & - & - & - & - & - & - \\
\hline AMD-9/2 & 10/21/1997 & -10.08 & - & - & - & - & - & - & - & - & - \\
\hline AMD-9/2 & $11 / 12 / 1997$ & -10.08 & - & - & - & - & - & - & - & - & - \\
\hline AMD-9/2 & $12 / 16 / 1997$ & -9.44 & - & - & - & - & - & - & - & - & - \\
\hline AMD-9/2 & $12 / 30 / 1998$ & -7.48 & - & - & - & - & - & - & - & - & - \\
\hline AMD-9/2 & 1/5/1999 & -7.42 & - & - & - & - & - & - & - & - & - \\
\hline AMD-9/2 & 1/12/1999 & -7.45 & - & - & - & - & - & - & - & - & - \\
\hline AMD-9/2 & 1/25/1999 & -7.37 & - & - & - & - & - & - & - & - & - \\
\hline AMD-9/3 & $9 / 12 / 1996$ & -8.03 & - & - & - & - & - & - & - & - & - \\
\hline AMD-9/3 & $10 / 31 / 1996$ & -7.94 & - & - & - & - & - & - & - & - & - \\
\hline AMD-9/3 & $12 / 4 / 1996$ & -7.97 & - & - & - & - & - & - & - & - & - \\
\hline AMD-9/3 & $12 / 31 / 1996$ & -7.96 & - & - & - & - & - & - & - & - & - \\
\hline AMD-9/3 & 1/30/1997 & -7.90 & - & - & - & - & - & - & - & - & - \\
\hline AMD-9/3 & $3 / 31 / 1997$ & -7.93 & - & - & - & - & - & - & - & - & - \\
\hline AMD-9/3 & 4/22/1997 & -7.91 & - & - & - & - & - & - & - & - & - \\
\hline AMD-9/3 & $6 / 18 / 1997$ & -7.84 & - & - & - & - & - & - & - & - & - \\
\hline AMD-9/3 & 7/15/1997 & -7.83 & - & - & - & - & - & - & - & - & - \\
\hline AMD-9/3 & 9/24/1997 & -7.73 & - & - & - & - & - & - & - & - & - \\
\hline AMD-9/3 & $10 / 21 / 1997$ & -7.85 & - & - & - & - & - & - & - & - & - \\
\hline AMD-9/3 & $11 / 12 / 1997$ & -7.86 & - & - & - & - & - & - & - & - & - \\
\hline AMD-9/3 & $12 / 16 / 1997$ & -7.98 & - & - & - & - & - & - & - & - & - \\
\hline AMD-9/4 & 9/12/1996 & -7.73 & - & - & 25.3 & - & - & - & - & - & - \\
\hline AMD-9/4 & $1 / 30 / 1997$ & -8.07 & - & - & - & - & - & - & - & - & - \\
\hline AMD-9/4 & $3 / 31 / 1997$ & -7.79 & - & - & - & - & - & - & - & - & - \\
\hline AMD-9/4 & $6 / 18 / 1997$ & -7.74 & - & - & - & - & - & - & - & - & - \\
\hline AMD-9/4 & $7 / 15 / 1997$ & -7.72 & - & - & - & - & - & - & - & - & - \\
\hline AMD-9/4 & $8 / 26 / 1997$ & -7.76 & - & - & - & - & - & - & - & - & - \\
\hline AMD-9/4 & 9/24/1997 & -7.77 & - & - & - & - & - & - & - & - & - \\
\hline AMD-9/4 & $10 / 21 / 1997$ & -7.80 & - & - & - & - & - & - & - & - & - \\
\hline AMD-9/4 & $11 / 12 / 1997$ & -7.92 & - & - & - & - & - & - & - & - & - \\
\hline AMD-9/4 & $12 / 16 / 1997$ & -7.83 & - & - & - & - & - & - & - & - & - \\
\hline AMD-10/1 & $10 / 15 / 1998$ & -7.24 & - & - & - & - & - & - & - & - & - \\
\hline AMD-10/1 & $10 / 28 / 1998$ & -7.20 & - & - & - & - & - & - & - & - & - \\
\hline AMD-10/1 & 11/9/1998 & -7.23 & - & - & - & - & - & - & - & - & - \\
\hline AMD-10/1 & $11 / 25 / 1998$ & -7.31 & - & - & - & - & - & - & - & - & - \\
\hline AMD-10/1 & $12 / 8 / 1998$ & -7.54 & - & - & - & - & - & - & - & - & - \\
\hline AMD-10/1 & $12 / 21 / 1998$ & -7.97 & - & - & - & - & - & - & - & - & - \\
\hline AMD-10/1 & 1/5/1999 & -8.42 & - & - & - & - & - & - & - & - & - \\
\hline
\end{tabular}


Table 1: Preliminary Isotope Data, Ages, and Recharge Temperatures calculated for Groundwater in the Forebay

\begin{tabular}{|c|c|c|c|c|c|c|c|c|c|c|c|}
\hline sample & collection date & $\delta^{18} \mathrm{O}$ & $\delta \mathbf{D}$ & ${ }^{3} \mathrm{H}$ pCi/L & ${ }^{3} \mathrm{H}-{ }^{3} \mathrm{He}$ age (yrs) & ${ }^{3} \mathrm{H}$ initial pCi/L & recharge $\mathrm{T}^{\circ} \mathrm{C}$ & excess air $\left(\mathrm{cc} / \mathrm{LH}_{2} \mathrm{O}\right)$ & ${ }^{14} \mathrm{C} \mathrm{pmc}$ & $\delta^{13} \mathrm{C}$ per mil & $\mathrm{DIC}\left(\mathrm{mg} / \mathrm{L} \mathrm{HCO}_{3}\right)$ \\
\hline AMD-10/1 & 1/18/1999 & -8.67 & - & - & - & - & - & - & - & - & - \\
\hline AMD-10/1 & 2/1/1999 & -8.83 & - & - & - & - & - & - & - & - & - \\
\hline AMD-10/2 & $10 / 15 / 1998$ & -7.49 & - & - & - & - & - & - & - & - & - \\
\hline AMD-10/2 & $10 / 28 / 1998$ & -7.44 & - & - & - & - & - & - & - & - & - \\
\hline AMD-10/2 & 11/9/1998 & -7.45 & - & - & - & - & - & - & - & - & - \\
\hline AMD-10/2 & $11 / 25 / 1998$ & -7.42 & - & - & - & - & - & - & - & - & - \\
\hline AMD-10/2 & 12/8/1998 & -7.40 & - & - & - & - & - & - & - & - & - \\
\hline AMD-10/2 & $12 / 21 / 1998$ & -7.37 & - & - & - & - & - & - & - & - & - \\
\hline AMD-10/2 & 1/5/1999 & -7.39 & - & - & - & - & - & - & - & - & - \\
\hline AMD-10/2 & $1 / 18 / 1999$ & -7.38 & - & - & - & - & - & - & - & - & - \\
\hline AMD-10/2 & 2/1/1999 & -7.43 & - & - & - & - & - & - & - & - & - \\
\hline AMD-10/3 & $10 / 28 / 1998$ & -8.07 & - & - & - & - & - & - & - & - & - \\
\hline AMD-10/3 & $11 / 25 / 1998$ & -8.04 & - & - & - & - & - & - & - & - & - \\
\hline AMD-10/3 & $12 / 21 / 1998$ & -7.82 & - & - & - & - & - & - & - & - & - \\
\hline AMD-10/3 & $1 / 18 / 1999$ & -7.80 & - & - & - & - & - & - & - & - & - \\
\hline AMD-10/4 & $10 / 28 / 1998$ & -8.46 & - & - & - & - & - & - & - & - & - \\
\hline AMD-10/4 & $11 / 25 / 1998$ & -8.50 & - & - & - & - & - & - & - & - & - \\
\hline AMD-10/4 & $12 / 21 / 1998$ & -8.44 & - & - & - & - & - & - & - & - & - \\
\hline AMD-10/4 & $1 / 18 / 1999$ & -8.48 & - & - & - & - & - & - & - & - & - \\
\hline AMD-11/1 & $10 / 29 / 1998$ & -7.93 & - & - & - & - & - & - & - & - & - \\
\hline AMD-11/1 & $11 / 9 / 1998$ & -7.87 & - & - & - & - & - & - & - & - & - \\
\hline AMD-11/1 & 11/25/1998 & -7.83 & - & - & - & - & - & - & - & - & - \\
\hline AMD-11/1 & $12 / 8 / 1998$ & -7.76 & - & - & - & - & - & - & - & - & - \\
\hline AMD-11/1 & $12 / 21 / 1998$ & -7.68 & - & - & - & - & - & - & - & - & - \\
\hline AMD-11/1 & 1/5/1999 & -7.62 & - & - & - & - & - & - & - & - & - \\
\hline AMD-11/1 & 2/1/1999 & -7.60 & - & - & - & - & - & - & - & - & - \\
\hline AMD-11/2 & $10 / 29 / 1998$ & -7.88 & - & - & - & - & - & - & - & - & - \\
\hline AMD-11/2 & $11 / 9 / 1998$ & -7.88 & - & - & - & - & - & - & - & - & - \\
\hline AMD-11/2 & $11 / 25 / 1998$ & -7.85 & - & - & - & - & - & - & - & - & - \\
\hline AMD-11/2 & $12 / 8 / 1998$ & -7.89 & - & - & - & - & - & - & - & - & - \\
\hline AMD-11/2 & $12 / 21 / 1998$ & -7.84 & - & - & - & - & - & - & - & - & - \\
\hline AMD-11/2 & 1/5/1999 & -7.87 & - & - & - & - & - & - & - & - & - \\
\hline AMD-11/2 & 2/1/1999 & -7.89 & - & - & - & - & - & - & - & - & - \\
\hline AMD-11/3 & $10 / 29 / 1998$ & -7.75 & - & - & - & - & - & - & - & - & - \\
\hline AMD-11/3 & $11 / 25 / 1998$ & -7.72 & - & - & - & - & - & - & - & - & - \\
\hline AMD-11/3 & $12 / 21 / 1998$ & -7.73 & - & - & - & - & - & - & - & - & - \\
\hline AMD-11/3 & 1/18/1999 & -7.72 & - & - & - & - & - & - & - & - & - \\
\hline AMD-11/4 & $10 / 29 / 1998$ & -7.94 & - & - & - & - & - & - & - & - & - \\
\hline AMD-11/4 & $11 / 25 / 1998$ & -7.94 & - & - & - & - & - & - & - & - & - \\
\hline AMD-11/4 & $12 / 21 / 1998$ & -7.92 & - & - & - & - & - & - & - & - & - \\
\hline AMD-11/4 & 1/18/1999 & -7.95 & - & - & - & - & - & - & - & - & - \\
\hline
\end{tabular}


Table 1: Preliminary Isotope Data, Ages, and Recharge Temperatures calculated for Groundwater in the Forebay

\begin{tabular}{|c|c|c|c|c|c|c|c|c|c|c|c|}
\hline sample & collection date & $\delta^{18} \mathrm{O}$ & $\delta \mathbf{D}$ & ${ }^{3} \mathrm{H} \mathrm{pCi} / \mathrm{L}$ & ${ }^{3} \mathrm{H}-{ }^{3} \mathrm{He}$ age (yrs) & ${ }^{3} \mathrm{H}$ initial pCi/L & recharge $\mathbf{T}^{\circ} \mathrm{C}$ & excess air $\left(\mathrm{cc} / \mathrm{LH}_{2} \mathrm{O}\right)$ & ${ }^{14} \mathrm{C}$ pmc & $\delta^{13} \mathrm{C}$ per mil & DIC (mg/L HC \\
\hline ABS-2 & 3/8/1995 & -9.40 & -71 & 25.00 & 4.7 & 32.0 & 19 & 0.0113 & 102 & -11.7 & 130 \\
\hline ABS-2 & 9/26/1996 & - & - & - & - & - & 21 & 0.0241 & - & - & - \\
\hline ABS-2 & $10 / 11 / 1996$ & -7.90 & - & - & - & - & - & - & - & - & - \\
\hline ABS-2 & $11 / 20 / 1996$ & -8.84 & - & - & - & - & - & - & - & - & - \\
\hline ABS-2 & $12 / 31 / 1996$ & -9.52 & - & - & - & - & - & - & - & - & - \\
\hline ABS-2 & 2/13/1997 & -8.88 & - & - & - & - & - & - & - & - & - \\
\hline ABS-2 & 4/2/1997 & -8.47 & - & - & - & - & - & - & - & - & - \\
\hline ABS-2 & $4 / 25 / 1997$ & -8.15 & - & - & - & - & - & - & - & - & - \\
\hline Anaheim Lake & $6 / 1 / 1995$ & -7.30 & - & - & - & - & - & - & - & - & - \\
\hline Anaheim Lake & 8/30/1995 & -6.90 & - & - & - & - & - & - & - & - & - \\
\hline Anaheim Lake & 9/20/1995 & -6.60 & - & - & - & - & - & - & - & - & - \\
\hline Anaheim Lake & $2 / 21 / 1996$ & -7.33 & - & - & - & - & - & - & - & - & - \\
\hline Anaheim Lake & 4/17/1996 & -7.71 & - & - & - & - & - & - & - & - & - \\
\hline Anaheim Lake & 6/19/1996 & -7.21 & - & - & - & - & - & - & - & - & - \\
\hline Anaheim Lake & 7/17/1996 & -7.77 & - & - & - & - & - & - & - & - & - \\
\hline Anaheim Lake & $8 / 21 / 1996$ & -7.43 & - & - & - & - & - & - & - & - & - \\
\hline Anaheim Lake & $10 / 2 / 1996$ & -11.30 & - & - & - & - & - & - & - & - & - \\
\hline Anaheim Lake Bottom & $10 / 11 / 1996$ & -11.20 & - & - & - & - & - & - & - & - & - \\
\hline Anaheim Lake Mid Depth & $10 / 11 / 1996$ & -11.11 & - & - & - & - & - & - & - & - & - \\
\hline Anaheim Lake Top & $10 / 11 / 1996$ & -11.18 & - & - & - & - & - & - & - & - & - \\
\hline Anaheim Lake Bottom & $10 / 11 / 1996$ & -11.30 & - & - & - & - & - & - & - & - & - \\
\hline Anaheim Lake Top & $10 / 11 / 1996$ & -11.24 & - & - & - & - & - & - & - & - & - \\
\hline Anaheim Lake Bottom & 10/11/1996 & -11.21 & - & - & - & - & - & - & - & - & - \\
\hline Anaheim Lake Mid Depth & $10 / 11 / 1996$ & -11.23 & - & - & - & - & - & - & - & - & - \\
\hline Anaheim Lake Mid Depth & $10 / 11 / 1996$ & -11.30 & - & - & - & - & - & - & - & - & - \\
\hline Anaheim Lake Mid Depth & $10 / 16 / 1996$ & -11.25 & - & - & - & - & - & - & - & - & - \\
\hline Anaheim Lake Top & $10 / 16 / 1996$ & -11.22 & - & - & - & - & - & - & - & - & - \\
\hline Anaheim Lake Mid Depth & $10 / 16 / 1996$ & -11.29 & - & - & - & - & - & - & - & - & - \\
\hline Anaheim Lake Mid Depth & $10 / 16 / 1996$ & -11.26 & - & - & - & - & - & - & - & - & - \\
\hline $\mathrm{CB}-1 / 2$ & 7/1/1997 & -6.43 & -45 & 26.0 & 10.9 & 47.6 & - & - & - & - & - \\
\hline CB- $1 / 3$ & $7 / 1 / 1997$ & -8.65 & -65 & 65.0 & 24.8 & 259.3 & - & - & - & - & - \\
\hline CB- $1 / 5$ & $7 / 1 / 1997$ & -10.27 & -82 & 102.0 & 24.4 & 400.3 & - & - & - & - & - \\
\hline CB- $1 / 6$ & $7 / 1 / 1997$ & -8.47 & -59 & 5.1 & 19.1 & 14.9 & - & - & - & - & - \\
\hline EOCW-E & $4 / 25 / 1995$ & -7.10 & -55 & 27.00 & 23.9 & 102.0 & 12 & 0.0308 & 78 & -11.5 & 196 \\
\hline F-AIRP & 3/8/1995 & -7.90 & -52 & 15.00 & - & - & 16 & 0.0033 & 62 & -11.8 & 206 \\
\hline F-AIRP & $8 / 28 / 1995$ & -7.99 & - & - & - & - & - & - & - & - & - \\
\hline FKIM & 6/30/1997 & -9.30 & -73 & 32.0 & 14.2 & 71.0 & - & - & - & - & - \\
\hline F4 & $10 / 16 / 1997$ & -9.26 & - & 17.2 & - & - & - & - & - & - & - \\
\hline F8 & $10 / 6 / 1997$ & -9.37 & - & 23.5 & - & - & - & - & - & - & - \\
\hline HG-1 & 3/9/1995 & -8.00 & -61 & 18.00 & 1.3 & 19.0 & 24 & 0.0014 & 105 & -10.9 & 185 \\
\hline
\end{tabular}


Table 1: Preliminary Isotope Data, Ages, and Recharge Temperatures calculated for Groundwater in the Forebay

\begin{tabular}{|c|c|c|c|c|c|c|c|c|c|c|c|}
\hline sample & collection date & $\delta^{18} \mathrm{O}$ & $\delta \mathbf{D}$ & ${ }^{3} \mathrm{H} \mathrm{pCi} / \mathrm{L}$ & ${ }^{3} \mathrm{H}-{ }^{3} \mathrm{He}$ age (vrs) & ${ }^{3} \mathrm{H}$ initial pCi/L & recharge $\mathrm{T}^{\circ} \mathrm{C}$ & excess air $\left(\mathrm{cc} / \mathrm{LH}_{2} \mathrm{O}\right)$ & ${ }^{14} \mathrm{C} \mathrm{pmc}$ & $\delta^{13} \mathrm{C}$ per mil & DIC (mg/L $\left.\mathrm{HCO}_{3}\right)$ \\
\hline HG-1 & $6 / 26 / 1995$ & -7.50 & -51 & - & - & - & 19 & 0 & 104 & -12.6 & 236 \\
\hline HG-1 & 9/19/1995 & -7.46 & - & - & - & - & - & - & - & - & - \\
\hline HG-1 & $10 / 17 / 1995$ & -7.45 & - & - & - & - & - & - & - & - & - \\
\hline HG-1 & $11 / 14 / 1995$ & -7.49 & - & - & - & - & - & - & - & - & - \\
\hline HG-1 & $12 / 12 / 1995$ & -7.73 & - & - & - & - & - & - & - & - & - \\
\hline HG-1 & 1/16/1996 & -7.86 & - & - & - & - & - & - & - & - & - \\
\hline HG-1 & 2/22/1996 & -7.27 & - & - & - & - & - & - & - & - & - \\
\hline HG-1 & 3/19/1996 & -7.53 & - & - & - & - & - & - & - & - & - \\
\hline HG-1 & $4 / 16 / 1996$ & -7.51 & - & - & - & - & - & - & - & - & - \\
\hline HG-1 & $5 / 14 / 1996$ & -8.00 & - & - & - & - & - & - & - & - & - \\
\hline HG-1 & $7 / 16 / 1996$ & -8.12 & - & - & - & - & 27 & 0 & - & - & - \\
\hline HG-1 & $5 / 23 / 1996$ & -8.37 & - & - & - & - & 24 & 0.0006 & - & - & - \\
\hline HG-1 & $6 / 18 / 1996$ & -7.97 & - & - & - & - & - & - & - & - & - \\
\hline HG-1 & $8 / 28 / 1996$ & -8.05 & - & - & - & - & - & - & - & - & - \\
\hline KBS-1 & 9/29/1996 & -7.70 & - & - & - & - & - & - & - & - & - \\
\hline KBS-1 & $10 / 16 / 1996$ & -7.51 & - & - & - & - & - & - & - & - & - \\
\hline KBS-1 & $10 / 31 / 1996$ & -7.65 & - & - & - & - & - & - & - & - & - \\
\hline KBS-1 & $11 / 20 / 1996$ & -7.70 & - & - & - & - & - & - & - & - & - \\
\hline KBS-1 & $12 / 30 / 1996$ & -8.24 & - & - & - & - & - & - & - & - & - \\
\hline KBS-1 & $1 / 27 / 1997$ & -6.78 & - & - & - & - & - & - & - & - & - \\
\hline KBS-1 & 2/21/1997 & -7.12 & - & - & - & - & - & - & - & - & - \\
\hline KBS-1 & 4/1/1997 & -7.42 & - & - & - & - & - & - & - & - & - \\
\hline KBS-1 & 4/24/1997 & -8.71 & - & - & - & - & - & - & - & - & - \\
\hline KBS-1 & $5 / 22 / 1997$ & -7.61 & - & - & - & - & - & - & - & - & - \\
\hline KBS-1 & 6/19/1997 & -7.68 & - & - & - & - & - & - & - & - & - \\
\hline KBS-1 & $7 / 16 / 1997$ & -7.74 & - & - & - & - & - & - & - & - & - \\
\hline KBS-1 & 10/4/1997 & -8.03 & - & - & - & - & - & - & - & - & - \\
\hline KBS-1 & 10/22/1997 & -8.13 & - & - & - & - & - & - & - & - & - \\
\hline KBS-1 & $11 / 14 / 1997$ & -8.53 & - & - & - & - & - & - & - & - & - \\
\hline KBS-1 & $12 / 17 / 1997$ & -8.10 & - & - & - & - & - & - & - & - & - \\
\hline KBS-1 & $10 / 8 / 1998$ & -7.23 & - & - & - & - & - & - & - & - & - \\
\hline KBS-1 & $10 / 16 / 1998$ & -11.35 & - & - & - & - & - & - & - & - & - \\
\hline KBS-1 & $10 / 21 / 1998$ & -11.60 & - & - & - & - & - & - & - & - & - \\
\hline KBS-1 & $10 / 28 / 1998$ & -10.95 & - & - & - & - & - & - & - & - & - \\
\hline KBS-1 & 11/4/1998 & -10.84 & - & - & - & - & - & - & - & - & - \\
\hline KBS-1 & $11 / 9 / 1998$ & -9.17 & - & - & - & - & - & - & - & - & - \\
\hline KBS-1 & $11 / 16 / 1998$ & -7.89 & - & - & - & - & - & - & - & - & - \\
\hline KBS-1 & $11 / 25 / 1998$ & -7.67 & - & - & - & - & - & - & - & - & - \\
\hline KBS-1 & 12/4/1998 & -7.98 & - & - & - & - & - & - & - & - & - \\
\hline KBS-1 & $12 / 10 / 1998$ & -10.86 & - & - & - & - & - & - & - & - & - \\
\hline KBS-1 & $12 / 15 / 1998$ & -7.83 & - & - & - & - & - & - & - & - & - \\
\hline KBS-1 & $12 / 22 / 1998$ & -7.50 & - & - & - & - & - & - & - & - & - \\
\hline KBS-1 & $12 / 29 / 1998$ & -7.65 & - & - & - & - & - & - & - & - & - \\
\hline KBS-1 & 1/11/1999 & -8.40 & - & - & - & - & - & - & - & - & - \\
\hline KBS-1 & 1/19/1999 & -7.94 & - & - & - & - & - & - & - & - & - \\
\hline KBS-1 & 1/25/1999 & -7.57 & - & - & - & - & - & - & - & - & - \\
\hline
\end{tabular}


Table 1: Preliminary Isotope Data, Ages, and Recharge Temperatures calculated for Groundwater in the Forebay

\begin{tabular}{|c|c|c|c|c|c|c|c|c|c|c|c|}
\hline $\begin{array}{l}\text { sample } \\
\text { KBS-1 }\end{array}$ & $\begin{array}{c}\text { collection date } \\
1 / 28 / 1999\end{array}$ & $\begin{array}{l}\delta^{18} \mathbf{O} \\
-7.81\end{array}$ & $\begin{array}{c}\delta \mathbf{D} \\
-\end{array}$ & $\begin{array}{c}{ }^{3} \mathrm{H} \text { pCi/L } \\
-\end{array}$ & $\begin{array}{c}{ }^{3} \mathrm{H}-{ }^{3} \mathrm{He} \text { age (vrs) } \\
-\end{array}$ & $\begin{array}{c}{ }^{3} \mathrm{H} \text { initial pCi/L } \\
-\end{array}$ & $\begin{array}{c}\text { recharge } \mathbf{T}^{\circ} \mathbf{C} \\
-\end{array}$ & $\begin{array}{c}\text { excess air }\left(\mathrm{cc} / \mathrm{LH}_{2} \mathrm{O}\right) \\
-\end{array}$ & $\begin{array}{c}{ }^{14} \mathrm{C} \text { pmc } \\
-\end{array}$ & $\begin{array}{c}\delta^{13} \mathrm{C} \text { per mil } \\
-\end{array}$ & $\begin{array}{c}\text { DIC (mg/L HC } \\
-\end{array}$ \\
\hline KBS-2/1 & $10 / 7 / 1998$ & -11.38 & - & - & - & - & - & - & - & - & - \\
\hline KBS-2/1 & $10 / 16 / 1998$ & -11.74 & - & - & - & - & - & - & - & - & - \\
\hline KBS-2/1 & $10 / 21 / 1998$ & -11.64 & - & - & - & - & - & - & - & - & - \\
\hline KBS-2/1 & 12/9/1998 & -7.26 & - & - & - & - & - & - & - & - & - \\
\hline KBS-2/1 & $12 / 22 / 1998$ & -7.56 & - & - & - & - & - & - & - & - & - \\
\hline KBS-2/1 & $12 / 29 / 1998$ & -7.57 & - & - & - & - & - & - & - & - & - \\
\hline KBS-2/1 & 1/13/1999 & -7.77 & - & - & - & - & - & - & - & - & - \\
\hline KBS-2/1 & 1/20/1999 & -7.93 & - & - & - & - & - & - & - & - & - \\
\hline KBS-2/1 & 2/1/1999 & -7.89 & - & - & - & - & - & - & - & - & - \\
\hline KBS- $2 / 2$ & $7 / 30 / 1996$ & - & - & - & - & - & 23 & 0.0104 & - & - & - \\
\hline $\mathrm{KBS}-2 / 2$ & 9/30/1996 & -7.63 & - & - & - & - & - & - & - & - & - \\
\hline KBS- $2 / 2$ & $11 / 20 / 1996$ & -7.55 & - & - & - & - & - & - & - & - & - \\
\hline KBS- $2 / 2$ & $12 / 16 / 1996$ & -7.68 & - & - & - & - & - & - & - & - & - \\
\hline KBS- $2 / 2$ & 2/20/1997 & -7.06 & - & - & - & - & - & - & - & - & - \\
\hline $\mathrm{KBS}-2 / 2$ & $4 / 25 / 1997$ & -7.24 & - & - & - & - & - & - & - & - & - \\
\hline KBS-2/2 & $5 / 22 / 1997$ & -7.39 & - & - & - & - & - & - & - & - & - \\
\hline KBS- $2 / 2$ & 6/20/1997 & -7.77 & - & - & - & - & - & - & - & - & - \\
\hline KBS-2/2 & $8 / 22 / 1997$ & -7.78 & - & - & - & - & - & - & - & - & - \\
\hline KBS- $2 / 2$ & 9/26/1997 & -7.82 & - & - & - & - & - & - & - & - & - \\
\hline KBS- $2 / 2$ & $10 / 24 / 1997$ & -8.01 & - & - & - & - & - & - & - & - & - \\
\hline KBS- $2 / 2$ & $11 / 25 / 1997$ & -8.10 & - & - & - & - & - & - & - & - & - \\
\hline KBS- $2 / 2$ & 10/7/1998 & -7.15 & - & - & - & - & - & - & - & - & - \\
\hline KBS- $2 / 2$ & $10 / 16 / 1998$ & -7.10 & - & - & - & - & - & - & - & - & - \\
\hline KBS- $2 / 2$ & $10 / 21 / 1998$ & -7.18 & - & - & - & - & - & - & - & - & - \\
\hline KBS- $2 / 2$ & 12/9/1998 & -8.84 & - & - & - & - & - & - & - & - & - \\
\hline KBS- $2 / 2$ & $12 / 14 / 1998$ & -10.47 & - & - & - & - & - & - & - & - & - \\
\hline KBS-2/2 & $12 / 22 / 1998$ & -11.59 & - & - & - & - & - & - & - & - & - \\
\hline KBS-2/2 & $12 / 29 / 1998$ & -11.37 & - & - & - & - & - & - & - & - & - \\
\hline KBS- $2 / 2$ & 1/13/1999 & -9.52 & - & - & - & - & - & - & - & - & - \\
\hline KBS- $2 / 2$ & 1/20/1999 & -9.30 & - & - & - & - & - & - & - & - & - \\
\hline KBS- $2 / 2$ & $1 / 28 / 1999$ & -8.79 & - & - & - & - & - & - & - & - & - \\
\hline KBS-3/1 & $10 / 8 / 1998$ & -11.08 & - & - & - & - & - & - & - & - & - \\
\hline KBS-3/1 & $10 / 16 / 1998$ & -11.75 & - & - & - & - & - & - & - & - & - \\
\hline KBS-3/1 & 10/21/1998 & -11.70 & - & - & - & - & - & - & - & - & - \\
\hline KBS-3/1 & $10 / 28 / 1998$ & -11.74 & - & - & - & - & - & - & - & - & - \\
\hline KBS-3/1 & 12/4/1998 & -9.83 & - & - & - & - & - & - & - & - & - \\
\hline KBS-3/1 & $12 / 10 / 1998$ & -7.33 & - & - & - & - & - & - & - & - & - \\
\hline KBS-3/1 & $12 / 16 / 1998$ & -7.45 & - & - & - & - & - & - & - & - & - \\
\hline KBS-3/1 & $12 / 22 / 1998$ & -7.55 & - & - & - & - & - & - & - & - & - \\
\hline KBS-3/1 & $12 / 29 / 1998$ & -7.69 & - & - & - & - & - & - & - & - & - \\
\hline KBS-3/1 & 1/11/1999 & -7.76 & - & - & - & - & - & - & - & - & - \\
\hline KBS-3/1 & 1/18/1999 & -7.87 & - & - & - & - & - & - & - & - & - \\
\hline
\end{tabular}


Table 1: Preliminary Isotope Data, Ages, and Recharge Temperatures calculated for Groundwater in the Forebay

\begin{tabular}{|c|c|c|c|c|c|c|c|c|c|c|c|}
\hline sample & collection date & $\delta^{18} \mathrm{O}$ & $\delta \mathbf{D}$ & ${ }^{3} \mathrm{H} \mathrm{pCi} / \mathrm{L}$ & ${ }^{3} \mathrm{H}-{ }^{3} \mathrm{He}$ age (vrs) & ${ }^{3} \mathrm{H}$ initial pCi/L & recharge $\mathrm{T}^{\circ} \mathrm{C}$ & excess air $\left(\mathrm{cc} / \mathrm{LH}_{2} \mathrm{O}\right)$ & ${ }^{14} \mathrm{C} \mathrm{pmc}$ & $\delta^{13} \mathrm{C}$ per mil & DIC (mg/L $\left.\mathrm{HCO}_{3}\right)$ \\
\hline KBS-4 & $9 / 25 / 1996$ & - & - & - & - & - & 27 & 0.0037 & - & - & - \\
\hline KBS-4 & $12 / 4 / 1996$ & -7.36 & - & - & - & - & - & - & - & - & - \\
\hline KBS-4 & $12 / 30 / 1996$ & -8.26 & - & - & - & - & - & - & - & - & - \\
\hline KBS-4 & $10 / 31 / 1996$ & -7.60 & - & - & - & - & - & - & - & - & - \\
\hline KBS-4 & 1/27/1997 & -7.56 & - & - & - & - & - & - & - & - & - \\
\hline KBS-4 & 2/21/1997 & -6.86 & - & - & - & - & - & - & - & - & - \\
\hline KBS-4 & 4/1/1997 & -7.19 & - & - & - & - & - & - & - & - & - \\
\hline KBS-4 & 4/24/1997 & -7.21 & - & - & - & - & - & - & - & - & - \\
\hline KBS-4 & $5 / 22 / 1997$ & -7.76 & - & - & - & - & - & - & - & - & - \\
\hline KBS-4 & 6/19/1997 & -7.90 & - & - & - & - & - & - & - & - & - \\
\hline KBS-4 & $7 / 16 / 1997$ & -7.63 & - & - & - & - & - & - & - & - & - \\
\hline KBS-4 & 8/23/1997 & -7.57 & - & - & - & - & - & - & - & - & - \\
\hline KBS-4 & 9/26/1997 & -7.62 & - & - & - & - & - & - & - & - & - \\
\hline KBS-4 & $10 / 22 / 1997$ & -8.09 & - & - & - & - & - & - & - & - & - \\
\hline KBS-4 & 11/13/1997 & -7.76 & - & - & - & - & - & - & - & - & - \\
\hline KBS-4 & $12 / 17 / 1997$ & -7.70 & - & - & - & - & - & - & - & - & - \\
\hline KBS-4 & 10/8/1998 & -6.67 & - & - & - & - & - & - & - & - & - \\
\hline KBS-4 & $10 / 16 / 1998$ & -6.77 & - & - & - & - & - & - & - & - & - \\
\hline KBS-4 & $10 / 21 / 1998$ & -6.64 & - & - & - & - & - & - & - & - & - \\
\hline KBS-4 & $10 / 28 / 1998$ & -6.53 & - & - & - & - & - & - & - & - & - \\
\hline KBS-4 & 11/4/1998 & -6.45 & - & - & - & - & - & - & - & - & - \\
\hline KBS-4 & 11/9/1998 & -6.43 & - & - & - & - & - & - & - & - & - \\
\hline KBS-4 & $11 / 16 / 1998$ & -6.38 & - & - & - & - & - & - & - & - & - \\
\hline KBS-4 & $11 / 25 / 1998$ & -6.37 & - & - & - & - & - & - & - & - & - \\
\hline KBS-4 & $12 / 4 / 1998$ & -7.14 & - & - & - & - & - & - & - & - & - \\
\hline KBS-4 & $12 / 10 / 1998$ & -7.34 & - & - & - & - & - & - & - & - & - \\
\hline KBS-4 & $12 / 16 / 1998$ & -6.98 & - & - & - & - & - & - & - & - & - \\
\hline KBS-4 & $12 / 29 / 1998$ & -6.99 & - & - & - & - & - & - & - & - & - \\
\hline KBS-4 & 1/11/1999 & -7.15 & - & - & - & - & - & - & - & - & - \\
\hline KBS-4 & $1 / 19 / 1999$ & -7.28 & - & - & - & - & - & - & - & - & - \\
\hline OCWD-KB1 & $6 / 22 / 1995$ & -6.90 & -54 & 20.00 & -0.3 & - & 19 & 0.0132 & 103 & -9.3 & 212 \\
\hline OCWD-KB1 & 7/16/1996 & -7.87 & - & - & 0.1 & - & 27 & 0.0014 & - & - & - \\
\hline OCWD-KB1 & $5 / 29 / 1996$ & - & - & - & - & - & 23 & 0.0034 & - & - & - \\
\hline OCWD-KB1 & 9/23/1996 & - & - & - & - & - & 26 & 0.0042 & - & - & - \\
\hline OCWD-KB1 & $10 / 31 / 1996$ & -7.62 & - & - & - & - & - & - & - & - & - \\
\hline OCWD-KB1 & $11 / 21 / 1996$ & -7.63 & - & - & - & - & - & - & - & - & - \\
\hline OCWD-KB1 & $12 / 3 / 1996$ & -7.71 & - & - & - & - & - & - & - & - & - \\
\hline OCWD-KB1 & $12 / 30 / 1996$ & -8.39 & - & - & - & - & - & - & - & - & - \\
\hline OCWD-KB1 & 1/27/1997 & -6.89 & - & - & - & - & - & - & - & - & - \\
\hline OCWD-KB1 & 2/19/1997 & -7.87 & - & - & - & - & - & - & - & - & - \\
\hline OCWD-KB1 & 4/1/1997 & -7.31 & - & - & - & - & - & - & - & - & - \\
\hline OCWD-KB1 & $4 / 21 / 1997$ & -7.27 & - & - & - & - & - & - & - & - & - \\
\hline OCWD-KB1 & $5 / 19 / 1997$ & -7.55 & - & - & - & - & - & - & - & - & - \\
\hline OCWD-KB1 & 6/19/1997 & -7.93 & - & - & - & - & - & - & - & - & - \\
\hline OCWD-KB1 & $7 / 14 / 1997$ & -7.83 & - & - & - & - & - & - & - & - & - \\
\hline OCWD-KB1 & $8 / 26 / 1997$ & -7.64 & - & - & - & - & - & - & - & - & - \\
\hline
\end{tabular}


Table 1: Preliminary Isotope Data, Ages, and Recharge Temperatures calculated for Groundwater in the Forebay

\begin{tabular}{|c|c|c|c|c|c|c|c|c|c|c|c|}
\hline sample & collection date & $\delta^{18} \mathrm{O}$ & $\delta \mathbf{D}$ & ${ }^{3} \mathrm{H}$ pCi/L & ${ }^{3} \mathrm{H}-{ }^{3} \mathrm{He}$ age (vrs) & ${ }^{3} \mathrm{H}$ initial pCi/L & recharge $\mathrm{T}^{\circ} \mathrm{C}$ & excess air $\left(\mathrm{cc} / \mathrm{LH}_{2} \mathrm{O}\right)$ & ${ }^{14} \mathrm{C} \mathrm{pmc}$ & $\delta^{13} \mathrm{C}$ per mil & DIC (mg/L $\left.\mathrm{HCO}_{3}\right)$ \\
\hline OCWD-KB1 & 9/22/1997 & -8.14 & - & - & - & - & - & - & - & - & - \\
\hline OCWD-KB1 & 11/13/1997 & -8.14 & - & - & - & - & - & - & - & - & - \\
\hline OCWD-KB1 & $12 / 15 / 1997$ & -7.93 & - & - & - & - & - & - & - & - & - \\
\hline OCWD-KB1 & 10/8/1998 & -7.04 & - & - & - & - & - & - & - & - & - \\
\hline OCWD-KB1 & $10 / 15 / 1998$ & -7.94 & - & - & - & - & - & - & - & - & - \\
\hline OCWD-KB1 & $10 / 22 / 1998$ & -10.03 & - & - & - & - & - & - & - & - & - \\
\hline OCWD-KB1 & $10 / 28 / 1998$ & -11.50 & - & - & - & - & - & - & - & - & - \\
\hline OCWD-KB1 & $11 / 4 / 1998$ & -11.73 & - & - & - & - & - & - & - & - & - \\
\hline OCWD-KB1 & 11/9/1998 & -11.72 & - & - & - & - & - & - & - & - & - \\
\hline OCWD-KB1 & 11/19/1998 & -11.71 & - & - & - & - & - & - & - & - & - \\
\hline OCWD-KB1 & $12 / 7 / 1998$ & -11.72 & - & - & - & - & - & - & - & - & - \\
\hline OCWD-KB1 & $12 / 18 / 1998$ & -9.72 & - & - & - & - & - & - & - & - & - \\
\hline OCWD-KB1 & $12 / 28 / 1998$ & -7.79 & - & - & - & - & - & - & - & - & - \\
\hline OCWD-KB1 & 1/12/1999 & -7.52 & - & - & - & - & - & - & - & - & - \\
\hline OCWD-KB1 & 1/19/1999 & -7.61 & - & - & - & - & - & - & - & - & - \\
\hline OCWD-KB1 & 2/1/1999 & -7.74 & - & - & - & - & - & - & - & - & - \\
\hline $\mathrm{O}-23$ & $4 / 25 / 1995$ & -6.90 & -52 & 32.00 & 28.9 & 160.0 & - & - & 85 & -10.8 & 195 \\
\hline OPWC & $4 / 25 / 1995$ & -6.80 & -55 & 20.00 & - & - & - & - & 85 & -10.8 & 195 \\
\hline OPWC & $1 / 28 / 1996$ & -6.74 & - & - & - & - & - & - & - & - & - \\
\hline OPWC & 2/28/1996 & -6.79 & - & - & - & - & - & - & - & - & - \\
\hline OPWC & $5 / 2 / 1996$ & -6.92 & - & - & - & - & - & - & - & - & - \\
\hline OPWC & $6 / 17 / 1996$ & -6.89 & - & - & - & - & - & - & - & - & - \\
\hline OPWC & 7/17/1996 & -6.95 & - & - & - & - & - & - & - & - & - \\
\hline OPWC & $8 / 29 / 1996$ & -6.74 & - & - & - & - & - & - & - & - & - \\
\hline SAR-6/1 & $5 / 6 / 1996$ & -8.09 & - & - & 7.8 & - & - & - & - & - & - \\
\hline SAR-6/2 & $5 / 6 / 1996$ & -8.01 & - & - & - & - & - & - & - & - & - \\
\hline SAR- $6 / 2$ & $6 / 25 / 1996$ & - & - & - & 10.2 & - & - & - & - & - & - \\
\hline SAR- $6 / 3$ & $5 / 6 / 1996$ & -8.01 & - & - & - & - & - & - & - & - & - \\
\hline SAR-6/4 & $5 / 6 / 1996$ & -8.04 & - & - & - & - & - & - & - & - & - \\
\hline SAR-6/4 & $6 / 25 / 1996$ & - & - & - & 15.3 & - & - & - & - & - & - \\
\hline SAR- $6 / 5$ & $5 / 6 / 1996$ & -8.01 & - & - & - & - & - & - & - & - & - \\
\hline SAR-6/6 & $5 / 6 / 1996$ & -8.04 & - & - & - & - & - & - & - & - & - \\
\hline SAR-6/7 & $5 / 3 / 1996$ & -8.46 & - & - & - & - & - & - & - & - & - \\
\hline SAR-6/8 & $5 / 3 / 1996$ & -8.40 & - & - & - & - & - & - & - & - & - \\
\hline SAR-6/9 & $5 / 3 / 1996$ & -8.40 & - & - & - & - & - & - & - & - & - \\
\hline SAR-6/10 & $5 / 3 / 1996$ & -8.44 & - & - & - & - & - & - & - & - & - \\
\hline
\end{tabular}


Table 1: Preliminary Isotope Data, Ages, and Recharge Temperatures calculated for Groundwater in the Forebay

\begin{tabular}{|c|c|c|c|c|c|c|c|c|c|c|c|}
\hline sample & collection date & $\delta^{18} \mathrm{O}$ & $\delta \mathbf{D}$ & ${ }^{3} \mathrm{H}$ pCi/L & ${ }^{3} \mathrm{H}^{3} \mathrm{He}$ age (yrs) & ${ }^{3} \mathrm{H}$ initial pCi/L & recharge $\mathrm{T}^{\circ} \mathrm{C}$ & excess air $\left(\mathrm{cc} / \mathrm{LH}_{2} \mathrm{O}\right)$ & ${ }^{14} \mathrm{C}$ pmc & $\delta^{13} \mathrm{C}$ per mil & DIC (mg/L HC \\
\hline SAR-7/1 & $1 / 30 / 1996$ & -7.87 & - & - & - & - & - & - & - & - & - \\
\hline SAR-7/1 & $5 / 30 / 1996$ & -7.92 & - & - & - & - & - & - & - & - & - \\
\hline SAR-7/2 & $1 / 30 / 1996$ & -7.91 & - & - & - & - & - & - & - & - & - \\
\hline SAR-7/2 & $5 / 30 / 1996$ & -7.94 & - & - & - & - & - & - & - & - & - \\
\hline SAR-7/2 & $6 / 25 / 1996$ & - & - & - & 12.2 & - & - & - & - & - & - \\
\hline SAR-7/3 & $1 / 30 / 1996$ & -7.93 & - & - & - & - & - & - & - & - & - \\
\hline SAR-7/3 & $5 / 30 / 1996$ & -7.98 & - & - & - & - & - & - & - & - & - \\
\hline SAR-7/4 & $1 / 30 / 1996$ & -7.86 & - & - & - & - & - & - & - & - & - \\
\hline SAR-7/4 & $5 / 30 / 1996$ & -7.97 & - & - & - & - & - & - & - & - & - \\
\hline SAR-7/4 & $6 / 25 / 1996$ & - & - & - & 10.1 & - & - & - & - & - & - \\
\hline SAR-7/5 & $1 / 30 / 1996$ & -7.87 & - & - & - & - & - & - & - & - & - \\
\hline SAR-7/5 & $5 / 30 / 1996$ & -7.75 & - & - & - & - & - & - & - & - & - \\
\hline SAR-7/5 & $6 / 25 / 1996$ & - & - & - & 6.8 & - & - & - & - & - & - \\
\hline SAR-7/6 & $1 / 30 / 1996$ & -7.86 & - & - & - & - & - & - & - & - & - \\
\hline SAR-7/6 & $5 / 30 / 1996$ & -7.85 & - & - & - & - & - & - & - & - & - \\
\hline SAR-7/6 & $6 / 25 / 1996$ & -7.86 & - & - & 7.8 & - & - & - & - & - & - \\
\hline SAR-7/7 & $1 / 30 / 1996$ & -8.23 & - & - & - & - & - & - & - & - & - \\
\hline SAR-7/7 & $5 / 30 / 1996$ & -8.23 & - & - & - & - & - & - & - & - & - \\
\hline SAR-7/7 & $6 / 25 / 1996$ & - & - & - & 8.7 & - & 7 & 0.0158 & - & - & - \\
\hline SAR-7/8 & $1 / 30 / 1996$ & -8.51 & - & - & - & - & - & - & - & - & - \\
\hline SAR-7/8 & $5 / 30 / 1996$ & -8.50 & - & - & - & - & - & - & - & - & - \\
\hline SAR-7/9 & $1 / 30 / 1996$ & -8.36 & - & - & - & - & - & - & - & - & - \\
\hline SAR-7/9 & $5 / 30 / 1996$ & -8.36 & - & - & - & - & - & - & - & - & - \\
\hline SAR-8/2 & - & - & - & - & 17.4 & - & - & - & - & - & - \\
\hline SAR-IMP & $3 / 1 / 1995$ & -7.50 & - & - & - & - & - & - & - & - & - \\
\hline SAR-IMP & 6/1/1995 & -7.70 & - & - & - & - & - & - & - & - & - \\
\hline SAR-IMP & 9/19/1995 & -7.68 & - & - & - & - & - & - & - & - & - \\
\hline SAR-IMP & $10 / 17 / 1995$ & -7.73 & - & - & - & - & - & - & - & - & - \\
\hline SAR-IMP & $11 / 14 / 1995$ & -7.82 & - & - & - & - & - & - & - & - & - \\
\hline SAR-IMP & $12 / 12 / 1995$ & -7.95 & - & - & - & - & - & - & - & - & - \\
\hline SAR-IMP & 1/16/1996 & -7.91 & - & - & - & - & - & - & - & - & - \\
\hline SAR-IMP & 2/20/1996 & -8.03 & - & - & - & - & - & - & - & - & - \\
\hline SAR-IMP & 3/19/1996 & -7.59 & - & - & - & - & - & - & - & - & - \\
\hline SAR-IMP & $4 / 16 / 1996$ & -8.11 & - & - & - & - & - & - & - & - & - \\
\hline SAR-IMP & $5 / 14 / 1996$ & -8.16 & - & - & - & - & - & - & - & - & - \\
\hline SAR-IMP & 6/18/1996 & -8.18 & - & - & - & - & - & - & - & - & - \\
\hline SAR-IMP & $7 / 16 / 1996$ & -8.09 & - & - & - & - & - & - & - & - & - \\
\hline SAR-IMP & $8 / 27 / 1996$ & -7.67 & - & - & - & - & - & - & - & - & - \\
\hline
\end{tabular}


Table 1: Preliminary Isotope Data, Ages, and Recharge Temperatures calculated for Groundwater in the Forebay

\begin{tabular}{|c|c|c|c|c|c|c|c|c|c|c|c|}
\hline sample & collection date & $\delta^{18} \mathbf{O}$ & $\delta \mathbf{D}$ & ${ }^{3} \mathrm{H}$ pCi/L & ${ }^{3} \mathrm{H}-{ }^{3} \mathrm{He}$ age (yrs) & ${ }^{3} \mathrm{H}$ initial pCi/L & recharge $\mathrm{T}^{\circ} \mathrm{C}$ & excess air $\left(\mathrm{cc} / \mathrm{LH}_{2} \mathrm{O}\right)$ & ${ }^{14} \mathrm{C}$ pmc & $\delta^{13} \mathrm{C}$ per mil & DIC (mg/L H \\
\hline SCWC-PLJ2 & $4 / 24 / 1995$ & -10.50 & -87 & 43.00 & 2.1 & 48.0 & - & - & - & -6.3 & 152 \\
\hline SCWC-PLJ2 & $8 / 30 / 1995$ & -10.13 & - & - & - & - & - & - & - & - & - \\
\hline SCWC-PLJ2 & 9/20/1995 & -9.91 & - & - & - & - & - & - & - & - & - \\
\hline SCWC-PLJ2 & $10 / 25 / 1995$ & -9.41 & - & - & - & - & - & - & - & - & - \\
\hline SCWC-PLJ2 & $11 / 20 / 1995$ & -9.03 & - & - & - & - & - & - & - & - & - \\
\hline SCWC-PLJ2 & $12 / 1 / 1995$ & -8.92 & - & - & - & - & - & - & - & - & - \\
\hline SCWC-PLJ2 & $1 / 19 / 1996$ & -8.65 & - & - & - & - & - & - & - & - & - \\
\hline SCWC-PLJ2 & $5 / 2 / 1996$ & -7.91 & - & - & - & - & - & - & - & - & - \\
\hline SCWC-PLJ2 & $5 / 15 / 1996$ & -7.80 & - & - & - & - & - & - & - & - & - \\
\hline SCWC-PLJ2 & $6 / 17 / 1996$ & -7.67 & - & - & - & - & - & - & - & - & - \\
\hline SCWC-PLJ2 & $7 / 31 / 1996$ & - & - & - & 3.1 & - & 22 & 0.0126 & - & - & - \\
\hline SCWC-PLJ2 & $5 / 28 / 1996$ & -7.76 & - & - & 2.6 & - & 23 & 0.0125 & - & - & - \\
\hline SCWC-PLJ2 & 2/13/1997 & -7.53 & - & - & - & - & - & - & - & - & - \\
\hline SCWC-PLJ2 & $4 / 28 / 1997$ & -7.54 & - & - & - & - & - & - & - & - & - \\
\hline SCWC-PLJ2 & $7 / 17 / 1997$ & -7.72 & - & - & - & - & - & - & - & - & - \\
\hline SCWC-PLJ2 & $8 / 29 / 1997$ & -7.59 & - & - & - & - & - & - & - & - & - \\
\hline SCWC-PLJ2 & $10 / 22 / 1997$ & -7.55 & - & - & - & - & - & - & - & - & - \\
\hline SCWC-PLJ2 & $12 / 19 / 1997$ & -7.61 & - & - & - & - & - & - & - & - & - \\
\hline SCWC-PBF3 & 9/27/1996 & -9.01 & - & - & - & - & - & - & - & - & - \\
\hline SCWC-PBF3 & $12 / 19 / 1997$ & -8.95 & - & - & - & - & - & - & - & - & - \\
\hline SCWC-PBF4 & $5 / 23 / 1996$ & -8.56 & - & - & 27.9 & - & 19 & 0.0141 & - & - & - \\
\hline SCWC-PBF4 & 9/27/1996 & -8.47 & - & - & - & - & 18 & 0.0176 & - & - & - \\
\hline SCWC-PBF4 & $11 / 14 / 1997$ & -8.15 & - & - & - & - & - & - & - & - & - \\
\hline SCWC-PBF4 & $12 / 19 / 1997$ & -8.27 & - & - & - & - & - & - & - & - & - \\
\hline Santiago Basin & 6/1/1995 & -6.30 & - & - & - & - & - & - & - & - & - \\
\hline Santiago Basin & $8 / 30 / 1995$ & -6.24 & - & - & - & - & - & - & - & - & - \\
\hline Santiago Basin & 9/21/1995 & -6.48 & - & - & - & - & - & - & - & - & - \\
\hline Santiago Basin & $11 / 17 / 1995$ & -6.53 & - & - & - & - & - & - & - & - & - \\
\hline Santiago Basin & $12 / 14 / 1995$ & -6.49 & - & - & - & - & - & - & - & - & - \\
\hline Santiago Basin & $1 / 24 / 1996$ & -6.70 & - & - & - & - & - & - & - & - & - \\
\hline Santiago Basin & 2/28/1996 & -6.90 & - & - & - & - & - & - & - & - & - \\
\hline Santiago Basin & $3 / 28 / 1996$ & -7.09 & - & - & - & - & - & - & - & - & - \\
\hline Santiago Basin & $5 / 2 / 1996$ & -6.61 & - & - & - & - & - & - & - & - & - \\
\hline Santiago Basin & $6 / 17 / 1996$ & -5.44 & - & - & - & - & - & - & - & - & - \\
\hline Santiago Basin & 7/17/1996 & -5.55 & - & - & - & - & - & - & - & - & - \\
\hline Santiago Basin & $8 / 27 / 1996$ & -5.57 & - & - & - & - & - & - & - & - & - \\
\hline SID-3 & $4 / 25 / 1995$ & -7.20 & -52 & 23.00 & 8.1 & 33.0 & 18 & 0.0223 & 101 & -12.5 & 227 \\
\hline SID-3 & $8 / 30 / 1995$ & -7.24 & - & - & - & - & 16 & 0.0143 & - & - & - \\
\hline SID-4 & 3/9/1995 & - & - & - & 4.1 & - & - & - & - & - & - \\
\hline SID-4 & 9/21/1995 & -6.35 & - & - & - & - & - & - & - & - & - \\
\hline SID-4 & $1 / 24 / 1996$ & -6.56 & - & - & - & - & - & - & - & - & - \\
\hline SID-4 & $3 / 28 / 1996$ & -6.60 & - & - & - & - & - & - & - & - & - \\
\hline
\end{tabular}


Table 1: Preliminary Isotope Data, Ages, and Recharge Temperatures calculated for Groundwater in the Forebay

\begin{tabular}{|c|c|c|c|c|c|c|c|c|c|c|c|}
\hline sample & collection date & $\delta^{18} \mathrm{O}$ & $\delta \mathbf{D}$ & ${ }^{3} \mathrm{H}$ pCi/L & ${ }^{3} \mathrm{H}-{ }^{3} \mathrm{He}$ age (yrs) & ${ }^{3} \mathrm{H}$ initial pCi/L & recharge $\mathrm{T}^{\circ} \mathrm{C}$ & excess air $\left(\mathrm{cc} / \mathrm{LH}_{2} \mathrm{O}\right)$ & ${ }^{14} \mathrm{C} \mathrm{pmc}$ & $\delta^{13} \mathrm{C}$ per mil & DIC (mg/L $\left.\mathrm{HCO}_{3}\right)$ \\
\hline SID-4 & $5 / 2 / 1996$ & -6.50 & - & - & - & - & - & - & - & - & - \\
\hline SID-4 & $6 / 17 / 1996$ & -6.56 & - & - & - & - & - & - & - & - & - \\
\hline SID-4 & 9/30/1996 & -6.36 & - & - & - & - & - & - & - & - & - \\
\hline WBS-2A/2 & - & - & - & - & 3.3 & - & - & - & - & - & - \\
\hline WBS2-A/3 & - & - & - & - & 8.0 & - & - & - & - & - & - \\
\hline WBS-3/2 & - & - & - & - & 2.1 & - & - & - & - & - & - \\
\hline WBS-4/2 & - & - & - & - & 19.0 & - & - & - & - & - & - \\
\hline WBS- $4 / 3$ & - & - & - & - & 0.7 & - & - & - & - & - & - \\
\hline YLWD-5 & $6 / 21 / 1995$ & -8.00 & -59 & 34.00 & 11.4 & 64.0 & 15 & 0.0061 & - & - & - \\
\hline YLWD-5 & $7 / 23 / 1996$ & -7.82 & - & - & - & - & - & - & - & - & - \\
\hline YLWD-11 & 3/9/1995 & -8.00 & -59 & 22.00 & 3.9 & 27.0 & 13 & 0.0092 & 103 & -11.3 & 226 \\
\hline YLWD-11 & $6 / 21 / 1995$ & -8.00 & -57 & 23.00 & 3.6 & 28.0 & 15 & 0.0095 & - & - & - \\
\hline YLWD-11 & $9 / 21 / 1995$ & -7.81 & - & - & - & - & - & - & - & - & - \\
\hline YLWD-11 & $10 / 18 / 1995$ & -7.72 & - & - & - & - & - & - & - & - & - \\
\hline YLWD-11 & $11 / 15 / 1995$ & -7.73 & - & - & - & - & - & - & - & - & - \\
\hline YLWD-11 & $12 / 11 / 1995$ & -7.75 & - & - & - & - & - & - & - & - & - \\
\hline YLWD-11 & $1 / 22 / 1996$ & -7.78 & - & - & - & - & - & - & - & - & - \\
\hline YLWD-11 & $5 / 16 / 1996$ & -7.78 & - & - & - & - & - & - & - & - & - \\
\hline YLWD-11 & 7/17/1996 & -7.78 & - & - & - & - & 17 & 0.0084 & - & - & - \\
\hline YLWD-11 & $5 / 28 / 1996$ & -7.97 & - & - & 3.0 & - & 16 & 0.0119 & - & - & - \\
\hline YLWD-11 & $6 / 20 / 1996$ & -7.75 & - & - & - & - & - & - & - & - & - \\
\hline YLWD-11 & $8 / 27 / 1996$ & -7.83 & - & - & - & - & - & - & - & - & - \\
\hline YLWD-15 & $6 / 20 / 1995$ & -8.00 & -61 & 28.00 & 0.0 & - & 14 & 0.0093 & - & - & 225 \\
\hline YLWD-15 & $5 / 28 / 1996$ & - & - & - & 1.5 & - & 16 & 0.011 & - & - & - \\
\hline
\end{tabular}




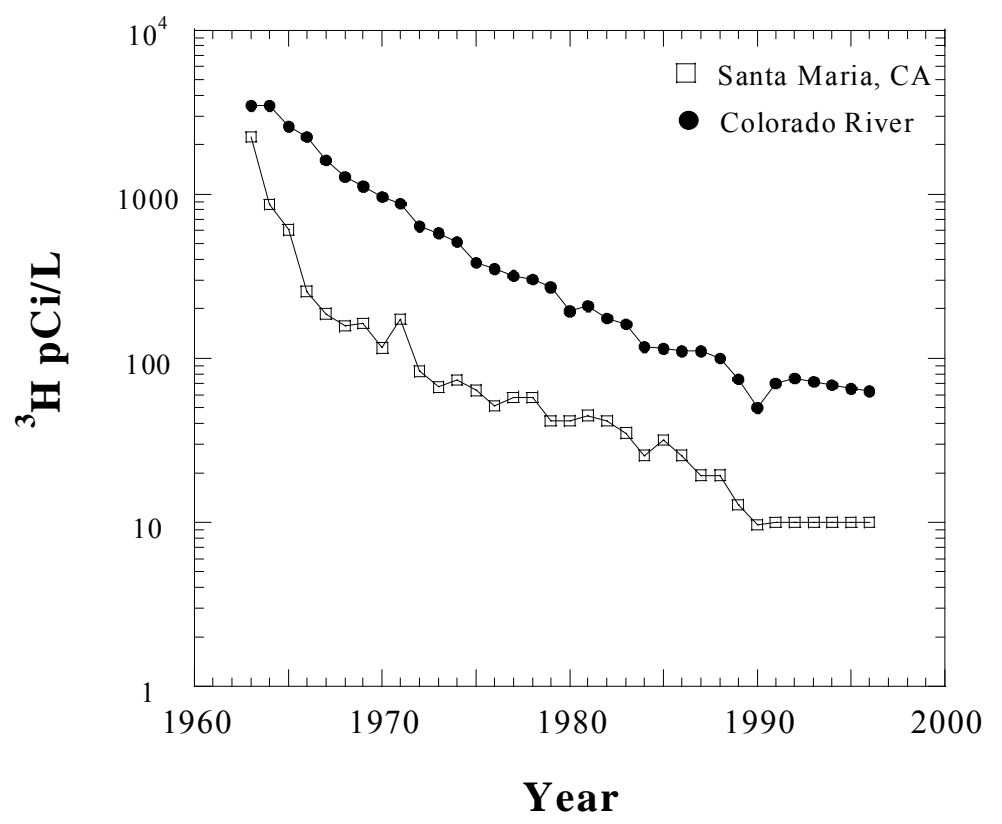

Figure 3. Comparison of ${ }^{3} \mathrm{H}$ concentration in Colorado River water measured above Cisco, Utah and precipitation collected along the coast in Santa Maria California. Note that the coastal precipitation had nearly five times lower ${ }^{3} \mathrm{H}$ than in the Colorado River for the same year.

\subsubsection{Noble Gas Results}

Tritium decays to ${ }^{3} \mathrm{He}$ at a constant rate with a half-life of 12.43 years. As such, the relative abundance between these two constituents is proportional to time. Once ${ }^{3} \mathrm{H}$ is recharged with groundwater and is isolated from the atmosphere, its decay to ${ }^{3} \mathrm{He}$ behaves like a simple chronometer. However, during recharge ${ }^{3} \mathrm{He}$ from the atmosphere is also incorporated in the groundwater and must be factored in.

The calculation of ${ }^{3} \mathrm{H}^{-3} \mathrm{He}$ ages for the Forebay groundwater required independent measurement of dissolved noble gas concentrations. The minimum required data for the age calculation are the dissolved ${ }^{3} \mathrm{H},{ }^{3} \mathrm{He},{ }^{4} \mathrm{He}$, and Ne concentrations (Schlosser et al., 1988; Poreda et al., 1988). Neon dissolved in shallow groundwater originates exclusively from the atmosphere, and its measured concentration is an indicator of the amount of dissolved air in groundwater. This component is necessary in order to distinguish atmospheric ${ }^{3} \mathrm{He}$ in the groundwater from ${ }^{3} \mathrm{He}$ derived from ${ }^{3} \mathrm{H}$ decay.

For this study $\mathrm{Ar}, \mathrm{Kr}$, and $\mathrm{Xe}$ were also for each groundwater collected in the Forebay. The relative abundance among all the noble gases provided a more accurate means to quantify gas derived from equilibrium solubility and to separate it from dissolved gas derived from non-equilibrium entrainment of the atmosphere. This latter component is known as "excess air" (Stute and Schlosser, 1993; Aeschbach-Hertig, et al., 1999), and is commonly observed in groundwater (Heaton and Vogel, 1981). The atmospheric component to the ${ }^{3} \mathrm{H}-{ }^{3} \mathrm{He}$ ages is calculated by iterative subtraction of excess air contributions using each noble gas concentration until recharge temperatures and excess air concentrations are in agreement among each noble gas species. Any 
inconsistencies arising from this approach is usually due to compromised samples, or from a recharge process where incomplete dissolution of excess air results.

Calculated recharge temperatures ranged between 7 and $34^{\circ} \mathrm{C}$. The average recharge temperature among all samples measured was $19.8^{\circ} \mathrm{C} \pm 5.7$, which is similar to the mean temperature of the surface water in the SAR watershed derived from $>600$ measurements from 1992 to 1999 (mean $=21.0^{\circ} \mathrm{C} \pm 5.0$ ). The highest recharge temperature $\left(34^{\circ} \mathrm{C}\right)$ was calculated for AMD-9 level 1 collected in September, 1996. As will be discussed in section 8, groundwater at AMD-9 level one originates from Anaheim Lake recharge water with a travel time of less than one month, and the high recharge temperature is likely consistent with mid-summer shallow water temperatures in the lake.

As a simple point of reference, Table 2 presents concentrations for noble gases dissolved at equilibrium in water at $35^{\circ} \mathrm{C}$ and at sea level. In the Forebay, the measured temperature of SAR recharge water typically falls into a range between $11^{\circ} \mathrm{C}$ for the wintertime and $35^{\circ} \mathrm{C}$ for the summer time (Fig. 4). Therefore, a groundwater that recharged into the Forebay free of any excess air component should have dissolved noble gas concentrations approximately equal to or greater than (lower temperature) those presented for conditions in Table 2. All noble gas concentrations measured in groundwater samples collected in the Forebay exceed concentrations listed in Table 2, consistent with recharge temperatures $<35^{\circ} \mathrm{C}$ and/or incorporation of excess air.

\section{Table 2: Equilibrium Solubility of Noble Gases in Water at $35^{\circ} \mathrm{C}$ and at Sea Level.}

\begin{tabular}{lc}
$\begin{array}{l}\text { Noble } \\
\text { Gas }\end{array}$ & $\begin{array}{c}\text { Equilibrium } \\
\text { Solubility }\left(\mathrm{cc} / \mathrm{g} \mathrm{H}_{2}\right.\end{array}$ \\
\hline $\mathrm{He}$ & $4.5 \times 10^{-8}$ \\
$\mathrm{Ne}$ & $1.8 \times 10^{-7}$ \\
$\mathrm{Ar}$ & $2.5 \times 10^{-4}$ \\
$\mathrm{Kr}$ & $5.1 \times 10^{-8}$ \\
$\mathrm{Xe}$ & $6.7 \times 10^{-9}$
\end{tabular}

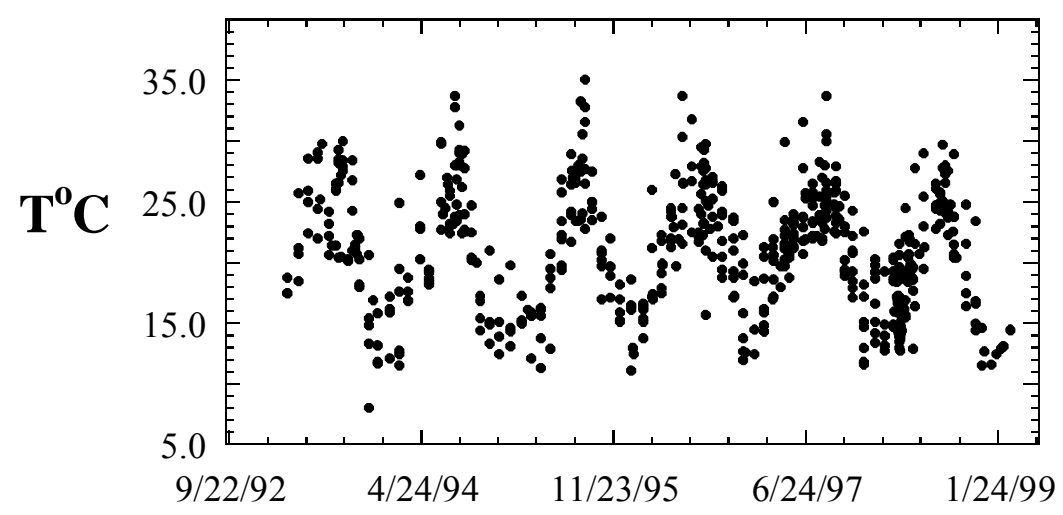

Figure 4. Variation in surface water temperature measured in the SAR from 1993 to 1999 showing a range from approximately $10-35^{\circ} \mathrm{C}$ from winter to summer. 
Calculated dissolved excess air concentrations ranged from $<1 \times 10^{-3}$ to $\sim 7.5 \times 10^{-2} \mathrm{cc}$ per gram of water. Most concentrations were around $1 \times 10^{-2} \mathrm{cc} / \mathrm{g} \mathrm{H}_{2} \mathrm{O}$, consistent with previous observations in alluvial groundwater systems (e.g. Stute et al., 1997; Shapiro et al., 1998). The total measured helium gas dissolved in Forebay groundwater was 30 to $470 \%$ in excess over the calculated equilibrium concentration (determined by $\mathrm{Ne}, \mathrm{Ar}, \mathrm{Kr}$, and $\mathrm{Xe}$ ). However, in the majority of groundwater samples, the helium excess was due to excess air. This is best illustrated by a plot of relative $\mathrm{Ne}$ against relative ${ }^{4} \mathrm{He}$ concentrations and comparing them to concentrations expected for equilibrium and dissolved excess air contribution (Fig. 5). Deviations from predicted equilibrium+excess air contribution would indicate radiogenic ${ }^{4} \mathrm{He}$ accumulation. Only a few groundwater samples fall into this latter category. Wells with radiogenic ${ }^{4} \mathrm{He}$ are AMD-6 levels 10 and 13, AMD-7 level 13, SAR-1, SAR-2, and F-AIRP. These samples represent deeper collection points or distal regions of main flowpaths in the Forebay (see below).

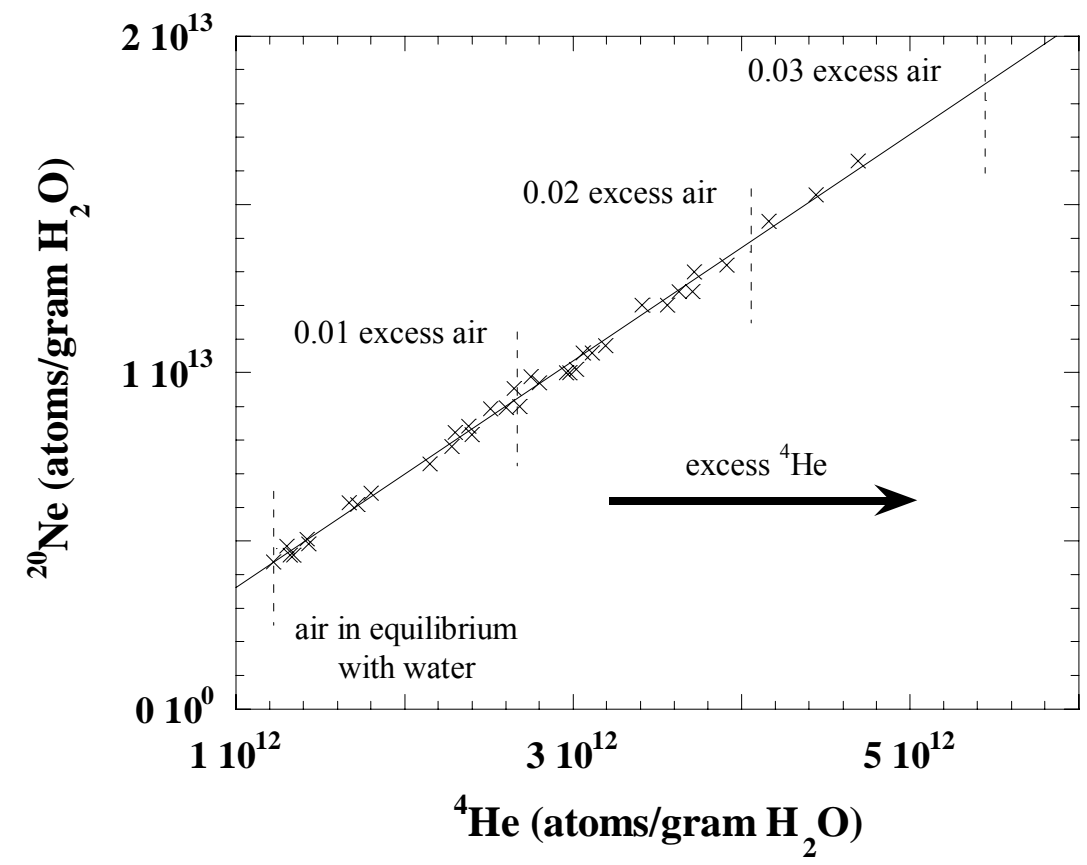

Figure 5. The linear correspondence of $\mathrm{Ne}$ to ${ }^{4} \mathrm{He}$ abundance and adherence to excess air concentration models indicates little if any radiogenc ${ }^{4} \mathrm{He}$ is dissolved in most samples analyzed in the Forebay groundwater. Exceptions are background wells FAIRP, A-47, and some deep zones sampled in Westbay wells.

Radiogenic ${ }^{4} \mathrm{He}$ is also illustrated by the ${ }^{3} \mathrm{He} /{ }^{4} \mathrm{He}$ ratio normalized to air (see Davisson et al., 1999). When this ratio is $<1$, it indicates accumulation of radiogenic helium, whereas a ratio $>1$ indicates groundwater having tritiogenic ${ }^{3} \mathrm{He}$ from ${ }^{3} \mathrm{H}$ decay. A ratio that equals one indicates groundwater $<1$ year old, since no radiogenic ${ }^{4} \mathrm{He}$ or tritiogenic ${ }^{3} \mathrm{He}$ has accumulated.

Groundwater sampled on three separate occasions from well AMD-9 level 1 in September and October 1996. Its calculated recharge temperature approached $35^{\circ} \mathrm{C}$ for the three separate water samples, and its calculated excess air was the lowest observed of 
all wells. Similarly in well A-27, the calculated recharge temperature ranged from $12^{\circ} \mathrm{C}$ in April 1995 to $32^{\circ} \mathrm{C}$ in October 1996, consistent with seasonal air temperature variation and a one month old age for groundwater in this well (see below).

The lowest recharge temperature was $7^{\circ} \mathrm{C}$ calculated for well SAR-7 level 7. A similarly low recharge temperature $\left(8^{\circ} \mathrm{C}\right)$ was calculated for well AMD-1 level 8 . Such low recharge temperatures are at the extreme of surface water temperature measurements, and are slightly lower than mean annual minimum air temperature $\left(9.7^{\circ} \mathrm{C}\right.$ in Yorba Linda). There is no indication that the samples were compromised during sampling, and furthermore, loss of sample integrity should result in poor recharge temperature agreement among the noble gases, which was not the case for these particular samples. Monthly minimum temperature can be as low as $5.4^{\circ} \mathrm{C}$ in January near the recharge area, suggesting this groundwater was recharged during winter months.

\subsubsection{Calculated Ages}

The relatively low or undetectable radiogenic ${ }^{4} \mathrm{He}$ observed in most Forebay groundwater samples eliminates the need to subtract this component from the measured helium and simplifies the groundwater age calculation. Calculated ages range from less than one to 31 years (Fig. 6). Where ${ }^{3} \mathrm{H}$ was either non-detectable or at appreciably low concentration $(\leq 5 \mathrm{pCi} / \mathrm{L})$, an age greater than 40 years was assigned. These particular samples also have radiogenic ${ }^{4} \mathrm{He}$. The greatest uncertainty in the calculated age occurs for groundwater samples having a young age and relatively high excess air concentration, since a large excess air ${ }^{3} \mathrm{He}$ contribution was subtracted from a small amount of tritiogenic ${ }^{3} \mathrm{He}$.

Adding together the measured ${ }^{3} \mathrm{H}$ concentration with the tritiogenic ${ }^{3} \mathrm{He}$ yields a ${ }^{3} \mathrm{H}$ concentration in the groundwater at the time of recharge, referred to as the initial ${ }^{3} \mathrm{H}$. The calculation of this initial ${ }^{3} \mathrm{H}$ is important in order to validate the age-dating measurements and calculations by demonstrating the increase in initial ${ }^{3} \mathrm{H}$ with backward progression in time (see Fig. 7). Furthermore, the reconstruction of initial ${ }^{3} \mathrm{H}$ from the measured groundwater samples provides a basis to calculate the ${ }^{3} \mathrm{H}$ inventory in groundwater and compare it to the ${ }^{3} \mathrm{H}$ concentration of recharge water over time. From Figure 7 it is shown that the calculated initial ${ }^{3} \mathrm{H}$ increased in recharge water backward in time, and the highest initial ${ }^{3} \mathrm{H}$ concentration was $678 \mathrm{pCi} / \mathrm{L}$ calculated for well AMD-7 level 10, whose implied recharge was in 1966. It is interesting to compare this concentration to ${ }^{3} \mathrm{H}$ concentration in precipitation at Santa Maria, CA and the Colorado River in 1966. The ${ }^{3} \mathrm{H}$ measured and weighted to Santa Maria precipitation was $256 \mathrm{pCi} / \mathrm{L}$ (IAEA/WMO, 2001), whereas the Colorado River measured above Cisco, Utah was $2240 \mathrm{pCi} / \mathrm{L}$ (Michel, 1992). As shown later, the $\delta^{18} \mathrm{O}$ value of groundwater in AMD-7 level 10 indicates that a Colorado River source comprised greater than $60 \%$ of this water. Sixty percent of a groundwater with a ${ }^{3} \mathrm{H}$ concentration of $2240 \mathrm{pCi} / \mathrm{L}$ should have a ${ }^{3} \mathrm{H}$ concentration of at least $1340 \mathrm{pCi} / \mathrm{L}$. This concentration is much greater than calculated for an initial ${ }^{3} \mathrm{H}$ in AMD-7 level 10, and suggests that either the fallout ${ }^{3} \mathrm{H}$ data is not an accurate record of recharge ${ }^{3} \mathrm{H}$ concentrations in the Forebay, or ${ }^{3} \mathrm{H}$ and/or ${ }^{3} \mathrm{He}$ was lost from the groundwater systems at some point. Both these possibilities will be discussed later in section 10 . 


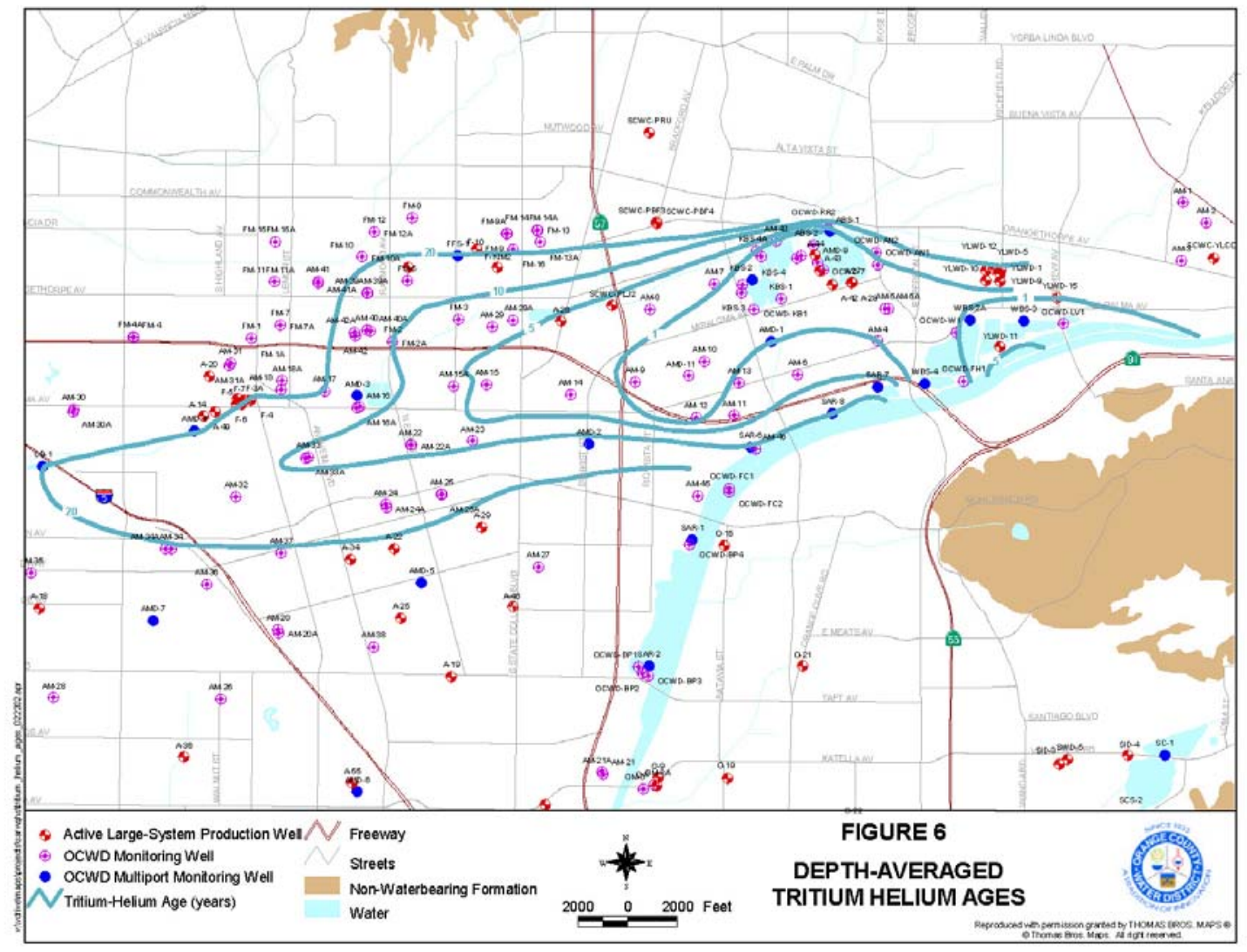

Figure 6. Contoured ${ }^{3} \mathrm{H}-{ }^{3} \mathrm{He}$ ages in the Forebay groundwater show a regular increase with downgradient distance from Anaheim Lake and Kraemer Basin. Age distribution is somewhat more complicated below the SAR. For multiport wells, an average value was plotted, which was calculated for each well using data on only the depths measured.

Groundwater ages measured in the Forebay showed a regular increase from $<1$ to $>20$ years over a four to five mile distance downgradient from the recharge basins and the SAR channel (Fig. 6). The age distribution suggests a west-southwest flow path originating from Anaheim/Kraemer recharge basins stretching toward the Fullerton well field area. Groundwater ages along this flowpath are less than five years old two miles downgradient. The direction of this flow path is consistent with the flow direction deduced by pieziometric levels in wells for this area.

Groundwater in wells ABS-2, SCWC-PBF3, and SCWC-PBF4, a short distance north and northwest of Anaheim Lake and Kraemer Basin, range from 5 to 25 years old, suggesting ages rapidly increased in a direction north of the main groundwater flowpaths originating from Anaheim Lake and Kraemer Basin.

Groundwater has a somewhat older age in the area south of Anaheim Lake and Kraemer Basin and north of the SAR channel. For example, wells AM-13, AM-11, AM6, SAR-6, SAR-7, and SAR-8 ranged in age between 1 and 10 years even though the groundwater zone they occupy appears to be along main recharge and flow paths from the SAR. Wells SAR-6, SAR-7, and SAR-8 were sampled at multiple depths. In the case of SAR-7, groundwater ages were younger in levels 5, 6, and 7 than in level 4 above them. In the second level of SAR- 6 groundwater age was approximately 10 years, and 
increased to 15 years at the fourth level and greater than 40 years at level 7 . These ages are surprisingly old for their depths and their close proximity to the SAR channel.

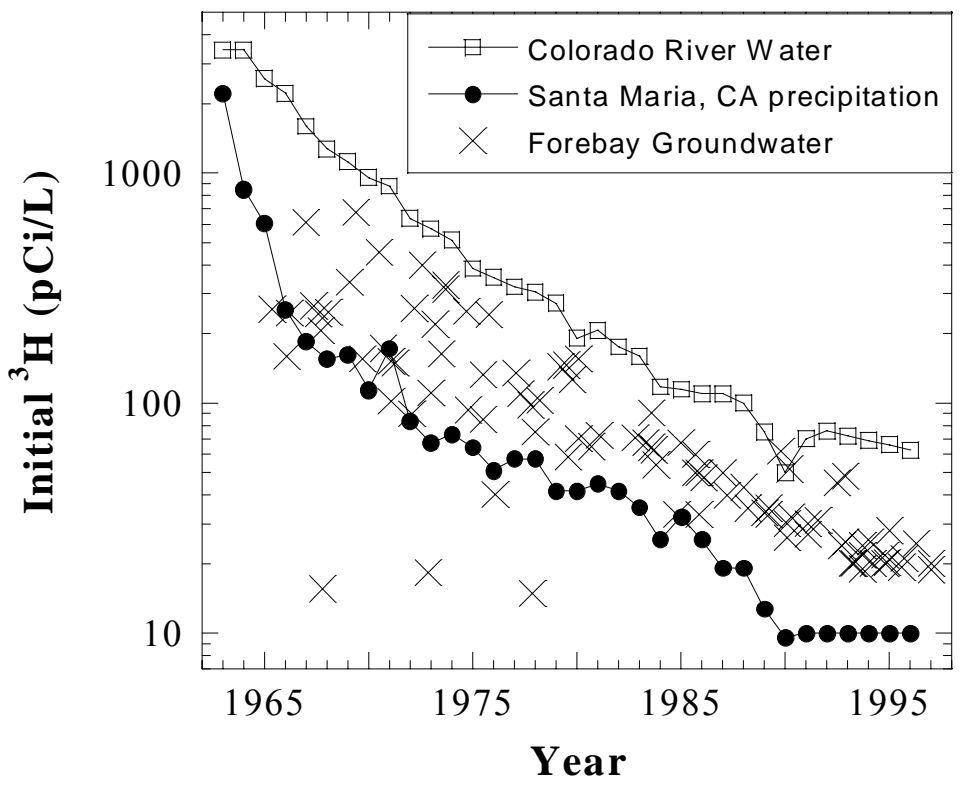

Figure 7. The initial ${ }^{3} \mathrm{H}$ concentration, calculated for wells where ${ }^{3} \mathrm{H}-{ }^{3} \mathrm{He}$ ages were measured, increases with groundwater age or recharge year, as expected for a ${ }^{3} \mathrm{H}$ atmospheric fallout source term. Note that most initial ${ }^{3} \mathrm{H}$ concentrations are intermediate between those measured for the Colorado River and Santa Maria, CA precipitation.

Well HG- ${ }^{1}$, which is located approximately 50 feet from the Imperial headgates and at approximately 50 feet in depth had a measurable groundwater age of approximately one year, even though the water level in this well responds rapidly to river discharge. Note also, the calculated recharge temperature in March, 1995 was $24^{\circ} \mathrm{C}$, consistent with a more summertime value, whereas in June, 1995 the calculated recharge temperature was lower at $19^{\circ} \mathrm{C}$. This relationship may suggest an approximately 6-month travel time to HG-1.

Production well YLWD-11 located adjacent to the Warner and Conrock recharge basins had a groundwater age of approximately 4 years. Well YLWD-5 is over a halfmile from the SAR (believed to be its recharge source) and is perforated from 90-340 feet below the ground surface. It had a mean age of 11 years. YLWD-15, which is perforated fromn 133 to 198 feet below ground surface, is within 1,000 feet of the SAR and had a mean age of $<1$ year.

The wide variation in ${ }^{3} \mathrm{H}-{ }^{3} \mathrm{He}$ ages measured in wells adjacent to the SAR indicates that groundwater recharge and flow from the river toward the Forebay is complex. The complexity is caused by the heterogeneous nature of sediments. The ages suggest that

\footnotetext{
${ }^{1}$ Note that this well has been subsequently damaged beyond repair and no longer represents a viable monitoring point.
} 
there is apparently a relatively significant amount of groundwater storage beneath the river channel and fast-track flowpaths are discrete and difficult to isolate.

Measured ages for groundwater in wells adjacent to Anaheim Lake and Kraemer Basin ranged from $<1$ to 18 years. OCWD-KB1 is located at a shallow depth (180-200 feet below ground surface) adjacent to Kraemer Basin and had a measured age of near-zero. Wells A-27 and AMD-9 level 1 had near-zero ages, while wells of comparable depths like ABS-2 and A-28 had ages 5 and 3 years, respectively. In contrast, the deep wells A42, A-43, and A-44, which are perforated below a low permeability layer at approximately 400 feet below the surface, had mean ages of 18, 12, and 11 years, respectively. Determining the extent to which Anaheim Lake water transports across this low-permeability zone was one of the central questions regarding these particular water supply wells. The increased age with depth below Anaheim Lake is also defined by depth-specific ages in AMD-9, which are near-zero at the upper level (200-220 ft bgs), 3 years old at the second level (450-470 ft bgs), and 25 years old at the fourth level (896$916 \mathrm{ft}$ bgs).

Depth-specific samples were collected for production wells A-42, A-43, and A-44 in February, 1997 during down-hole spinner log tests conducted to determine the depths of preferential groundwater production (West, 1997). Isotope samples were collected while the pump was running by inserting a sampling tube down the well casing and collecting at discrete intervals. Assuming that all flow from the perforations into the casing was upward, each sample interval collected represented an integration of groundwater at that respective sampling depth plus flow from deeper depths. The ages in the depth-specific groundwater samples varied from 12 to 29 years (Fig. 8). Samples for age-dating from well A-43 were not collected successfully and were compromised by air. The groundwater ages in well A-42 showed a narrow range of 17 to 26 years with the youngest actually occurring at the deepest depth, while groundwater ages in well A-44 showed a systematic increase with depth increasing from 12 to 29 years. Note that the age of shallow groundwater in well A-44 (12 years) is similar to the age measured on the total discharge from this well (11 years). This result is consistent with spinner log results, which indicated that in A-44, a larger percentage of the water was produced from the shallow perforation interval compared to A-42 and A-43 (West, 1997). In A-42, the upper zone (430-660 bgs) produced 31 percent of discharge; in A-43, the upper zone (530-620 bgs) produced 32 percent of the discharge; in A-44, the upper zone (450-660 bgs) produced 67 percent of the total discharge (West, 1997). 

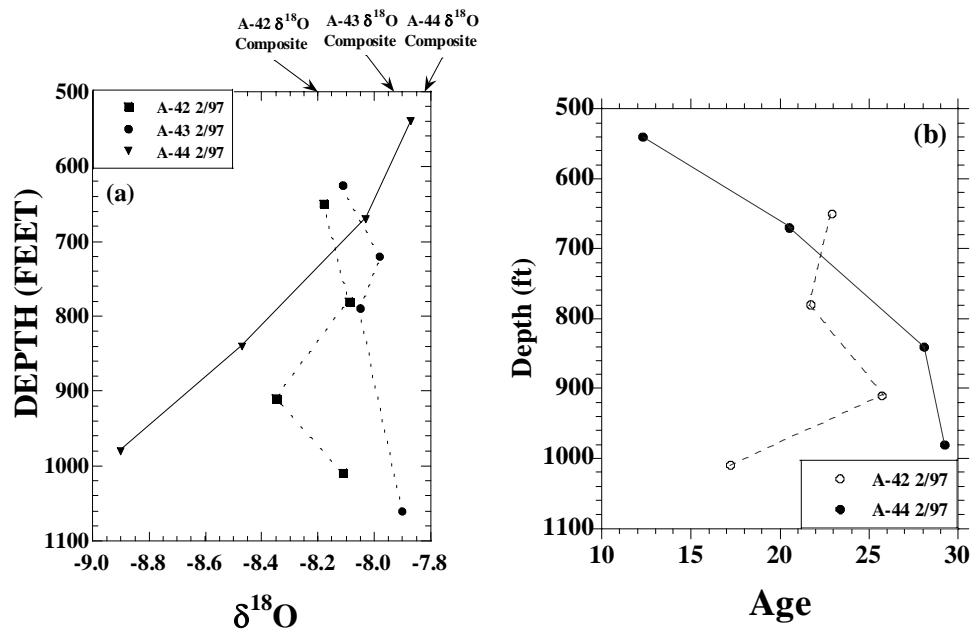

Figure 8. Depth-specific data reveal that the ${ }^{3} \mathrm{H}-{ }^{3} \mathrm{He}$ ages and $\delta^{18} \mathrm{O}$ values in wells A-42 and A-43 are not dramatically different, while well A-44 shows a systematic increase in $\delta^{18} \mathrm{O}$ and a corresponding decrease in age with decreasing depth.

Wells AM-10, AM-9, and AM-14, which are sequentially downgradient of each other (Fig. 7) had measured ages 0,1 , and 2 years, respectively. Similarly, wells AM-7, AM-8, and SCWC-PLJ2 are also sequentially downgradient of each other and had ages of 2, 2, and 3 years, respectively. All of these wells produce groundwater at depths $<500$ feet below the surface.

Groundwater ages of greater than 40 years were observed at deep levels $(>1000 \mathrm{ft}$ bgs) of monitoring wells AMD-5, AMD-6, and AMD-7. These three wells are located south of the primary flow path leading west from Anaheim/Kraemer recharge basins (Fig. 7). Monitoring wells west of this preferred flow path (e.g AMD-2, AMD-3, AMD-4, CB1) sampled and measured at similar depths all had detectable ${ }^{3} \mathrm{H}$ and calculated ages $<40$ years old, even though in the case of AMD-4 level 10, and CB-1 level 6, the ${ }^{3} \mathrm{H}$ content was somewhat low, suggesting mixtures with a non-tritiated water. Groundwater $>40$ years old in deep levels of AMD-5, AMD-6, and AMD-7 also are indicated by a measurable radiogenic ${ }^{4} \mathrm{He}$ content, as revealed by their ${ }^{3} \mathrm{He} /{ }^{4} \mathrm{He}$ ratios of less than one. A similarly low ratio was observed for well F-AIRP located on the northwest end of the study area. Even though F-AIRP has some measurable ${ }^{3} \mathrm{H}$, the low ${ }^{3} \mathrm{He} /{ }^{4} \mathrm{He}$ ratio indicates mixtures of groundwater $>40$ years old. In deep levels of AMD-2, AMD-3, AMD-4, and CB-1, the ${ }^{3} \mathrm{He} /{ }^{4} \mathrm{He}$ ratios are all greater than or equal to one.

Early in the groundwater dating study, additional measurements were performed on wells downgradient of Santiago Pit (Fig. 2a). In these wells, groundwater ages ranged from 5 to 29 years old. Well SID-4 had the youngest age and is located within 500 feet of Santiago Pit. SID-3 is located approximately 2000 feet west and had a groundwater age of 9 years. Well O-23 and EOCW-E are located approximately 1000 and 2000 feet downgradient, respectively, and produced groundwater with ages of 29 and 24 years, respectively. These old groundwater ages at close proximity to the Santiago Pit suggest that a considerable amount of older tritiated groundwater occurs in this area. However, all these wells had ${ }^{3} \mathrm{He} /{ }^{4} \mathrm{He}$ ratios $>1$, indicating groundwater of considerably older ages $\left(>1000\right.$ years) having radiogenic ${ }^{4} \mathrm{He}$ was not detected. 


\subsection{Stable Isotope Results}

The abundance of ${ }^{18} \mathrm{O}$ and deuterium in coastal precipitation varies with inland distance and elevation (Dansgaard, 1964). This effect is persistent in all types of precipitation and at various latitudes along the Pacific coast (Younge et al., 1989; Ingraham and Taylor, 1991). In southern California, the $\delta^{18} \mathrm{O}$ value of local surface water varies from around -6.0 per mil along the coast to less than -12.5 per mil for runoff originating approximately 70 miles inland (Williams and Rodoni, 1997). A similarly large range in $\delta^{18} \mathrm{O}$ is also observed in surface and groundwater of the Orange County basin (Williams, 1997). This is due to runoff in the SAR originating as drainage from high inland elevations. In addition, imported Colorado River water, which is derived from the Colorado Rocky Mountains, has even lower $\delta \mathrm{D}$ and $\delta^{18} \mathrm{O}$ values.

Previous measurements in the Orange County basin has shown that surface water and groundwater can be delineated into four general groups based on their stable isotope composition (Williams, 1994; Williams, 1997). These four groups are categorized as 1) "local recharge" originating as local precipitation and surface runoff in the Chino Hills and Santa Ana Mts., 2) "native recharge" originating from pre-development SAR infiltration, 3) "recent recharge" originating from modern SAR infiltration, and 4) "Colorado River mixtures" observed in groundwater due to the historical use of this imported source to supplement recharge (Fig. 9).

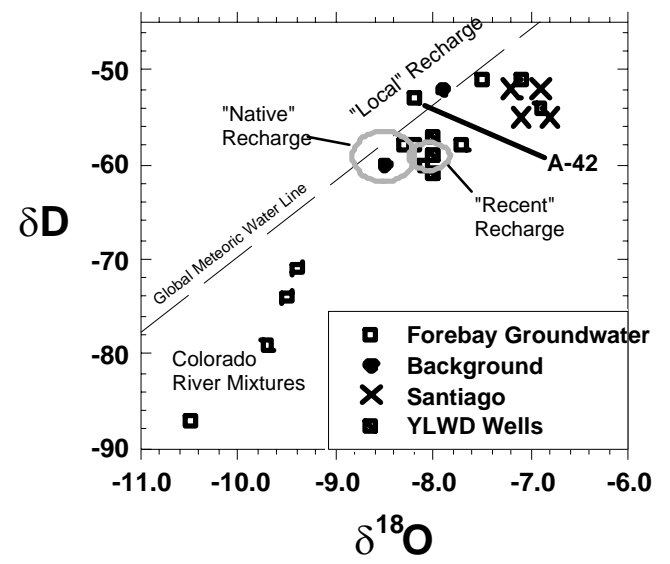

Figure 9. The $\delta \mathrm{D}-\delta^{18} \mathrm{O}$ data of Forebay groundwater generally lie within spatial regions defined by Williams (1997). Groundwater associated with Santiago Pit recharge tends to have slightly higher values, reflecting incorporation of more locally derived recharge.

These previous studies using the stable isotope composition of recharge water in the Orange County basin lacked repeat sample analysis. Given that the District recharges greater than 200,000 acre-ft per year in an area of less than $10 \mathrm{mi}^{2}$, large changes in the stable isotope composition of groundwater near and in the recharge areas of the Forebay is expected. Therefore, LLNL began periodic sampling of surface water and groundwater from several wells in the Forebay and measured them for $\delta^{18} \mathrm{O}$, with the purpose of documenting changes in the isotope signature related to changes in recharge source. The success of this approach depended on temporal variability in the $\delta^{18} \mathrm{O}$ of the recharge water. From these results, an independent measure of groundwater travel times to nearby wells could be determined. 


\subsubsection{Surface Water $\delta D$ and $\delta^{18} O$ Data}

The $\delta^{18} \mathrm{O}$ values for SAR water varied a modest $\sim 0.7$ per mil over 550 days from spring 1995 to late summer of 1996, ranging from -7.5 to -8.2 per mil (Fig. 10). SAR water measured at Imperial Headgates showed a general decrease in its $\delta^{18} \mathrm{O}$ over that time, with only two increases occurring in the spring and late summer of 1996. During baseflow periods, the majority of SAR flow originates from discharge of upstream tertiary-treated waste water. The $\delta^{18} \mathrm{O}$ values of these discharges are similar, around -8.5 per mil (Williams, 1994), due to common water sources (e.g. groundwater and SWP) used upstream. The lowest SAR value (-8.2 per mil) was recorded in the summer of 1996, when approximately 15,000 acre-ft of State Water Project (SWP) water was purchased and discharged in the SAR (see Table 3).

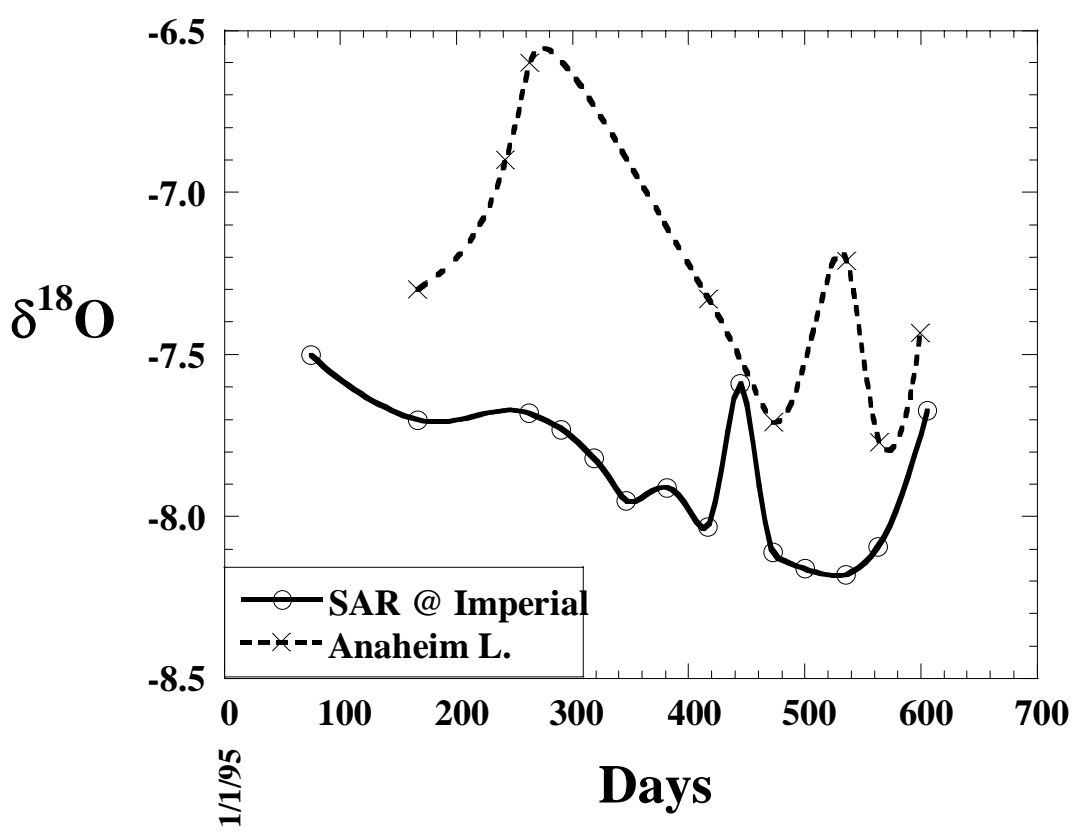

Figure 10. The $\delta^{18} \mathrm{O}$ of SAR showed a general decrease with time with two excursions to higher values during period of observation. Anaheim Lake has a somewhat higher value to due evaporative enrichment of the ${ }^{18} \mathrm{O}$.

During rainfall events, SAR flow originates as surface runoff from within the watershed. For large precipitation events, particularly late in the winter, runoff is stored behind Prado Dam. Samples of SAR discharge collected for three precipitation events by the US Geological Survey below Prado Dam and measured for $\delta^{18} \mathrm{O}$ by LLNL, revealed that the $\delta^{18} \mathrm{O}$ can significantly increase over a relatively short period of time (Fig. 11). This increase and subsequent variation during a single storm event are likely due to changes in runoff sources and were captured in the samples collected at different times below the dam. Even when there is storage behind Prado Dam these variations are still observed (Fig 11). 
Table 3. Annual Imported Water Volume Used for Recharge in Forebay.

\begin{tabular}{|c|c|c|}
\hline $\begin{array}{c}\text { Ending Water } \\
\text { Year }\end{array}$ & $\begin{array}{c}\text { Colorado River } \\
\text { Recharged (acre-ft) }\end{array}$ & $\begin{array}{l}\text { State Water Project } \\
\text { Recharge (acre-ft) }\end{array}$ \\
\hline 1950 & 22,726 & 0 \\
\hline 1951 & 22,183 & 0 \\
\hline 1952 & 39,177 & 0 \\
\hline 1953 & 27,956 & 0 \\
\hline 1954 & 50,000 & 0 \\
\hline 1955 & 67,789 & 0 \\
\hline 1956 & 20,916 & 0 \\
\hline 1957 & 82,955 & 0 \\
\hline 1958 & 77,145 & 0 \\
\hline 1959 & 81,710 & 0 \\
\hline 1960 & 136,042 & 0 \\
\hline 1961 & 166,279 & 0 \\
\hline 1962 & 173,149 & 0 \\
\hline 1963 & 216,960 & 0 \\
\hline 1964 & 185,440 & 0 \\
\hline 1965 & 132,270 & 0 \\
\hline 1966 & 116,460 & 0 \\
\hline 1967 & 114,050 & 0 \\
\hline 1968 & 91,797 & 0 \\
\hline 1969 & 52,354 & 0 \\
\hline 1970 & 84,938 & 0 \\
\hline 1971 & 55,575 & 0 \\
\hline 1972 & 34,327 & 0 \\
\hline 1973 & 52,871 & 4,142 \\
\hline 1974 & 48,292 & 42,796 \\
\hline 1975 & 51,801 & 47,159 \\
\hline 1976 & 14,908 & 72,211 \\
\hline 1977 & 15,138 & 16,306 \\
\hline 1978 & 58,660 & 1,916 \\
\hline 1979 & 19,412 & 10,266 \\
\hline 1980 & 34,643 & 8,138 \\
\hline 1981 & 33,108 & 0 \\
\hline 1982 & 37,575 & 0 \\
\hline 1983 & 14,821 & 0 \\
\hline 1984 & 15,114 & 0 \\
\hline 1985 & 32,010 & 0 \\
\hline 1986 & 30,235 & 0 \\
\hline 1987 & 27,626 & 0 \\
\hline 1988 & 39,634 & 1,181 \\
\hline 1989 & 2,897 & 7,865 \\
\hline 1990 & 27,395 & 3,306 \\
\hline 1991 & 15,619 & 0 \\
\hline 1992 & 51,672 & 0 \\
\hline 1993 & 26,293 & 0 \\
\hline 1994 & 78,521 & 0 \\
\hline 1995 & 14,668 & 3,094 \\
\hline 1996 & 0 & 15,279 \\
\hline
\end{tabular}



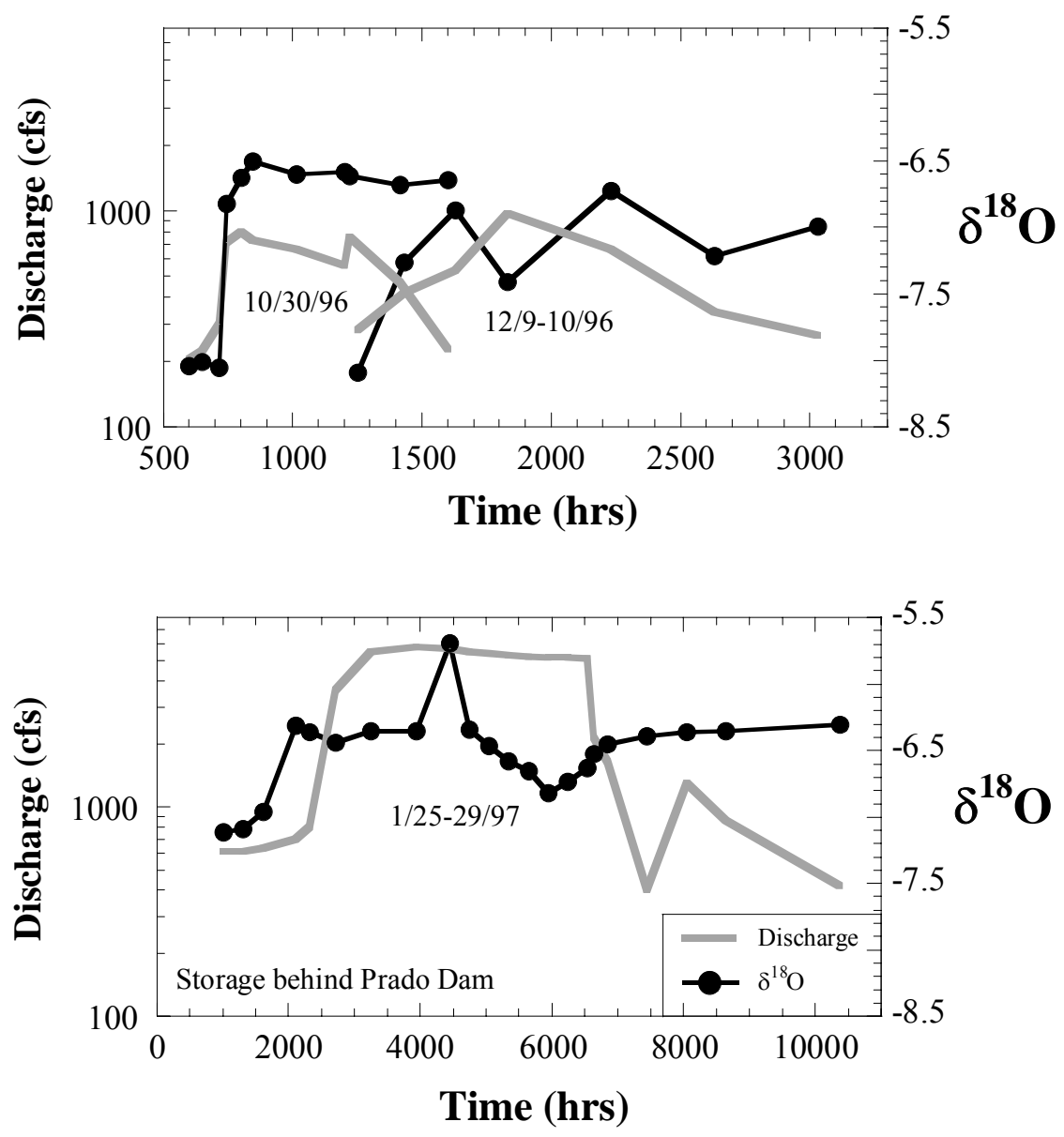

Figure 11. Storm flow runoff has large variation in $\delta^{18} \mathrm{O}$ due to changes in storm character and runoff source.

These variations may be related to limited mixing behind Prado as water from different runoff sources coalesce behind the dam. This limited mixing was observed during unusually high rainfall and runoff of the El Nino period in spring of 1998. During this time, approximately 25,000 acre-ft of water was in storage behind Prado Dam. Vertical profiles of $\mathrm{pH}$, conductivity, and temperature collected by District in several locations behind Prado Dam at this time revealed significant variation in the parameters with depth, consistent with limited vertical mixing (Fig. 12). 

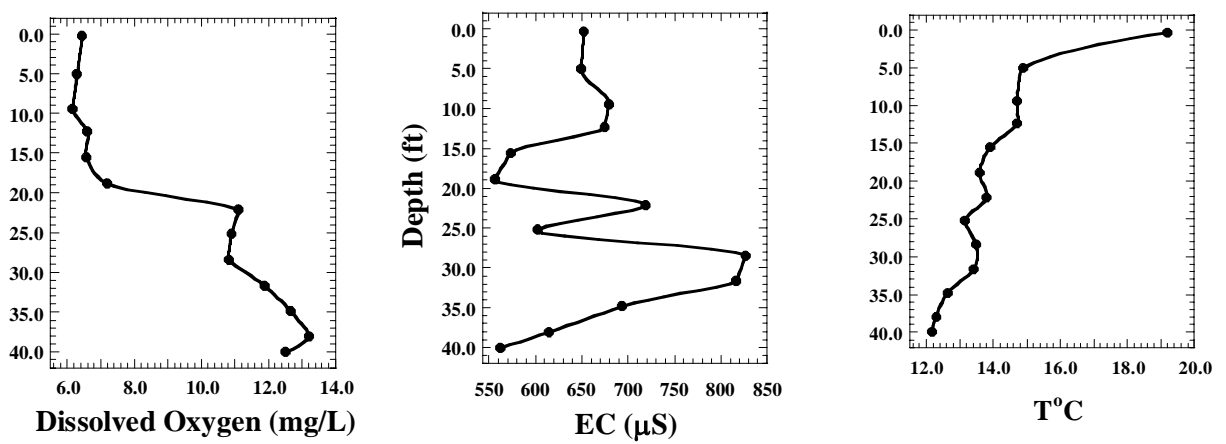

Figure 12. Even though approximately 25,000 acre-ft was stored behind Prado Dam, these field measurements suggest that water is stratified in the impounded reservoir due to limited vertical mixing. Data collected in March, 1998.

The $\delta^{18} \mathrm{O}$ of Anaheim Lake water had a somewhat larger variation than SAR water during spring 1995 to summer 1996 (Fig. 10). In particular, the $\delta^{18} \mathrm{O}$ values were higher in the summer/fall for Anaheim Lake water compared to the SAR. Except when Colorado River water (lower $\delta^{18} \mathrm{O}$ ) was diverted, Anaheim Lake water was derived exclusively from the SAR. The higher $\delta^{18} \mathrm{O}$ value during summer months likely resulted from evaporative enrichment of the lake water. This is also apparent in Figure 13, which shows the $\delta^{18} \mathrm{O}$ value at different depths for Anaheim Lake, Warner Basin, and Kraemer Basin collected in June 1996. All of these basins were recharging SAR water at the time. Even though each recharge basin has a similar depth, only Anaheim Lake has a distinctly higher $\delta^{18} \mathrm{O}$ in the upper 10 feet. In summer, lakes typically develop a thermocline due to surface water warming. If the residence time of water in the thermocline is long enough, significant evaporation and $\delta^{18} \mathrm{O}$ enrichment can occur. Anaheim Lake water likely has a high enough residence time to allow isotopic enrichment, whereas Kraemer Basin water is known to percolate faster than Anaheim Lake ( $\sim 3 \mathrm{ft} /$ day vs $\sim 1 \mathrm{ft} /$ day) and likely has insufficient residence time to allow $\delta^{18} \mathrm{O}$ evaporative enrichment. Warner Basin is not at a terminal point of SAR diversion, and is used as a throughflow basin. Thermocline development and any evaporative enrichment of $\delta^{18} \mathrm{O}$ in Warner Basin may depend on the season and SAR diversion operations.

Figure 13. Summer thermoclines are common in the upper 10-20 feet of lakes and are typically associated with increases in $\delta^{18} \mathrm{O}$ due to evaporation. Only Anaheim Lake shows a $\delta^{18} \mathrm{O}$ enrichment effect, while in Kraemer residence times may be shorter. Warner Basin is more complex due to through-flow, and more data is required to determine if $\delta^{18} \mathrm{O}$ enrichment occurs.

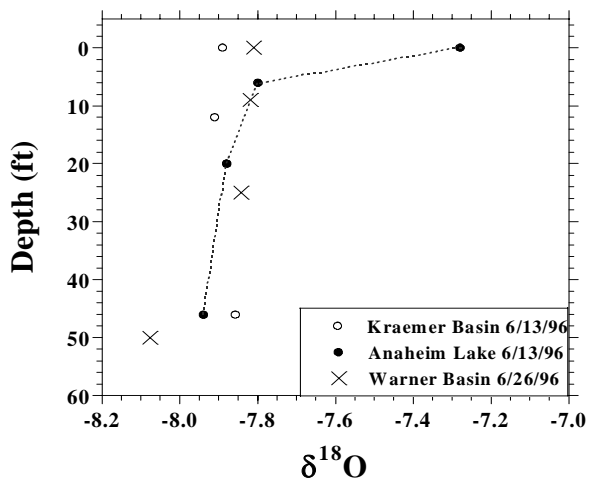




\subsubsection{Groundwater $\delta D$ and $\delta^{18} O$ Data}

Groundwater recharge has been grouped into those wells 1) adjacent to or just downgradient of the SAR, 2) those adjacent to or down gradient of Anaheim Lake/Kraemer basin east of highway 57,3) those downgradient of Santiago Pit, and 4) and those wells west of Highway 57. Earlier isotopic characterization and discussion of groundwater from these wells are also found in Davisson et al. (1996), Clemens-Knott et al., (1998), Davisson et al. (1999), and Yoshiba (1999).

7.2.2.1 Wells Adjacent to or Recharged Exclusively by the SAR - Wells measured in this vicinity are HG-1, YLWD-5, YLWD-11, YLWD-15, AM-6, SAR-6, and SAR-7. The $\delta^{18} \mathrm{O}$ values among these wells ranged from -7.3 to -8.5 per mil, similar to the range observed for the SAR, and consistent with recharge exclusively from this source.

Wells SAR-6 and SAR-7 were measured at multiple depths and both showed a measurable decrease $\left(\sim 0.5\right.$ per mil) in $\delta^{18} \mathrm{O}$ below approximately 850 feet. Repeat analysis of SAR-7 four months later revealed no change in $\delta^{18} \mathrm{O}$, indicating that the lower $\delta^{18} \mathrm{O}$ value observed below 850 feet was not related to a relatively rapid change at shallower depths. However, 850 feet below the SAR channel in this area is approximately the depth to bedrock, characterized by consolidated marine sediments (Herndon, 1992). These marine sediments are likely of much lower permeability, which would translate into slower groundwater transport rates. For example, in SAR-6 level 7, the ${ }^{3} \mathrm{H}$ was essentially non-detect, indicating an age $>40$ years old. However, in level 7 of SAR-7, the ${ }^{3} \mathrm{H}-{ }^{3} \mathrm{He}$ age was only approximately 9 years. The $\delta^{18} \mathrm{O}$ of SAR-7 level 7 is not as low as SAR-6 level 7, suggesting that SAR-7 level 7 is at the transition from higher to lower $\delta^{18} \mathrm{O}$ values. Unfortunately, levels deeper than SAR-7 level 7 were not age-dated, but likely are greater than nine years old.

Periodic samples for $\delta^{18} \mathrm{O}$ measurements were collected from wells HG-1 and YLWD11, starting in March of 1995 and continuing through the summer of 1996. The variation in $\delta^{18} \mathrm{O}$ in groundwater from these wells is apparently independent of changes in $\delta^{18} \mathrm{O}$ recorded in the SAR during the same sampling period. HG-1 and YLWD-11 had the same $\delta^{18} \mathrm{O}$ value $(-8.0$ per mil) at the beginning of sample collection period, but both were slightly lower than the SAR water at this same time (-7.7 per mil). The $\delta^{18} \mathrm{O}$ value of HG-1 increased over 0.5 per mil within the next 100 days, maintained this value through late 1995, and then oscillated between -7.3 and -8.3 per mil through the summer of 1996. YLWD-11 showed similar oscillation in $\delta^{18} \mathrm{O}$, but the magnitude of variation was smaller. Meanwhile, the SAR water only showed a general decrease in $\delta^{18} \mathrm{O}$ during this time with only two excursions to higher values in the spring and summer of 1996. These two excursions were not reflected in the data collected from HG-1 and YLWD-11.

The dissimilarity in $\delta^{18} \mathrm{O}$ of HG-1 and YLWD-11 from SAR water may simply be due to infrequent sampling of the SAR, and consequently, all records of isotopic variation in the river was not captured during this time period. Recharge water from the SAR would record surface water $\delta^{18} \mathrm{O}$ variations in an integrative manner. Recall that $\mathrm{HG}-1$ had a ${ }^{3} \mathrm{H}-$ ${ }^{3} \mathrm{He}$ age greater than one year, and likely reflects an integrated recharge $\delta^{18} \mathrm{O}$ value. Likewise, YLWD-11 was dated at 4 years old and would integrate more long-term recharge trends from the SAR. Recall also that repeat samples from HG-1 showed calculated recharge temperatures of 19 and $24^{\circ} \mathrm{C}$ (Table 1), indicating that distinct pulses 
of the SAR are recharged. In contrast, repeat analysis of YLWD-11 showed calculated recharge temperature of 13 and $15^{\circ} \mathrm{C}$, suggesting recharge records a temperature closer to mean annual air temperature (e.g. $14.1^{\circ} \mathrm{C}$ for Yorba Linda). In the case of HG-1, it is adjacent to the Imperial Headgates, which has an inflatable dam that is deployed at base flow to regulate stream discharge. Pooling of SAR water behind the dam will cause silting in the river bed over time and a decrease in recharge rates to HG-1. In the winter runoff periods, the dam is not inflated and river discharge scours the silt from the bed, causing increased percolation rates to $\mathrm{HG}-1$.

7.2.2.2 Wells Downgradient of Anaheim Lake and Kraemer Basin - Wells in this area measured for $\delta^{18} \mathrm{O}$ were AMD-9 levels 1 through 4, A-27, A-28, A-42, A-43, A-44, ABS2, AM-7, AM-9, AM-10, AM-14, OCWD-KB1, and SCWC-PLJ2. Wells A-27, A-28, and ABS-2 are located adjacent to Anaheim Lake, but have perforation levels completed across or above the low-permeability zone at $400 \mathrm{ft}$ bgs. Anaheim Lake recharge to these wells is anticipated, but exact quantities and travel times needed to be better understood. Monitoring well AMD-9 was completed in the summer of 1996 and has perforated intervals completed above (levels 1 and 2) and below (levels 3 and 4) the lowpermeability zone. The remaining wells are located downgradient of Anaheim Lake and Kraemer Basin, and have perforation levels in permeable strata that have lateral continuity with shallow aquifer layers located below both recharge basins.

Time series $\delta^{18} \mathrm{O}$ data were generated from spring 1995 to fall 1996 for wells A-27 and SCWC-PLJ2 (Figs. 14a-c). The initial repeat analysis of these two wells revealed that the $\delta^{18} \mathrm{O}$ value changed significantly over a relatively short period of time (SCWC-PLJ2 changed 0.4 per mil in 4 months, and A-27 changed 1.2 per mil in 4.5 months), therefore they were selected to be long-term monitoring points (see Davisson et al., 1996 for earlier discussion). Starting in March 1995, well A-27 increased in $\delta^{18} \mathrm{O}$ from -8.3 to -6.9 per mil over approximately 300 days. In general, this variation was similar to variations in $\delta^{18} \mathrm{O}$ of Anaheim Lake water during this same period. After 300 days, Anaheim Lake was emptied of water for approximately 100 days. While the basin was emptied, the $\delta^{18} \mathrm{O}$ in A-27 showed a significant decrease to -7.7 per mil. This value represented the isotopic value of a recharge source upgradient of Anaheim Lake, and is consistent with an SAR source. 

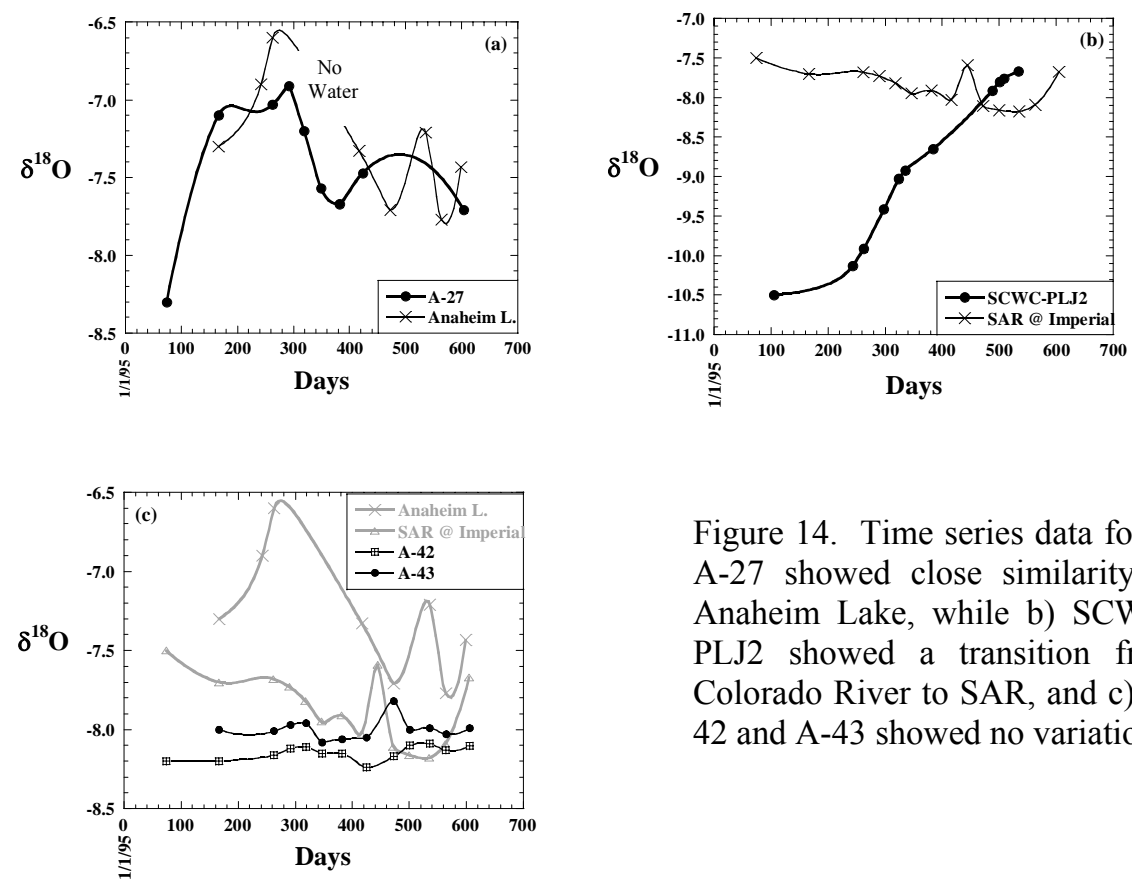

Figure 14. Time series data for a) A-27 showed close similarity to Anaheim Lake, while b) SCWCPLJ2 showed a transition from Colorado River to SAR, and c) A42 and $\mathrm{A}-43$ showed no variation.

Well SCWC-PLJ2 initially had a $\delta^{18} \mathrm{O}$ value of -10.5 per mil, but steadily increased to approximately -7.7 per mil in about 400 days. The initial $\delta^{18} \mathrm{O}$ indicated a significant mixture of Colorado River water, and the subsequent increase recorded a transition to an SAR water source. The total time required for this change was approximately 18 months.

Time-series analysis performed on samples from wells A-42 and A-43 showed that no measurable variation in $\delta^{18} \mathrm{O}$ occurred during almost 500 days of observation (Fig. 14c). The $\delta^{18} \mathrm{O}$ values for A-42 and A-43 were similar to values observed in the YLWD wells (approximately -8.0 per mil) and suggest an SAR recharge source. Repeat analysis on deep well A-44, however, showed a 0.4 per mil increase within an approximately 2 month period. This shift was significant enough to indicate a small change in the recharge source.

Depth-specific samples collected during spinner log tests for production wells A-42, A-43, and A-44 in February 1997 were also measured for $\delta^{18} \mathrm{O}$. The $\delta^{18} \mathrm{O}$ variation was less than 0.4 per mil in samples collected from four discrete depths in wells A-42 and A43 (Fig. 8a). However, in well A-44 the variation with depth was 1.0 per mil, ranging from -8.9 per mil at the bottom to -7.9 per mil at the top. Recall also that the ${ }^{3} \mathrm{H}-{ }^{3} \mathrm{He}$ age in depth specific samples of A-44 showed a systematic increase with depth, and the shallowest perforated interval of A-44 comprised the majority of flow in the well discharge (West, 1997).

Analyses of $\delta^{18} \mathrm{O}$ were also performed on the composite flows in each of these wells collected from the main pump discharge. In wells A-42 and A-43, the composite samples were approximately the same as the averages of $\delta^{18} \mathrm{O}$ values measured at the four different depths. However, the composite $\delta^{18} \mathrm{O}$ value for well A-44 was most similar to the shallowest level, as would be expected from the spinner log results. 
Repeat analyses were also performed on wells AM-7, AM-9, ABS-2, and OCWDKB1. Well AM-9 changed from -9.5 per mil in June, 1995 to -7.9 per mil in July, 1996. ABS-2 also changed from -9.4 per mil in March, 1995 to -7.9 per mil in October 1996. These $\delta^{18} \mathrm{O}$ changes in AM-9 and ABS-2 over approximately one year illustrated a transition from a Colorado River water mixture to water derived from the SAR. Well AM-10 was sampled in April 1995 and was -9.7 per mil, indicating a Colorado River water mixture. Surprisingly, well AM-13, measured in May 1997, was -9.2 per mil, indicating a Colorado River mixture in a south-southwest direction from Anaheim Lake and Kraemer Basin. Note that the ${ }^{3} \mathrm{H}-{ }^{3} \mathrm{He}$ age for $\mathrm{AM}-13$ is 3 years, suggesting the Colorado River water was related to 1994 recharge.

\subsection{Radiocarbon and Carbon-13 Results}

The objective of these measurements was to determine if pockets of relatively older groundwater existed in the Forebay, and if so what is their significance to overall recharge dynamics. The ${ }^{14} \mathrm{C}$ content of surface water measured in several locations exceeded 100 percent modern carbon (pmc), indicating modern water of post nuclear age (ca. 1950) The small variation from 103 to 107 pmc in all the surface water measured likely reflects variable contributions from inorganic solid carbon sources, for example carbonates, which occur as disseminated precipitates in soil or in concrete. The ${ }^{14} \mathrm{C}$ concentrations of surface water are lower than ${ }^{14} \mathrm{C}$ of modern atmospheric $\mathrm{CO}_{2}$ at the time of sampling $(\sim 112 \mathrm{pmc})$, suggesting small contribution from these type of carbon sources, which would have older ages.

The ${ }^{14} \mathrm{C}$ content measured in groundwater ranged from 62 to 115 pmc. Well waters with ${ }^{14} \mathrm{C}$ less than 100 pmc included F-AIRP, A-47, EOCW-E, O-23, OPWC, AM-10, and AM-6. AM-6 was 99 pmc, suggesting essentially a modern recharge source. AM-10 was $97 \mathrm{pmc}$ and consisted of a Colorado River water mixed with SAR, based on the $\delta^{18} \mathrm{O}$ results. Its ${ }^{14} \mathrm{C}$ was not unusually low, and it suggests small amounts of inorganic carbonate of low ${ }^{14} \mathrm{C}$ content may have contributed to the dissolved inorganic carbon of this groundwater.

F-AIRP and A-47 had ${ }^{14} \mathrm{C}$ content of 62 and $84 \mathrm{pmc}$, respectively. They are located in the western-most part of the Forebay and are perforated at depths greater than 400 feet. Their ${ }^{14} \mathrm{C}$ content is consistent with older groundwater, and their $\delta^{13} \mathrm{C}$ values $(-11.8$ and 10.2 per mil) do not suggest significant ${ }^{14} \mathrm{C}$-depleted carbonate dissolution contributed to the dissolved inorganic carbon. Note also that both F-AIRP and A-47 had measurable but low ${ }^{3} \mathrm{H}$, suggesting these wells produce both young (post-ca. 1963) and old groundwater (up to 4000 years old for F-AIRP). In the case of F-AIRP, the ${ }^{3} \mathrm{He} /{ }^{4} \mathrm{He}$ ratio indicated a contribution of radiogenic ${ }^{4} \mathrm{He}$ and is consistent with having the lowest ${ }^{14} \mathrm{C}$ content.

Wells EOCW-E, O-23, and OPWC had a ${ }^{14} \mathrm{C}$ content of 78,85 , and $87 \mathrm{pmc}$, respectively. Their $\delta^{13} \mathrm{C}$ values ranged from -10.1 to -11.5 per mil, and do not suggest extensive ${ }^{14} \mathrm{C}$-depleted carbonate contribution. The ${ }^{14} \mathrm{C}$ content of the groundwater is consistent with an age $\leq 2000$ years old. Note also that EOCW-E and O-23 both had measurable ${ }^{3} \mathrm{H}$ and ${ }^{3} \mathrm{He} /{ }^{4} \mathrm{He}$ ratios $>1$, indicating most of the groundwater from these wells is of young age (post-ca. 1963). 


\section{Research Results on the Artificial Tracer Experiments}

\subsection{Anaheim Lake Recharge Experiment}

The results from the environmental isotope characterization and age-dating study illustrated the rapid westward transport of Anaheim Lake, Kraemer Basin, and SAR recharge water. In particular, groundwater in most production wells within two miles of these recharge sources had ${ }^{3} \mathrm{H}^{-}{ }^{3} \mathrm{He}$ ages of less than five years. In some wells (e.g. A-28, A-44) mixtures of younger and older water are apparent, suggesting fast flow paths could reach production well perforation intervals. Groundwater produced from a well could incorporate a mixture of water with an age of less than one year, but have a measured ${ }^{3} \mathrm{H}$ ${ }^{3} \mathrm{He}$ age much older because mixing of older tritiated water. As a result, it was determined that the best approach to elucidate fast flow path components incorporated in groundwater wells was to conduct artificial recharge experiments using recharge water with a tracer that could be detected at low concentrations. In addition, this tracer would have to conform to strict water quality guidelines and not compromise potable uses. Isotopically-enriched xenon gas met these criteria. This is because the necessary amount of isotopically-enriched xenon needed to significantly perturb the natural xenon isotope abundance already dissolved in water only required an increase of the total dissolved xenon concentration by a factor of approximately two. Furthermore, xenon is nonreactive and is routinely used in medical procedures at doses far exceeding that potentially represented by the tracer. The disadvantage to a xenon tracer includes low solubility in water and the requirement of a noble gas mass spectrometer to analyze its isotopic abundance. Such instruments are not routinely available commercially. The solubility issue was overcome by spiking basin water at a depth great enough that daily percolation (feet per day) recharged most of the tracer and loss by diffusion to the surface was limited.

\subsubsection{Recharge Condition and Setup}

Starting October 1, 1996 approximately 6300 acre-ft of Colorado River water was purchased and discharged into Anaheim Lake at the OC-28 outlet over a 50 day period. Before Colorado River water was added, the lake was drained of remaining SAR water and scraped to remove fine particulate deposits. During the recharge experiment Kraemer Basin and Miller Basin received SAR water. Colorado River water was added to Anaheim Lake at approximately 100 cubic feet per second (cfs), which resulted in an average percolation rate of approximately $2.0 \mathrm{ft} /$ day. The lake depth was $43 \mathrm{ft}$ when the xenon tracer was added.

On October 11, 1996, the ${ }^{124} \mathrm{Xe}$ isotope tracer was added at several points in Anaheim Lake using a small boat. The tracer package consisted of a 2-liter stainless steel cylinder with valves on each end. Inside was approximately 500 milligrams of ${ }^{124} \mathrm{Xe}$-enriched gas that was pre-dissolved in approximately two liters of water. When these two liters were added to the stored water ( 1245 acre- $\mathrm{ft})$ the expected concentration of a well mixed tracer would be $4.7 \times 10^{-12} \mathrm{mmol}^{124} \mathrm{Xe} / \mathrm{mmolH}_{2} \mathrm{O}$. A greater than 25 foot length of Tygon ${ }^{\mathrm{TM}}$ tubing was attached to one end of the cylinder and lowered down into the basin using a weighted end. Using a peristaltic pump, the ${ }^{124} \mathrm{Xe}$-labelled water was pumped out of the cylinder through the Tygon ${ }^{\mathrm{TM}}$ tube to a depth greater than 15 feet in the lake. Given the rapid percolation rate of the basin at the time the tracer was added ( $>2.5 \mathrm{ft} / \mathrm{day})$, the loss of the Xe tracer from vertical diffusion to the surface was anticipated to be a minimal. 
The lateral mixing of the tracer was rapid, as indicated by four nearly identical concentrations of ${ }^{124} \mathrm{Xe}$ (approximately $2 \times 10^{-14}{ }^{124} \mathrm{Xe} \mathrm{mmol} / \mathrm{mmol} \mathrm{H}_{2} \mathrm{O}$ ) measured at various locations in the lake approximately 24 hours later (Table 4). Note that the measured concentration was less than half of what was expected in a well mixed condition. The $\delta^{18} \mathrm{O}$ value of these Anaheim Lake water samples was $-11.2 \pm 0.1$ per mil.

Groundwater wells up to two miles downgradient were monitored for both $\delta^{18} \mathrm{O}$ and ${ }^{124} \mathrm{Xe}$ (Table 4). Wells were monitored for approximately 300 days, with emphasis in the first 200 days on monitoring and production wells with 1000 feet of the lake. For shallow wells A-27, A-28, ABS-2, and AMD-9 level 1, whose perforation depths range from 155 to $361 \mathrm{ft}$ bgs, tracer arrival was anticipated to potentially be rapid, and consequently sampling was conducted on a daily to weekly basis. For outlying wells, sampling was less frequent.

\subsection{2 $\delta^{18} \mathrm{O}$ Results}

The Colorado River water arrived in wells A-27, A-28, ABS-2, and AMD-9 level 1 within 15-30 days after October 1 when Colorado River was first introduced into Anaheim Lake. For A-27 and AMD-9 level 1, the $\delta^{18}$ O changed rapidly from an SAR value to a Colorado River value in approximately 20 days (Fig. 15a). At that point, the $\delta^{18} \mathrm{O}$ of groundwater in AMD-9 level 1 reached approximately the same value (-11.3 per mil) as measured in Anaheim Lake. At the same time, the $\delta^{18} \mathrm{O}$ value of groundwater in A-27 was slightly higher (-11.0 per mil) than Anaheim Lake. Both wells returned to an SAR isotopic value within 60 days of when flow of Colorado River water into Anaheim Lake was terminated.
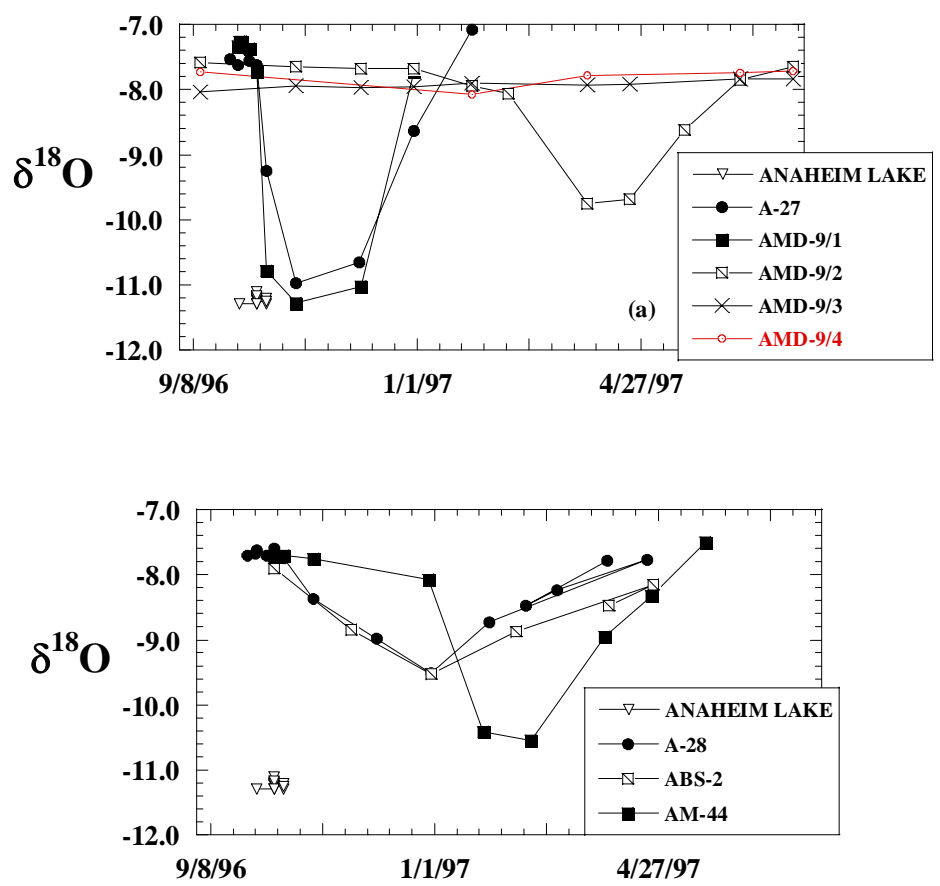

Figure 15. Breakthrough curves of Colorado River from Anaheim Lake show a) 30 day turnover of $100 \%$ of the groundwater in wells A-27 and AMD-9 level 1, and b) higher dispersion for A-28 and ABS-2. 
Table 4. Xenon Tracer Results from 1996 and 1998 Recharge Experiments.

\begin{tabular}{|c|c|c|c|c|c|c|c|c|}
\hline \multirow{2}{*}{ Sample } & \multirow{2}{*}{$\begin{array}{l}\text { Sample } \\
\text { Date }\end{array}$} & \multicolumn{2}{|c|}{${ }^{124} \mathrm{Xe} \quad{ }^{128} \mathrm{Xe}$} & ${ }^{129} \mathrm{Xe}$ & ${ }^{136} \mathrm{Xe}$ & $\mathrm{C} / \mathrm{Co}{ }^{136} \mathrm{Xe}$ & \multirow{2}{*}{$\begin{array}{l}\mathrm{C} / \mathrm{Co}{ }^{129} \mathrm{Xe} \\
\text { as percent }\end{array}$} & \multirow{2}{*}{$\begin{array}{l}\mathrm{C} / \mathrm{Co}^{124} \mathrm{Xe} \\
\text { as percent }\end{array}$} \\
\hline & & $\mathrm{mol} / \mathrm{mol} \mathrm{H}_{2} \mathrm{O}$ & 2 & $\mathbf{H}_{2}$ & $\mathrm{ol} / \mathrm{mol} \mathrm{H}_{2} \mathrm{O}$ & as percent & & \\
\hline A26 & 4/1/1997 & $3.56 \mathrm{E}-18$ & $0.00 \mathrm{E}+00$ & $0.00 \mathrm{E}+00$ & $0.00 \mathrm{E}+00$ & 0.0000 & 0.0000 & 0.0175 \\
\hline A26 & $7 / 22 / 1998$ & $8.15 \mathrm{E}-18$ & $1.39 \mathrm{E}-16$ & $4.91 \mathrm{E}-16$ & $1.63 \mathrm{E}-16$ & 0.0241 & 0.2488 & 0.0400 \\
\hline A26 & $9 / 1 / 1998$ & $3.98 \mathrm{E}-17$ & $1.86 \mathrm{E}-16$ & $0.00 \mathrm{E}+00$ & $0.00 \mathrm{E}+00$ & 0.0000 & 0.0000 & 0.1953 \\
\hline A26 & $10 / 28 / 1998$ & $0.00 \mathrm{E}+00$ & $0.00 \mathrm{E}+00$ & $0.00 \mathrm{E}+00$ & $0.00 \mathrm{E}+00$ & 0.0000 & 0.0000 & 0.0000 \\
\hline A26 & $12 / 22 / 1998$ & $0.00 \mathrm{E}+00$ & $0.00 \mathrm{E}+00$ & $0.00 \mathrm{E}+00$ & $0.00 \mathrm{E}+00$ & 0.0000 & 0.0000 & 0.0000 \\
\hline A26 & $2 / 18 / 1999$ & $1.61 \mathrm{E}-17$ & $1.22 \mathrm{E}-16$ & $4.74 \mathrm{E}-16$ & $1.02 \mathrm{E}-16$ & 0.0151 & 0.2403 & 0.0788 \\
\hline A26 & $5 / 12 / 1999$ & $0.00 \mathrm{E}+00$ & $0.00 \mathrm{E}+00$ & $0.00 \mathrm{E}+00$ & 4.19E-16 & 0.0619 & 0.0000 & 0.0000 \\
\hline A26 & 6/9/1999 & $0.00 \mathrm{E}+00$ & $2.52 \mathrm{E}-16$ & $0.00 \mathrm{E}+00$ & $1.26 \mathrm{E}-15$ & 0.1863 & 0.0000 & 0.0000 \\
\hline A26 & 7/8/1999 & $3.22 \mathrm{E}-17$ & $0.00 \mathrm{E}+00$ & 7.07E-16 & $2.26 \mathrm{E}-15$ & 0.3350 & 0.3585 & 0.1581 \\
\hline A26 & 8/3/1999 & $0.00 \mathrm{E}+00$ & $0.00 \mathrm{E}+00$ & $8.40 \mathrm{E}-16$ & $3.77 \mathrm{E}-15$ & 0.5579 & 0.4258 & 0.0000 \\
\hline A26 & $9 / 10 / 1999$ & $0.00 \mathrm{E}+00$ & $0.00 \mathrm{E}+00$ & $0.00 \mathrm{E}+00$ & $1.31 \mathrm{E}-15$ & 0.1936 & 0.0000 & 0.0000 \\
\hline A26 & $9 / 27 / 1999$ & $0.00 \mathrm{E}+00$ & $0.00 \mathrm{E}+00$ & $0.00 \mathrm{E}+00$ & $7.45 \mathrm{E}-15$ & 1.1020 & 0.0000 & 0.0000 \\
\hline A26 & 10/28/1999 & $5.34 \mathrm{E}-17$ & $8.77 \mathrm{E}-16$ & $2.36 \mathrm{E}-14$ & $0.00 \mathrm{E}+00$ & 0.0000 & 11.9450 & 0.2622 \\
\hline A26 & $12 / 1 / 1999$ & $0.00 \mathrm{E}+00$ & $2.08 \mathrm{E}-16$ & $0.00 \mathrm{E}+00$ & $8.67 \mathrm{E}-15$ & 1.2834 & 0.0000 & 0.0000 \\
\hline A26 & $3 / 21 / 2000$ & $5.40 \mathrm{E}-17$ & $5.22 \mathrm{E}-16$ & $6.92 \mathrm{E}-15$ & $8.84 \mathrm{E}-15$ & 1.3076 & 3.5069 & 0.2653 \\
\hline A26 & $5 / 16 / 2000$ & $6.35 \mathrm{E}-17$ & $4.70 \mathrm{E}-16$ & $9.91 \mathrm{E}-16$ & $8.09 \mathrm{E}-15$ & 1.1967 & 0.5026 & 0.3117 \\
\hline A26 & $7 / 10 / 2000$ & $0.00 \mathrm{E}+00$ & $2.68 \mathrm{E}-18$ & $0.00 \mathrm{E}+00$ & $0.00 \mathrm{E}+00$ & 0.0000 & 0.0000 & 0.0000 \\
\hline A26 & $10 / 16 / 2000$ & $0.00 \mathrm{E}+00$ & $0.00 \mathrm{E}+00$ & $0.00 \mathrm{E}+00$ & $2.02 \mathrm{E}-15$ & 0.2987 & 0.0000 & 0.0000 \\
\hline A27 & $10 / 13 / 1998$ & $4.05 \mathrm{E}-17$ & $2.24 \mathrm{E}-16$ & $1.09 \mathrm{E}-15$ & $1.58 \mathrm{E}-16$ & 0.0234 & 0.5548 & 0.1989 \\
\hline A27 & $10 / 15 / 1998$ & $9.07 \mathrm{E}-17$ & $2.27 \mathrm{E}-15$ & $1.28 \mathrm{E}-14$ & $3.26 \mathrm{E}-15$ & 0.4820 & 6.4843 & 0.4455 \\
\hline A 27 & $10 / 21 / 1998$ & $4.46 \mathrm{E}-16$ & $1.94 \mathrm{E}-14$ & $1.01 \mathrm{E}-13$ & $4.30 \mathrm{E}-15$ & 0.6358 & 51.0100 & 2.1903 \\
\hline A27 & 10/30/1998 & $1.30 \mathrm{E}-16$ & $7.10 \mathrm{E}-15$ & $3.68 \mathrm{E}-14$ & $3.35 \mathrm{E}-15$ & 0.4956 & 18.6430 & 0.6373 \\
\hline A27 & $11 / 4 / 1998$ & $1.60 \mathrm{E}-16$ & $5.23 \mathrm{E}-15$ & $2.75 \mathrm{E}-14$ & $6.37 \mathrm{E}-15$ & 0.9427 & 13.9300 & 0.7864 \\
\hline A 27 & $11 / 10 / 1998$ & $9.04 \mathrm{E}-17$ & $4.65 \mathrm{E}-15$ & $2.43 \mathrm{E}-14$ & $1.40 \mathrm{E}-15$ & 0.2075 & 12.3190 & 0.4440 \\
\hline A27 & $11 / 20 / 1998$ & $2.62 \mathrm{E}-17$ & $2.14 \mathrm{E}-15$ & $1.07 \mathrm{E}-14$ & $5.73 \mathrm{E}-15$ & 0.8483 & 5.4314 & 0.1288 \\
\hline A27 & $12 / 2 / 1998$ & $9.69 \mathrm{E}-17$ & $1.14 \mathrm{E}-15$ & $7.28 \mathrm{E}-15$ & $3.41 \mathrm{E}-15$ & 0.5043 & 3.6929 & 0.4761 \\
\hline A27 & $12 / 7 / 1998$ & $2.79 \mathrm{E}-17$ & $1.17 \mathrm{E}-15$ & $5.28 \mathrm{E}-15$ & $4.77 \mathrm{E}-15$ & 0.7058 & 2.6767 & 0.1369 \\
\hline A28 & $10 / 11 / 1996$ & E-17 & $2.47 \mathrm{E}-16$ & $4.80 \mathrm{E}-16$ & E-16 & 0.0299 & 0.2433 & 0.1571 \\
\hline A28 & $10 / 31 / 1996$ & $9.22 \mathrm{E}-16$ & $3.09 \mathrm{E}-16$ & $7.32 \mathrm{E}-16$ & $1.67 \mathrm{E}-17$ & 0.0025 & 0.3713 & 4.5300 \\
\hline A28 & $12 / 3 / 1996$ & $2.20 \mathrm{E}-15$ & $3.68 \mathrm{E}-16$ & $1.60 \mathrm{E}-15$ & $9.31 \mathrm{E}-17$ & 0.0138 & 0.8137 & 10.7800 \\
\hline A28 & $12 / 31 / 1996$ & $1.76 \mathrm{E}-15$ & $3.80 \mathrm{E}-16$ & $1.27 \mathrm{E}-15$ & $7.07 \mathrm{E}-17$ & 0.0105 & 0.6462 & 8.6522 \\
\hline A28 & $1 / 30 / 1997$ & $1.24 \mathrm{E}-15$ & $5.61 \mathrm{E}-16$ & $1.34 \mathrm{E}-15$ & $3.05 \mathrm{E}-17$ & 0.0045 & 0.6779 & 6.0861 \\
\hline A28 & $3 / 5 / 1997$ & $6.33 \mathrm{E}-16$ & $3.14 \mathrm{E}-16$ & $2.57 \mathrm{E}-16$ & $0.00 \mathrm{E}+00$ & 0.0000 & 0.1304 & 3.1105 \\
\hline A28 & 4/1/1997 & $3.81 \mathrm{E}-16$ & $4.00 \mathrm{E}-16$ & $4.72 \mathrm{E}-16$ & $0.00 \mathrm{E}+00$ & 0.0000 & 0.2391 & 1.8688 \\
\hline A42 & $10 / 7 / 1996$ & $1.08 \mathrm{E}-17$ & $0.00 \mathrm{E}+00$ & $0.00 \mathrm{E}+00$ & $3.63 \mathrm{E}-17$ & 0.0054 & 0.0000 & 0.0529 \\
\hline A42 & $1 / 30 / 1997$ & $4.95 \mathrm{E}-17$ & $3.88 \mathrm{E}-16$ & $0.00 \mathrm{E}+00$ & $7.10 \mathrm{E}-17$ & 0.0105 & 0.0000 & 0.2433 \\
\hline A42 & $2 / 18 / 1997$ & $9.59 \mathrm{E}-17$ & $3.82 \mathrm{E}-16$ & $1.13 \mathrm{E}-15$ & $0.00 \mathrm{E}+00$ & 0.0000 & 0.5738 & 0.4711 \\
\hline A42 & 4/1/1997 & $0.00 \mathrm{E}+00$ & $3.64 \mathrm{E}-16$ & $0.00 \mathrm{E}+00$ & $0.00 \mathrm{E}+00$ & 0.0000 & 0.0000 & 0.0000 \\
\hline A42 & $4 / 22 / 1997$ & $7.48 \mathrm{E}-17$ & $2.42 \mathrm{E}-16$ & $0.00 \mathrm{E}+00$ & $1.12 \mathrm{E}-16$ & 0.0166 & 0.0000 & 0.3675 \\
\hline A42 & $5 / 20 / 1997$ & $0.00 \mathrm{E}+00$ & $2.85 \mathrm{E}-16$ & $0.00 \mathrm{E}+00$ & $8.11 \mathrm{E}-17$ & 0.0120 & 0.0000 & 0.0000 \\
\hline A42 & $7 / 15 / 1997$ & $4.26 \mathrm{E}-17$ & $8.87 \mathrm{E}-16$ & $1.28 \mathrm{E}-16$ & $0.00 \mathrm{E}+00$ & 0.0000 & 0.0650 & 0.2094 \\
\hline A42 & $7 / 15 / 1997$ & $0.00 \mathrm{E}+00$ & $3.18 \mathrm{E}-16$ & $0.00 \mathrm{E}+00$ & $0.00 \mathrm{E}+00$ & 0.0000 & 0.0000 & 0.0000 \\
\hline A42 & $9 / 24 / 1997$ & $0.00 \mathrm{E}+00$ & $2.63 \mathrm{E}-16$ & $0.00 \mathrm{E}+00$ & $0.00 \mathrm{E}+00$ & 0.0000 & 0.0000 & 0.0000 \\
\hline A42 & 10/21/1997 & $2.40 \mathrm{E}-17$ & $2.52 \mathrm{E}-16$ & $0.00 \mathrm{E}+00$ & $1.34 \mathrm{E}-16$ & 0.0198 & 0.0000 & 0.1180 \\
\hline A42 & $10 / 28 / 1998$ & $9.57 \mathrm{E}-18$ & $0.00 \mathrm{E}+00$ & $1.05 \mathrm{E}-15$ & $5.66 \mathrm{E}-15$ & 0.8372 & 0.5320 & 0.0470 \\
\hline A42 & $11 / 21 / 1998$ & $4.24 \mathrm{E}-17$ & $0.00 \mathrm{E}+00$ & $4.69 \mathrm{E}-16$ & $6.41 \mathrm{E}-15$ & 0.9480 & 0.2380 & 0.2080 \\
\hline A42 & $12 / 22 / 1998$ & $0.00 \mathrm{E}+00$ & $0.00 \mathrm{E}+00$ & $1.72 \mathrm{E}-16$ & $6.09 \mathrm{E}-15$ & 0.9017 & 0.0872 & 0.0000 \\
\hline A42 & $1 / 19 / 1999$ & $2.27 \mathrm{E}-17$ & 7.37E-17 & $6.92 \mathrm{E}-16$ & $0.00 \mathrm{E}+00$ & 0.0000 & 0.3508 & 0.1116 \\
\hline A42 & 2/18/1999 & $1.06 \mathrm{E}-16$ & $3.40 \mathrm{E}-17$ & $4.66 \mathrm{E}-16$ & $0.00 \mathrm{E}+00$ & 0.0000 & 0.2363 & 0.5191 \\
\hline
\end{tabular}


Table 4. Xenon Tracer Results from 1996 and 1998 Recharge Experiments.

\begin{tabular}{|c|c|c|c|c|c|c|c|c|}
\hline \multirow{2}{*}{ Sample } & \multirow{2}{*}{$\begin{array}{l}\text { Sample } \\
\text { Date }\end{array}$} & \multicolumn{2}{|c|}{${ }^{124} \mathrm{Xe} \quad{ }^{128} \mathrm{Xe}$} & ${ }^{129} \mathrm{Xe}$ & \multirow{2}{*}{${ }^{136} \mathrm{Xe}$} & $\mathrm{C} / \mathrm{Co}{ }^{136} \mathrm{Xe}$ & \multirow{2}{*}{$\begin{array}{l}\mathrm{C} / \mathrm{Co}{ }^{129} \mathrm{Xe} \\
\text { as percent }\end{array}$} & \multirow{2}{*}{$\begin{array}{l}\mathrm{C} / \mathrm{Co}^{124} \mathrm{Xe} \\
\text { as percent }\end{array}$} \\
\hline & & $\mathrm{mol} / \mathrm{mol} \mathrm{H}_{2} \mathrm{O}$ & $\mathrm{l} / \mathrm{mol} \mathrm{H}_{2}$ & $1 / \mathbf{m o l ~ H}_{2}$ & & as percent & & \\
\hline A42 & $3 / 16 / 1999$ & $3.79 \mathrm{E}-17$ & $0.00 \mathrm{E}+00$ & $1.56 \mathrm{E}-15$ & $2.41 \mathrm{E}-15$ & 0.3573 & 0.7892 & 0.1862 \\
\hline A42 & 4/15/1999 & $2.09 \mathrm{E}-18$ & $5.40 \mathrm{E}-17$ & $1.05 \mathrm{E}-15$ & $6.05 \mathrm{E}-15$ & 0.8947 & 0.5313 & 0.0103 \\
\hline A42 & $5 / 12 / 1999$ & $2.19 \mathrm{E}-17$ & $2.61 \mathrm{E}-16$ & $3.50 \mathrm{E}-15$ & $0.00 \mathrm{E}+00$ & 0.0000 & 1.7724 & 0.1076 \\
\hline A42 & $6 / 10 / 1999$ & $4.46 \mathrm{E}-17$ & $1.62 \mathrm{E}-16$ & $1.23 \mathrm{E}-15$ & $0.00 \mathrm{E}+00$ & 0.0000 & 0.6234 & 0.2191 \\
\hline A42 & 7/8/1999 & $3.85 \mathrm{E}-17$ & $4.83 \mathrm{E}-16$ & $4.00 \mathrm{E}-15$ & $7.14 \mathrm{E}-15$ & 1.0570 & 2.0263 & 0.1892 \\
\hline A42 & 8/3/1999 & $9.72 \mathrm{E}-17$ & $1.77 \mathrm{E}-16$ & $8.16 \mathrm{E}-16$ & $2.55 \mathrm{E}-15$ & 0.3775 & 0.4138 & 0.4772 \\
\hline A42 & 9/10/1999 & $4.69 \mathrm{E}-17$ & $5.53 \mathrm{E}-17$ & $1.90 \mathrm{E}-16$ & $6.79 \mathrm{E}-15$ & 1.0053 & 0.0962 & 0.2302 \\
\hline A42 & $9 / 27 / 1999$ & $1.24 \mathrm{E}-16$ & $2.57 \mathrm{E}-16$ & $5.85 \mathrm{E}-16$ & $7.19 \mathrm{E}-16$ & 0.1063 & 0.2968 & 0.6091 \\
\hline A43 & $10 / 7 / 1996$ & $0.00 \mathrm{E}+00$ & $0.00 \mathrm{E}+00$ & $0.00 \mathrm{E}+00$ & $4.40 \mathrm{E}-17$ & 0.0065 & 0.0000 & 0.0000 \\
\hline A43 & $1 / 30 / 1997$ & $0.00 \mathrm{E}+00$ & $2.13 \mathrm{E}-17$ & $0.00 \mathrm{E}+00$ & $0.00 \mathrm{E}+00$ & 0.0000 & 0.0000 & 0.0000 \\
\hline A43 & $1 / 30 / 1997$ & $0.00 \mathrm{E}+00$ & $0.00 \mathrm{E}+00$ & $0.00 \mathrm{E}+00$ & $0.00 \mathrm{E}+00$ & 0.0000 & 0.0000 & 0.0000 \\
\hline A43 & $2 / 18 / 1997$ & $3.70 \mathrm{E}-19$ & $7.14 \mathrm{E}-17$ & $0.00 \mathrm{E}+00$ & $0.00 \mathrm{E}+00$ & 0.0000 & 0.0000 & 0.0018 \\
\hline A43 & 4/2/1997 & $4.53 \mathrm{E}-17$ & $1.49 \mathrm{E}-16$ & $0.00 \mathrm{E}+00$ & $1.38 \mathrm{E}-16$ & 0.0204 & 0.0000 & 0.2225 \\
\hline A43 & $4 / 22 / 1997$ & $0.00 \mathrm{E}+00$ & $0.00 \mathrm{E}+00$ & $0.00 \mathrm{E}+00$ & $3.93 \mathrm{E}-16$ & 0.0582 & 0.0000 & 0.0000 \\
\hline A43 & $6 / 18 / 1997$ & $0.00 \mathrm{E}+00$ & $0.00 \mathrm{E}+00$ & $0.00 \mathrm{E}+00$ & $3.70 \mathrm{E}-16$ & 0.0548 & 0.0000 & 0.0000 \\
\hline A43 & $6 / 18 / 1997$ & $9.10 \mathrm{E}-17$ & $0.00 \mathrm{E}+00$ & $0.00 \mathrm{E}+00$ & $2.44 \mathrm{E}-16$ & 0.0361 & 0.0000 & 0.4470 \\
\hline A43 & $8 / 26 / 1997$ & $0.00 \mathrm{E}+00$ & $0.00 \mathrm{E}+00$ & $0.00 \mathrm{E}+00$ & $4.42 \mathrm{E}-16$ & 0.0654 & 0.0000 & 0.0000 \\
\hline A43 & 9/24/1997 & $0.00 \mathrm{E}+00$ & $0.00 \mathrm{E}+00$ & $0.00 \mathrm{E}+00$ & $3.26 \mathrm{E}-16$ & 0.0482 & 0.0000 & 0.0000 \\
\hline A43 & $10 / 24 / 1997$ & $1.88 \mathrm{E}-19$ & $0.00 \mathrm{E}+00$ & $0.00 \mathrm{E}+00$ & $1.59 \mathrm{E}-16$ & 0.0235 & 0.0000 & 0.0009 \\
\hline A43 & 11/13/1997 & $6.22 \mathrm{E}-17$ & $1.14 \mathrm{E}-16$ & $0.00 \mathrm{E}+00$ & $2.56 \mathrm{E}-16$ & 0.0379 & 0.0000 & 0.3055 \\
\hline A43 & $12 / 22 / 1998$ & $0.00 \mathrm{E}+00$ & $1.34 \mathrm{E}-16$ & $0.00 \mathrm{E}+00$ & $2.34 \mathrm{E}-15$ & 0.3467 & 0.0000 & 0.0000 \\
\hline A43 & $2 / 24 / 1999$ & $4.10 \mathrm{E}-17$ & $3.44 \mathrm{E}-17$ & $7.41 \mathrm{E}-17$ & $0.00 \mathrm{E}+00$ & 0.0000 & 0.0376 & 0.2012 \\
\hline A43 & 4/15/1999 & $0.00 \mathrm{E}+00$ & $0.00 \mathrm{E}+00$ & $0.00 \mathrm{E}+00$ & $3.85 \mathrm{E}-15$ & 0.5702 & 0.0000 & 0.0000 \\
\hline A43 & $6 / 10 / 1999$ & $9.13 \mathrm{E}-18$ & $0.00 \mathrm{E}+00$ & $2.17 \mathrm{E}-16$ & $8.73 \mathrm{E}-15$ & 1.2918 & 0.1101 & 0.0449 \\
\hline A43 & 8/3/1999 & $1.27 \mathrm{E}-17$ & $0.00 \mathrm{E}+00$ & $6.54 \mathrm{E}-16$ & $3.84 \mathrm{E}-15$ & 0.5676 & 0.3315 & 0.0624 \\
\hline A43 & 8/7/1999 & $3.75 \mathrm{E}-17$ & $1.41 \mathrm{E}-17$ & $0.00 \mathrm{E}+00$ & $9.11 \mathrm{E}-15$ & 1.3480 & 0.0000 & 0.1843 \\
\hline A43 & $9 / 10 / 1999$ & $0.00 \mathrm{E}+00$ & $1.57 \mathrm{E}-16$ & $1.12 \mathrm{E}-15$ & $1.35 \mathrm{E}-15$ & 0.1997 & 0.5701 & 0.0000 \\
\hline A43 & $9 / 27 / 1999$ & $7.27 \mathrm{E}-17$ & $2.93 \mathrm{E}-17$ & $8.66 \mathrm{E}-16$ & $5.92 \mathrm{E}-15$ & 0.8755 & 0.4392 & 0.3569 \\
\hline A43 & 10/28/1999 & $8.64 \mathrm{E}-17$ & $0.00 \mathrm{E}+00$ & $0.00 \mathrm{E}+00$ & $2.27 \mathrm{E}-15$ & 0.3364 & 0.0000 & 0.4243 \\
\hline A44 & $5 / 23 / 1996$ & $0.00 \mathrm{E}+00$ & $2.65 \mathrm{E}-16$ & $0.00 \mathrm{E}+00$ & $2.40 \mathrm{E}-16$ & 0.0355 & 0.0000 & 0.0000 \\
\hline A44 & $10 / 7 / 1996$ & $7.81 \mathrm{E}-17$ & $2.12 \mathrm{E}-16$ & $0.00 \mathrm{E}+00$ & $0.00 \mathrm{E}+00$ & 0.0000 & 0.0000 & 0.3835 \\
\hline A44 & $12 / 31 / 1996$ & $0.00 \mathrm{E}+00$ & $4.37 \mathrm{E}-17$ & $0.00 \mathrm{E}+00$ & $3.22 \mathrm{E}-17$ & 0.0048 & 0.0000 & 0.0000 \\
\hline A44 & $2 / 14 / 1997$ & $0.00 \mathrm{E}+00$ & $3.06 \mathrm{E}-16$ & $0.00 \mathrm{E}+00$ & $3.12 \mathrm{E}-16$ & 0.0462 & 0.0000 & 0.0000 \\
\hline A44 & $3 / 5 / 1997$ & $0.00 \mathrm{E}+00$ & $3.89 \mathrm{E}-16$ & $0.00 \mathrm{E}+00$ & $1.47 \mathrm{E}-16$ & 0.0218 & 0.0000 & 0.0000 \\
\hline A44 & 4/1/1997 & 4.10E-16 & $1.12 \mathrm{E}-15$ & $0.00 \mathrm{E}+00$ & $0.00 \mathrm{E}+00$ & 0.0000 & 0.0000 & 2.0141 \\
\hline A44 & $4 / 22 / 1997$ & $5.95 \mathrm{E}-16$ & $1.95 \mathrm{E}-16$ & $0.00 \mathrm{E}+00$ & $2.15 \mathrm{E}-16$ & 0.0318 & 0.0000 & 2.9228 \\
\hline A44 & $4 / 22 / 1997$ & $6.46 \mathrm{E}-16$ & $0.00 \mathrm{E}+00$ & $0.00 \mathrm{E}+00$ & $5.76 \mathrm{E}-16$ & 0.0852 & 0.0000 & 3.1727 \\
\hline A44 & $5 / 20 / 1997$ & $4.43 \mathrm{E}-16$ & $1.36 \mathrm{E}-16$ & $0.00 \mathrm{E}+00$ & $5.20 \mathrm{E}-16$ & 0.0769 & 0.0000 & 2.1769 \\
\hline A44 & $5 / 20 / 1997$ & $4.65 \mathrm{E}-16$ & $0.00 \mathrm{E}+00$ & $0.00 \mathrm{E}+00$ & $4.68 \mathrm{E}-16$ & 0.0693 & 0.0000 & 2.2854 \\
\hline A44 & $6 / 18 / 1997$ & $4.02 \mathrm{E}-16$ & $2.33 \mathrm{E}-16$ & $0.00 \mathrm{E}+00$ & $3.06 \mathrm{E}-16$ & 0.0452 & 0.0000 & 1.9762 \\
\hline A44 & $7 / 15 / 1997$ & $2.34 \mathrm{E}-16$ & $3.33 \mathrm{E}-17$ & $0.00 \mathrm{E}+00$ & $3.09 \mathrm{E}-16$ & 0.0457 & 0.0000 & 1.1481 \\
\hline A44 & $8 / 26 / 1997$ & $9.32 \mathrm{E}-17$ & $2.23 \mathrm{E}-16$ & $0.00 \mathrm{E}+00$ & $1.56 \mathrm{E}-16$ & 0.0230 & 0.0000 & 0.4579 \\
\hline A44 & 9/24/1997 & $5.02 \mathrm{E}-17$ & $7.24 \mathrm{E}-16$ & $0.00 \mathrm{E}+00$ & $1.78 \mathrm{E}-16$ & 0.0263 & 0.0000 & 0.2468 \\
\hline A44 & $10 / 21 / 1997$ & $8.47 \mathrm{E}-17$ & $3.21 \mathrm{E}-16$ & $0.00 \mathrm{E}+00$ & $1.31 \mathrm{E}-16$ & 0.0194 & 0.0000 & 0.4161 \\
\hline A44 & 11/13/1997 & $0.00 \mathrm{E}+00$ & $2.51 \mathrm{E}-16$ & $0.00 \mathrm{E}+00$ & $0.00 \mathrm{E}+00$ & 0.0000 & 0.0000 & 0.0000 \\
\hline A44 & $10 / 28 / 1998$ & $1.14 \mathrm{E}-16$ & $0.00 \mathrm{E}+00$ & $1.53 \mathrm{E}-16$ & $8.33 \mathrm{E}-15$ & 1.2325 & 0.0774 & 0.5575 \\
\hline A44 & $11 / 24 / 1998$ & $3.40 \mathrm{E}-17$ & $2.79 \mathrm{E}-16$ & $3.73 \mathrm{E}-15$ & $7.25 \mathrm{E}-16$ & 0.1073 & 1.8936 & 0.1672 \\
\hline A44 & $12 / 28 / 1998$ & $3.25 \mathrm{E}-17$ & $1.05 \mathrm{E}-16$ & $4.48 \mathrm{E}-16$ & $4.46 \mathrm{E}-15$ & 0.6601 & 0.2272 & 0.1596 \\
\hline A44 & $2 / 18 / 1999$ & $6.92 \mathrm{E}-17$ & $8.82 \mathrm{E}-18$ & $0.00 \mathrm{E}+00$ & $1.15 \mathrm{E}-15$ & 0.1708 & 0.0000 & 0.3398 \\
\hline A44 & $3 / 16 / 1999$ & $6.10 \mathrm{E}-17$ & $1.01 \mathrm{E}-16$ & $1.42 \mathrm{E}-15$ & $5.34 \mathrm{E}-15$ & 0.7903 & 0.7189 & 0.2995 \\
\hline
\end{tabular}


Table 4. Xenon Tracer Results from 1996 and 1998 Recharge Experiments.

\begin{tabular}{|c|c|c|c|c|c|c|c|c|}
\hline \multirow{2}{*}{ Sample } & \multirow{2}{*}{$\begin{array}{l}\text { Sample } \\
\text { Date }\end{array}$} & \multicolumn{2}{|c|}{${ }^{124} \mathrm{Xe} \quad{ }^{128} \mathrm{Xe}$} & ${ }^{29} \mathrm{Xe}$ & \multirow{2}{*}{${ }^{36} \mathrm{Xe}$} & $\mathrm{C} / \mathrm{Co}{ }^{136} \mathrm{Xe}$ & \multirow{2}{*}{$\begin{array}{l}\mathrm{C} / \mathrm{Co}{ }^{129} \mathrm{Xe} \\
\text { as percent }\end{array}$} & \multirow{2}{*}{$\begin{array}{l}\mathrm{C} / \mathrm{Co}^{124} \mathrm{Xe} \\
\text { as percent }\end{array}$} \\
\hline & & $\mathrm{mol} / \mathrm{mol} \mathrm{H}_{2} \mathrm{O}$ & $5=2$ & 2 & & as percent & & \\
\hline A44 & 4/15/1999 & $6.17 \mathrm{E}-17$ & $4.74 \mathrm{E}-16$ & $1.91 \mathrm{E}-15$ & $1.65 \mathrm{E}-15$ & 0.2436 & 0.9706 & 0.3030 \\
\hline A44 & 7/8/1999 & $7.29 \mathrm{E}-17$ & $4.13 \mathrm{E}-16$ & $2.41 \mathrm{E}-15$ & $1.13 \mathrm{E}-14$ & 1.6763 & 1.2227 & 0.3580 \\
\hline A44 & 9/27/1999 & $6.17 \mathrm{E}-17$ & $3.42 \mathrm{E}-16$ & $8.72 \mathrm{E}-16$ & $0.00 \mathrm{E}+00$ & 0.0000 & 0.4423 & 0.3030 \\
\hline AM10 & $10 / 30 / 1998$ & $2.21 \mathrm{E}-17$ & $2.02 \mathrm{E}-16$ & $1.84 \mathrm{E}-16$ & $1.71 \mathrm{E}-16$ & 0.0253 & 0.0931 & 0.1086 \\
\hline AM10 & $11 / 10 / 1998$ & $3.69 \mathrm{E}-17$ & $2.41 \mathrm{E}-16$ & $0.00 \mathrm{E}+00$ & $1.35 \mathrm{E}-17$ & 0.0020 & 0.0000 & 0.1810 \\
\hline AM10 & 1/5/1999 & $2.18 \mathrm{E}-17$ & $9.62 \mathrm{E}-17$ & $0.00 \mathrm{E}+00$ & $9.00 \mathrm{E}-16$ & 0.1332 & 0.0000 & 0.1072 \\
\hline AM10 & $1 / 20 / 1999$ & $1.90 \mathrm{E}-17$ & $2.02 \mathrm{E}-16$ & $0.00 \mathrm{E}+00$ & $3.14 \mathrm{E}-15$ & 0.4648 & 0.0000 & 0.0932 \\
\hline AM10 & 2/17/1999 & $3.50 \mathrm{E}-17$ & $1.18 \mathrm{E}-16$ & $0.00 \mathrm{E}+00$ & $8.82 \mathrm{E}-15$ & 1.3053 & 0.0000 & 0.1720 \\
\hline AM10 & $3 / 16 / 1999$ & $1.65 \mathrm{E}-17$ & $2.26 \mathrm{E}-16$ & $2.19 \mathrm{E}-16$ & $9.79 \mathrm{E}-15$ & 1.4489 & 0.1109 & 0.0810 \\
\hline AM10 & $3 / 30 / 1999$ & $1.00 \mathrm{E}-17$ & $2.56 \mathrm{E}-16$ & $3.54 \mathrm{E}-15$ & $8.74 \mathrm{E}-15$ & 1.2939 & 1.7966 & 0.0491 \\
\hline AM10 & 4/13/1999 & $3.86 \mathrm{E}-19$ & $3.21 \mathrm{E}-17$ & $1.73 \mathrm{E}-16$ & $8.63 \mathrm{E}-15$ & 1.2769 & 0.0877 & 0.0019 \\
\hline AM10 & $4 / 27 / 1999$ & $1.83 \mathrm{E}-17$ & $1.44 \mathrm{E}-16$ & $7.51 \mathrm{E}-16$ & $7.64 \mathrm{E}-15$ & 1.1298 & 0.3807 & 0.0901 \\
\hline AM10 & 6/8/1999 & $1.01 \mathrm{E}-16$ & $8.72 \mathrm{E}-17$ & $3.68 \mathrm{E}-15$ & $7.67 \mathrm{E}-15$ & 1.1347 & 1.8658 & 0.4962 \\
\hline AM10 & $6 / 25 / 1999$ & $2.29 \mathrm{E}-17$ & $1.23 \mathrm{E}-16$ & $2.86 \mathrm{E}-16$ & $6.43 \mathrm{E}-15$ & 0.9518 & 0.1450 & 0.1126 \\
\hline AM10 & 7/7/1999 & $1.55 \mathrm{E}-17$ & $6.06 \mathrm{E}-16$ & $0.00 \mathrm{E}+00$ & $5.65 \mathrm{E}-15$ & 0.8364 & 0.0000 & 0.0760 \\
\hline AM10 & $7 / 21 / 1999$ & $1.21 \mathrm{E}-16$ & $2.47 \mathrm{E}-16$ & $2.40 \mathrm{E}-15$ & $4.66 \mathrm{E}-15$ & 0.6895 & 1.2169 & 0.5932 \\
\hline AM10 & 8/4/1999 & $1.53 \mathrm{E}-16$ & $2.97 \mathrm{E}-16$ & $3.19 \mathrm{E}-15$ & $3.60 \mathrm{E}-15$ & 0.5320 & 1.6169 & 0.7510 \\
\hline AM10 & $8 / 27 / 1999$ & $1.07 \mathrm{E}-17$ & 4.10E-16 & $0.00 \mathrm{E}+00$ & $0.00 \mathrm{E}+00$ & 0.0000 & 0.0000 & 0.0526 \\
\hline AM10 & $9 / 28 / 1999$ & $3.45 \mathrm{E}-17$ & $1.16 \mathrm{E}-17$ & $1.53 \mathrm{E}-15$ & $1.50 \mathrm{E}-15$ & 0.2216 & 0.7762 & 0.1696 \\
\hline AM10 & 10/27/1999 & $1.10 \mathrm{E}-16$ & $7.52 \mathrm{E}-16$ & $0.00 \mathrm{E}+00$ & $0.00 \mathrm{E}+00$ & 0.0000 & 0.0000 & 0.5420 \\
\hline AM10 & $11 / 30 / 1999$ & $6.09 \mathrm{E}-17$ & $3.57 \mathrm{E}-17$ & $3.33 \mathrm{E}-15$ & $3.38 \mathrm{E}-15$ & 0.5008 & 1.6867 & 0.2988 \\
\hline AM10 & $3 / 22 / 2000$ & $0.00 \mathrm{E}+00$ & $3.68 \mathrm{E}-16$ & $6.92 \mathrm{E}-15$ & $1.32 \mathrm{E}-15$ & 0.1959 & 3.5080 & 0.0000 \\
\hline AM12 & $7 / 21 / 1999$ & $3.38 \mathrm{E}-17$ & $0.00 \mathrm{E}+00$ & $0.00 \mathrm{E}+00$ & $0.00 \mathrm{E}+00$ & 0.0000 & 0.0000 & 0.1659 \\
\hline AM13 & $11 / 9 / 1998$ & $1.08 \mathrm{E}-17$ & $9.84 \mathrm{E}-17$ & $0.00 \mathrm{E}+00$ & $0.00 \mathrm{E}+00$ & 0.0000 & 0.0000 & 0.0528 \\
\hline AM13 & $12 / 22 / 1998$ & $3.65 \mathrm{E}-17$ & $1.12 \mathrm{E}-16$ & $1.51 \mathrm{E}-16$ & $0.00 \mathrm{E}+00$ & 0.0000 & 0.0763 & 0.1794 \\
\hline AM13 & $1 / 18 / 1999$ & $5.08 \mathrm{E}-18$ & $2.08 \mathrm{E}-16$ & 5.93E-17 & $3.34 \mathrm{E}-17$ & 0.0049 & 0.0301 & 0.0249 \\
\hline AM13 & 2/2/1999 & $0.00 \mathrm{E}+00$ & $3.38 \mathrm{E}-17$ & $0.00 \mathrm{E}+00$ & $0.00 \mathrm{E}+00$ & 0.0000 & 0.0000 & 0.0000 \\
\hline AM13 & 2/16/1999 & $2.56 \mathrm{E}-18$ & $9.50 \mathrm{E}-17$ & $5.02 \mathrm{E}-16$ & $0.00 \mathrm{E}+00$ & 0.0000 & 0.2544 & 0.0126 \\
\hline AM13 & $3 / 29 / 1999$ & $2.29 \mathrm{E}-17$ & $5.26 \mathrm{E}-17$ & $2.49 \mathrm{E}-15$ & $0.00 \mathrm{E}+00$ & 0.0000 & 1.2618 & 0.1123 \\
\hline AM13 & 4/13/1999 & $3.26 \mathrm{E}-17$ & $3.28 \mathrm{E}-16$ & $1.06 \mathrm{E}-15$ & $0.00 \mathrm{E}+00$ & 0.0000 & 0.5376 & 0.1602 \\
\hline AM13 & $10 / 10 / 2000$ & $0.00 \mathrm{E}+00$ & $1.98 \mathrm{E}-16$ & $0.00 \mathrm{E}+00$ & $0.00 \mathrm{E}+00$ & 0.0000 & 0.0000 & 0.0000 \\
\hline AM14 & $4 / 30 / 1997$ & $3.12 \mathrm{E}-17$ & $3.90 \mathrm{E}-16$ & $3.94 \mathrm{E}-18$ & $0.00 \mathrm{E}+00$ & 0.0000 & 0.0020 & 0.1531 \\
\hline AM14 & $10 / 29 / 1998$ & $2.30 \mathrm{E}-17$ & $0.00 \mathrm{E}+00$ & $0.00 \mathrm{E}+00$ & $0.00 \mathrm{E}+00$ & 0.0000 & 0.0000 & 0.1131 \\
\hline AM14 & $11 / 23 / 1998$ & $3.34 \mathrm{E}-17$ & $0.00 \mathrm{E}+00$ & $3.46 \mathrm{E}-16$ & $0.00 \mathrm{E}+00$ & 0.0000 & 0.1753 & 0.1642 \\
\hline AM14 & $12 / 22 / 1998$ & $3.29 \mathrm{E}-17$ & $0.00 \mathrm{E}+00$ & $2.82 \mathrm{E}-16$ & $0.00 \mathrm{E}+00$ & 0.0000 & 0.1432 & 0.1617 \\
\hline AM14 & $1 / 17 / 1999$ & $4.60 \mathrm{E}-17$ & $0.00 \mathrm{E}+00$ & $0.00 \mathrm{E}+00$ & $0.00 \mathrm{E}+00$ & 0.0000 & 0.0000 & 0.2257 \\
\hline AM14 & 2/18/1999 & $5.70 \mathrm{E}-17$ & $2.25 \mathrm{E}-16$ & $5.66 \mathrm{E}-16$ & $0.00 \mathrm{E}+00$ & 0.0000 & 0.2869 & 0.2800 \\
\hline AM14 & $3 / 16 / 1999$ & $4.54 \mathrm{E}-17$ & $0.00 \mathrm{E}+00$ & $3.27 \mathrm{E}-16$ & $0.00 \mathrm{E}+00$ & 0.0000 & 0.1658 & 0.2229 \\
\hline AM14 & 4/13/1999 & $1.01 \mathrm{E}-18$ & $1.44 \mathrm{E}-16$ & $0.00 \mathrm{E}+00$ & $0.00 \mathrm{E}+00$ & 0.0000 & 0.0000 & 0.0050 \\
\hline AM14 & $5 / 11 / 1999$ & $0.00 \mathrm{E}+00$ & $0.00 \mathrm{E}+00$ & $2.26 \mathrm{E}-15$ & $0.00 \mathrm{E}+00$ & 0.0000 & 1.1456 & 0.0000 \\
\hline AM14 & 7/7/1999 & $3.78 \mathrm{E}-17$ & $1.83 \mathrm{E}-16$ & $4.84 \mathrm{E}-16$ & $0.00 \mathrm{E}+00$ & 0.0000 & 0.2456 & 0.1855 \\
\hline AM14 & 8/2/1999 & $2.03 \mathrm{E}-17$ & $4.59 \mathrm{E}-16$ & $2.14 \mathrm{E}-15$ & $0.00 \mathrm{E}+00$ & 0.0000 & 1.0844 & 0.0996 \\
\hline AM14 & 9/3/1999 & $4.22 \mathrm{E}-17$ & $1.70 \mathrm{E}-16$ & $2.78 \mathrm{E}-16$ & $6.43 \mathrm{E}-15$ & 0.9518 & 0.1409 & 0.2073 \\
\hline AM14 & $9 / 28 / 1999$ & $0.00 \mathrm{E}+00$ & $0.00 \mathrm{E}+00$ & $0.00 \mathrm{E}+00$ & $0.00 \mathrm{E}+00$ & 0.0000 & 0.0000 & 0.0000 \\
\hline AM14 & $10 / 26 / 1999$ & $4.89 \mathrm{E}-17$ & $1.08 \mathrm{E}-16$ & $0.00 \mathrm{E}+00$ & $5.50 \mathrm{E}-15$ & 0.8137 & 0.0000 & 0.2401 \\
\hline AM14 & $1 / 24 / 2000$ & $1.48 \mathrm{E}-16$ & $9.45 \mathrm{E}-17$ & $6.22 \mathrm{E}-16$ & $5.65 \mathrm{E}-15$ & 0.8368 & 0.3153 & 0.7254 \\
\hline AM14 & $3 / 25 / 2000$ & $0.00 \mathrm{E}+00$ & $0.00 \mathrm{E}+00$ & $0.00 \mathrm{E}+00$ & $7.09 \mathrm{E}-15$ & 1.0492 & 0.0000 & 0.0000 \\
\hline AM14 & $5 / 17 / 2000$ & $2.60 \mathrm{E}-17$ & $9.30 \mathrm{E}-16$ & $9.61 \mathrm{E}-15$ & $6.75 \mathrm{E}-15$ & 0.9989 & 4.8707 & 0.1275 \\
\hline
\end{tabular}


Table 4. Xenon Tracer Results from 1996 and 1998 Recharge Experiments.

\begin{tabular}{|c|c|c|c|c|c|c|c|c|}
\hline \multirow[t]{2}{*}{ Sample } & \multirow{2}{*}{$\begin{array}{l}\text { Sample } \\
\text { Date }\end{array}$} & \multicolumn{2}{|c|}{${ }^{124} \mathrm{Xe} \quad{ }^{128} \mathrm{Xe}$} & ${ }^{29} \mathrm{Xe}$ & \multirow{2}{*}{${ }^{136} \mathrm{Xe}$} & $\mathrm{C} / \mathrm{Co}{ }^{136} \mathrm{Xe}$ & \multirow{2}{*}{$\begin{array}{l}\mathrm{C} / \mathrm{Co}{ }^{129} \mathrm{Xe} \\
\text { as percent }\end{array}$} & \multirow{2}{*}{$\begin{array}{l}\mathrm{C} / \mathrm{Co}^{124} \mathrm{Xe} \\
\text { as percent }\end{array}$} \\
\hline & & $\mathrm{mol} / \mathrm{mol} \mathrm{H}_{2}$ & $5=2$ & 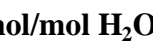 & & as percent & & \\
\hline AM14 & $7 / 15 / 2000$ & $4.56 \mathrm{E}-17$ & $1.29 \mathrm{E}-16$ & $0.00 \mathrm{E}+00$ & $0.00 \mathrm{E}+00$ & 0.0000 & 0.0000 & 0.2242 \\
\hline AM14 & $10 / 18 / 2000$ & $0.00 \mathrm{E}+00$ & $2.38 \mathrm{E}-16$ & $1.10 \mathrm{E}-15$ & $1.74 \mathrm{E}-15$ & 0.2574 & 0.5563 & 0.0000 \\
\hline AM15 & $7 / 15 / 2000$ & $0.00 \mathrm{E}+00$ & $3.14 \mathrm{E}-17$ & $0.00 \mathrm{E}+00$ & $4.56 \mathrm{E}-15$ & 0.6741 & 0.0000 & 0.0000 \\
\hline AM15 & $10 / 9 / 2000$ & $4.41 \mathrm{E}-17$ & $0.00 \mathrm{E}+00$ & $0.00 \mathrm{E}+00$ & $5.14 \mathrm{E}-15$ & 0.7605 & 0.0000 & 0.2163 \\
\hline AM23 & $10 / 18 / 2000$ & $1.28 \mathrm{E}-17$ & $1.18 \mathrm{E}-16$ & $1.35 \mathrm{E}-15$ & $3.80 \mathrm{E}-15$ & 0.5628 & 0.6849 & 0.0627 \\
\hline AM29 & $5 / 17 / 2000$ & $5.28 \mathrm{E}-17$ & $0.00 \mathrm{E}+00$ & $0.00 \mathrm{E}+00$ & $7.60 \mathrm{E}-16$ & 0.1125 & 0.0000 & 0.2592 \\
\hline AM29 & $7 / 15 / 2000$ & $8.71 \mathrm{E}-18$ & $2.18 \mathrm{E}-16$ & $4.15 \mathrm{E}-16$ & $9.57 \mathrm{E}-15$ & 1.4161 & 0.2107 & 0.0428 \\
\hline AM29 & $10 / 9 / 2000$ & $0.00 \mathrm{E}+00$ & $2.94 \mathrm{E}-16$ & $3.60 \mathrm{E}-17$ & $6.56 \mathrm{E}-15$ & 0.9707 & 0.0182 & 0.0000 \\
\hline AM44 & $12 / 4 / 1996$ & $1.67 \mathrm{E}-16$ & $2.20 \mathrm{E}-16$ & $0.00 \mathrm{E}+00$ & $1.55 \mathrm{E}-16$ & 0.0230 & 0.0000 & 0.8221 \\
\hline AM44 & $12 / 30 / 1996$ & $6.87 \mathrm{E}-16$ & $6.97 \mathrm{E}-17$ & $6.64 \mathrm{E}-16$ & $0.00 \mathrm{E}+00$ & 0.0000 & 0.3366 & 3.3730 \\
\hline AM44 & $1 / 27 / 1997$ & $8.92 \mathrm{E}-15$ & $8.46 \mathrm{E}-16$ & $5.78 \mathrm{E}-15$ & $0.00 \mathrm{E}+00$ & 0.0000 & 2.9294 & 43.8020 \\
\hline AM44 & $2 / 21 / 1997$ & $1.41 \mathrm{E}-15$ & $7.58 \mathrm{E}-17$ & $6.45 \mathrm{E}-16$ & $0.00 \mathrm{E}+00$ & 0.0000 & 0.3269 & 6.9300 \\
\hline AM44 & $3 / 31 / 1997$ & $4.90 \mathrm{E}-16$ & $5.20 \mathrm{E}-17$ & $0.00 \mathrm{E}+00$ & $0.00 \mathrm{E}+00$ & 0.0000 & 0.0000 & 2.4044 \\
\hline AM44 & $10 / 8 / 1998$ & $0.00 \mathrm{E}+00$ & $2.36 \mathrm{E}-18$ & $0.00 \mathrm{E}+00$ & $0.00 \mathrm{E}+00$ & 0.0000 & 0.0000 & 0.0000 \\
\hline AM44 & $10 / 21 / 1998$ & $1.05 \mathrm{E}-16$ & $2.12 \mathrm{E}-15$ & $1.38 \mathrm{E}-14$ & $0.00 \mathrm{E}+00$ & 0.0000 & 7.0048 & 0.5135 \\
\hline AM44 & $10 / 28 / 1998$ & $3.16 \mathrm{E}-17$ & $4.50 \mathrm{E}-16$ & $1.43 \mathrm{E}-15$ & $0.00 \mathrm{E}+00$ & 0.0000 & 0.7237 & 0.1550 \\
\hline AM44 & $11 / 4 / 1998$ & $0.00 \mathrm{E}+00$ & $3.68 \mathrm{E}-16$ & $0.00 \mathrm{E}+00$ & $0.00 \mathrm{E}+00$ & 0.0000 & 0.0000 & 0.0000 \\
\hline AM44 & $11 / 9 / 1998$ & $0.00 \mathrm{E}+00$ & $1.34 \mathrm{E}-16$ & $0.00 \mathrm{E}+00$ & $2.80 \mathrm{E}-17$ & 0.0041 & 0.0000 & 0.0000 \\
\hline AM44 & $11 / 17 / 1998$ & $4.97 \mathrm{E}-17$ & $2.84 \mathrm{E}-16$ & $1.86 \mathrm{E}-15$ & $0.00 \mathrm{E}+00$ & 0.0000 & 0.9428 & 0.2443 \\
\hline AM44 & $11 / 25 / 1998$ & $1.79 \mathrm{E}-17$ & $1.21 \mathrm{E}-15$ & $5.18 \mathrm{E}-15$ & $1.28 \mathrm{E}-16$ & 0.0190 & 2.6279 & 0.0877 \\
\hline AM44 & $12 / 7 / 1998$ & $1.35 \mathrm{E}-16$ & $5.40 \mathrm{E}-15$ & $2.79 \mathrm{E}-14$ & $0.00 \mathrm{E}+00$ & 0.0000 & 14.1260 & 0.6616 \\
\hline AM44 & $12 / 30 / 1998$ & $5.03 \mathrm{E}-16$ & $2.66 \mathrm{E}-14$ & $1.39 \mathrm{E}-13$ & $0.00 \mathrm{E}+00$ & 0.0000 & 70.2400 & 2.4711 \\
\hline AM44 & 1/5/1999 & $3.16 \mathrm{E}-16$ & $1.45 \mathrm{E}-14$ & $7.67 \mathrm{E}-14$ & $0.00 \mathrm{E}+00$ & 0.0000 & 38.8690 & 1.5515 \\
\hline AM44 & $1 / 12 / 1999$ & $3.00 \mathrm{E}-16$ & $1.30 \mathrm{E}-14$ & $6.80 \mathrm{E}-14$ & $0.00 \mathrm{E}+00$ & 0.0000 & 34.4960 & 1.4729 \\
\hline AM44 & $1 / 19 / 1999$ & $8.71 \mathrm{E}-17$ & $9.81 \mathrm{E}-17$ & $5.66 \mathrm{E}-16$ & $0.00 \mathrm{E}+00$ & 0.0000 & 0.2870 & 0.4278 \\
\hline AM44 & $1 / 25 / 1999$ & $1.78 \mathrm{E}-16$ & $8.62 \mathrm{E}-15$ & $4.46 \mathrm{E}-14$ & $0.00 \mathrm{E}+00$ & 0.0000 & 22.6380 & 0.8738 \\
\hline AM44 & 2/4/1999 & $1.75 \mathrm{E}-16$ & $8.24 \mathrm{E}-15$ & $3.91 \mathrm{E}-14$ & $0.00 \mathrm{E}+00$ & 0.0000 & 19.8050 & 0.8608 \\
\hline AM44 & 2/18/1999 & $6.97 \mathrm{E}-17$ & $1.95 \mathrm{E}-15$ & $1.05 \mathrm{E}-14$ & $0.00 \mathrm{E}+00$ & 0.0000 & 5.3433 & 0.3421 \\
\hline AM44 & 3/3/1999 & 4.24E-17 & $1.84 \mathrm{E}-15$ & $5.45 \mathrm{E}-15$ & $0.00 \mathrm{E}+00$ & 0.0000 & 2.7634 & 0.2081 \\
\hline AM44 & $5 / 12 / 1999$ & $1.66 \mathrm{E}-17$ & $2.85 \mathrm{E}-16$ & $1.83 \mathrm{E}-15$ & $1.96 \mathrm{E}-15$ & 0.2898 & 0.9289 & 0.0817 \\
\hline AM44 & $6 / 10 / 1999$ & $5.24 \mathrm{E}-17$ & $3.32 \mathrm{E}-16$ & $2.68 \mathrm{E}-15$ & $0.00 \mathrm{E}+00$ & 0.0000 & 1.3606 & 0.2574 \\
\hline AM44 & 9/10/1999 & $5.66 \mathrm{E}-17$ & $3.01 \mathrm{E}-16$ & $3.10 \mathrm{E}-15$ & $0.00 \mathrm{E}+00$ & 0.0000 & 1.5718 & 0.2778 \\
\hline AM6 & $12 / 23 / 1998$ & $0.00 \mathrm{E}+00$ & $1.71 \mathrm{E}-16$ & $0.00 \mathrm{E}+00$ & $0.00 \mathrm{E}+00$ & 0.0000 & 0.0000 & 0.0000 \\
\hline AM6 & $1 / 18 / 1999$ & $3.08 \mathrm{E}-17$ & $0.00 \mathrm{E}+00$ & $0.00 \mathrm{E}+00$ & $0.00 \mathrm{E}+00$ & 0.0000 & 0.0000 & 0.1515 \\
\hline AM6 & 2/16/1999 & $1.09 \mathrm{E}-17$ & $5.72 \mathrm{E}-17$ & $1.67 \mathrm{E}-16$ & $0.00 \mathrm{E}+00$ & 0.0000 & 0.0847 & 0.0537 \\
\hline AM6 & 4/12/1999 & $0.00 \mathrm{E}+00$ & $5.71 \mathrm{E}-17$ & $1.72 \mathrm{E}-16$ & $2.62 \mathrm{E}-16$ & 0.0388 & 0.0871 & 0.0000 \\
\hline AM7 & $10 / 16 / 1998$ & $5.80 \mathrm{E}-18$ & $6.59 \mathrm{E}-19$ & $1.43 \mathrm{E}-16$ & $5.75 \mathrm{E}-16$ & 0.0851 & 0.0727 & 0.0285 \\
\hline AM7 & $10 / 22 / 1998$ & $0.00 \mathrm{E}+00$ & $6.82 \mathrm{E}-19$ & $0.00 \mathrm{E}+00$ & $1.40 \mathrm{E}-15$ & 0.2075 & 0.0000 & 0.0000 \\
\hline AM7 & $11 / 10 / 1998$ & $0.00 \mathrm{E}+00$ & $0.00 \mathrm{E}+00$ & $0.00 \mathrm{E}+00$ & 7.39E-18 & 0.0011 & 0.0000 & 0.0000 \\
\hline AM7 & $11 / 20 / 1998$ & $3.71 \mathrm{E}-17$ & $9.24 \mathrm{E}-17$ & $6.15 \mathrm{E}-16$ & $1.03 \mathrm{E}-16$ & 0.0153 & 0.3121 & 0.1822 \\
\hline AM7 & $12 / 4 / 1998$ & $0.00 \mathrm{E}+00$ & $0.00 \mathrm{E}+00$ & $6.44 \mathrm{E}-16$ & $5.15 \mathrm{E}-15$ & 0.7618 & 0.3263 & 0.0000 \\
\hline AM7 & $12 / 10 / 1998$ & $2.07 \mathrm{E}-17$ & $1.21 \mathrm{E}-16$ & $3.38 \mathrm{E}-16$ & $1.28 \mathrm{E}-14$ & 1.9010 & 0.1713 & 0.1017 \\
\hline AM7 & $12 / 27 / 1998$ & $7.84 \mathrm{E}-18$ & $9.69 \mathrm{E}-17$ & $0.00 \mathrm{E}+00$ & $2.25 \mathrm{E}-14$ & 3.3302 & 0.0000 & 0.0385 \\
\hline AM7 & 1/6/1999 & $7.60 \mathrm{E}-18$ & $3.12 \mathrm{E}-17$ & $0.00 \mathrm{E}+00$ & $3.90 \mathrm{E}-14$ & 5.7661 & 0.0000 & 0.0373 \\
\hline AM7 & $1 / 12 / 1999$ & $0.00 \mathrm{E}+00$ & $8.80 \mathrm{E}-17$ & $0.00 \mathrm{E}+00$ & $5.69 \mathrm{E}-14$ & 8.4185 & 0.0000 & 0.0000 \\
\hline AM7 & $1 / 20 / 1999$ & $0.00 \mathrm{E}+00$ & $1.49 \mathrm{E}-16$ & $0.00 \mathrm{E}+00$ & $6.05 \mathrm{E}-14$ & 8.9497 & 0.0000 & 0.0000 \\
\hline
\end{tabular}


Table 4. Xenon Tracer Results from 1996 and 1998 Recharge Experiments.

\begin{tabular}{|c|c|c|c|c|c|c|c|c|}
\hline Sample & $\begin{array}{l}\text { Sample } \\
\text { Date }\end{array}$ & $\begin{array}{l}{ }^{124} \mathrm{Xe} \\
\mathrm{mol} / \mathrm{mol} \mathrm{H}_{2} \mathrm{O}\end{array}$ & $\begin{array}{l}\mathrm{Xe} \\
\mathrm{l} / \mathrm{mol} \mathrm{H} \mathrm{H}_{2} \mathrm{O}\end{array}$ & $\begin{array}{l}\mathrm{Xe} \\
\mathrm{ol} / \mathrm{mol} \mathrm{H}_{2} \mathrm{O}\end{array}$ & & $\begin{array}{l}{ }^{{ }^{136}} \mathrm{Xe} \\
\text { ercent }\end{array}$ & $\begin{array}{l}\mathrm{C} / \mathrm{Co}{ }^{129} \mathrm{Xe} \\
\text { as percent }\end{array}$ & $\begin{array}{l}\mathrm{C} / \mathrm{Co}^{124} \mathrm{Xe} \\
\text { as percent }\end{array}$ \\
\hline AM7 & $1 / 29 / 1999$ & $3.84 \mathrm{E}-17$ & $6.99 \mathrm{E}-16$ & $1.77 \mathrm{E}-15$ & $3.57 \mathrm{E}-14$ & 5.2886 & 0.8959 & 0.1886 \\
\hline AM7 & 2/18/1999 & $2.44 \mathrm{E}-18$ & $1.18 \mathrm{E}-16$ & $2.60 \mathrm{E}-16$ & $1.65 \mathrm{E}-14$ & 2.4468 & 0.1317 & 0.0120 \\
\hline AM7 & 3/4/1999 & $1.51 \mathrm{E}-17$ & $1.42 \mathrm{E}-16$ & $1.77 \mathrm{E}-16$ & $8.56 \mathrm{E}-15$ & 1.2674 & 0.0900 & 0.0739 \\
\hline AM7 & $3 / 30 / 1999$ & $4.19 \mathrm{E}-17$ & $2.77 \mathrm{E}-16$ & $6.25 \mathrm{E}-15$ & $8.31 \mathrm{E}-16$ & 0.1230 & 3.1714 & 0.2058 \\
\hline AM7 & 4/13/1999 & $2.88 \mathrm{E}-18$ & $4.26 \mathrm{E}-16$ & $3.79 \mathrm{E}-17$ & $2.45 \mathrm{E}-15$ & 0.3624 & 0.0192 & 0.0141 \\
\hline AM8 & $10 / 30 / 1998$ & $7.19 \mathrm{E}-18$ & $2.21 \mathrm{E}-18$ & $0.00 \mathrm{E}+00$ & $5.77 \mathrm{E}-17$ & 0.0085 & 0.0000 & 0.0353 \\
\hline AM8 & $11 / 23 / 1998$ & $4.71 \mathrm{E}-18$ & $2.20 \mathrm{E}-16$ & $0.00 \mathrm{E}+00$ & $0.00 \mathrm{E}+00$ & 0.0000 & 0.0000 & 0.0231 \\
\hline AM8 & $12 / 23 / 1998$ & $1.96 \mathrm{E}-17$ & $5.60 \mathrm{E}-17$ & $0.00 \mathrm{E}+00$ & $3.89 \mathrm{E}-16$ & 0.0576 & 0.0000 & 0.0964 \\
\hline AM8 & $1 / 18 / 1999$ & $6.30 \mathrm{E}-18$ & $1.08 \mathrm{E}-16$ & $0.00 \mathrm{E}+00$ & $1.42 \mathrm{E}-15$ & 0.2105 & 0.0000 & 0.0309 \\
\hline AM8 & $1 / 18 / 1999$ & $1.50 \mathrm{E}-17$ & $9.17 \mathrm{E}-17$ & $4.69 \mathrm{E}-16$ & $8.16 \mathrm{E}-16$ & 0.1208 & 0.2379 & 0.0735 \\
\hline AM8 & 2/16/1999 & $0.00 \mathrm{E}+00$ & $4.05 \mathrm{E}-17$ & $6.41 \mathrm{E}-17$ & $1.24 \mathrm{E}-14$ & 1.8417 & 0.0325 & 0.0000 \\
\hline AM8 & $3 / 16 / 1999$ & $1.34 \mathrm{E}-17$ & $2.29 \mathrm{E}-16$ & $3.45 \mathrm{E}-15$ & $1.95 \mathrm{E}-14$ & 2.8854 & 1.7471 & 0.0659 \\
\hline AM8 & 4/12/1999 & $0.00 \mathrm{E}+00$ & $1.87 \mathrm{E}-17$ & $2.62 \mathrm{E}-17$ & $1.84 \mathrm{E}-14$ & 2.7161 & 0.0133 & 0.0000 \\
\hline AM8 & $5 / 10 / 1999$ & $7.94 \mathrm{E}-18$ & $0.00 \mathrm{E}+00$ & $0.00 \mathrm{E}+00$ & $1.72 \mathrm{E}-14$ & 2.5429 & 0.0000 & 0.0390 \\
\hline AM8 & 9/3/1999 & $2.18 \mathrm{E}-18$ & $0.00 \mathrm{E}+00$ & $0.00 \mathrm{E}+00$ & $1.03 \mathrm{E}-14$ & 1.5302 & 0.0000 & 0.0107 \\
\hline AM8 & 11/30/1999 & $0.00 \mathrm{E}+00$ & $0.00 \mathrm{E}+00$ & $0.00 \mathrm{E}+00$ & $2.85 \mathrm{E}-15$ & 0.4224 & 0.0000 & 0.0000 \\
\hline AM8 & $5 / 19 / 2000$ & $1.16 \mathrm{E}-17$ & $1.65 \mathrm{E}-16$ & $0.00 \mathrm{E}+00$ & $1.01 \mathrm{E}-15$ & 0.1495 & 0.0000 & 0.0571 \\
\hline AM8 & $7 / 15 / 2000$ & $4.71 \mathrm{E}-17$ & $6.85 \mathrm{E}-16$ & $0.00 \mathrm{E}+00$ & $0.00 \mathrm{E}+00$ & 0.0000 & 0.0000 & 0.2313 \\
\hline AM9 & $4 / 2 / 1997$ & $0.00 \mathrm{E}+00$ & $6.17 \mathrm{E}-16$ & $0.00 \mathrm{E}+00$ & $4.80 \mathrm{E}-16$ & 0.0710 & 0.0000 & 0.0000 \\
\hline AM9 & $4 / 21 / 1997$ & $0.00 \mathrm{E}+00$ & $8.50 \mathrm{E}-16$ & $0.00 \mathrm{E}+00$ & $3.46 \mathrm{E}-16$ & 0.0513 & 0.0000 & 0.0000 \\
\hline AM9 & $10 / 30 / 1998$ & $1.91 \mathrm{E}-17$ & $1.88 \mathrm{E}-16$ & $0.00 \mathrm{E}+00$ & $1.43 \mathrm{E}-16$ & 0.0212 & 0.0000 & 0.0938 \\
\hline AM9 & $3 / 20 / 1999$ & $4.24 \mathrm{E}-17$ & $3.36 \mathrm{E}-16$ & $4.52 \mathrm{E}-15$ & $0.00 \mathrm{E}+00$ & 0.0000 & 2.2930 & 0.2083 \\
\hline AM9 & $4 / 17 / 1999$ & $2.18 \mathrm{E}-17$ & $1.45 \mathrm{E}-16$ & $1.11 \mathrm{E}-15$ & $3.25 \mathrm{E}-15$ & 0.4810 & 0.5630 & 0.1069 \\
\hline AM9 & $6 / 13 / 1999$ & $7.08 \mathrm{E}-17$ & $1.91 \mathrm{E}-16$ & $2.77 \mathrm{E}-15$ & $5.14 \mathrm{E}-15$ & 0.7601 & 1.4038 & 0.3479 \\
\hline AM9 & $6 / 15 / 1999$ & $1.73 \mathrm{E}-18$ & $5.56 \mathrm{E}-16$ & $2.71 \mathrm{E}-15$ & $1.50 \mathrm{E}-15$ & 0.2212 & 1.3741 & 0.0085 \\
\hline AM9 & 7/11/1999 & $4.64 \mathrm{E}-18$ & $2.19 \mathrm{E}-16$ & $8.13 \mathrm{E}-16$ & $1.20 \mathrm{E}-14$ & 1.7693 & 0.4120 & 0.0228 \\
\hline AM9 & $8 / 29 / 1999$ & $0.00 \mathrm{E}+00$ & 4.19E-17 & $0.00 \mathrm{E}+00$ & $1.19 \mathrm{E}-14$ & 1.7549 & 0.0000 & 0.0000 \\
\hline AM9 & $12 / 5 / 1999$ & $0.00 \mathrm{E}+00$ & $2.24 \mathrm{E}-16$ & $0.00 \mathrm{E}+00$ & $5.57 \mathrm{E}-15$ & 0.8241 & 0.0000 & 0.0000 \\
\hline AM9 & $5 / 14 / 2000$ & $2.33 \mathrm{E}-17$ & $0.00 \mathrm{E}+00$ & $0.00 \mathrm{E}+00$ & $0.00 \mathrm{E}+00$ & 0.0000 & 0.0000 & 0.1146 \\
\hline AMD1/1 & $12 / 22 / 1998$ & $3.25 \mathrm{E}-17$ & $0.00 \mathrm{E}+00$ & $0.00 \mathrm{E}+00$ & $0.00 \mathrm{E}+00$ & 0.0000 & 0.0000 & 0.1596 \\
\hline AMD1/2 & $11 / 9 / 1998$ & $4.12 \mathrm{E}-17$ & $5.17 \mathrm{E}-18$ & $0.00 \mathrm{E}+00$ & 4.97E-17 & 0.0074 & 0.0000 & 0.2024 \\
\hline AMD1/2 & $12 / 22 / 1998$ & $1.76 \mathrm{E}-17$ & $0.00 \mathrm{E}+00$ & $3.49 \mathrm{E}-16$ & $0.00 \mathrm{E}+00$ & 0.0000 & 0.1772 & 0.0864 \\
\hline AMD1/2 & 1/6/1999 & $2.65 \mathrm{E}-17$ & $0.00 \mathrm{E}+00$ & $0.00 \mathrm{E}+00$ & $6.69 \mathrm{E}-17$ & 0.0099 & 0.0000 & 0.1303 \\
\hline AMD1/2 & 2/1/1999 & $5.47 \mathrm{E}-17$ & $3.18 \mathrm{E}-16$ & $3.13 \mathrm{E}-16$ & $0.00 \mathrm{E}+00$ & 0.0000 & 0.1587 & 0.2686 \\
\hline AMD1/2 & 2/17/1999 & $2.50 \mathrm{E}-17$ & $9.83 \mathrm{E}-17$ & $0.00 \mathrm{E}+00$ & $4.48 \mathrm{E}-17$ & 0.0066 & 0.0000 & 0.1228 \\
\hline $\mathrm{AMD} 1 / 2$ & $3 / 1 / 1999$ & $3.25 \mathrm{E}-17$ & $0.00 \mathrm{E}+00$ & $1.74 \mathrm{E}-15$ & $0.00 \mathrm{E}+00$ & 0.0000 & 0.8810 & 0.1597 \\
\hline AMD1/3 & $12 / 9 / 1998$ & $3.20 \mathrm{E}-17$ & $0.00 \mathrm{E}+00$ & $8.01 \mathrm{E}-16$ & $0.00 \mathrm{E}+00$ & 0.0000 & 0.4061 & 0.1572 \\
\hline $\mathrm{AMD} 1 / 3$ & $12 / 22 / 1998$ & $7.44 \mathrm{E}-17$ & $4.66 \mathrm{E}-17$ & $0.00 \mathrm{E}+00$ & $0.00 \mathrm{E}+00$ & 0.0000 & 0.0000 & 0.3653 \\
\hline AMD1/3 & 1/6/1999 & $7.37 \mathrm{E}-17$ & $4.92 \mathrm{E}-17$ & $0.00 \mathrm{E}+00$ & $0.00 \mathrm{E}+00$ & 0.0000 & 0.0000 & 0.3621 \\
\hline AMD1/3 & 2/17/1999 & $2.71 \mathrm{E}-17$ & $1.48 \mathrm{E}-16$ & $1.27 \mathrm{E}-17$ & $0.00 \mathrm{E}+00$ & 0.0000 & 0.0064 & 0.1329 \\
\hline $\mathrm{AMD} 1 / 3$ & 3/1/1999 & $8.90 \mathrm{E}-17$ & $2.10 \mathrm{E}-17$ & $1.81 \mathrm{E}-15$ & $0.00 \mathrm{E}+00$ & 0.0000 & 0.9180 & 0.4371 \\
\hline AMD1/4 & $12 / 22 / 1998$ & $4.12 \mathrm{E}-17$ & $0.00 \mathrm{E}+00$ & $1.08 \mathrm{E}-15$ & $0.00 \mathrm{E}+00$ & 0.0000 & 0.5492 & 0.2023 \\
\hline AMD1/4 & 1/6/1999 & $5.78 \mathrm{E}-17$ & $0.00 \mathrm{E}+00$ & $0.00 \mathrm{E}+00$ & $2.28 \mathrm{E}-16$ & 0.0338 & 0.0000 & 0.2837 \\
\hline AMD1/4 & 2/17/1999 & $5.68 \mathrm{E}-17$ & $2.00 \mathrm{E}-16$ & $2.88 \mathrm{E}-16$ & $1.48 \mathrm{E}-16$ & 0.0219 & 0.1463 & 0.2791 \\
\hline AMD1/4 & 3/1/1999 & $7.58 \mathrm{E}-17$ & $3.03 \mathrm{E}-17$ & $0.00 \mathrm{E}+00$ & $6.00 \mathrm{E}-18$ & 0.0009 & 0.0000 & 0.3725 \\
\hline
\end{tabular}


Table 4. Xenon Tracer Results from 1996 and 1998 Recharge Experiments.

\begin{tabular}{|c|c|c|c|c|c|c|c|c|}
\hline \multirow[t]{2}{*}{ Sample } & \multirow{2}{*}{$\begin{array}{l}\text { Sample } \\
\text { Date }\end{array}$} & \multirow{2}{*}{\multicolumn{2}{|c|}{${ }^{128} \mathrm{Xe}$}} & & \multicolumn{2}{|c|}{${ }^{136} \mathrm{Xe} \quad \mathrm{C} / \mathrm{Co}^{136} \mathrm{Xe}$} & \multirow{2}{*}{$\begin{array}{l}\mathrm{C} / \mathrm{Co}{ }^{129} \mathrm{Xe} \\
\text { as percent }\end{array}$} & \multirow{2}{*}{$\begin{array}{l}\mathrm{C} / \mathrm{Co}^{124} \mathrm{Xe} \\
\text { as percent }\end{array}$} \\
\hline & & & & $\mathrm{l} / \mathrm{mol} \mathrm{H} \mathrm{H}_{2} \mathrm{O}$ & $\mathrm{mol} / \mathrm{mol} \mathrm{H}_{2} \mathrm{O}$ & percent & & \\
\hline AMD1/5 & 2/1/1999 & $3.29 \mathrm{E}-17$ & $1.75 \mathrm{E}-17$ & $1.25 \mathrm{E}-16$ & $1.60 \mathrm{E}-16$ & 0.0236 & 0.0634 & 0.1615 \\
\hline AMD1/5 & 2/17/1999 & $6.39 \mathrm{E}-18$ & $0.00 \mathrm{E}+00$ & $0.00 \mathrm{E}+00$ & $9.49 \mathrm{E}-17$ & 0.0140 & 0.0000 & 0.0314 \\
\hline AMD1/5 & 3/1/1999 & $6.47 \mathrm{E}-18$ & $0.00 \mathrm{E}+00$ & $1.92 \mathrm{E}-16$ & $1.55 \mathrm{E}-16$ & 0.0229 & 0.0975 & 0.0318 \\
\hline AMD1/6 & $2 / 17 / 1999$ & $1.81 \mathrm{E}-17$ & $0.00 \mathrm{E}+00$ & $0.00 \mathrm{E}+00$ & $9.49 \mathrm{E}-17$ & 0.0140 & 0.0000 & 0.0888 \\
\hline AMD1/6 & 3/1/1999 & $0.00 \mathrm{E}+00$ & $0.00 \mathrm{E}+00$ & $0.00 \mathrm{E}+00$ & $0.00 \mathrm{E}+00$ & 0.0000 & 0.0000 & 0.0000 \\
\hline AMD10/1 & 3/3/1998 & $0.00 \mathrm{E}+00$ & $5.04 \mathrm{E}-16$ & $0.00 \mathrm{E}+00$ & $0.00 \mathrm{E}+00$ & 0.0000 & 0.0000 & 0.0000 \\
\hline AMD10/1 & $10 / 28 / 1998$ & $2.53 \mathrm{E}-17$ & $1.11 \mathrm{E}-16$ & $0.00 \mathrm{E}+00$ & $1.58 \mathrm{E}-16$ & 0.0234 & 0.0000 & 0.1243 \\
\hline AMD10/1 & $11 / 9 / 1998$ & $0.00 \mathrm{E}+00$ & $7.78 \mathrm{E}-17$ & $0.00 \mathrm{E}+00$ & $1.42 \mathrm{E}-15$ & 0.2104 & 0.0000 & 0.0000 \\
\hline AMD10/1 & $12 / 8 / 1998$ & $9.27 \mathrm{E}-18$ & $4.01 \mathrm{E}-16$ & $8.88 \mathrm{E}-16$ & $9.75 \mathrm{E}-15$ & 1.4422 & 0.4504 & 0.0455 \\
\hline AMD10/1 & $12 / 21 / 1998$ & $3.18 \mathrm{E}-17$ & 8.67E-16 & $3.73 \mathrm{E}-15$ & $2.05 \mathrm{E}-14$ & 3.0386 & 1.8920 & 0.1560 \\
\hline AMD10/1 & 1/5/1999 & $1.91 \mathrm{E}-17$ & $1.77 \mathrm{E}-15$ & $7.77 \mathrm{E}-15$ & $2.00 \mathrm{E}-14$ & 2.9565 & 3.9391 & 0.0940 \\
\hline AMD10/1 & $1 / 18 / 1999$ & $4.62 \mathrm{E}-17$ & $2.59 \mathrm{E}-15$ & $1.30 \mathrm{E}-14$ & $1.67 \mathrm{E}-14$ & 2.4718 & 6.5672 & 0.2267 \\
\hline AMD10/1 & 2/1/1999 & $6.15 \mathrm{E}-17$ & $2.67 \mathrm{E}-15$ & $1.28 \mathrm{E}-14$ & $1.28 \mathrm{E}-14$ & 1.8934 & 6.4726 & 0.3021 \\
\hline AMD10/1 & $2 / 16 / 1999$ & $4.94 \mathrm{E}-17$ & $3.52 \mathrm{E}-15$ & $1.82 \mathrm{E}-14$ & $8.93 \mathrm{E}-15$ & 1.3214 & 9.2315 & 0.2425 \\
\hline AMD10/1 & 3/3/1999 & $7.82 \mathrm{E}-17$ & $3.78 \mathrm{E}-15$ & $1.87 \mathrm{E}-14$ & $5.13 \mathrm{E}-15$ & 0.7596 & 9.4636 & 0.3843 \\
\hline AMD10/1 & $3 / 29 / 1999$ & $8.97 \mathrm{E}-17$ & $3.12 \mathrm{E}-15$ & $1.43 \mathrm{E}-14$ & $1.38 \mathrm{E}-15$ & 0.2039 & 7.2759 & 0.4403 \\
\hline AMD10/1 & 4/12/1999 & $3.21 \mathrm{E}-17$ & $2.30 \mathrm{E}-15$ & $1.11 \mathrm{E}-14$ & $9.22 \mathrm{E}-16$ & 0.1364 & 5.6433 & 0.1575 \\
\hline AMD10/1 & $5 / 3 / 1999$ & $2.97 \mathrm{E}-17$ & $1.74 \mathrm{E}-15$ & $6.11 \mathrm{E}-15$ & $1.68 \mathrm{E}-17$ & 0.0025 & 3.1003 & 0.1460 \\
\hline AMD10/2 & $3 / 3 / 1998$ & $4.61 \mathrm{E}-17$ & $2.18 \mathrm{E}-16$ & $5.53 \mathrm{E}-16$ & $0.00 \mathrm{E}+00$ & 0.0000 & 0.2805 & 0.2263 \\
\hline AMD10/2 & $10 / 28 / 1998$ & $3.18 \mathrm{E}-17$ & $1.92 \mathrm{E}-16$ & $0.00 \mathrm{E}+00$ & $0.00 \mathrm{E}+00$ & 0.0000 & 0.0000 & 0.1561 \\
\hline AMD10/2 & $11 / 9 / 1998$ & $6.99 \mathrm{E}-18$ & $1.55 \mathrm{E}-16$ & $0.00 \mathrm{E}+00$ & $0.00 \mathrm{E}+00$ & 0.0000 & 0.0000 & 0.0343 \\
\hline AMD10/2 & $12 / 8 / 1998$ & $3.70 \mathrm{E}-18$ & $0.00 \mathrm{E}+00$ & $0.00 \mathrm{E}+00$ & $7.09 \mathrm{E}-17$ & 0.0105 & 0.0000 & 0.0182 \\
\hline AMD10/2 & 1/5/1999 & $0.00 \mathrm{E}+00$ & $3.22 \mathrm{E}-16$ & $0.00 \mathrm{E}+00$ & $1.06 \mathrm{E}-16$ & 0.0156 & 0.0000 & 0.0000 \\
\hline AMD10/2 & 2/1/1999 & $1.54 \mathrm{E}-18$ & $1.37 \mathrm{E}-16$ & $0.00 \mathrm{E}+00$ & $0.00 \mathrm{E}+00$ & 0.0000 & 0.0000 & 0.0076 \\
\hline AMD10/2 & $2 / 16 / 1999$ & $3.01 \mathrm{E}-17$ & 7.69E-17 & $1.91 \mathrm{E}-15$ & $0.00 \mathrm{E}+00$ & 0.0000 & 0.9665 & 0.1478 \\
\hline AMD10/2 & 3/3/1999 & $1.27 \mathrm{E}-17$ & $5.48 \mathrm{E}-17$ & $0.00 \mathrm{E}+00$ & $3.55 \mathrm{E}-17$ & 0.0052 & 0.0000 & 0.0625 \\
\hline AMD10/2 & $3 / 29 / 1999$ & $1.85 \mathrm{E}-17$ & $2.77 \mathrm{E}-16$ & $2.77 \mathrm{E}-16$ & $0.00 \mathrm{E}+00$ & 0.0000 & 0.1403 & 0.0910 \\
\hline AMD10/2 & $5 / 3 / 1999$ & $4.76 \mathrm{E}-17$ & $1.53 \mathrm{E}-15$ & $7.30 \mathrm{E}-15$ & $0.00 \mathrm{E}+00$ & 0.0000 & 3.7030 & 0.2335 \\
\hline AMD10/2 & $7 / 12 / 1999$ & $4.56 \mathrm{E}-17$ & $5.61 \mathrm{E}-15$ & $2.88 \mathrm{E}-14$ & $2.02 \mathrm{E}-15$ & 0.2987 & 14.5880 & 0.2240 \\
\hline AMD10/2 & 8/9/1999 & $4.78 \mathrm{E}-17$ & $3.56 \mathrm{E}-15$ & $1.83 \mathrm{E}-14$ & $1.67 \mathrm{E}-15$ & 0.2478 & 9.2769 & 0.2350 \\
\hline AMD10/2 & 10/11/1999 & $4.55 \mathrm{E}-17$ & $2.83 \mathrm{E}-15$ & $1.25 \mathrm{E}-14$ & $0.00 \mathrm{E}+00$ & 0.0000 & 6.3284 & 0.2234 \\
\hline AMD10/2 & $11 / 8 / 1999$ & $6.59 \mathrm{E}-17$ & $1.70 \mathrm{E}-15$ & $9.48 \mathrm{E}-15$ & $1.86 \mathrm{E}-15$ & 0.2748 & 4.8093 & 0.3236 \\
\hline AMD10/2 & $2 / 7 / 2000$ & $5.54 \mathrm{E}-17$ & $2.86 \mathrm{E}-16$ & $2.66 \mathrm{E}-16$ & $1.13 \mathrm{E}-15$ & 0.1668 & 0.1350 & 0.2723 \\
\hline AMD10/2 & $4 / 10 / 2000$ & $1.21 \mathrm{E}-16$ & $4.78 \mathrm{E}-17$ & $1.67 \mathrm{E}-15$ & $0.00 \mathrm{E}+00$ & 0.0000 & 0.8490 & 0.5951 \\
\hline AMD10/2 & $10 / 16 / 2000$ & $0.00 \mathrm{E}+00$ & $0.00 \mathrm{E}+00$ & $7.59 \mathrm{E}-16$ & $1.98 \mathrm{E}-16$ & 0.0292 & 0.3849 & 0.0000 \\
\hline AMD10/3 & $3 / 3 / 1998$ & $2.77 \mathrm{E}-16$ & $2.43 \mathrm{E}-16$ & $9.11 \mathrm{E}-16$ & $0.00 \mathrm{E}+00$ & 0.0000 & 0.4620 & 1.3617 \\
\hline AMD10/3 & $10 / 28 / 1998$ & $0.00 \mathrm{E}+00$ & $6.53 \mathrm{E}-17$ & $0.00 \mathrm{E}+00$ & $0.00 \mathrm{E}+00$ & 0.0000 & 0.0000 & 0.0000 \\
\hline AMD10/3 & $12 / 21 / 1998$ & $8.90 \mathrm{E}-18$ & $1.49 \mathrm{E}-16$ & $0.00 \mathrm{E}+00$ & $5.36 \mathrm{E}-17$ & 0.0079 & 0.0000 & 0.0437 \\
\hline AMD10/3 & $1 / 10 / 1999$ & $0.00 \mathrm{E}+00$ & $2.64 \mathrm{E}-17$ & $5.12 \mathrm{E}-16$ & $1.11 \mathrm{E}-15$ & 0.1639 & 0.2594 & 0.0000 \\
\hline AMD10/3 & $2 / 16 / 1999$ & $6.09 \mathrm{E}-18$ & $1.47 \mathrm{E}-17$ & $1.81 \mathrm{E}-15$ & $0.00 \mathrm{E}+00$ & 0.0000 & 0.9185 & 0.0299 \\
\hline AMD10/3 & $3 / 16 / 1999$ & $4.98 \mathrm{E}-18$ & $3.42 \mathrm{E}-17$ & $0.00 \mathrm{E}+00$ & $0.00 \mathrm{E}+00$ & 0.0000 & 0.0000 & 0.0244 \\
\hline AMD10/3 & $5 / 3 / 1999$ & $3.15 \mathrm{E}-17$ & $3.75 \mathrm{E}-18$ & $0.00 \mathrm{E}+00$ & $0.00 \mathrm{E}+00$ & 0.0000 & 0.0000 & 0.1546 \\
\hline AMD10/3 & $7 / 12 / 1999$ & $4.31 \mathrm{E}-17$ & $1.29 \mathrm{E}-15$ & $6.69 \mathrm{E}-15$ & $1.03 \mathrm{E}-14$ & 1.5299 & 3.3942 & 0.2117 \\
\hline AMD10/3 & 8/9/1999 & $3.29 \mathrm{E}-17$ & $2.17 \mathrm{E}-15$ & $1.11 \mathrm{E}-14$ & $1.99 \mathrm{E}-15$ & 0.2939 & 5.6496 & 0.1614 \\
\hline AMD10/3 & $9 / 13 / 1999$ & $7.60 \mathrm{E}-17$ & $2.62 \mathrm{E}-15$ & $1.33 \mathrm{E}-14$ & $7.76 \mathrm{E}-16$ & 0.1148 & 6.7359 & 0.3733 \\
\hline AMD10/3 & $11 / 8 / 1999$ & $3.97 \mathrm{E}-17$ & $3.69 \mathrm{E}-15$ & $1.94 \mathrm{E}-14$ & $2.52 \mathrm{E}-15$ & 0.3733 & 9.8581 & 0.1950 \\
\hline AMD10/3 & $2 / 7 / 2000$ & $0.00 \mathrm{E}+00$ & $3.46 \mathrm{E}-15$ & $1.73 \mathrm{E}-14$ & $5.75 \mathrm{E}-17$ & 0.0085 & 8.7707 & 0.0000 \\
\hline AMD10/3 & $4 / 10 / 2000$ & $8.56 \mathrm{E}-17$ & $1.52 \mathrm{E}-15$ & $7.51 \mathrm{E}-15$ & $1.14 \mathrm{E}-15$ & 0.1681 & 3.8062 & 0.4205 \\
\hline
\end{tabular}


Table 4. Xenon Tracer Results from 1996 and 1998 Recharge Experiments.

\begin{tabular}{|c|c|c|c|c|c|c|c|c|}
\hline Sample & $\begin{array}{l}\text { Sample } \\
\text { Date }\end{array}$ & $\begin{array}{l}{ }^{124} \mathrm{Xe} \\
\mathrm{mol} / \mathrm{mol} \mathrm{H} \mathrm{H}_{2} \mathrm{O}\end{array}$ & $\begin{array}{l}{ }^{8} \mathrm{Xe} \\
10 \mathrm{l} / \mathrm{mol} \mathrm{H} \mathrm{H}_{2} \mathrm{O}\end{array}$ & & & $\begin{array}{l}\mathrm{C} / \mathrm{Co}{ }^{136} \mathrm{Xe} \\
\text { as percent }\end{array}$ & $\begin{array}{l}\mathrm{C} / \mathrm{Co}{ }^{129} \mathrm{Xe} \\
\text { as percent }\end{array}$ & $\begin{array}{l}\mathrm{C} / \mathrm{Co}^{124} \mathrm{Xe} \\
\text { as percent }\end{array}$ \\
\hline AMD10/3 & $6 / 12 / 2000$ & $3.97 \mathrm{E}-17$ & $4.88 \mathrm{E}-16$ & $2.38 \mathrm{E}-15$ & $1.51 \mathrm{E}-15$ & 0.2239 & 1.2078 & 0.1949 \\
\hline AMD10/3 & $10 / 16 / 2000$ & $0.00 \mathrm{E}+00$ & $2.09 \mathrm{E}-16$ & $0.00 \mathrm{E}+00$ & $5.34 \mathrm{E}-16$ & 0.0790 & 0.0000 & 0.0000 \\
\hline AMD10/4 & 3/3/1998 & $5.50 \mathrm{E}-16$ & $4.49 \mathrm{E}-16$ & $1.36 \mathrm{E}-15$ & $0.00 \mathrm{E}+00$ & 0.0000 & 0.6874 & 2.6989 \\
\hline AMD10/4 & $10 / 28 / 1998$ & $6.17 \mathrm{E}-16$ & $1.28 \mathrm{E}-16$ & $3.33 \mathrm{E}-16$ & $0.00 \mathrm{E}+00$ & 0.0000 & 0.1690 & 3.0290 \\
\hline AMD10/4 & $12 / 21 / 1998$ & $5.00 \mathrm{E}-16$ & $2.45 \mathrm{E}-16$ & $1.70 \mathrm{E}-16$ & $0.00 \mathrm{E}+00$ & 0.0000 & 0.0863 & 2.4556 \\
\hline AMD10/4 & $1 / 18 / 1999$ & $4.63 \mathrm{E}-16$ & $1.43 \mathrm{E}-16$ & $0.00 \mathrm{E}+00$ & $0.00 \mathrm{E}+00$ & 0.0000 & 0.0000 & 2.2717 \\
\hline AMD10/4 & 2/16/1999 & $3.78 \mathrm{E}-16$ & $4.13 \mathrm{E}-16$ & $1.41 \mathrm{E}-15$ & $3.84 \mathrm{E}-16$ & 0.0569 & 0.7163 & 1.8562 \\
\hline AMD10/4 & 6/9/1999 & $2.08 \mathrm{E}-16$ & $0.00 \mathrm{E}+00$ & $6.05 \mathrm{E}-16$ & $7.60 \mathrm{E}-16$ & 0.1125 & 0.3069 & 1.0235 \\
\hline AMD10/4 & $9 / 13 / 1999$ & $1.07 \mathrm{E}-16$ & $4.32 \mathrm{E}-17$ & $1.58 \mathrm{E}-15$ & $1.14 \mathrm{E}-15$ & 0.1686 & 0.8002 & 0.5248 \\
\hline AMD10/4 & $2 / 7 / 2000$ & $4.50 \mathrm{E}-17$ & $4.85 \mathrm{E}-16$ & $1.26 \mathrm{E}-15$ & $0.00 \mathrm{E}+00$ & 0.0000 & 0.6402 & 0.2210 \\
\hline AMD10/4 & $4 / 10 / 2000$ & $1.00 \mathrm{E}-17$ & $1.76 \mathrm{E}-15$ & $9.00 \mathrm{E}-15$ & $1.27 \mathrm{E}-15$ & 0.1877 & 4.5614 & 0.0491 \\
\hline AMD10/4 & $6 / 12 / 2000$ & $5.46 \mathrm{E}-18$ & $2.24 \mathrm{E}-15$ & $1.10 \mathrm{E}-14$ & $1.90 \mathrm{E}-15$ & 0.2810 & 5.6002 & 0.0268 \\
\hline AMD10/4 & $7 / 17 / 2000$ & $8.72 \mathrm{E}-18$ & $1.90 \mathrm{E}-15$ & $1.16 \mathrm{E}-14$ & $6.75 \mathrm{E}-16$ & 0.0998 & 5.8955 & 0.0428 \\
\hline AMD10/4 & $10 / 16 / 2000$ & $5.81 \mathrm{E}-17$ & $1.46 \mathrm{E}-15$ & $5.35 \mathrm{E}-15$ & 7.89E-17 & 0.0117 & 2.7142 & 0.2853 \\
\hline AMD10/5 & $12 / 21 / 1998$ & $8.62 \mathrm{E}-19$ & $4.73 \mathrm{E}-16$ & $1.47 \mathrm{E}-15$ & $0.00 \mathrm{E}+00$ & 0.0000 & 0.7451 & 0.0042 \\
\hline AMD10/5 & 3/3/1999 & $1.61 \mathrm{E}-17$ & $1.40 \mathrm{E}-16$ & $6.30 \mathrm{E}-17$ & $1.53 \mathrm{E}-16$ & 0.0226 & 0.0320 & 0.0791 \\
\hline AMD10/5 & $10 / 16 / 2000$ & $5.15 \mathrm{E}-17$ & $0.00 \mathrm{E}+00$ & $4.34 \mathrm{E}-15$ & $0.00 \mathrm{E}+00$ & 0.0000 & 2.2029 & 0.2531 \\
\hline AMD11/1 & $10 / 29 / 1998$ & $2.84 \mathrm{E}-17$ & $2.09 \mathrm{E}-16$ & $0.00 \mathrm{E}+00$ & $0.00 \mathrm{E}+00$ & 0.0000 & 0.0000 & 0.1394 \\
\hline AMD11/1 & $1 / 5 / 1999$ & $2.24 \mathrm{E}-17$ & $3.50 \mathrm{E}-16$ & $5.91 \mathrm{E}-16$ & $1.31 \mathrm{E}-17$ & 0.0019 & 0.2997 & 0.1101 \\
\hline AMD11/1 & 2/1/1999 & $1.59 \mathrm{E}-17$ & $1.70 \mathrm{E}-16$ & $0.00 \mathrm{E}+00$ & $0.00 \mathrm{E}+00$ & 0.0000 & 0.0000 & 0.0780 \\
\hline AMD11/1 & $2 / 17 / 1999$ & $1.63 \mathrm{E}-17$ & $0.00 \mathrm{E}+00$ & $4.93 \mathrm{E}-16$ & $0.00 \mathrm{E}+00$ & 0.0000 & 0.2498 & 0.0803 \\
\hline AMD11/1 & 3/4/1999 & $3.02 \mathrm{E}-18$ & $2.07 \mathrm{E}-16$ & $0.00 \mathrm{E}+00$ & $1.32 \mathrm{E}-16$ & 0.0195 & 0.0000 & 0.0148 \\
\hline AMD11/1 & $3 / 15 / 1999$ & $1.01 \mathrm{E}-17$ & $8.43 \mathrm{E}-17$ & $0.00 \mathrm{E}+00$ & $1.41 \mathrm{E}-17$ & 0.0021 & 0.0000 & 0.0498 \\
\hline AMD11/1 & 5/4/1999 & 4.23E-17 & $3.77 \mathrm{E}-16$ & $1.41 \mathrm{E}-16$ & $0.00 \mathrm{E}+00$ & 0.0000 & 0.0714 & 0.2077 \\
\hline AMD11/2 & $3 / 4 / 1998$ & E-17 & E-16 & E-15 & $0.00 \mathrm{E}+00$ & 0.0000 & 0.6644 & 0.1933 \\
\hline AMD $11 / 2$ & $10 / 29 / 1998$ & $6.96 \mathrm{E}-17$ & $3.54 \mathrm{E}-16$ & $2.73 \mathrm{E}-16$ & $9.36 \mathrm{E}-17$ & 0.0139 & 0.1384 & 0.3417 \\
\hline AMD11/2 & $11 / 9 / 1998$ & $6.35 \mathrm{E}-17$ & $2.04 \mathrm{E}-16$ & $3.32 \mathrm{E}-16$ & $9.92 \mathrm{E}-17$ & 0.0147 & 0.1685 & 0.3118 \\
\hline AMD11/2 & $12 / 21 / 1998$ & $1.16 \mathrm{E}-16$ & $2.76 \mathrm{E}-16$ & $0.00 \mathrm{E}+00$ & $0.00 \mathrm{E}+00$ & 0.0000 & 0.0000 & 0.5694 \\
\hline AMD11/2 & $1 / 5 / 1999$ & $1.06 \mathrm{E}-16$ & $3.57 \mathrm{E}-16$ & $5.26 \mathrm{E}-17$ & $0.00 \mathrm{E}+00$ & 0.0000 & 0.0267 & 0.5229 \\
\hline AMD $11 / 2$ & 2/1/1999 & $1.35 \mathrm{E}-16$ & $3.49 \mathrm{E}-16$ & $6.27 \mathrm{E}-16$ & $0.00 \mathrm{E}+00$ & 0.0000 & 0.3177 & 0.6605 \\
\hline AMD11/2 & 2/17/1999 & $3.85 \mathrm{E}-17$ & $1.15 \mathrm{E}-16$ & $1.47 \mathrm{E}-15$ & $0.00 \mathrm{E}+00$ & 0.0000 & 0.7474 & 0.1890 \\
\hline AMD11/2 & 3/4/1999 & $1.49 \mathrm{E}-16$ & $2.62 \mathrm{E}-16$ & $4.95 \mathrm{E}-16$ & $8.16 \mathrm{E}-18$ & 0.0012 & 0.2509 & 0.7312 \\
\hline AMD $11 / 2$ & 4/12/1999 & $2.08 \mathrm{E}-16$ & $4.44 \mathrm{E}-16$ & $6.15 \mathrm{E}-16$ & $0.00 \mathrm{E}+00$ & 0.0000 & 0.3119 & 1.0229 \\
\hline AMD $11 / 2$ & $5 / 4 / 1999$ & $2.50 \mathrm{E}-16$ & $9.42 \mathrm{E}-16$ & $1.68 \mathrm{E}-15$ & $0.00 \mathrm{E}+00$ & 0.0000 & 0.8508 & 1.2270 \\
\hline AMD11/2 & $7 / 24 / 2000$ & $1.24 \mathrm{E}-16$ & $2.50 \mathrm{E}-17$ & $0.00 \mathrm{E}+00$ & $1.12 \mathrm{E}-15$ & 0.1660 & 0.0000 & 0.6106 \\
\hline AMD11/2 & $10 / 23 / 2000$ & $3.74 \mathrm{E}-17$ & $3.28 \mathrm{E}-16$ & $8.33 \mathrm{E}-15$ & $2.42 \mathrm{E}-15$ & 0.3583 & 4.2228 & 0.1836 \\
\hline AMD11/3 & $10 / 29 / 1998$ & $3.33 \mathrm{E}-17$ & $2.69 \mathrm{E}-16$ & $0.00 \mathrm{E}+00$ & $0.00 \mathrm{E}+00$ & 0.0000 & 0.0000 & 0.1635 \\
\hline AMD11/3 & $12 / 21 / 1998$ & $1.47 \mathrm{E}-17$ & $1.61 \mathrm{E}-16$ & $0.00 \mathrm{E}+00$ & $0.00 \mathrm{E}+00$ & 0.0000 & 0.0000 & 0.0720 \\
\hline AMD11/3 & 1/18/1999 & $3.97 \mathrm{E}-17$ & $0.00 \mathrm{E}+00$ & $5.07 \mathrm{E}-16$ & $0.00 \mathrm{E}+00$ & 0.0000 & 0.2569 & 0.1949 \\
\hline AMD11/3 & 2/17/1999 & $3.10 \mathrm{E}-17$ & $6.81 \mathrm{E}-17$ & $2.52 \mathrm{E}-15$ & $0.00 \mathrm{E}+00$ & 0.0000 & 1.2761 & 0.1521 \\
\hline AMD11/3 & $5 / 4 / 1999$ & $2.89 \mathrm{E}-17$ & $2.19 \mathrm{E}-16$ & $0.00 \mathrm{E}+00$ & $0.00 \mathrm{E}+00$ & 0.0000 & 0.0000 & 0.1420 \\
\hline AMD11/3 & $7 / 24 / 2000$ & $3.44 \mathrm{E}-17$ & $0.00 \mathrm{E}+00$ & $0.00 \mathrm{E}+00$ & $5.05 \mathrm{E}-16$ & 0.0748 & 0.0000 & 0.1691 \\
\hline AMD11/3 & $10 / 23 / 2000$ & $0.00 \mathrm{E}+00$ & $9.80 \mathrm{E}-18$ & $0.00 \mathrm{E}+00$ & $1.44 \mathrm{E}-15$ & 0.2138 & 0.0000 & 0.0000 \\
\hline AMD11/4 & 3/4/1998 & $2.93 \mathrm{E}-18$ & $3.13 \mathrm{E}-16$ & $1.07 \mathrm{E}-15$ & $0.00 \mathrm{E}+00$ & 0.0000 & 0.5419 & 0.0144 \\
\hline AMD11/4 & $10 / 29 / 1998$ & $0.00 \mathrm{E}+00$ & $2.04 \mathrm{E}-16$ & $3.51 \mathrm{E}-16$ & $1.12 \mathrm{E}-16$ & 0.0165 & 0.1778 & 0.0000 \\
\hline AMD11/4 & $12 / 21 / 1998$ & $4.70 \mathrm{E}-18$ & $2.30 \mathrm{E}-16$ & $0.00 \mathrm{E}+00$ & $0.00 \mathrm{E}+00$ & 0.0000 & 0.0000 & 0.0231 \\
\hline
\end{tabular}


Table 4. Xenon Tracer Results from 1996 and 1998 Recharge Experiments.

\begin{tabular}{|c|c|c|c|c|c|c|c|c|}
\hline Sample & $\begin{array}{l}\text { Sample } \\
\text { Date }\end{array}$ & $\begin{array}{l}{ }^{124} \mathrm{Xe} \\
\mathrm{mol} / \mathrm{mol} \mathrm{H} \mathrm{H}_{2} \mathrm{O}\end{array}$ & & & 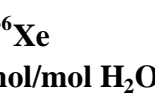 & $\begin{array}{c}\mathrm{C} / \mathrm{Co}{ }^{136} \mathrm{Xe} \\
\text { as percent }\end{array}$ & $\begin{array}{l}\mathrm{C} / \mathrm{Co}{ }^{129} \mathrm{Xe} \\
\text { as percent }\end{array}$ & $\begin{array}{l}\mathrm{C} / \mathrm{Co}^{124} \mathrm{Xe} \\
\text { as percent }\end{array}$ \\
\hline AMD11/4 & $1 / 18 / 1999$ & $8.10 \mathrm{E}-19$ & $0.00 \mathrm{E}+00$ & $1.22 \mathrm{E}-16$ & $0.00 \mathrm{E}+00$ & 0.0000 & 0.0619 & 0.0040 \\
\hline AMD11/4 & 2/17/1999 & $1.80 \mathrm{E}-17$ & $3.74 \mathrm{E}-17$ & $2.14 \mathrm{E}-17$ & $0.00 \mathrm{E}+00$ & 0.0000 & 0.0108 & 0.0882 \\
\hline AMD11/4 & $5 / 4 / 1999$ & $5.91 \mathrm{E}-17$ & $2.03 \mathrm{E}-16$ & $2.32 \mathrm{E}-15$ & $0.00 \mathrm{E}+00$ & 0.0000 & 1.1743 & 0.2901 \\
\hline AMD11/4 & $7 / 24 / 2000$ & $0.00 \mathrm{E}+00$ & $0.00 \mathrm{E}+00$ & $1.42 \mathrm{E}-15$ & $2.51 \mathrm{E}-16$ & 0.0371 & 0.7218 & 0.0000 \\
\hline AMD11/4 & $10 / 23 / 2000$ & $0.00 \mathrm{E}+00$ & $0.00 \mathrm{E}+00$ & $5.57 \mathrm{E}-16$ & $0.00 \mathrm{E}+00$ & 0.0000 & 0.2826 & 0.0000 \\
\hline AMD11/5 & $3 / 4 / 1999$ & $1.85 \mathrm{E}-18$ & $1.60 \mathrm{E}-16$ & $0.00 \mathrm{E}+00$ & $1.39 \mathrm{E}-16$ & 0.0206 & 0.0000 & 0.0091 \\
\hline AMD11/5 & $7 / 24 / 2000$ & $3.93 \mathrm{E}-17$ & $1.74 \mathrm{E}-16$ & $3.97 \mathrm{E}-15$ & $9.05 \mathrm{E}-16$ & 0.1339 & 2.0154 & 0.1931 \\
\hline AMD11/5 & $10 / 23 / 2000$ & $0.00 \mathrm{E}+00$ & $2.23 \mathrm{E}-16$ & $8.00 \mathrm{E}-16$ & $1.20 \mathrm{E}-16$ & 0.0178 & 0.4058 & 0.0000 \\
\hline AMD9/2 & $2 / 18 / 1997$ & $1.91 \mathrm{E}-15$ & $3.01 \mathrm{E}-16$ & $7.53 \mathrm{E}-16$ & $3.97 \mathrm{E}-17$ & 0.0059 & 0.3817 & 9.4031 \\
\hline AMD9/2 & $3 / 31 / 1997$ & $4.09 \mathrm{E}-15$ & $5.72 \mathrm{E}-16$ & $1.69 \mathrm{E}-15$ & $1.73 \mathrm{E}-16$ & 0.0257 & 0.8577 & 20.1040 \\
\hline AMD9/3 & $1 / 30 / 1997$ & $4.32 \mathrm{E}-17$ & $0.00 \mathrm{E}+00$ & $0.00 \mathrm{E}+00$ & $1.12 \mathrm{E}-16$ & 0.0165 & 0.0000 & 0.2120 \\
\hline AMD9/3 & $3 / 31 / 1997$ & $0.00 \mathrm{E}+00$ & $0.00 \mathrm{E}+00$ & $0.00 \mathrm{E}+00$ & $4.35 \mathrm{E}-16$ & 0.0644 & 0.0000 & 0.0000 \\
\hline Ana St2B & $10 / 11 / 1996$ & $2.05 \mathrm{E}-14$ & $1.32 \mathrm{E}-15$ & $1.30 \mathrm{E}-14$ & $8.00 \mathrm{E}-17$ & 0.0118 & 6.6139 & 100.6000 \\
\hline Ana St2M & $10 / 11 / 1996$ & $2.02 \mathrm{E}-14$ & $1.38 \mathrm{E}-15$ & $1.24 \mathrm{E}-14$ & $1.15 \mathrm{E}-16$ & 0.0170 & 6.2946 & 99.2810 \\
\hline Ana St3B & $10 / 11 / 1996$ & $2.10 \mathrm{E}-14$ & $1.29 \mathrm{E}-15$ & $1.29 \mathrm{E}-14$ & $0.00 \mathrm{E}+00$ & 0.0000 & 6.5169 & 103.2300 \\
\hline Ana St4M & $10 / 11 / 1996$ & $1.97 \mathrm{E}-14$ & $1.22 \mathrm{E}-15$ & $1.24 \mathrm{E}-14$ & $3.84 \mathrm{E}-17$ & 0.0057 & 6.2625 & 96.8820 \\
\hline DINKA & $10 / 16 / 2000$ & $0.00 \mathrm{E}+00$ & $0.00 \mathrm{E}+00$ & $0.00 \mathrm{E}+00$ & $2.42 \mathrm{E}-15$ & 0.3575 & 0.0000 & 0.0000 \\
\hline FKIM2 & $10 / 18 / 2000$ & $1.48 \mathrm{E}-17$ & $3.49 \mathrm{E}-16$ & $9.57 \mathrm{E}-15$ & $5.10 \mathrm{E}-15$ & 0.7551 & 4.8524 & 0.0726 \\
\hline FM2 & $10 / 25 / 2000$ & $0.00 \mathrm{E}+00$ & $1.33 \mathrm{E}-17$ & $5.52 \mathrm{E}-15$ & $1.56 \mathrm{E}-15$ & 0.2308 & 2.7988 & 0.0000 \\
\hline K Basin & $10 / 7 / 1998$ & $2.79 \mathrm{E}-17$ & $1.11 \mathrm{E}-16$ & $1.10 \mathrm{E}-16$ & $6.79 \mathrm{E}-13$ & 100.5400 & 0.0557 & 0.1372 \\
\hline K Basin & $10 / 7 / 1998$ & $0.00 \mathrm{E}+00$ & $0.00 \mathrm{E}+00$ & $0.00 \mathrm{E}+00$ & $7.15 \mathrm{E}-13$ & 105.7700 & 0.0000 & 0.0000 \\
\hline K Basin & $10 / 7 / 1998$ & $1.26 \mathrm{E}-17$ & $0.00 \mathrm{E}+00$ & $0.00 \mathrm{E}+00$ & $6.69 \mathrm{E}-13$ & 99.0440 & 0.0000 & 0.0618 \\
\hline K Basin & $10 / 7 / 1998$ & $3.54 \mathrm{E}-17$ & $3.32 \mathrm{E}-17$ & $3.04 \mathrm{E}-16$ & $6.39 \mathrm{E}-13$ & 94.5560 & 0.1542 & 0.1741 \\
\hline K Basin & $10 / 7 / 1998$ & $2.44 \mathrm{E}-17$ & $0.00 \mathrm{E}+00$ & $0.00 \mathrm{E}+00$ & $6.76 \mathrm{E}-13$ & 100.1000 & 0.0000 & 0.1199 \\
\hline KB1 & $10 / 8 / 1998$ & 8.99E-18 & $3.06 \mathrm{E}-17$ & $1.41 \mathrm{E}-16$ & $1.51 \mathrm{E}-16$ & 0.0224 & 0.0717 & 0.0441 \\
\hline KB1 & $10 / 15 / 1998$ & $0.00 \mathrm{E}+00$ & $0.00 \mathrm{E}+00$ & $0.00 \mathrm{E}+00$ & $6.62 \mathrm{E}-16$ & 0.0980 & 0.0000 & 0.0000 \\
\hline KB1 & $10 / 22 / 1998$ & $2.58 \mathrm{E}-17$ & $0.00 \mathrm{E}+00$ & $5.60 \mathrm{E}-16$ & $1.14 \mathrm{E}-13$ & 16.8130 & 0.2839 & 0.1268 \\
\hline KB1 & $10 / 28 / 1998$ & $0.00 \mathrm{E}+00$ & $1.03 \mathrm{E}-16$ & $1.39 \mathrm{E}-16$ & $9.04 \mathrm{E}-14$ & 13.3750 & 0.0707 & 0.0000 \\
\hline KB1 & $11 / 4 / 1998$ & $2.83 \mathrm{E}-17$ & $0.00 \mathrm{E}+00$ & $0.00 \mathrm{E}+00$ & $2.55 \mathrm{E}-14$ & 3.7684 & 0.0000 & 0.1392 \\
\hline KB1 & $11 / 9 / 1998$ & $0.00 \mathrm{E}+00$ & $6.02 \mathrm{E}-17$ & $6.90 \mathrm{E}-16$ & $2.86 \mathrm{E}-15$ & 0.4235 & 0.3500 & 0.0000 \\
\hline KB1 & $11 / 19 / 1998$ & $0.00 \mathrm{E}+00$ & $0.00 \mathrm{E}+00$ & $0.00 \mathrm{E}+00$ & $3.19 \mathrm{E}-15$ & 0.4721 & 0.0000 & 0.0000 \\
\hline KB1 & $11 / 23 / 1998$ & $2.15 \mathrm{E}-17$ & $1.59 \mathrm{E}-16$ & $1.82 \mathrm{E}-16$ & $1.93 \mathrm{E}-15$ & 0.2852 & 0.0921 & 0.1053 \\
\hline KB1 & $11 / 30 / 1998$ & $1.05 \mathrm{E}-17$ & $9.63 \mathrm{E}-17$ & $5.95 \mathrm{E}-16$ & $3.43 \mathrm{E}-15$ & 0.5081 & 0.3015 & 0.0513 \\
\hline KB1 & $12 / 29 / 1998$ & $1.88 \mathrm{E}-17$ & $3.90 \mathrm{E}-16$ & $0.00 \mathrm{E}+00$ & $1.37 \mathrm{E}-15$ & 0.2023 & 0.0000 & 0.0925 \\
\hline KB1 & $1 / 25 / 1999$ & $0.00 \mathrm{E}+00$ & $2.90 \mathrm{E}-16$ & $0.00 \mathrm{E}+00$ & $6.30 \mathrm{E}-16$ & 0.0932 & 0.0000 & 0.0000 \\
\hline KBS1 & $12 / 30 / 1996$ & $3.14 \mathrm{E}-18$ & $0.00 \mathrm{E}+00$ & $0.00 \mathrm{E}+00$ & $0.00 \mathrm{E}+00$ & 0.0000 & 0.0000 & 0.0154 \\
\hline KBS1 & $1 / 27 / 1997$ & $2.17 \mathrm{E}-17$ & $2.75 \mathrm{E}-16$ & $1.05 \mathrm{E}-16$ & $1.18 \mathrm{E}-16$ & 0.0175 & 0.0533 & 0.1066 \\
\hline KBS1 & $2 / 21 / 1997$ & 8.82E-18 & $5.43 \mathrm{E}-17$ & $5.06 \mathrm{E}-16$ & $0.00 \mathrm{E}+00$ & 0.0000 & 0.2565 & 0.0433 \\
\hline KBS1 & 4/1/1997 & $0.00 \mathrm{E}+00$ & $1.10 \mathrm{E}-16$ & $0.00 \mathrm{E}+00$ & $0.00 \mathrm{E}+00$ & 0.0000 & 0.0000 & 0.0000 \\
\hline KBS1 & $10 / 8 / 1998$ & $1.37 \mathrm{E}-17$ & $8.95 \mathrm{E}-17$ & $8.76 \mathrm{E}-17$ & $0.00 \mathrm{E}+00$ & 0.0000 & 0.0444 & 0.0673 \\
\hline KBS1 & $10 / 16 / 1998$ & $3.62 \mathrm{E}-17$ & $0.00 \mathrm{E}+00$ & $0.00 \mathrm{E}+00$ & $2.57 \mathrm{E}-13$ & 38.0910 & 0.0000 & 0.1778 \\
\hline KBS1 & $10 / 21 / 1998$ & $0.00 \mathrm{E}+00$ & $1.84 \mathrm{E}-16$ & $5.10 \mathrm{E}-17$ & $3.54 \mathrm{E}-14$ & 5.2456 & 0.0259 & 0.0000 \\
\hline KBS1 & $10 / 28 / 1998$ & $2.08 \mathrm{E}-18$ & $8.32 \mathrm{E}-17$ & $0.00 \mathrm{E}+00$ & 7.07E-15 & 1.0463 & 0.0000 & 0.0102 \\
\hline KBS1 & $11 / 9 / 1998$ & $0.00 \mathrm{E}+00$ & $6.08 \mathrm{E}-17$ & $0.00 \mathrm{E}+00$ & $5.19 \mathrm{E}-14$ & 7.6814 & 0.0000 & 0.0000 \\
\hline
\end{tabular}


Table 4. Xenon Tracer Results from 1996 and 1998 Recharge Experiments.

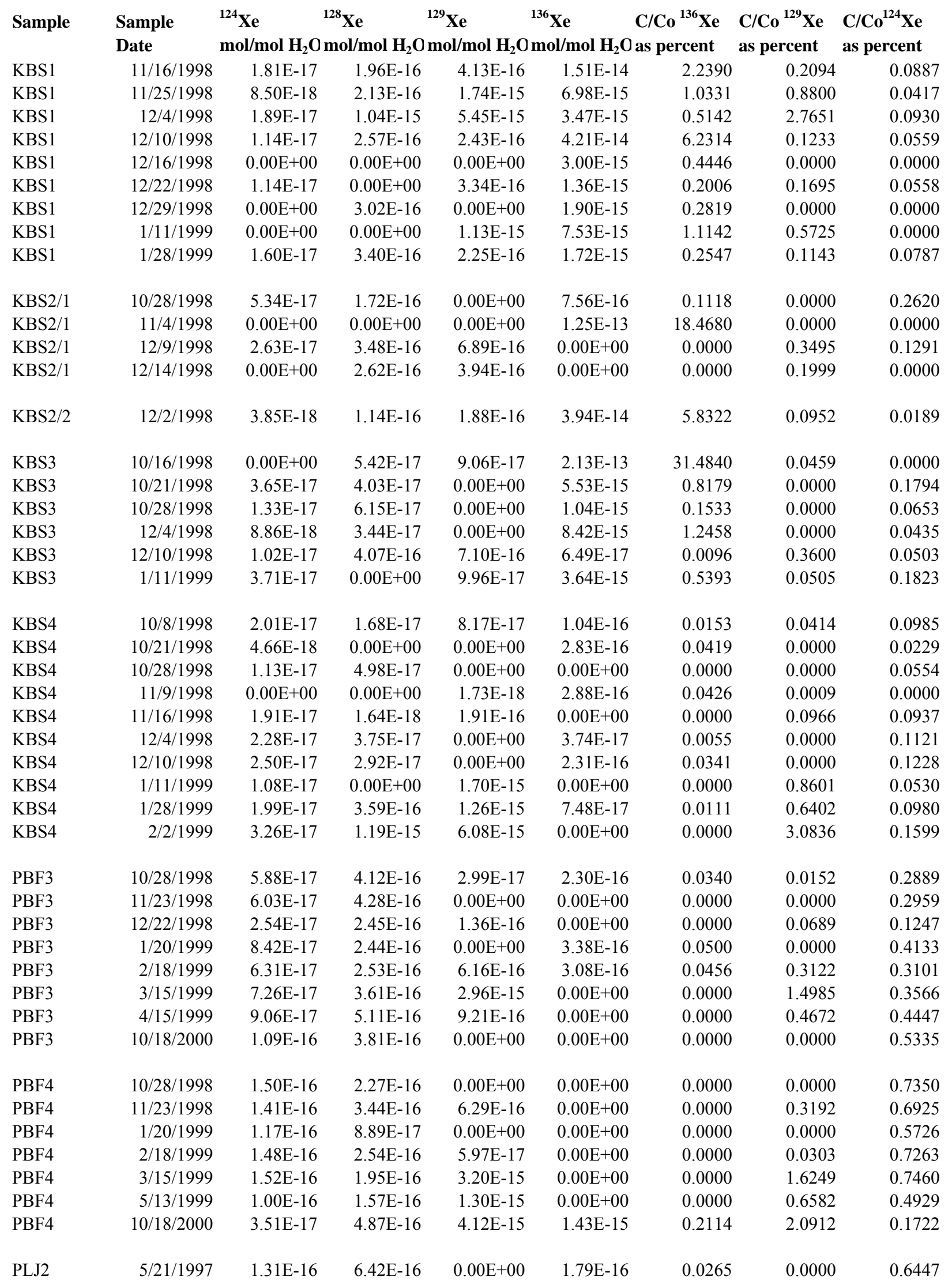


Table 4. Xenon Tracer Results from 1996 and 1998 Recharge Experiments.

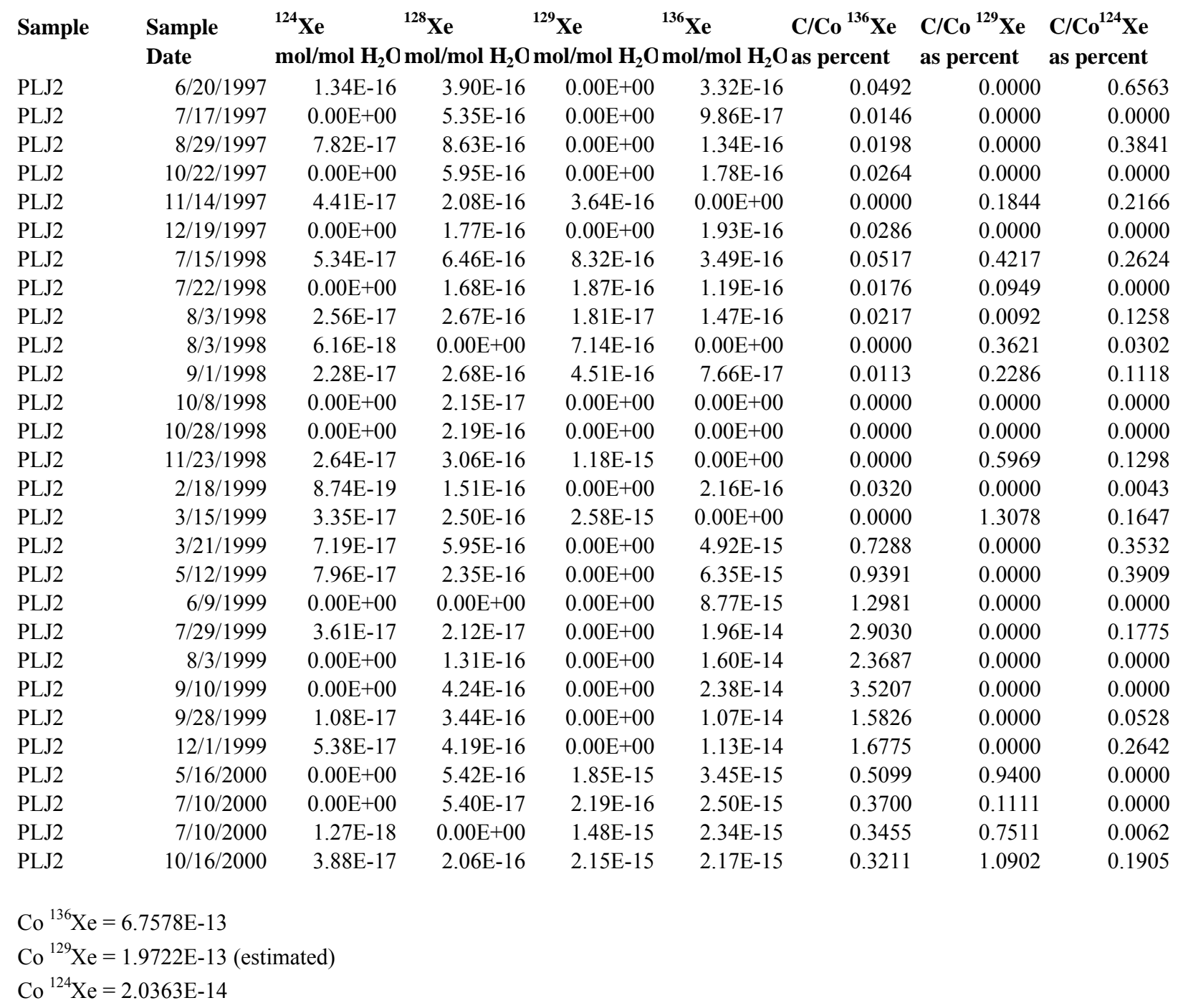


In wells A-28 and ABS-2, the $\delta^{18} \mathrm{O}$ value decreased within 15-30 days after Colorado River started to infiltrate, but at a much slower rate and only reaching a minimum value of -9.5 per mil at approximately 90 days (Fig. 15b). It required over 200 days from when Colorado River water recharge began for the $\delta^{18} \mathrm{O}$ value of groundwater in these wells to return to an SAR value. It is noteworthy that A-28 and ABS-2 are located on opposite sides of Anaheim Lake and are perforated at different depths yet the $\delta^{18} \mathrm{O}$ pattern of Colorado River breakthrough was nearly identical. Interestingly, both these wells had a ${ }^{3} \mathrm{H}-{ }^{3} \mathrm{He}$ age of greater than two years, while the ${ }^{3} \mathrm{H}-{ }^{3} \mathrm{He}$ age of AMD-9 level 1 and A-27 were less than one year.

Groundwater in AMD-9 level 2 also had ${ }^{3} \mathrm{H}-{ }^{3} \mathrm{He}$ age of greater than two years measured in September 1996. At approximately 150 days after Colorado River water infiltration began, the $\delta^{18} \mathrm{O}$ value of AMD-9 level 2 began to decrease and reached a minimum of -9.8 per mil at approximately 180 days (Fig. 15a).

The $\delta^{18} \mathrm{O}$ value of monitoring well AM-44 began to decrease somewhere between 40 and 100 days after Colorado River started infiltration (Fig. 15b). At approximately 140 days, the $\delta^{18} \mathrm{O}$ reached a minimum value of -10.6 per mil, and then returned to an SAR value by approximately 230 days. Monitoring well AM-44 is approximately 1000 feet downgradient from the western edge of Anaheim Lake and is perforated at a shallower depth than AMD-9 level 1 or A-27.

All other groundwater wells monitored during this recharge experiment did not produce $\delta^{18} \mathrm{O}$ values significantly lower than that observed for the SAR, indicating little or no Colorado River water reached these wells in the 300 day monitoring period. It is possible that a small percentage of Colorado River water reached these wells, but that the natural variability in the SAR water source combined with the analytical precision $( \pm 0.1$ per mil) masked any changes in $\delta^{18} \mathrm{O}$.

It is useful to calculate the mixing ratios of Colorado River water with SAR water using the $\delta^{18} \mathrm{O}$ value in wells where breakthrough was easily detected. This can be done simply by recognizing that

$$
F=\frac{\delta_{\text {meas }}-\delta_{\text {SAR }}}{\delta_{C O R}-\delta_{S A R}}
$$

where $F$ is the fraction of Colorado River water mixed in a sample, $\delta_{\text {meas }}$ is the measured $\delta^{18} \mathrm{O}$ value, $\delta_{S A R}$ is the initial $\delta^{18} \mathrm{O}$ value in a particular well before the Colorado River tracer arrival, and $\delta_{C O R}$ is the $\delta^{18} \mathrm{O}$ of the Colorado River water measured in Anaheim Lake. The $\delta_{S A R}$ value for each well is an average of all $\delta^{18} \mathrm{O}$ measurements before any decrease toward a Colorado River signature.

Well AMD-9 level 1 reached 100\% Colorado River water tracer, while well A-27 was approximately 94\% Colorado River water. ABS-2 and A-28 were approximately 50\% Colorado River, while AM-44 reached $89 \%$ of a Colorado River water isotope value. AMD-9 level 2 received about a $60 \%$ mixture of Colorado River water. Note that for wells that had ${ }^{3} \mathrm{H}-{ }^{3} \mathrm{He}$ ages greater than one year old, the maximum percent of Colorado River water tracer reaching these sites was lower than for wells that had ages less than one year. This implies that mixing of groundwater from different aquifer layers occurs in wells adjacent to Anaheim Lake. These different aquifer layers have different 
groundwater ages. It is not entirely clear whether the ${ }^{3} \mathrm{H}_{-}{ }^{3} \mathrm{He}$ ages and the $\delta^{18} \mathrm{O}$ values reflect mixing of these different age groundwaters within aquifers or mixing within the well. Perforation intervals may be producing water from more than one layer.

Older groundwater beneath Anaheim Lake has a $\delta_{S A R}$ isotopic value, which can be inferred to represent recharge from the SAR upgradient of this basin. However, the older groundwater could also represent mixtures of older Anaheim Lake recharge, providing that SAR was the predominant diversion source to the basin over the time period represented by the older water. It is probably more reasonable to suggest that the older groundwater is derived upgradient of Anaheim Lake. This is based on the fact that groundwater elevations increase upgradient of Anaheim Lake and the basin is semiannually drained for cleaning, which periodically eliminates groundwater mounding beneath the basin.

\subsection{3 ${ }^{124} \mathrm{Xe}$ Results}

The addition of Colorado River water into Anaheim Lake transpired over an approximately 50-day period, whereas the ${ }^{124} \mathrm{Xe}$ tracer was added in one day. The total ${ }^{124} \mathrm{Xe}$ mass added to the lake was not well known. However, measurements were made at mid-depth and the bottom of the lake within 24 hours after the tracer introduction. The measurements revealed a well-mixed tracer concentration of $2 \times 10^{-14} \mathrm{mmol} / \mathrm{mmolH}_{2} \mathrm{O}( \pm$ $\left.0.1 \times 10^{-14}\right)$. Even though this value appears to be a reliable tracer input concentration, mass balance problems arise while integrating the ${ }^{124} \mathrm{Xe}$ concentration profiles measured over time in nearby wells (A-28 and AM-44), which typically resulted in an integrated ${ }^{124} \mathrm{Xe}$ mass greater than ten times that represented by the measured concentration in the lake. Therefore, adjustments were made to the initial concentration of the ${ }^{124} \mathrm{Xe}$ tracer in Anaheim Lake to reconcile a large part of the discrepancy with well data. A similar condition is further documented in the 1998 Anaheim Lake tracer study discussed below.

Based on the modified input concentration of ${ }^{124} \mathrm{Xe}$, the tracer duration in Anaheim Lake was modeled over time (Fig.16a). The modeling assumes that on a daily basis the ${ }^{124} \mathrm{Xe}$ was well mixed throughout the Anaheim Lake basin, and that no atmospheric loss occurred. The concentration was calculated on a daily time step by calculating the total mass left in the basin minus that removed from daily percolation. Total storage and percolation rates for the basin are well known based on measurement records collected by the District's Forebay operations. Atmospheric loss from upward molecular diffusion was anticipated to be negligible, since rates in water typically are slow (e.g. $10^{-5} \mathrm{~cm}^{2} / \mathrm{s}$ ). However, atmospheric loss due to water turbulence could have enhance diffusional loss several orders of magnitude. No data is available on the turbulence of the Anaheim Lake basin at the time of the tracer introduction, even though fluid motion had to be occurring in order to have a constant input and percolation to the system. More than likely, though, these flows were laminar and the lake was appreciably stratified thermally in order to maintain a thermocline commonly observed in late summer. Furthermore, since the ${ }^{124} \mathrm{Xe}$ concentration profiles measured in downgradient wells following the tracer introduction could only be reconciled on a mass basis with the total mass introduced into the basin (rather than the measured concentration), it is unlikely that any significant loss of ${ }^{124} \mathrm{Xe}$ occurred. 
The ${ }^{124} \mathrm{Xe}$ concentration resulting from the tracer addition was greater than 100 times above natural abundance, providing a large dynamic range for distinguishing small amounts of recharge water mixed with groundwater sampled in a well.
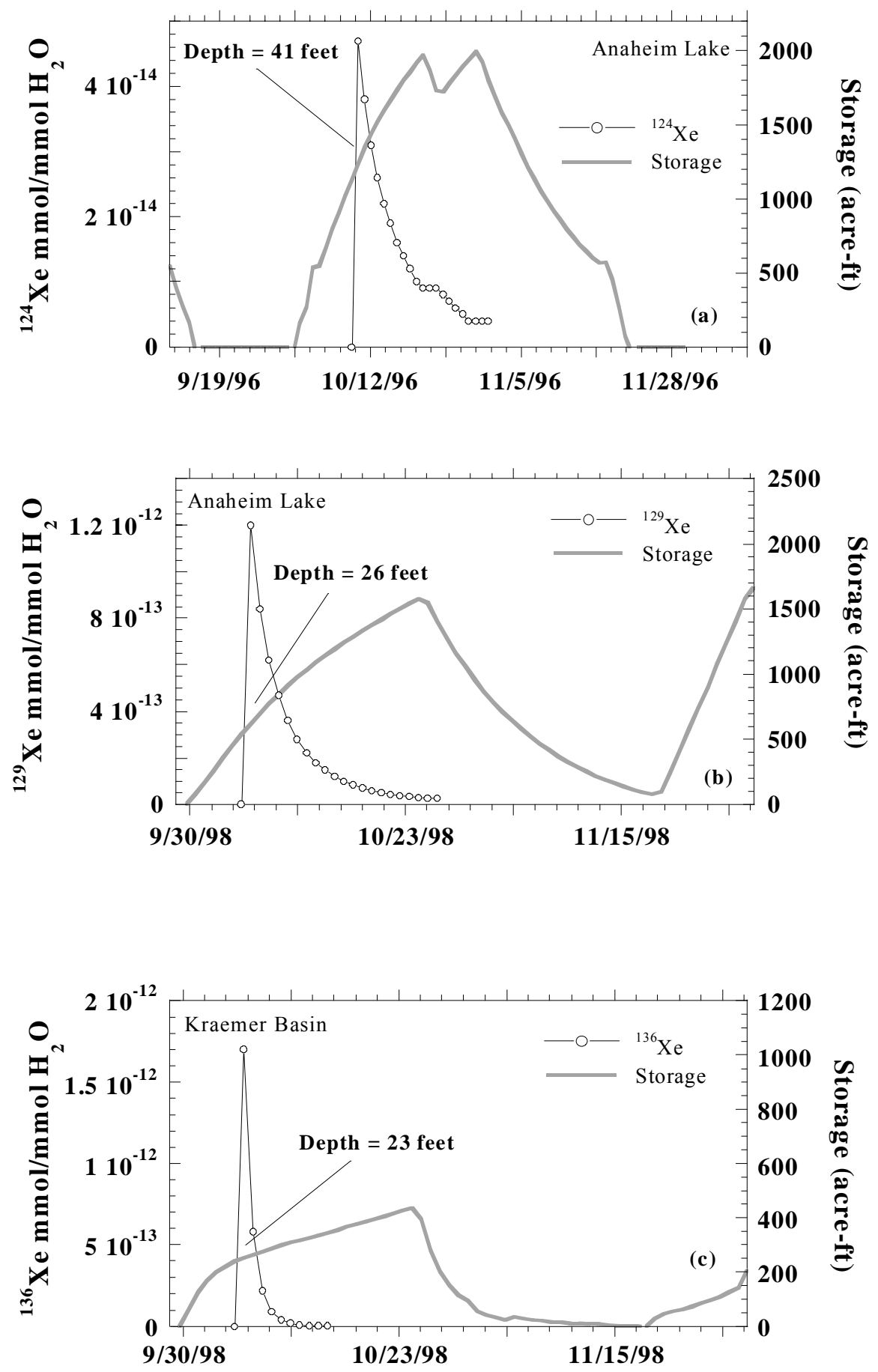

Figure 16. The concentration history of the xenon isotope tracer spike in each basin was modeled for a) Anaheim Lake in 1996, b) Anaheim Lake in 1998, and c) Kraemer Basin in 1998. 
This large dynamic range was best illustrated in the groundwater for deep production well A-44, where ${ }^{124} \mathrm{Xe}$ was detected at approximately 6 months after introduction and persisted until late summer of 1997 (Fig. 17a). Compared to the concentration of ${ }^{124} \mathrm{Xe}$ measured in Anaheim Lake $\left(2 \times 10^{-14} \mathrm{mmol}^{124} \mathrm{Xe} / \mathrm{mmolH}_{2} \mathrm{O}\right)$, the detected amount of ${ }^{124} \mathrm{Xe}$ in A-44 (approximately $5 \times 10^{-16} \mathrm{mmol}^{124} \mathrm{Xe} / \mathrm{mmolH}_{2} \mathrm{O}$ ) was about 2 to $3 \%$ of the lake concentration. Nevertheless, its detection in several sequential samples indicates that a small component of Anaheim Lake recharge reaches this production in less than one year.
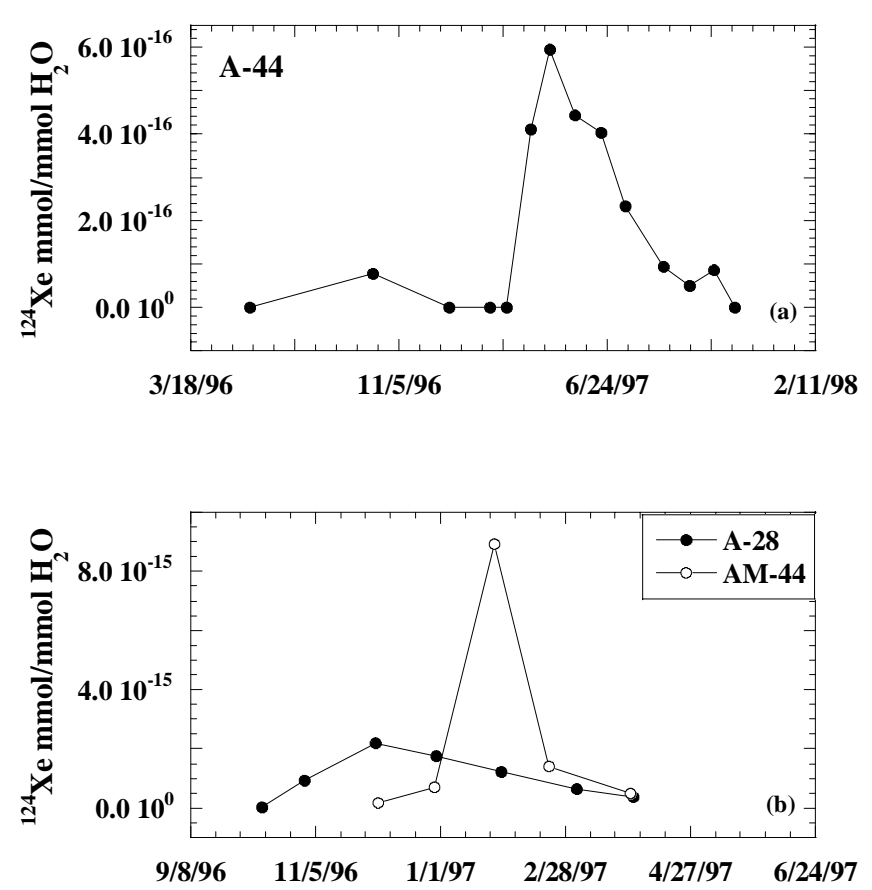

Figure 17. Breakthrough curves of ${ }^{124}$ Xe tracer for a) well A-44, b) wells A-28 and AM44.

A comparison of the ${ }^{124} \mathrm{Xe}$ and the $\delta^{18} \mathrm{O}$ results for wells A-28 and AM-44 illustrate the behavior of the ${ }^{124} \mathrm{Xe}$ as a tracer for these recharge conditions (Fig. 17b). In both of these wells the center of mass for the ${ }^{124} \mathrm{Xe}$ precedes the center of mass for the $\delta^{18} \mathrm{O}$ value. The total duration of the Colorado River water loading in Anaheim Lake was 50 days, and, as a result, the half-width of the recharge pulse travelling through the subsurface is $\sim 25$ days. The ${ }^{124} \mathrm{Xe}$ tracer was introduced in one day, 11 days after the Colorado River was first infiltrated, which is less than one-quarter of the 50 day Colorado River recharge pulse. Therefore, the center of mass for the ${ }^{124} \mathrm{Xe}$ tracer pulse travelling through the subsurface should always precede that of the Colorado River as delineated by the $\delta^{18} \mathrm{O}$ measurements. If the ${ }^{124} \mathrm{Xe}$ was significantly retarded by gas partitioning in the vadose zone, then the center of mass for ${ }^{124} \mathrm{Xe}$ would likely not precede that of the $\delta^{18} \mathrm{O}$.

The apparent conservative transport behavior below Anaheim Lake for the ${ }^{124} \mathrm{Xe}$ tracer is important in regards to recharge mechanisms. In order to introduce the ${ }^{124} \mathrm{Xe}$ tracer into Anaheim Lake, the dissolved xenon concentration had to exceed equilibrium solubility. As a result, any significant encounter with unsaturated zone conditions below 
the lake during recharge should have caused loss of xenon into the gas phase. This loss would have retarded the transport progress of the ${ }^{124} \mathrm{Xe}$ and an obvious lag between Colorado River water transport rates and ${ }^{124} \mathrm{Xe}$ rates might have been observed. On the contrary, the ${ }^{124}$ Xe tracer showed no such lag, and as expected and already discussed above, the center of mass for ${ }^{124} \mathrm{Xe}$ preceded that of the Colorado River water isotopic signature.

\subsection{Anaheim Lake and Kraemer Basin Recharge Experiment}

The results from the 1996 Anaheim Lake artificial recharge experiment illustrated the utility of using both $\delta^{18} \mathrm{O}$ and an isotopically-enriched xenon tracer for delineating groundwater travel times to individual wells. In particular, the $\delta^{18} \mathrm{O}$ of Colorado River water was easily detected in nearby wells when it comprised $>10 \%$ of the recharge source. Since the $\delta^{18} \mathrm{O}$ is measured on the actual water molecule, it makes an ideal conservative tracer of the water. However, for $<10 \%$ contributions from Colorado River water, the $\delta^{18} \mathrm{O}$ loses resolving power because of variability in the SAR isotopic value. However, the Xe isotope tracer provided a robust method for determining small mixtures of young water in nearby and distant wells.

The 1996 tracer experiment was limited in scope and duration. For example, Anaheim Lake only contributes approximately one-third of the annual recharge in the Forebay derived from the recharge basins, and the contribution of Kraemer Basin is equally or even more important because of its higher percolation rate. Furthermore, 6300 acre- $\mathrm{ft}$ is a relatively small amount of recharge water on an annual basis (approximately 5\% of annual basin recharge), and the effects of dispersion and/or dilution potentially overwhelm the tracer with time and distance from the point of recharge. However, significantly larger purchases of Colorado River water $(>10 \%$ annual recharge) for recharge experiments is not necessarily practical for District in most cases. Therefore, it becomes important to have a tracer in the recharge water that can be detected in very small mixtures $(\leq 1 \%$ of the original recharge concentration) in conditions where dispersion has a large effect on tracer transport. As a result, it became evident that a more extensive artificial recharge experiment was needed that recharged water through both Anaheim Lake and Kraemer Basin. In this experiment, unique Xe isotope tracers could be added individually to each basin in order to separate their contribution in recharging groundwater to particular wells. The distinct Xe isotope tracers could also delineate aquifers where recharge water from both recharge basins co-mingled. Furthermore, the monitoring period could be extended to look at transport rates to more distal wells in the Forebay and determine the extent that young water $(<1$ year old) influences recharge to production wells. Also, two separate Anaheim Lake recharge tests could compare different hydrologic conditions and illustrate possible variability in recharge and transport rates.

\subsubsection{Recharge Condition}

A combined Colorado River-Xenon isotope tracer study was initiated in October 1, 1998. Approximately 9500 acre-ft of Colorado River water was diverted and simultaneously recharged into Anaheim Lake and Kraemer Basin. Prior to the tracer study, the basin bottoms were cleaned, which entailed draining water from the basins, scraping fine-grained deposits from the bottom. Miller Basin was left dry during 
Colorado River recharge. Inflow rates to Anaheim Lake and Kraemer Basin were between 85 and 100 cfs. Water depths were 24 feet in both basins when the xenon tracers were added on October $6^{\text {th }}$. During Colorado River recharge, mean daily percolation rates in Anaheim Lake were $2.2 \mathrm{ft} /$ day, and $8.2 \mathrm{ft} /$ day for Kraemer Basin. Approximately half the Colorado River water was recharge in each basin.

Approximately 6 days after the Colorado River water recharge started, a ${ }^{129}$ Xe tracer was introduced into Anaheim Lake. This tracer was introduced using a gas cylinder stationed at the lakeshore and that contained the ${ }^{129} \mathrm{Xe}$ enriched gas. Attached to the cylinder was 1200 feet of standard garden hose extended into the lake to the bottom near the OC-28 discharge point. Near the cylinder the line was split and the second line connected to a sump pump. The pump lifted water from the lake into the hose and mixed with the ${ }^{129} \mathrm{Xe}$-enriched gas as it flowed down the 1200 feet of hose to the lake bottom. It was assumed that the path length of mixing and the pressure maintained in the hose dissolved all the gas into the water before it reached the lake bottom. Approximately nine grams of ${ }^{129} \mathrm{Xe}$ were added to the lake by this method. Samples were not collected from the lake for ${ }^{129} \mathrm{Xe}$ measurements so the starting concentration can only be estimated. For instance, nine grams of ${ }^{129} \mathrm{Xe}$ dissolved in 24 feet of water in Anaheim Lake gives approximately $1.2 \times 10^{-12} \mathrm{mmol}^{129} \mathrm{Xe} / \mathrm{mmol} \mathrm{H}_{2} \mathrm{O}$ (Fig. 16b).

On the same day a ${ }^{136} \mathrm{Xe}$ tracer was added to Kraemer Basin. This tracer was introduced as a single pulse in a central location of the basin over a few hours (Fig. 16c). The same tracer introduction technique used for the 1996 tracer experiment in Anaheim Lake was also used for the ${ }^{136} \mathrm{Xe}$ tracer in Kraemer Basin. The faster percolation rate for Kraemer and its smaller size compared to Anaheim Lake necessitated the rapid introduction of the tracer in order to achieve a high concentration in the recharge water. In particular, approximately four 4-L bottles added a total of approximately 6 grams of ${ }^{136} \mathrm{Xe}$ to the basin water, producing an anticipated dissolved concentration of $1.7 \times 10^{-}$ ${ }^{12} \mathrm{mmol}^{136} \mathrm{Xe} / \mathrm{mmol} \mathrm{H}_{2} \mathrm{O}$. Four samples of basin water were collected within 24 hours after the tracer was released. The average concentration was $6.8 \times 10^{-13} \mathrm{mmol}^{136} \mathrm{Xe} / \mathrm{mmol}$ $\mathrm{H}_{2} \mathrm{O}$ with a standard deviation of $\pm 0.3 \times 10^{-13}$.

By the same reasoning used in the 1996 Anaheim Lake recharge tracer, the atmospheric loss of either ${ }^{129} \mathrm{Xe}$ or ${ }^{136} \mathrm{Xe}$ tracer during the introduction period is likely minimal. ${ }^{129} \mathrm{Xe}$ tracer observed in wells downgradient of Kraemer Basin required the estimated recharge concentration (as opposed to that measured in the basin 24 hours later) in order to achieve a reasonable agreement in mass balance.

\subsubsection{Kraemer Basin Recharge}

Two major flowpaths originating from Kraemer Basin are distinguished based on data from wells west of the basin. The first is a "North Flowpath" which originates from Kraemer Basin and flows west toward wells AM-7, AM-8, SCWC-PLJ2, and A-26 (Fig.18). AM-7 and AM-8 are both monitoring wells cased 210-225 and 268-285 ft bgs, respectively. SCWC-PLJ2 and A-26 are both production wells cased at 402-492 and 266$383 \mathrm{ft}$ bgs, respectively. The second flow path is the "South Flowpath" which originates in Kraemer Basin and flows southwest through OCWD-KB1, AM-10, AM-9, and AM14. All these are monitoring wells and cased respectively at 180-200, 217-235, 285-303, and 297-315 ft bgs. Based on ${ }^{3} \mathrm{H}_{-}{ }^{3} \mathrm{He}$ dating, this latter flowpath appeared to have the highest velocity, since groundwater in AM-14 was only 2 years old (Fig. 7). An 
additional flow component originating from Anaheim Lake flows in the same general direction as Kraemer Basin recharge, with well AM-44 being the furthest downgradient monitoring point before Anaheim Lake recharge water overlaps Kraemer and Miller Basin recharge water. Since Anaheim Lake recharge was labeled with ${ }^{129} \mathrm{Xe}$ it can be distinguished from the ${ }^{136}$ Xe-labeled Kraemer Basin recharge.

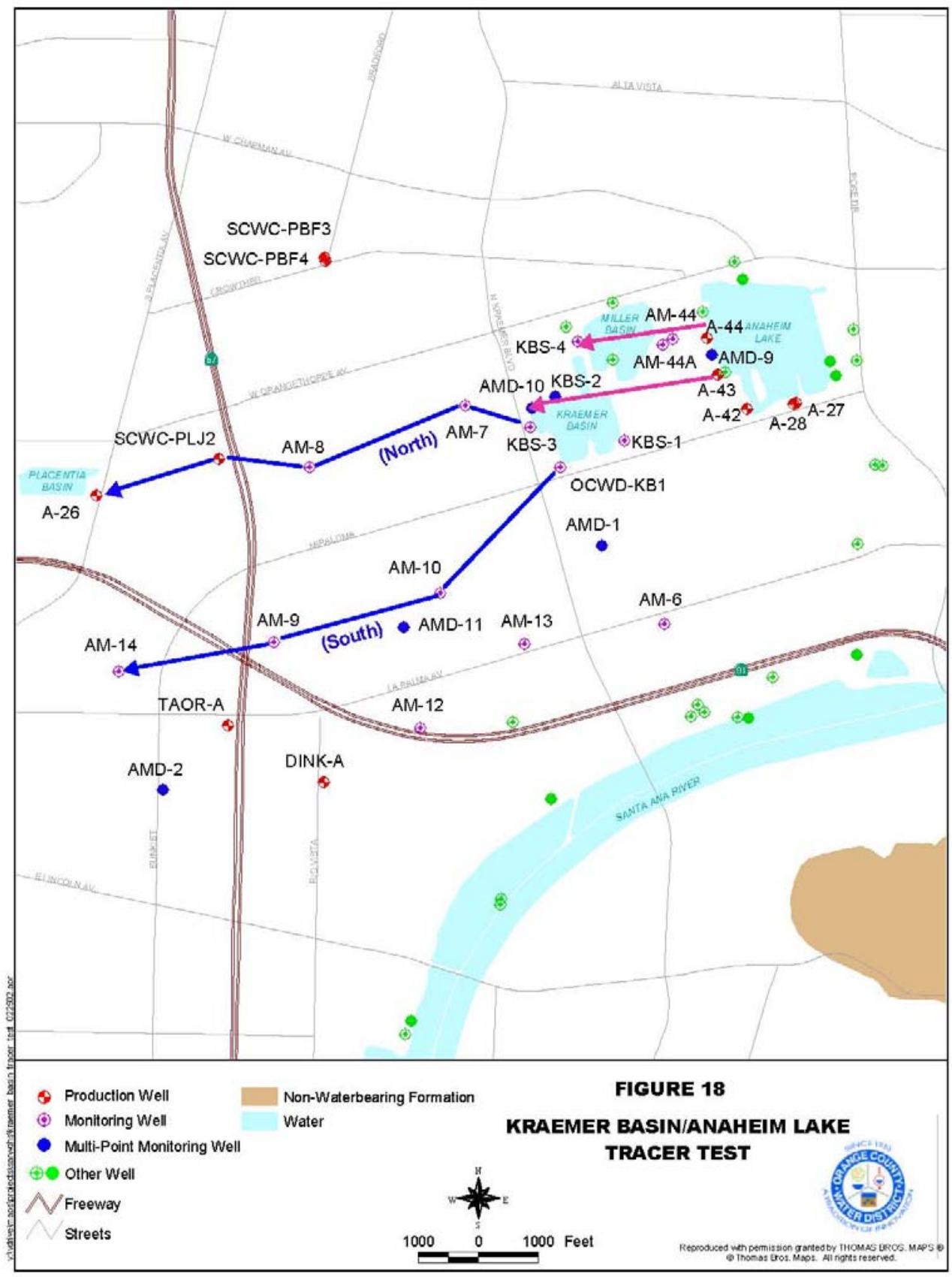

Figure 18. Map of Forebay region showing line of wells used for isotopic breakthrough analysis of tracer pulses arbitrarily named "North", "South", and "Anaheim Lake" flowpaths. 
8.2.2.1 North Flowpath $\delta^{18} \mathrm{O}$ and Xe Isotope Results - The $\delta^{18} \mathrm{O}$ of the Colorado River water was measured in Kramer Basin at the beginning of the tracer experiment (Table. 4a). This $\delta^{18} \mathrm{O}$ value (-11.7 per mil) was similar to that measured in Anaheim Lake water during the 1996 tracer experiment (-11.2 per mil). Clear breakthrough curves of $\delta^{18} \mathrm{O}$ were observed for both monitoring wells AM-7 and AM-8 (Fig. 19a). The $\delta^{18} \mathrm{O}$ value sharply decreased in AM-7 approximately 3 months after the tracer introduction, followed by a more gradual decrease in AM-8 approximately 2-3 months later. At approximately 9 months after the Colorado River water was recharged in Kraemer Basin, a small but measurable decrease in $\delta^{18} \mathrm{O}$ was observed in production well SCWC-PLJ2. A similarly small decrease was suspected for production well A-26 two months later, but follow up data were not available to confirm this $\delta^{18} \mathrm{O}$ trend.
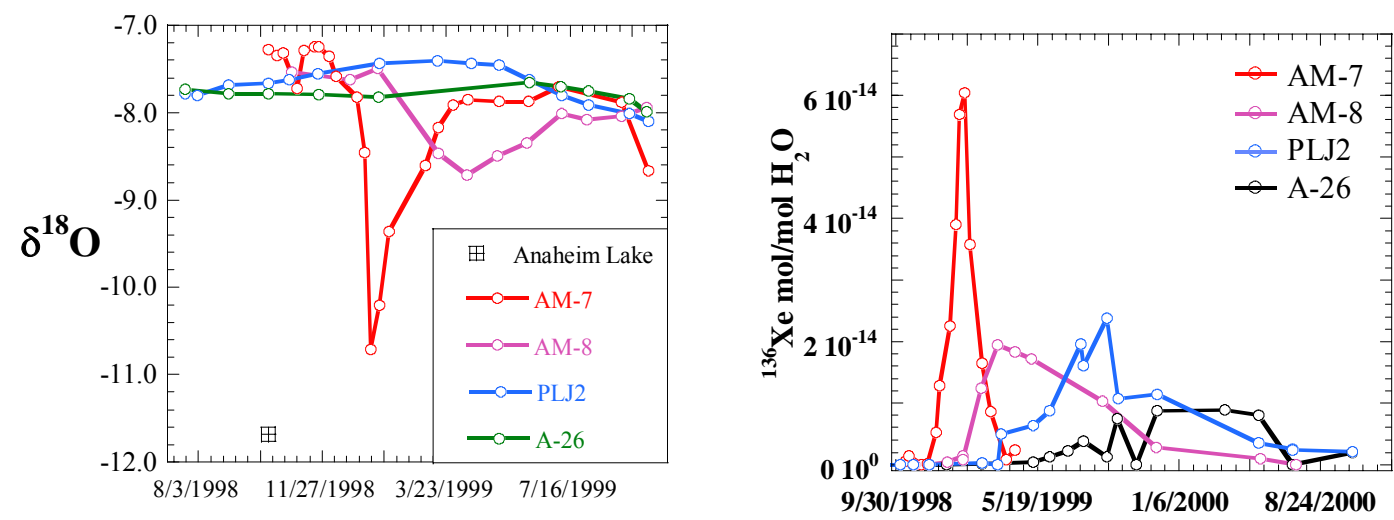

Figure 19. The a) $\delta^{18} \mathrm{O}$ and b) ${ }^{136} \mathrm{Xe}$ tracers showed similar arrival times to wells downgradient of Anaheim Lake forming the "north" flow path.

Although the ${ }^{136} \mathrm{Xe}$ breakthrough in wells AM-7 and AM-8 show a similar arrival time as the $\delta^{18} \mathrm{O}$ (Fig. 19b), the ${ }^{136} \mathrm{Xe}$ tracer was actually detected a couple weeks sooner than the initial arrival of the Colorado River water $\delta^{18} \mathrm{O}$ signature. This occurred because the $\mathrm{Xe}$ isotope measurement is sensitivity to approximately a $1 \%$ change in relative concentration, whereas sensitivity in the $\delta^{18} \mathrm{O}$ measurement is poorer and its relative change depended on a small difference between SAR and Colorado River water abundance.

Only AM-7 showed a relatively symmetrical breakthrough curve for 136Xe, whereas AM-8 had a long tail and SCWC-PLJ2 and A-26 clearly had multiple peaks. These latter two are production wells with multiple screen depths. Integration of the 136Xe data with time for AM-7 results in a total concentration of $2.7 \times 10^{-12} \mathrm{mmol}^{136} \mathrm{Xe} / \mathrm{mmol}_{2} \mathrm{O}$. This is approximately 1.6 times greater than the concentration estimated on a basis of mass per basin water volume, and four times greater than the concentration measured in the basin 24 hours after tracer introduction. The integrated concentration for the AM-8, SCWCPLJ2, and A-26 range between 1.4 and 2.8 times greater than the concentration estimated by mass and basin volume. These results suggest little of the tracer introduced was lost to the atmosphere. 
The ${ }^{136}$ Xe breakthrough for SCWC-PLJ2 occurred 8-9 months after Colorado River was recharged in Kraemer Basin. This is consistent with the $\delta^{18} \mathrm{O}$ decreases noted at the same time in this well (Fig. 19a). The maximum concentration of ${ }^{136} \mathrm{Xe}$ detected was $<2 \%$ of the concentration measured in Kraemer Basin, and was similar to well AM-8. Most of the detectable ${ }^{136} \mathrm{Xe}$ tracer passed through well SCWC-PLJ2 within about 6 months.

Approximately 3.5 months after ${ }^{136} \mathrm{Xe}$ was detected in SCWC-PLJ2, it arrived in well A-26, which is approximately 2000 feet downgradient. The maximum ${ }^{136} \mathrm{Xe}$ detected was at or slightly above $0.5 \%$ of the concentration measured in Kraemer Basin and was persistent for about 8 months.

One groundwater sample from well AM-29, $>3000$ feet downgradient of A-26, had a ${ }^{136} \mathrm{Xe}$ concentration at $\geq 0.5 \%$ of the Kramer Basin concentration. It was detected 21 months after the Colorado River water was recharged in Kraemer Basin and approximately 10 months after ${ }^{136} \mathrm{Xe}$ was detected in A-26. AM-29 has a perforation interval of 340-358 $\mathrm{ft}$ bgs, which overlaps with the perforation of A-26 (266-383 ft bgs). The total distance between the center of Kraemer Basin and AM-29 is approximately 2.2 miles. The implication of ${ }^{136} \mathrm{Xe}$ tracer in AM-29 is that a linear transport rate of $>6000$ feet year is required to travel this distance from Kraemer Basin.

8.2.2.2 South Flowpath $\delta^{18} O$ and Xe Isotope Results - The $\delta^{18} \mathrm{O}$ breakthrough in monitoring well OCWD-KB1 was within 15 days of the initial Colorado River water recharge in Kraemer Basin (Fig. 20a). At maximum breakthrough, the $\delta^{18} \mathrm{O}$ value matched that measured for COR in Kraemer Basin. Downgradient, the $\delta^{18} \mathrm{O}$ showed a muted breakthrough at monitoring well AM-10 approximately 4 to 5 months after Colorado River water recharge began, with a total change in $\delta^{18} \mathrm{O}$ from -7.5 to -8.3 per mil. A $\delta^{18} \mathrm{O}$ value between -8.3 and -8.0 per mil persisted in this well through September, 1999. A similar muted breakthrough was seen for monitoring well AM-9, with the arrival occurring approximately 4 months after AM-10. No perceptible change in the $\delta^{18} \mathrm{O}$ value toward Colorado River was noted in AM-14 during the observation period.
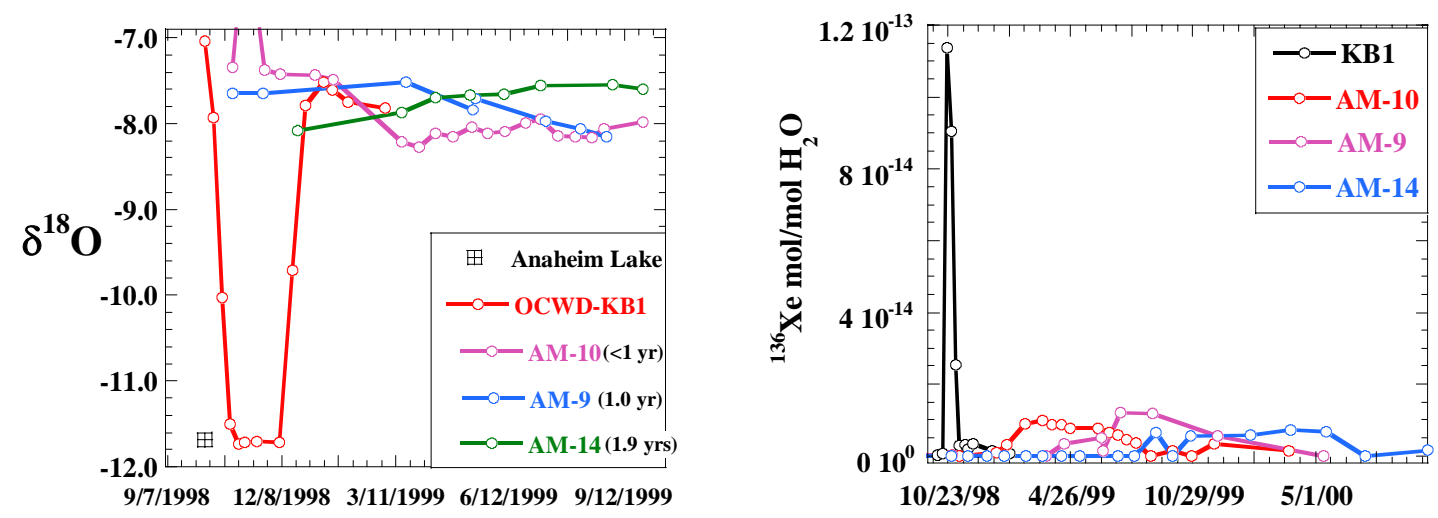

Figure 20. The a) $\delta^{18} \mathrm{O}$ and b) ${ }^{136} \mathrm{Xe}$ tracers showed similar arrival times to wells downgradient of Anaheim Lake forming the "south" flow path. Dispersion greatly decreases tracer concentration during downgradient flow. 
The ${ }^{136}$ Xe tracer was initially detected in OCWD-KB1 15 days after Colorado River water recharge started and 11 days after the ${ }^{136} \mathrm{Xe}$ was added (Fig. 20b). The initial detection was also the highest concentration observed, and was approximately $7 \%$ of the concentration measured in Kramer Basin. The ${ }^{136} \mathrm{Xe}$ decreased in OCWD-KB1 to near detection limits within approximately 30 days after the Colorado River water recharge started. An integrated ${ }^{136} \mathrm{Xe}$ concentration in OCWD-KB1 over time was essentially the same as that estimated on a mass per water volume basis.

Like the $\delta^{18} \mathrm{O}$, the ${ }^{136} \mathrm{Xe}$ in well AM-10 also arrived approximately 4 to 5 months after Colorado River water recharge began in Kraemer Basin, and also had a muted breakthrough concentration compared to the initial concentration in the recharge water. The maximum detected ${ }^{136} \mathrm{Xe}$ concentration in $\mathrm{AM}-10$ was $<1 \%$ of the concentration measured in Kraemer Basin. However, this low concentration persisted in AM-10 for 4 to 5 months before it decreased below the detection limit.

Downgradient in AM-9, the ${ }^{136} \mathrm{Xe}$ arrived approximately 8 months after Colorado River water recharge began and 3 to 4 months after it was observed in AM-10. AM-9 and AM-10 are separated by approximately 2700 feet. Like AM-10, the peak ${ }^{136} \mathrm{Xe}$ concentration in well AM-9 was $<1 \%$ of the recharge concentration. This low ${ }^{136} \mathrm{Xe}$ concentration persisted in AM-9 for nearly 9 months.

In October $1999,{ }^{136} \mathrm{Xe}$ was detected in AM-14 at approximately $<1 \%$ of the recharge concentration. This suggests a linear travel velocity to AM-14 from Kraemer Basin of about one year. The ${ }^{136} \mathrm{Xe}$ concentration never exceeded $0.4 \%$ of the recharge source for this well. The ${ }^{136}$ Xe persisted in AM-14 for approximately 7 months.

Two analyses of well AM-15, about $3100 \mathrm{ft}$ downgradient of AM-14 and approximately 12,000 feet from Kramer Basin, suggested low levels of ${ }^{136} \mathrm{Xe}$ at $<1 \%$ of the recharge concentration. These were measured in July and October 2000, suggesting a linear transport rate from Kramer Basin of about 6000 feet per year.

8.2.2.3 $\delta^{18} \mathrm{O}$ and Xe Isotope Results from Wells adjacent to Kramer Basin - Breakthrough of Colorado River water was observed with $\delta^{18} \mathrm{O}$ and ${ }^{136} \mathrm{Xe}$ in several other wells in close proximity of Kraemer Basin. These include monitoring wells KBS-1, KBS-2 level 1 and level 2, KBS-3, and AMD-10.

The $\delta^{18} \mathrm{O}$ measurements showed that KBS-1 had four separate breakthrough curves over a five and half month period following Colorado River water recharge into Kraemer Basin (Fig. 21ab). Only ${ }^{136} \mathrm{Xe}$ was detected in this well, indicating that each breakthrough originated from Kraemer Basin. Note KBS-1 is located on the eastside of Kraemer Basin, just slightly upgradient. Recharge to this well is likely strongly influenced by eastward progression of a recharge mound developed beneath Kramer Basin. 

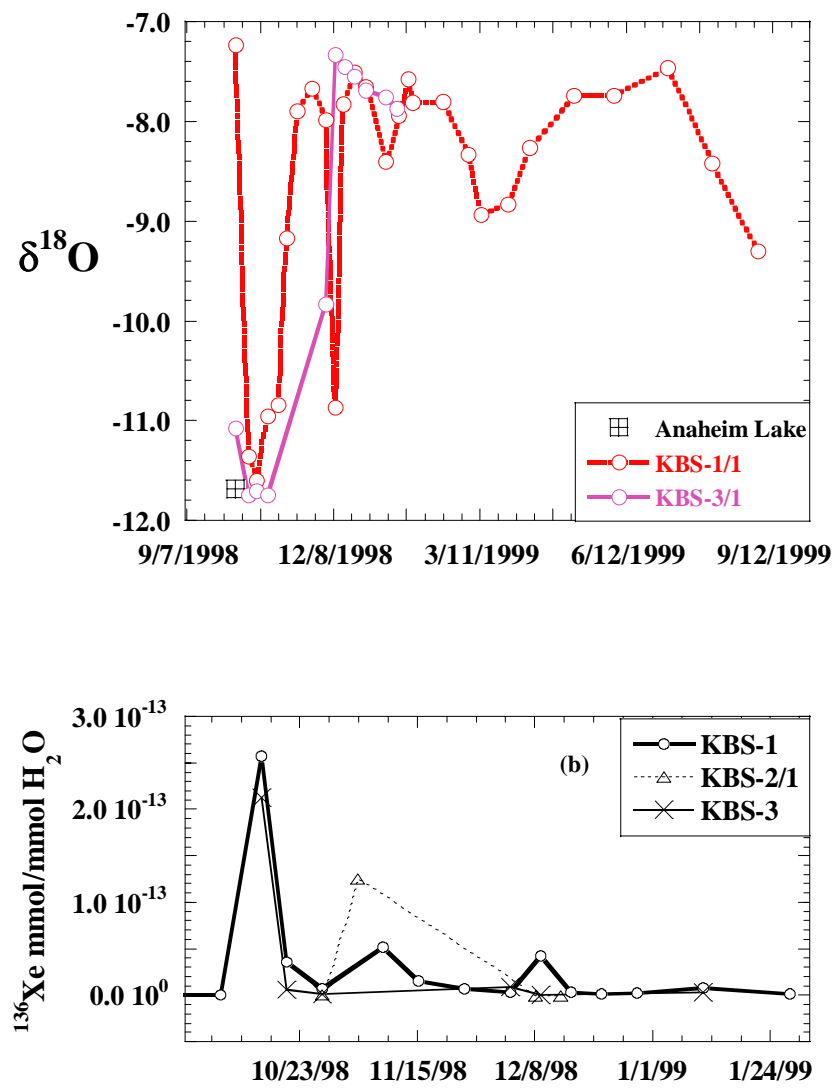

Figure 21. The a) $\delta^{18} \mathrm{O}$ values and b) ${ }^{136} \mathrm{Xe}$ tracer data show rapid transport to these nearby wells. At least three different arrivals occur in well KBS-1, suggesting multiple flow paths.

The $\delta^{18} \mathrm{O}$ also indicated that Colorado River water arrived in KBS-2 level 1 and KBS3 in $<7$ days, and returned to an SAR water signature within approximately 2 months. The ${ }^{136} \mathrm{Xe}$ showed a similar effect as the $\delta^{18} \mathrm{O}$. In groundwater sampled from KBS-2 level 2 , the $\delta^{18} \mathrm{O}$ breakthrough occurred approximately 2 months after Colorado River water recharge began in Kraemer Basin (Table 4a). The $\delta^{18} \mathrm{O}$ was a Colorado River value about 2 weeks later, indicating the water originated solely from Colorado River water recharge. KBS-2 level 2 is approximately $200 \mathrm{ft}$ bgs. Only one analysis for ${ }^{136} \mathrm{Xe}$ was performed on KBS-2 level 2. This was for a sample collected in early December 1998, which had a ${ }^{136} \mathrm{Xe}$ concentration approximately $2 \%$ of the recharge water.

Monitoring well AMD-10 is a well with five nested levels ranging from approximately 200 feet below the surface for level 1 to $>1000$ feet for level 5. This well was completed in early 1998 to monitor water quality and water level responses to Kraemer Basin and Anaheim Lake recharge. Well AMD-10 was also extensively sampled during the 1998 tracer experiments (Fig. 22ab). The $\delta^{18} \mathrm{O}$ results indicate that Colorado River water arrived at the first level approximately 10 weeks after recharge started, and the maximum Colorado River isotopic value observed was only $36 \%$ of the recharge water $\delta^{18} \mathrm{O}$ value. 
The $\delta^{18} \mathrm{O}$ in the $2^{\text {nd }}$ level of AMD-10 began to decrease in June 1999, approximately 9 months after Colorado River water recharge began. The Colorado River signature of level 2 groundwater did not exceed $34 \%$ of the recharge water. No significant decrease in $\delta^{18} \mathrm{O}$ was observed in levels 3 through 5 that would indicate Colorado River water arrival. However, for groundwater in level 4 , the $\delta^{18} \mathrm{O}$ averaged -8.4 per mil \pm 0.1 , suggesting approximately a $30 \%$ mixture of Colorado River water with SAR water. Note that in level 4, the $\delta^{18} \mathrm{O}$ does not form a breakthrough curve, but rather had this lower $\delta^{18} \mathrm{O}$ value from the beginning of its monitoring and persisted throughout the observation period.
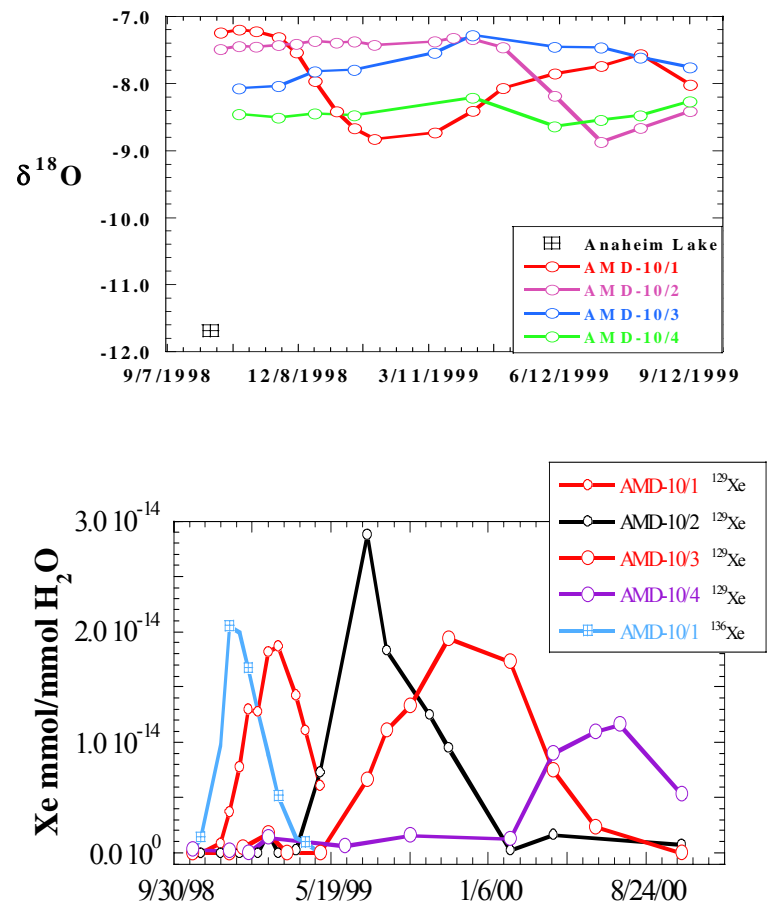

Figure 22. The a) $\delta^{18} \mathrm{O}$ of levels $1-4$ of AMD-10 suggests recharge from the basins, while b) the xenon isotopes indicate that most of the recharge is derived from Anaheim Lake due to the occurrence of ${ }^{129} \mathrm{Xe}$.

The ${ }^{136} \mathrm{Xe}$ was only observed in AMD-10 level 1, arriving approximately 2 months after Colorado River water recharge began and reaching a maximum concentration of $1.2 \%$ of the recharge water estimated for Kraemer Basin. However, ${ }^{129} \mathrm{Xe}$ was detected in levels one through four of AMD-10, indicating that this well primarily received Anaheim Lake recharge. The ${ }^{129} \mathrm{Xe}$ arrived in level one approximately two weeks after the ${ }^{136} \mathrm{Xe}$ was first observed, and showed a maximum concentration of $2.4 \%$ of the estimated Anaheim Lake recharge concentration $\left(1.2 \times 10^{-12} \mathrm{mmol}^{129} \mathrm{Xe} / \mathrm{mmol}_{2} \mathrm{O}\right)$. In May 1999, the ${ }^{129} \mathrm{Xe}$ arrived in level 2, slightly earlier than observed for the $\delta^{18} \mathrm{O}$. Approximately 2 months later the ${ }^{129} \mathrm{Xe}$ was observed in level 3. The ${ }^{129} \mathrm{Xe}$ was first observed in level 4 in April 2000. The ${ }^{129}$ Xe showed similar peak shapes for all four levels, and maximum breakthrough concentration $<3 \%$ of the estimated recharge concentration. In addition, ${ }^{124} \mathrm{Xe}$ was observed in level 4 of AMD-10 in October 1998, 24 months after the 1996 
Anaheim Lake recharge experiment began (Table $4 \mathrm{~b})$. The ${ }^{124} \mathrm{Xe}$ decreased after this initial detection. ${ }^{129} \mathrm{Xe}$ was detected in level four 19 months after the beginning of the 1998 tracer experiment, which is similar to the 24 month travel time computed for ${ }^{124} \mathrm{Xe}$.

\subsubsection{Anaheim Lake Recharge}

The $\delta^{18} \mathrm{O}$ value of Anaheim Lake water was not measured for the 1998 recharge study, however, it was measured in Kraemer Basin and is assumed to be the same. Breakthrough curves in wells A-27, A-28, and AMD-9 level 1 for the 1998 Colorado River water $\delta^{18} \mathrm{O}$ tracer had striking similarities to those observed in the 1996 tracer experiment (Table 4a; Fig. 23a). In particular, the duplication of the 15-30 day arrival and turnover time for A27 and AMD-9 level 1 and the attenuated breakthrough of A-28 demonstrate that hydrologic conditions were similar for these nearby wells for both tracers experiments. In contrast, the $1998 \delta^{18} \mathrm{O}$ arrival of the Colorado River water in well AM-44 approximately 1000 feet down gradient was around one month sooner ( 2 month arrival) than in 1996 (3 month arrival). The differences in hydrologic conditions between these two recharge experiments, aside from groundwater pumping, was that Miller Basin was full during the 1996 test, but empty during the 1998 experiment. It is hypothesized that groundwater recharge from Miller Basin raised groundwater levels in this vicinity during the 1996 tracer test, which reduced the hydrologic gradient between Miller and Anaheim basins and reduced the velocity of Anaheim Lake recharge towards well AM-44.
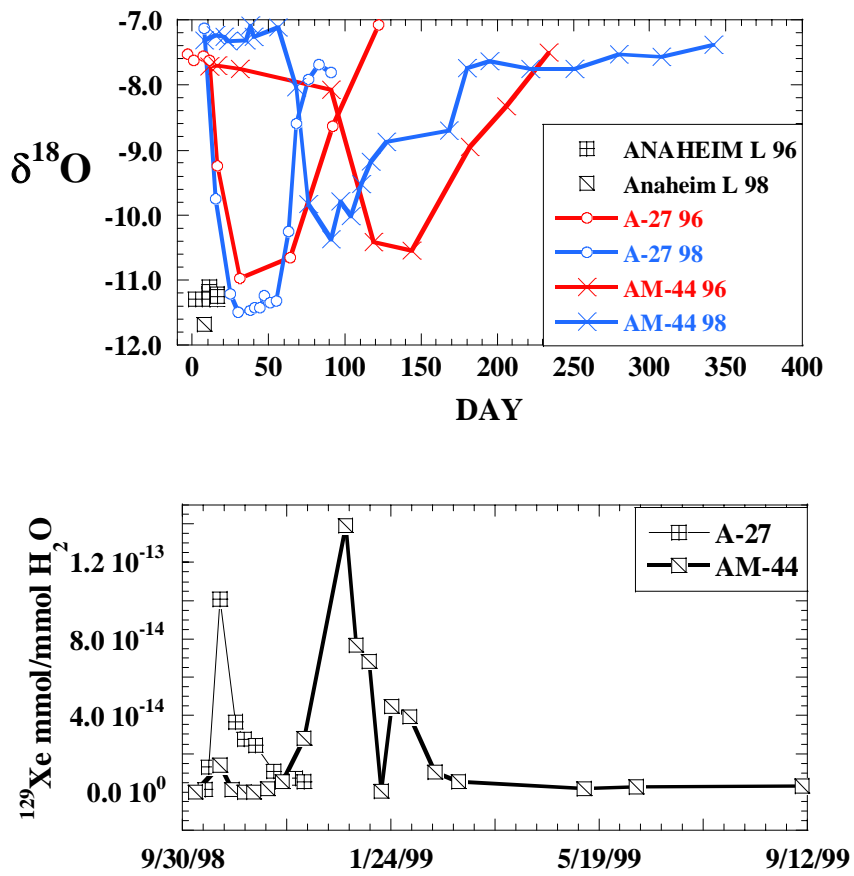

Figure 23. The a) $\delta^{18} \mathrm{O}$ tracer of Anaheim Lake recharge was similar to the 1996 recharge experiment, except for AM-44, which showed a faster velocity in 1998 . b) The ${ }^{129} \mathrm{Xe}$ tracer showed similar arrival times as the $\delta^{18} \mathrm{O}$.

The ${ }^{129} \mathrm{Xe}$ was not directly measured in Anaheim Lake. The initial concentration was estimated from the mass of the ${ }^{129} \mathrm{Xe}$ added to the lake divided by the volume of stored 
water at the time the tracer was introduced. The initial concentration was estimated at $1.2 \times 10^{-12} \mathrm{mmol}^{129} \mathrm{Xe} / \mathrm{mmolH}_{2} \mathrm{O}$. The ${ }^{129} \mathrm{Xe}$ was detected in A-27 and AM-44 at the same time as the $\delta^{18} \mathrm{O}$ (Fig. 23b). The maximum breakthrough concentration observed in AM44 was approximately $12 \%$ of the concentration estimated for Anaheim Lake. Note that the highest ${ }^{129} \mathrm{Xe}$ concentration detected in AM-44 was somewhat greater than in A-27. This could suggest that the peak breakthrough concentration in A-27 was not detected.

Colorado River water breakthrough occurred in well KBS-4 approximately 4 months after recharge began (Table 4a). The starting $\delta^{18} \mathrm{O}$ value before breakthrough was unusually high at -6.5 per mil, and the breakthrough showed a decrease to only about 7.8 per mil. The shift was significant enough to indicate Colorado River water arrival. Subsequent recharge of Colorado River water into Anaheim Lake for operational purposes occurred several months after the initiation of the 1998 tracer study. The Colorado River water recharge pulse from this later diversion can also be seen in the KBS-4 data around March of 1999, which has a much stronger breakthrough. The ${ }^{129} \mathrm{Xe}$ was observed only once in KBS-4, approximately 4 months after the October 1998 recharge in Anaheim Lake began. This ${ }^{129} \mathrm{Xe}$ detection is consistent with the $\delta^{18} \mathrm{O}$ decrease indicating a Colorado River water source.

Detection of ${ }^{129} \mathrm{Xe}$ in monitoring well AMD-11 level 2 in October 2000 suggested that Anaheim Lake water recharged this well in about a two-year timeframe. The ${ }^{129} \mathrm{Xe}$ was not detected in any of the other levels. In addition, neither the ${ }^{124} \mathrm{Xe}$ nor the ${ }^{136} \mathrm{Xe}$ was observed in any of the different levels of AMD-11.

\subsection{Santa Ana River Tracer Study}

Introducing a tracer to water in a recharge basin required a single spike of isotopically enriched xenon gas that once introduced at depth, spread and mixed laterally and recharged into the groundwater basin at a relatively fast rate (2-8 feet per day). For the $\mathrm{SAR}$, the hydrologic conditions required a different approach. The river is a dynamic stream with a shallow depth and has a low water volume on a daily basis compared to the basins (i.e. 100s acre-ft per day for SAR versus 1000s of acre- $\mathrm{ft}$ per day for Anaheim Lake or Kraemer Basin). Furthermore, the shallow depth and moderate turbulence of the SAR would promote significant loss of any dissolved gas tracer, which for an isotopically enriched xenon gas it would be prohibitively expensive to label several days of SAR water flow. Therefore, an inexpensive tracer that could be dissolved in the river water and subsequently detected at low concentrations in groundwater was needed to successfully trace SAR recharge.

In the summer of 1998 , sulfur-hexafluoride $\left(\mathrm{SF}_{6}\right)$ was chosen as a tracer for the SAR (see Gamlin et al. 2001 for additional discussion). Sulfur-hexafluoride does not occur naturally in the environment. It is inexpensive and can be detected at low concentration (see Appendix 1). Approximately 3000 acre-ft of SAR were labeled with $\mathrm{SF}_{6}$ using three injectors discharging $\mathrm{SF}_{6}$ gas $\left(99.8 \%\right.$ purity) directly into the river at $20 \mathrm{~cm}^{3}$ per minute over a period of 15 days. $\mathrm{SF}_{6}$ was introduced into SAR water only within the channel over an approximately five and half mile length starting just below the Imperial Headgates inflatable dam (Fig. 24). No $\mathrm{SF}_{6}$ was introduced into the off-river recharge basins. The $\mathrm{SF}_{6}$ was introduced during baseflow with a mean discharge rate of 100 cubic feet per second (cfs). The percolation rate of the river channel was equal to the discharge 
rate of $100 \mathrm{cfs}$, such that all of the water tagged with $\mathrm{SF}_{6}$ percolated into the ground, excluding minor evaporative losses.

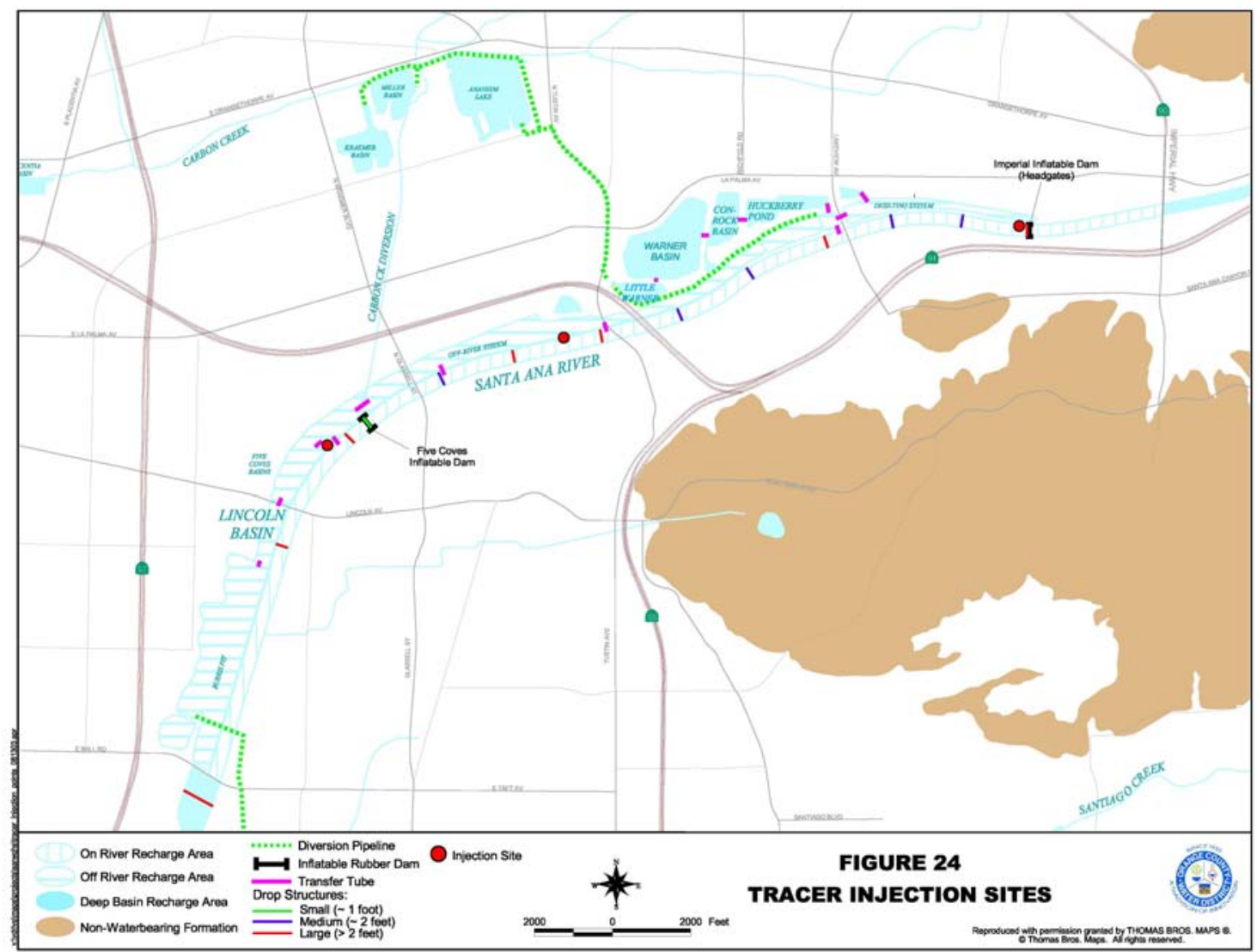

Figure 24. Map of SAR and locations of $\mathrm{SF}_{6}$ tracer introduction and large drop structures contributing to turbulent surface water flow.

The $\mathrm{SF}_{6}$ concentration measured in the river channel varied over a factor of 10 (Fig. 25). This was due to the presence of three drop structures along the channel, contributing to turbulence and enhancing atmospheric loss. Nevertheless, even the minimum concentration detected in the river was nearly 100 times greater than the detection limit of the $\mathrm{SF}_{6}$ analysis, which provided an easily detected tracer signal in groundwater even when the tracer was relatively dispersed. However, the variable recharge concentration limits confidence in mixing calculations. 


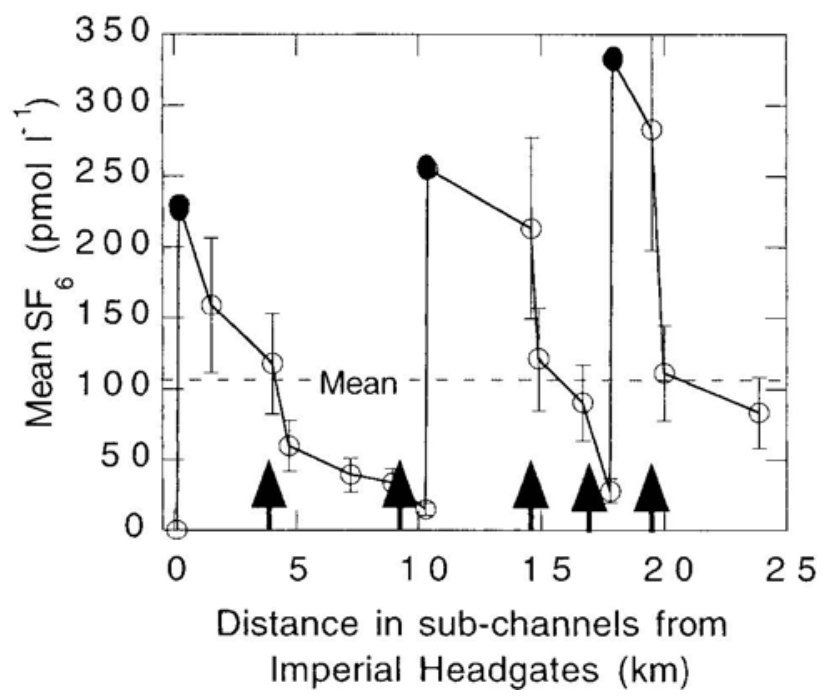

Figure 25. Mean concentrations of $\mathrm{SF}_{6}$ in the SAR along the length of tracer introduction and recharge. Note the approximate order of magnitude change in concentration associated with drop structures. Reproduced from Gamlin et al. (2001).

Like the basin recharge experiments, several wells were selected as monitoring points for tracer arrival and duration (Fig. 7). The wells closest to the river channel were WBS-4, SAR-8, SAR-7, SAR-6, OCWD-FH1, OCWD-FC1, and OCWD-LV1. The timing and frequency of each sampling point was chosen based on the previous age-dating work. Wells farther from the river channel were chosen as sampling points later as the $\mathrm{SF}_{6}$ pulse transported away from the river, and they are listed along with the $\mathrm{SF}_{6}$ results in Table 5.

Earliest arrival of the $\mathrm{SF}_{6}$ tracer was observed in shallow wells WBS-4 level $1(\sim 70 \mathrm{ft}$ bgs) and SAR-8 level 1 ( $\sim 5 \mathrm{ft}$ bgs), where breakthrough occurred within one and three weeks, respectively, after the river was spiked (Fig. 26a). Note that even though the $\mathrm{SF}_{6}$ arrived first in WBS-4 level 1, its concentration is nearly ten times lower than in SAR-8 level 1. It is possible that high concentrations similar to SAR-8 level loccurred in WBS-4 level 1 , but they were not captured by the sample frequency represented in the data.
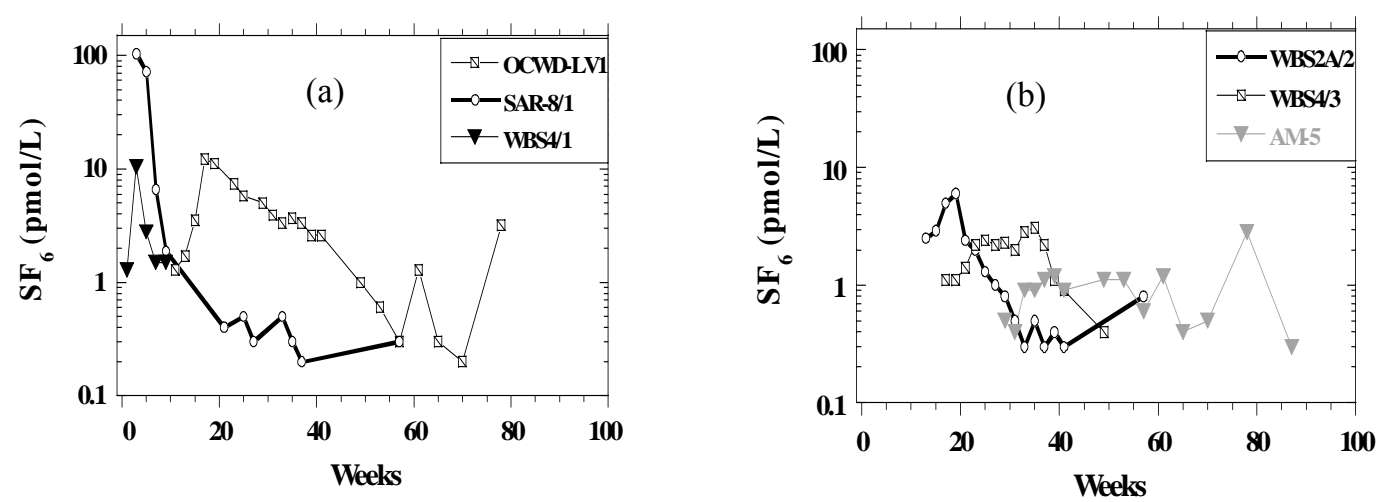

Figure 26a and 26b. Breakthrough curves for $\mathrm{SF}_{6}$ tracer in wells recharged by the SAR.

The $\mathrm{SF}_{6}$ arrival in the other wells adjacent to the SAR did not occur until after nine weeks after $\mathrm{SF}_{6}$ introduction was initiated. For example, in well OCWD-LV1 


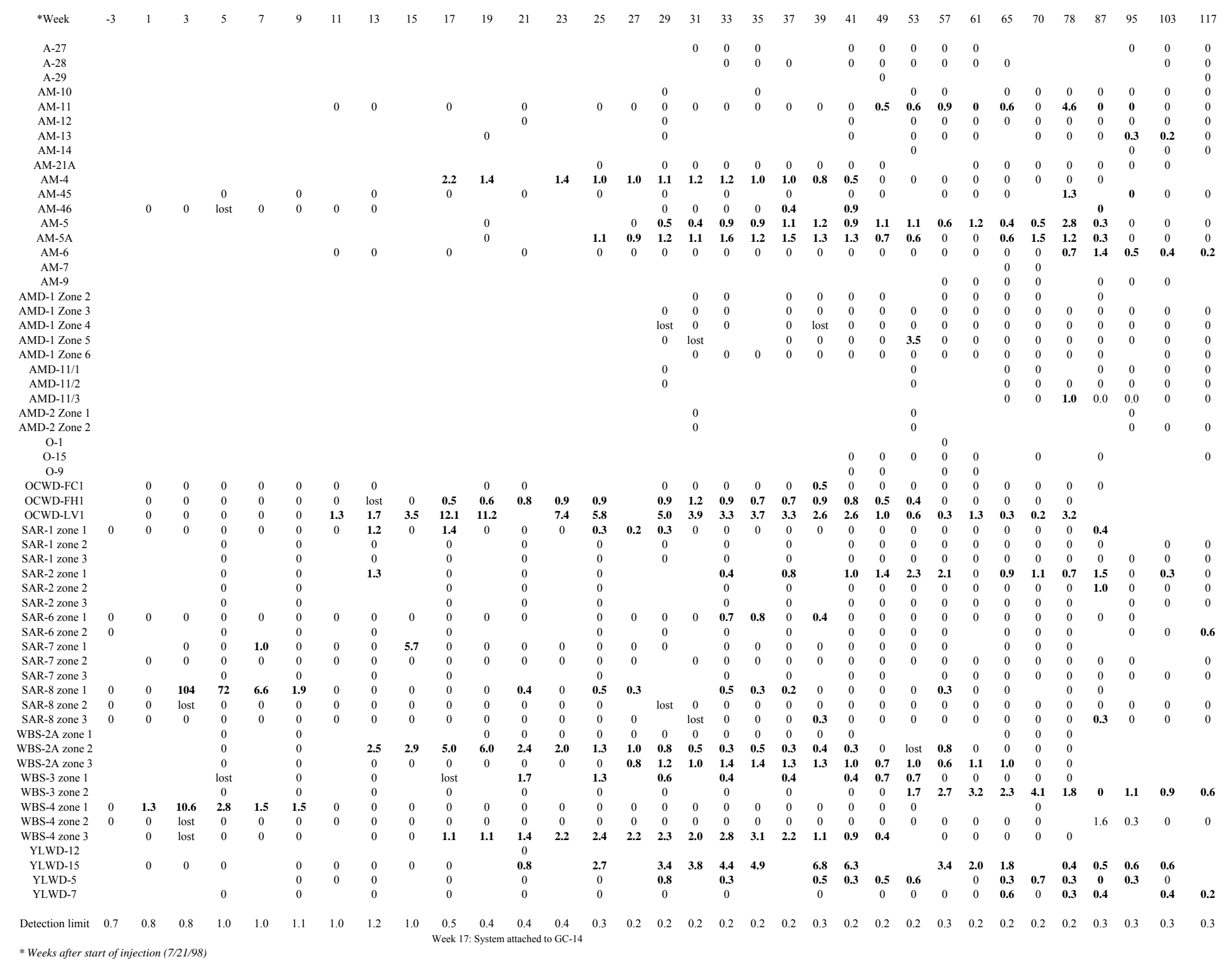


(approximately 145 feet below the surface), $\mathrm{SF}_{6}$ was measured at approximately one $\mathrm{pmol} / \mathrm{L}$ concentration on week 11, and by week 17 was measured at a peak concentration of approximately $12 \mathrm{pmol} / \mathrm{L}$ (Fig. 26a). Note that this concentration was lower than the lowest concentration measured in the SAR. In fact, river recharge to OCWD-LV1 is upgradient of any drop structures, and the $\mathrm{SF}_{6}$ concentration of recharge in this area was likely $>100 \mathrm{pmol} / \mathrm{L}$. The total tracer duration in OCWD-LV1 was $>70$ weeks.

A similar breakthrough to OCWD-LV1 was observed in well WBS-2a (level 2), but the tracer duration was $<32$ weeks (Fig. 26b). In the deeper sampling point of WBS-4 (level 3; approximately $215 \mathrm{ft}$ bgs) the $\mathrm{SF}_{6}$ breakthrough occurred at week 17 and persisted at $<10 \mathrm{pmol} / \mathrm{L}$ for around 32 weeks. Note that the $\mathrm{SF}_{6}$ was not observed at the shallower depth in WBS-4 (level 2) until week 95 at around 2 pmol/L. This trend is consistent with the ${ }^{3} \mathrm{H}-{ }^{3} \mathrm{He}$ ages, where the groundwater in the second level of WBS-4 was 19 years old compared to less than one year old in the third level.

The $\mathrm{SF}_{6}$ arrived at the more distal well AM-5 on week 29 and persisted through week 87 at approximately $1 \mathrm{pmol} / \mathrm{L}$ concentration. Note that AM-5 is also downgradient of Warner Basin, which intentionally did not receive SAR water tagged with $\mathrm{SF}_{6}$ tracer. All other wells where $\mathrm{SF}_{6}$ was observed typically had tracer arrival after week 13 and none had $\mathrm{SF}_{6}$ concentration in excess of $10 \mathrm{pmol} / \mathrm{L}$ (see Table 5).

\section{Discussion on Sources of Recharge}

Williams (1997) identified groundwater in the Orange County basin on the basis of the $\delta \mathrm{D}$ and $\delta^{18} \mathrm{O}$ values and separated them into different recharge sources. In all cases groundwater was separated into "local", "native", "Colorado River", or "recent" recharge designations (Fig. 9). The latter two designations are related to groundwater recharged from modern diversion projects in the Forebay. Colorado River water has unusually low stable isotope values because of its mid-continent origin, and plots below the global meteoric water line due to extensive evaporation. The "recent" SAR recharge has higher stable values because of its more local origin and also lies below the global meteoric water line because of evaporation. "Local" recharge originates from precipitation infiltrating locally in the Chino Hills and the Santa Ana Mts., and is characterized by

higher $\delta \mathrm{D}\left(>-60\right.$ per mil) and $\delta^{18} \mathrm{O}$ (typically $>-8.5$ per mil) values. This recharge source tends to plot near the meteoric water line. "Native" recharge is groundwater originating from SAR infiltration before agricultural and urban development ( $>75$ years ago) in the area and is usually lower than -8.5 per mil $\delta^{18} \mathrm{O}$, and also plots near the meteoric water line.

Two groundwater samples collected and measured during the first phase of the LLNL study can be easily placed into "native" and "local" recharge groups. Well A-47 falls into a "native" recharge group by virtue of its $\delta \mathrm{D}$ and $\delta^{18} \mathrm{O}$ values of -60 and -8.5 per mil, respectively, its low initial ${ }^{3} \mathrm{H}$ content of $40 \mathrm{pCi} / \mathrm{L}$, which indicates significant dilution with non-tritiated water, and its relatively low ${ }^{14} \mathrm{C}$ content of $84 \mathrm{pmc}$. Well A-47 has a perforation interval $>900$ feet long (482-1375 feet below the surface) and is located several hundred feet downgradient of well CB-1, whose groundwater collected in level 6 had a similar $\delta^{18} \mathrm{O}$ and initial ${ }^{3} \mathrm{H}$ as $\mathrm{A}-47$. It is concluded that $\mathrm{A}-47$ groundwater predominately consists of native groundwater recharge from ancient SAR infiltration before the recharge basins were developed. 
Well F-AIRP is located to the north of A-47 and just south of the Coyote Hills. Its $\delta \mathrm{D}$ and $\delta^{18} \mathrm{O}$ (-52 and -7.9 per mil, respectively) fall within the local recharge group defined by Williams (1997) and lies approximately on the global meteoric water line. This well water also had a low ${ }^{14} \mathrm{C}$ content of $62 \mathrm{pmc}$ and a measurable radiogenic ${ }^{4} \mathrm{He}$ content, which together both indicate older groundwater of hundreds to thousands of years. The location of F-AIRP south of the Coyote Hills suggest that its recharge originates predominately from this area and is not influenced by recharge practices in the basins or from the SAR.

It is useful to compare the stable isotope values of "native" and "local" recharge to that of well A-42 (Fig 9). Note that the $\delta \mathrm{D}$ and $\delta^{18} \mathrm{O}$ value of A-42 lie between that of A-47 and F-AIRP. Note also that A-42 does not plot similar to those wells influenced by "recent" SAR recharge such as HG-1, YLWD-11, YLWD-5, and YLWD-15. Instead, A42 plots near the global meteoric water line close to the "local" recharge characterized by F-AIRP.

In comparison, well A-43, whose construction and location are similar to A-42, plots in the same group as wells HG-1, YLWD-11, YLWD-15, and YLWD-5. These wells plot below the meteoric water line in the same general area as modern SAR. Note that several SAR isotopic analyses have a relatively large variation, but in most cases, except when collected after a rainstorm (March, 1995), they all plot below the meteoric water line. The region in which A-43, YLWD-11, YLWD-5, YLWD-15, and HG-1 plot are consistent with an evaporated SAR water. The $\delta^{18} \mathrm{O}$ value of unevaporated SAR is likely less than -9.0 per mil. This value is consistent with precipitation and runoff from the upper SAR watershed (Williams and Rodoni, 1997). The approximately 11 year old ${ }^{3} \mathrm{H}-$ ${ }^{3} \mathrm{He}$ age for both A-43 and YLWD-5 suggests that modern SAR water, which is dominated by evaporated upper SAR watershed runoff, has recharged these wells consistently over this time period.

The dissimilarity of A-42 isotopic composition to A-43 suggests that recharge sources and flow dynamics toward the deep wells below Anaheim Lake are complex. This complexity may be due to the variable recharge sources in the area and the changing head conditions due to basin operations. A-42 furthermore has a ${ }^{3} \mathrm{H}-{ }^{3} \mathrm{He}$ age (18 years) greater than A-43, suggesting recharge source to these wells may be slowly changing over time.

It is tempting to interpret the A-42 stable isotope values as strictly a "local" recharge signature, however, the calculated initial ${ }^{3} \mathrm{H}$ value is $110 \mathrm{pCi} / \mathrm{L}$, which is too high for a locally recharged groundwater $<20$ years old (Table 6). Note AMD-9 level 4 had a $\delta^{18} \mathrm{O}$ value of -7.73 per mil, a ${ }^{3} \mathrm{H}-{ }^{3} \mathrm{He}$ age of 25 years old, and an initial ${ }^{3} \mathrm{H}$ of $173 \mathrm{pCi} / \mathrm{L}$. This initial ${ }^{3} \mathrm{H}$ nearly matches that expected in weighted precipitation at Santa Maria, CA. Therefore, groundwater in AMD-9 level 4 appears to be recharged exclusively by either SAR or by local recharge.

In contrast, A-42 has a slightly lower $\delta^{18} \mathrm{O}$ value of -8.2 per mil. This slightly lower value could suggest that the groundwater is a mixture of "local" recharge and Colorado River water. For example, if $10 \%$ of the groundwater in A-42 were Colorado River water $\left(-11.5\right.$ per mil), and $90 \%$ was the same as F-AIRP, then the mixed $\delta^{18} \mathrm{O}$ value would be around -8.3 per mil. This small mixture would only represent a small shift from the "local" recharge source water plotted in figure 9. In order to match the high initial ${ }^{3} \mathrm{H}$ of $110 \mathrm{pCi} / \mathrm{L}$ observed in A-42, the $10 \%$ Colorado River water mixed in this well would require an initial ${ }^{3} \mathrm{H}$ concentration of approximately $500 \mathrm{pCi} / \mathrm{L}$, which is consistent with a 
$\begin{array}{ccc}\text { Year } & \begin{array}{c}\text { Colorado River } \\ \text { Recharged (acre-ft) }\end{array} & { }^{3} \mathrm{H} \text { Colorado River } \\ \mathrm{pC} / \mathrm{L} \text { (Michel, 1992) } & { }^{3} \mathrm{H} \text { Santa Maria, CA } \\ \mathrm{pC} \text { Ci/L (IAEA, 1981) }\end{array}$

\begin{tabular}{crr}
1963 & $2.17 \mathrm{E}+05$ & 3456 \\
1964 & $1.85 \mathrm{E}+05$ & 3456 \\
1965 & $1.32 \mathrm{E}+05$ & 2592 \\
1966 & $1.16 \mathrm{E}+05$ & 2240 \\
1967 & $1.14 \mathrm{E}+05$ & 1600 \\
1968 & 91797 & 1280 \\
1969 & 52354 & 120 \\
1970 & 84938 & 960 \\
1971 & 55575 & 880 \\
1972 & 34327 & 640 \\
1973 & 52871 & 576 \\
1974 & 48292 & 512 \\
1975 & 51801 & 384 \\
1976 & 14908 & 352 \\
1977 & 15138 & 320 \\
1978 & 58660 & 304 \\
1979 & 19412 & 272 \\
1980 & 34643 & 192 \\
1981 & 33108 & 208 \\
1982 & 37575 & 176 \\
1983 & 14821 & 160 \\
1984 & 15114 & 118 \\
1985 & 32010 & 115 \\
1986 & 30235 & 110 \\
1987 & 27626 & 110 \\
1988 & 39634 & 100 \\
1989 & 2897 & 75 \\
1990 & 27395 & 50 \\
1991 & 15619 & 70 \\
1992 & 51672 & 75.976 \\
1993 & 26293 & 72.293 \\
1994 & 78521 & 68.901 \\
1995 & 14668 & 65.768 \\
1996 & 0 & 62.868 \\
& & \\
$*$ * computed by mass balance equation assuming that annual recharge was \\
\hline 19 & &
\end{tabular}
recharge and total recharge was equal to SAR recharge.

\begin{tabular}{|c|c|c|c|c|c|}
\hline \multirow[t]{2}{*}{$\underset{\mathrm{pCi} / \mathrm{L}}{* \text { Weighted }}{ }^{3} \mathrm{H}$} & \multirow[t]{2}{*}{$\underset{\mathrm{pCi}}{\text { Recharged }}{ }^{3} \mathrm{H}$} & well measured & calc. ${ }^{3} \mathrm{H}$ initial & well measured & calc. ${ }^{3} \mathrm{H}$ initial \\
\hline & & A42 1010' & 156.7 & SAR7 Z2 & 32.9 \\
\hline 3293.2 & $1.01 \mathrm{E}+15$ & A $42650^{\prime}$ & 111.4 & SAR8 Z2 & 58.6 \\
\hline 2785.8 & $8.59 \mathrm{E}+14$ & A42 $780^{\circ}$ & 133.2 & WBS2A Z2 & 21.8 \\
\hline 1656.2 & $5.10 \mathrm{E}+14$ & A42 910' & 151.7 & WBS2A Z3 & 33.4 \\
\hline 1180.2 & $3.64 \mathrm{E}+14$ & A43 625' & 72.4 & WBS3 Z2 & 20.2 \\
\hline 830.85 & $2.56 \mathrm{E}+14$ & A43 $720^{\prime}$ & 62.6 & WBS4 Z2 & 74.9 \\
\hline 569.23 & $1.75 E+14$ & A44 540' & 53.9 & WBS4 Z3 & 24.5 \\
\hline 363.57 & $1.12 \mathrm{E}+14$ & A44 670' & 84.6 & A- 47 & 40.2 \\
\hline 402.22 & $1.24 \mathrm{E}+14$ & A44 $840^{\prime}$ & 15.6 & SCWC-PLJ2 & 48.3 \\
\hline 330.01 & $1.02 \mathrm{E}+14$ & A44 980' & 247.5 & AM-7 & 24 \\
\hline 159.65 & $4.92 \mathrm{E}+13$ & AM4 & 19.3 & AM- 6 & 31 \\
\hline 174.8 & $5.39 \mathrm{E}+13$ & AM5 & 21.9 & HG-1 & 19 \\
\hline 158.28 & $4.88 \mathrm{E}+13$ & AM5A & 20 & ABS-2 & 32 \\
\hline 130.31 & $4.02 \mathrm{E}+13$ & AM11 & 21.2 & A-42 & 110 \\
\hline 69.137 & $2.13 \mathrm{E}+13$ & AM12 & 18.9 & A-43 & 67 \\
\hline 73.489 & $2.26 \mathrm{E}+13$ & AM15 & 45.2 & YLWD-5 & 64 \\
\hline 115.42 & $3.56 \mathrm{E}+13$ & AM17 & 312.7 & YLWD-11 & 27 \\
\hline 59.49 & $1.83 \mathrm{E}+13$ & AM18 & 243.8 & YLWD-15 & 28 \\
\hline 62.441 & $1.92 \mathrm{E}+13$ & AM30 & 220.3 & SID-4 & 26 \\
\hline 66.413 & $2.05 \mathrm{E}+13$ & AM33 & 51.6 & SID-3 & 33 \\
\hline 61.8 & $1.90 \mathrm{E}+13$ & AM35 & 326.8 & $0-23$ & 160 \\
\hline 42.599 & $1.31 \mathrm{E}+13$ & AMD3 Z4 & 61.9 & EOCW-E & 102 \\
\hline 31.186 & $9.61 \mathrm{E}+12$ & AMD3 Z6 & 91.2 & AMD-9/2 & 20 \\
\hline 42.627 & $1.31 \mathrm{E}+13$ & AMD3 Z9 & 251.3 & AMD-9/4 & 173 \\
\hline 35.807 & $1.10 \mathrm{E}+13$ & AMD4 Z10 & 18.3 & A- 28 & 20 \\
\hline 29.234 & $9.01 \mathrm{E}+12$ & AMD4 Z3 & 146.6 & AMD-1/4 & 20 \\
\hline 32.01 & $9.87 \mathrm{E}+12$ & AMD4 Z4 & 145 & AMD-1/7 & 30 \\
\hline 13.521 & $4.17 \mathrm{E}+12$ & AMD4 Z9 & 453.8 & AMD-1/8 & 60 \\
\hline 14.027 & $4.32 \mathrm{E}+12$ & AMD5 Z3 & 93.5 & AMD-1/9 & 50 \\
\hline 13.749 & $4.24 \mathrm{E}+12$ & AMD5 Z4 & 98.1 & AMD-2/1 & 30 \\
\hline 23.637 & $7.28 \mathrm{E}+12$ & AMD5 Z5 & 245.6 & AMD-2/4 & 43 \\
\hline 16.552 & $5.10 \mathrm{E}+12$ & AMD5 Z8 & 264.3 & AMD-2/5 & 70 \\
\hline 28.5 & $8.78 \mathrm{E}+12$ & AMD6 Z3 & 149.5 & AMD-2/7 & 256 \\
\hline 13.272 & $4.09 \mathrm{E}+12$ & AMD6 Z5 & 155.6 & AMD-2/8 & 90 \\
\hline \multirow[t]{14}{*}{10} & \multirow{14}{*}{ 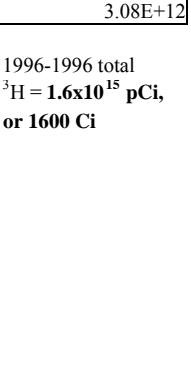 } & AMD6 Z8 & 207.1 & SAR- $6 / 2$ & 50 \\
\hline & & AMD7 Z4 & 103.3 & SAR- $6 / 4$ & 65 \\
\hline & & AMD7 Z6 & 135.2 & SAR-7/4 & 50 \\
\hline & & AMD7 Z8 & 611.4 & SAR- $7 / 5$ & 35 \\
\hline & & AMD7 Z10 & 677.8 & SAR- $7 / 6$ & 35 \\
\hline & & $\mathrm{CB} 1 \mathrm{Z} 2$ & 47.6 & AM-8 & 24 \\
\hline & & CB1 Z3 & 259.3 & AM-9 & 20 \\
\hline & & CB1 Z5 & 400.3 & AM-13 & 25 \\
\hline & & CB1 Z6 & 14.9 & AM-14 & 25 \\
\hline & & FKIM & 71 & SCWC-PBF4 & 250 \\
\hline & & F4 & 126.4 & A- -44 & 67 \\
\hline & & F8 & 164.7 & & \\
\hline & & A16 & 334.4 & & \\
\hline & & SAR6 Z1 & 33 & & \\
\hline
\end{tabular}

Oldest age measured in this set (well O-23) represents recharge in 1966. mean ${ }^{3} \mathrm{Ho}=110 \mathrm{pCi} / \mathrm{L}$ represented by recharge between 1966-1998: assume $30 \mathrm{mi}^{2}$ area and $500 \mathrm{ft}$ depth at $25 \%$ porosity

Equates to $2.96 \mathrm{e}^{12} \mathrm{~L}$, which suggests

total ${ }^{3} \mathrm{H}$ inventory of $2.96 \mathrm{e}^{12} \mathrm{X} 110=\mathbf{3 . 3 \mathrm { e } ^ { 1 4 }} \mathrm{pCi}$ or $330 \mathrm{Ci}$ 
concentration observed in 1974 (Table 6). In this case, the mixture of a groundwater, such as a 20 year old "local" recharge (initial ${ }^{3} \mathrm{H} \sim 60 \mathrm{pCi} / \mathrm{L}$ ), with Colorado River at a 90:10 percent ratio, would produce an initial ${ }^{3} \mathrm{H}$ of approximately $110 \mathrm{pCi} / \mathrm{L}$.

The probable source of local recharge to well A-42 would be from the Chino Hills or Yorba Linda sub-basin areas. This would entail infiltration from annual precipitation and migration at depth to A-42. In the case of the Chino Hills, the shortest distance from this source to A-42 ( 15,000 feet) would suggest an annual linear velocity of about $750 \mathrm{ft} / \mathrm{yr}$. This velocity is similar to that calculated for the 900-1000 foot depth in the Forebay groundwater west of the recharge basins (see below).

\section{Discussion of Groundwater Ages}

\subsection{General Uncertainties in Age Determinations}

The regular distribution of the ${ }^{3} \mathrm{H}-{ }^{3} \mathrm{He}$ ages in the Forebay groundwater illustrated in Figure 7 clearly show the direction of groundwater flow and its relative transport rate. However, before more detailed analysis of these data are performed, it is important to discuss minor limitations and uncertainties associated with these age determinations. A number of factors can influence the measured ${ }^{3} \mathrm{H}$ and ${ }^{3} \mathrm{He}$ concentration and the calculation of an age, and those related to the noble gas measurements are outlined in Appendix 1. In addition, other factors such as those related to variable ${ }^{3} \mathrm{H}$ concentration of recharging water over time and subsequent groundwater dispersion, can result in deviations of the ${ }^{3} \mathrm{H}-{ }^{3} \mathrm{He}$ age from the true groundwater age. For example, almost all the samples collected in the Forebay consisted of either Colorado River or Santa Ana River water recharge sources, and in most cases, as shown by the $\delta^{18} \mathrm{O}$ results, the two sources are commonly mixed together in a single sample (Fig. 27). A smaller amount of State Water Project water was also recharged since the early 1970s, but its occurrence and contribution to groundwater mixtures could not be observed using $\delta^{18} \mathrm{O}$ measurements. The Colorado River and the SAR represent two distinct ${ }^{3} \mathrm{H}$ input functions controlling the ${ }^{3} \mathrm{H}-{ }^{3} \mathrm{He}$ age relation. Where these two sources mix, the measured ${ }^{3} \mathrm{H}$ and $\delta^{18} \mathrm{O}$ values are a product of linear mixing, whereas the ${ }^{3} \mathrm{He}$ from these two sources resulted from nonlinear mixing. The effects of non-linear mixing (i.e. dispersion) on calculated ages have been discussed in detail elsewhere for a single recharge source with one ${ }^{3} \mathrm{H}$ fallout record (e.g. Schlosser et al., 1989; Solomon and Sudicky, 1991; Stute et al., 1997).

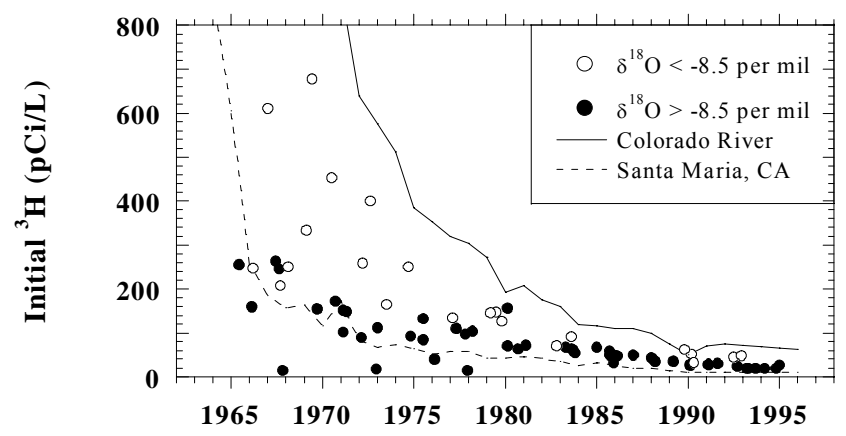

Figure 27. Most groundwater initial tritium concentrations follow the Santa Maria tritium fallout, but some depart because of mixtures from Colorado River water, which had higher tritium fallout. The highest initial tritium concentrations in groundwater correlated to the lowest $\delta^{18} \mathrm{O}$ value, consistent with Colorado River mixtures. 
Decay of ${ }^{3} \mathrm{H}$ to ${ }^{3} \mathrm{He}$ from these two different fallout concentrations and subsequent mixing would result in weighting of the mixed sample towards the higher initial ${ }^{3} \mathrm{H}$ source. Therefore, in the case where a young groundwater $(<10$ years old $)$ derived from the SAR mixes with an older Colorado River water $(>20$ years old), the non-linear mixing causes a weighting towards the older Colorado River age. Given this non-linear condition, the ${ }^{3} \mathrm{H}_{-}^{3} \mathrm{He}$ ages should be treated as weighted mean ages. For a highly dispersive flow system, as is the condition for the Forebay, a groundwater sample likely comprises a mixture of ages distributed over a range greater than the weighted mean. In general, for weighted mean ages of groundwater recharged after the ${ }^{3} \mathrm{H}$ fallout peak, the ${ }^{3} \mathrm{H}-{ }^{3} \mathrm{He}$ age is older than the true age, whereas, for groundwater recharged before the ${ }^{3} \mathrm{H}$ fallout peak, the ${ }^{3} \mathrm{H}-{ }^{3} \mathrm{He}$ age tends to be younger than the true age (e.g. Solomon and Sudicky, 1991).

The ${ }^{3} \mathrm{H}$ inventory in the Forebay groundwater contributed by each recharge source from 1963 through 1996 was calculated by weighting ${ }^{3} \mathrm{H}$ fallout concentrations with the annual recharge records (Table 6). A comparison of the historical ${ }^{3} \mathrm{H}$ fallout concentrations between the Santa Maria precipitation and the Colorado River water indicates that the much higher ${ }^{3} \mathrm{H}$ concentration of the latter would contribute the majority of ${ }^{3} \mathrm{H}$ in any groundwater mixture in the Forebay. The results suggest that the Colorado River water contributed around five times more ${ }^{3} \mathrm{H}$ to recharge in the Forebay than did the SAR for this time period.

It is useful to calculate a ${ }^{3} \mathrm{H}$ inventory of the Forebay groundwater using the initial ${ }^{3} \mathrm{H}$ values derived from the ${ }^{3} \mathrm{H}-{ }^{3} \mathrm{He}$ age determinations. This ${ }^{3} \mathrm{H}$ inventory can then be compared to the calculated inventory estimated from ${ }^{3} \mathrm{H}$ fallout, surface water records, and recharge history. The study area, defined by observations of the ${ }^{3} \mathrm{H}$ and ${ }^{3} \mathrm{He}$, encompasses approximately $30 \mathrm{mi}^{2}$ and has an average depth of about 550 feet. Assuming an average porosity of $25 \%$, a total instantaneous water volume of $1.1 \times 10^{11} \mathrm{ft}^{3}$, or $3.3 \times 10^{12}$ liters is calculated. The range in ${ }^{3} \mathrm{H}-{ }^{3} \mathrm{He}$ ages suggest that groundwater in the aquifers was recharged between 1966 and 1997. An arithmetic mean initial ${ }^{3} \mathrm{H}$ concentration derived from all these measurements is $110 \mathrm{pCi} / \mathrm{L}$. Consequently, multiplying the instantaneous water volume with this mean initial ${ }^{3} \mathrm{H}$ value suggests that approximately $3.6 \times 10^{14} \mathrm{pCi}$, or $360 \mathrm{Ci}$ of ${ }^{3} \mathrm{H}$ were recharged to the Forebay over the suggested 31 year recharge history reflected in the data. From the data in Table 6, an estimate of ${ }^{3} \mathrm{H}$ fallout can also be calculated for 1966-1997. Integrating this amount of ${ }^{3} \mathrm{H}$ recharged to the Forebay weighted to each recharge source, suggest that $1.4 \times 10^{15} \mathrm{pCi}$, or $1400 \mathrm{Ci}$ of ${ }^{3} \mathrm{H}$ was potentially recharged to the groundwater system based on the ${ }^{3} \mathrm{H}$ fallout records. This calculated amount of ${ }^{3} \mathrm{H}$ is around four times higher than estimated from the initial ${ }^{3} \mathrm{H}$ inventory calculated from the ${ }^{3} \mathrm{H}-{ }^{3} \mathrm{He}$ ages. The Colorado River contributed the greater amount of ${ }^{3} \mathrm{H}$ during over the recharge history, although it is likely the fallout records from this source are overestimated. In particular, the fallout was measured in Utah, whereas Colorado River water recharged in the Forebay transcended over a 1000 miles and was stored in surface water reservoirs for weeks to months. This likely contributed to ${ }^{3} \mathrm{H}$ loss due to atmospheric exchange.

It is worth reviewing the possibility that tritiogenic ${ }^{3} \mathrm{He}$ was lost or diffused out of groundwater after its accumulation from ${ }^{3} \mathrm{H}$ decay (Schlosser et al., 1989). In this case, the loss of tritiogenic ${ }^{3} \mathrm{He}$ through diffusion out of the aquifer toward the water table would have to occur at a rate that exceeds the rate at which it can by advective transport 
vertically and laterally downgradient in the aquifer. The tritiogenic ${ }^{3} \mathrm{He}$ loss is mostly dependent on the vertical velocity and the dispersion coefficient (Schlosser et al., 1989). In addition, thick unsaturated zones would also lead to diffusive loss of tritiogenic ${ }^{3} \mathrm{He}$. For simple aquifer models using steady-state isotropic conditions, it has been shown that tritiogenic ${ }^{3} \mathrm{He}$ loss was insignificant for vertical velocity $>1.5 \mathrm{ft} / \mathrm{yr}$ and a dispersion coefficient $<2.7 \mathrm{ft}^{2} / \mathrm{yr}$ (Schlosser et al., 1989). The greater the dispersion coefficient and the lower the vertical velocity, the greater the tritiogenic ${ }^{3} \mathrm{He}$ loss.

For the Forebay aquifer system, vertical velocities of recharge originating from the percolation basins are significantly greater than $1.5 \mathrm{ft} / \mathrm{yr}$, and even though dispersion may be high due to aquifer heterogeneity, the loss of tritiogenic ${ }^{3} \mathrm{He}$ by diffusion to the water table is unlikely. Thick unsaturated zones do not conduct recharge in the Forebay. Even during basin infiltration following basin cleaning recharge does not necessarily occur in unsaturated conditions, but rather likely as discrete saturated flow paths, as suggested by the xenon tracer results.

For SAR channel recharge, vertical velocity may have a greater range because it is likely that the fastest flowpaths from the river channel are along the water table, and that discontinuous layering of lower permeability sediments may limit some vertical transport. However, given that annual recharge from the river channel exceeds 70,000 acre-ft, and that the geographic extent of annual recharge from river channel is $<10 \mathrm{mi}^{2}$, the vertical transport rate on average must significantly exceed $1.5 \mathrm{ft} / \mathrm{yr}$, and is probably closer to $10 \mathrm{ft} / \mathrm{yr}$. It is therefore concluded that loss of tritiogenic ${ }^{3} \mathrm{He}$ during recharge and flow is not a significant process contributing to the ${ }^{3} \mathrm{H}-{ }^{3} \mathrm{He}$ age determination. Uncertainties in the age calculation simply result from accumulative addition of each analytical measurement and each calculation used to derive the age. Uncertainties are greatest where ages are young (low tritiogenic ${ }^{3} \mathrm{He}$ ) and excess air corrections are high. In addition, where a radiogenic ${ }^{4} \mathrm{He}$ term was subtracted, the uncertainty increased.

\subsection{Hydrologic Implications of Age Determinations}

The ${ }^{3} \mathrm{H}-{ }^{3} \mathrm{He}$ age distribution in Figure 7 illustrates groundwater flow directions and relative transport rates. However, this age contour map was generated by comparing ages from wells ranging over several hundred feet in depth and by averaging ages in multipoint monitoring wells. In order to better represent the age distribution in the Forebay groundwater, age contour maps were generated for three different depth ranges represented by the measured wells (Figs. 28a-c). The second layer (Fig. 28b) has the most ${ }^{3} \mathrm{H}-{ }^{3} \mathrm{He}$ age determinations available for contouring, however, several points can be illustrated for all three layers. For example, the ages in the upper layer (200-300 ft below the surface; see Fig. 28a) indicate that recharge of $<1$ year old transcends a distance of greater than one mile from the recharge basins, following a southwesterly path, and implies a linear groundwater velocity $>5000 \mathrm{ft} / \mathrm{yr}$. The five year old groundwater contour in Figure 28a extends west to nearly 2.5 miles from the recharge basins. In contrast, shallow groundwater beneath the SAR channel ranges from 1 to 10 years old, occurring in isolated pockets, and suggests that recharge from the SAR does not occur by deep vertical penetration as seen below the basins. 

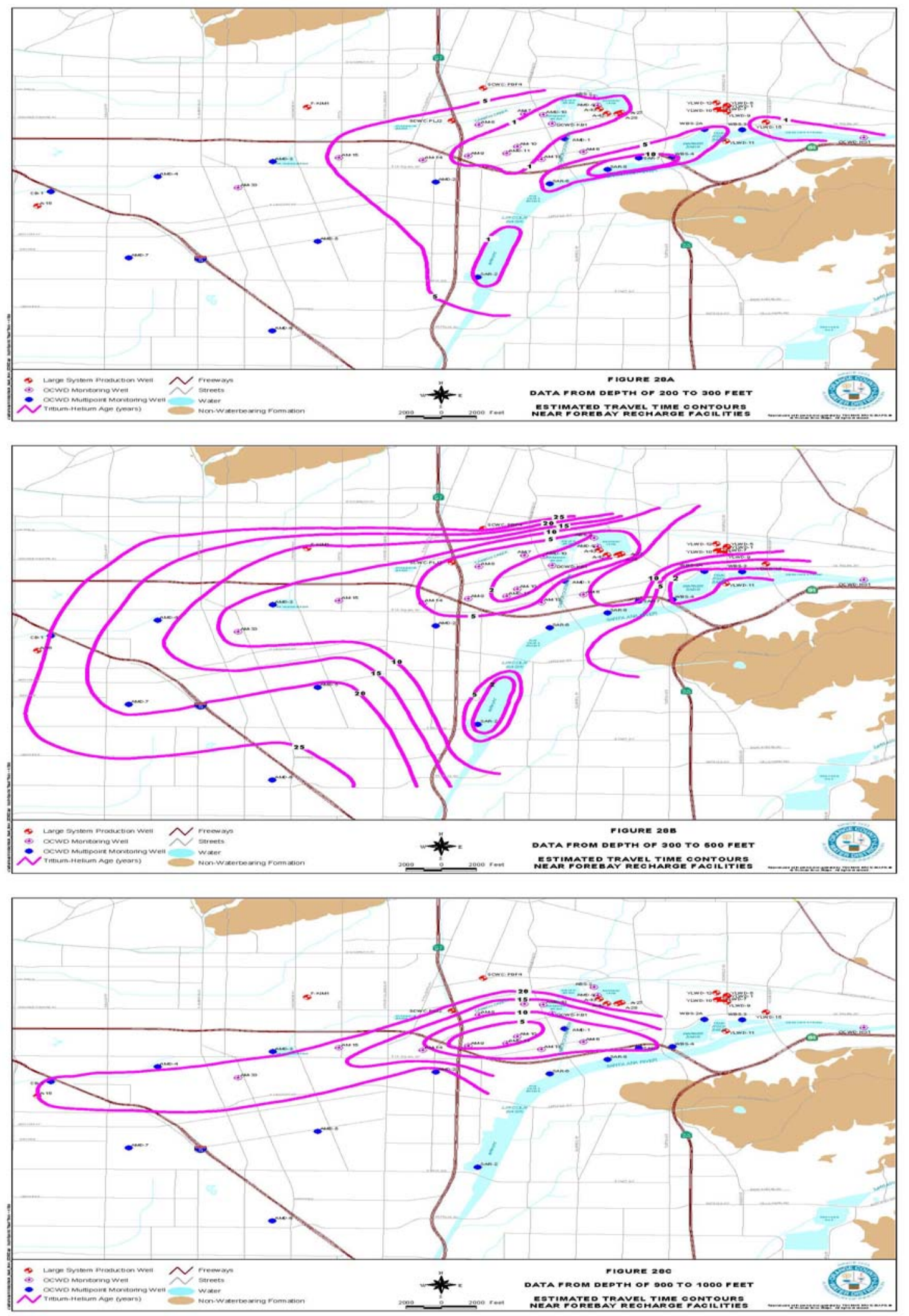

Figure 28. The ${ }^{3} \mathrm{H}-{ }^{3} \mathrm{He}$ ages were contoured for three different depth intervals: a) 200-300 feet, b) 300-500 feet, and c) 900-1000 feet below the surface. The 300-500 feet interval has the most data and illustrates the rapid infiltration and westward transport of basin recharge at this depth. 
For the 300-500 feet below surface contours (Fig. 28b), the ${ }^{3} \mathrm{H}-{ }^{3} \mathrm{He}$ ages are somewhat older than in the shallower depth at the same geographic location. The youngest contour of two years old surrounds the recharge basins. The five year contour west of the recharge basins has a similar alignment as in the shallower depth, and implies a mean linear groundwater velocity of around $2000 \mathrm{ft} / \mathrm{yr}$. The more extensive data coverage of this layer illustrates the regular distribution of the 10 through 25 year old age contours west of the recharge basins. The distance to the 10 year contour is greater from Anaheim Lake and Kraemer Basin compared to the distance to the SAR, suggesting relatively slower linear velocities for recharge moving perpendicular away from the SAR channel. Also, note the spacing among the 10 to 25 year old age contours is much closer than for the younger age contours, suggesting linear velocity decreases west from the recharge basins. This is expected since the groundwater basin increases width and depth away from the recharge basins. Also note that the ages increase sharply just to the north of Anaheim Lake and Miller Basin, consistent with older groundwater in the Yorba Linda sub-basin flowing southwestward into the Forebay. The 10 year age contour drawn between Anaheim Lake and Warner Basin was drawn to suggest local recharge from the Chino Hills, but data control is lacking.

The deepest layer is from 900 to 1000 feet below the surface (Fig. 28c). Data are limited to wells west and southwest of Anaheim Lake and Kraemer Basin. The age contours suggest young groundwater less than five years old penetrates deep into the aquifer approximately one mile southwest of the Anaheim Lake and follows a flow path to the west into the Forebay. The age contours imply mean linear groundwater velocity at this depth between 1000 and $1500 \mathrm{ft} / \mathrm{yr}$.

In cross section the ${ }^{3} \mathrm{H}-{ }^{3} \mathrm{He}$ age contours show a regular concentric pattern beneath Anaheim Lake and Kraemer Basin (Fig. 29a). Ages less than one year penetrate more than 500 feet below the surface in the area of AMD-10. The ages abruptly exceed 20 years old at $<1000$ feet below the surface where a laterally extensive low permeability layer has been recognized in several well logs in the area. Ages progressively increase to over 20 years old approximately 2-5 miles west of the recharge basins, depending on depth. The cross section of ages illustrates that beneath the basins discontinuous aquifer layering allows deep vertical recharge of basin water, while west of the basins, aquifer layering is more developed and potentially limits vertical transport. 

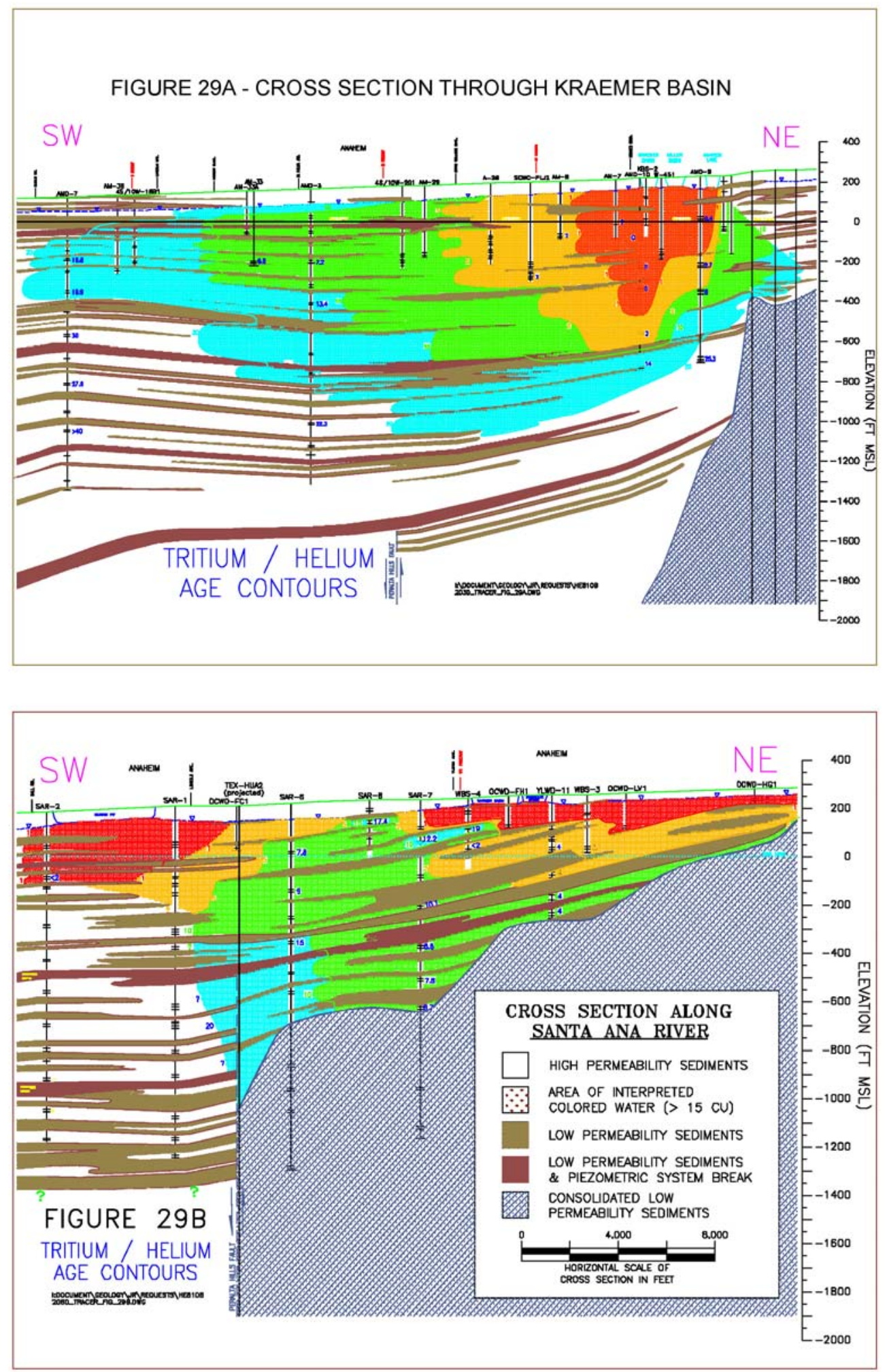

Figure 29. Cross-sections with ${ }^{3} \mathrm{H}-{ }^{3} \mathrm{He}$ age contours show a regular increasing age with depth and downgradient transport below a) Kramer Basin and Anaheim Lake, whereas, b) beneath the SAR, age contours are more irregular, suggesting retarded transport at some locations, particularly near the Peralta Hills fault. 
The ${ }^{3} \mathrm{H}-{ }^{3} \mathrm{He}$ contours beneath the SAR channel illustrate a recharge process different from what occurs below the recharge basins (Fig. 29b). For example, ages of less than one year are observed in relatively shallow depths between HG-1 and SAR-7, and below Burris Pit. Ages greater than 10 years old also occur in shallow levels between SAR-7 and SAR-1 and suggest an area where recharge is limited. These older ages may also suggest a zone where deeper, older groundwater is focussed upward because of changes in basement bathymetry (Fig. 29b). In addition, the occurrence of the Peralta Hills fault just west of SAR-6 may retard westward motion of groundwater at depth and cause upward flow of older groundwater. The large volume of annual recharge percolated from the SAR channel $(>70,000$ acre-ft per year) likely occurs by only shallow vertical penetration below the channel ( $<100$ feet). Extensive lateral and river-parallel transport, dominated by flow paths near the water table, likely moves most of the recharged river water away from the SAR into the Forebay.

\section{Discussion of Flowpaths and Flow Rates}

11.1 Co-mingling of Different Recharge Sources in Forebay Groundwater

One feature worth illustrating using the ${ }^{3} \mathrm{H}-{ }^{3} \mathrm{He}$ ages and the $\delta^{18} \mathrm{O}$ values is the location of Colorado River recharge over a period from 1966 to 1992. Over this time period, Colorado River water was only recharged in Anaheim Lake, Miller Basin, and Kraemer Basin. This is best shown in the depth interval of 300 to 500 feet below the surface where the highest ${ }^{3} \mathrm{H}-{ }^{3} \mathrm{He}$ age data density was contoured (Fig. 30). In general, an area can be circumscribed to represent groundwater with a mixture of Colorado River water as determined by its $\delta^{18} \mathrm{O}$ value. This area forms an ellipsoidal shape stretching northeastsouthwest across the western Forebay. AMD-7 is the farthest downgradient well where Colorado River water was observed, however, wells farther downgradient also should have Colorado River mixtures. The absence of Colorado River water in AMD-2, AMD-5 and AMD-6 defines a southward limit to Anaheim Lake and Kraemer Basin recharge at the 300-500 foot depth interval. Because Colorado River recharge was observed in well AM-10 in 1995, the southernmost flow path from these basins likely follows a line approximately through AM-10 flowing west and north of AMD-5. The exact boundary of southerly flow from the basins varies over time because of variable head differences between river recharge and basin recharge, but in general the distribution of Colorado River water in figure 30 provides a delineation where preferential recharge from Anaheim Lake, Kraemer Basin, and Miller Basin occurs on average over time. The mixture of Colorado River water and SAR sources in the Forebay is likely due to pumping from multiple perforation levels in production wells and dispersive mixing along flow paths as the two recharge sources are alternately percolated in the basins. 


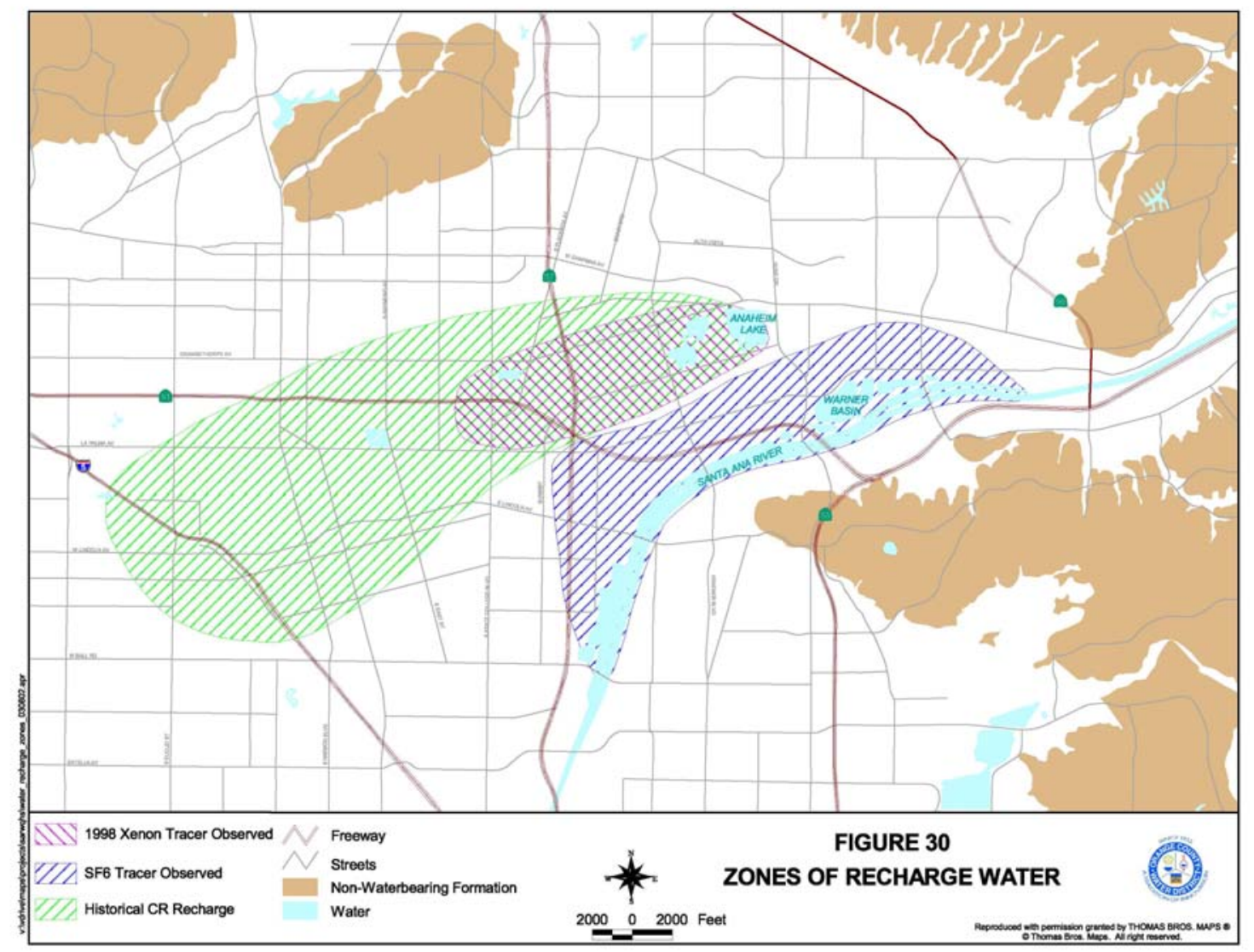

Figure 30. Groundwater that had mixtures of Colorado River water were geographically delineated and indicate areas under the influence of Anaheim lake and Kramer Basin recharge. Likewise, the geographic demarcation of $\mathrm{SF}_{6}$ and $\mathrm{Xe}$ isotope tracers indicate limited lateral mixing and segregation of SAR and basin recharge downgradient.

The distinction of SAR channel recharge from basin recharge was further facilitated by the simultaneous tracer experiments using isotopically enriched xenon and $\mathrm{SF}_{6}$. Recall that the xenon tracer was introduced into both Anaheim Lake and Kraemer Basin in October 1998, while the $\mathrm{SF}_{6}$ was released to the SAR channel in July 1998. The observation and geographic extent of these tracers after two years of measurement are also illustrated in figure 30. Note that $\mathrm{SF}_{6}$ and a xenon tracer were never observed simultaneously or at different times in any one well. Lack of co-mingling of these two tracers suggests that recharge from the SAR and the recharge basins follow different flow paths. This is not to suggest that permeable layers beneath the recharge basins and the SAR channel are not interconnected, which undoubtedly they are, given the continuity of permeable material in the eastern Forebay. More than likely the lack of co-mingling is due to low lateral dispersion during groundwater transit, and rapid longitudinal advection in a westward direction. Both the SAR channel and the recharge basins, when full, maintain hydrologic head on the aquifer system and the distinct flow paths create opposing forces against each other that limits dispersive interaction. The propensity for lateral co-mingling between flow paths originating from these two sources is likely controlled primarily by diffusive processes, which transport mass less effectively than 
advective/dispersive processes. However, once the hydrologic head is significantly decreased in either recharge source (e.g. basin cleaning), the condition likely arises that allows encroachment of groundwater flow by lateral dispersion into permeable layers connected to the other recharge source.

\subsection{Tracer Travel Times}

The accumulation of tracer breakthrough data from the $\delta^{18} \mathrm{O}$, xenon isotopes, and the sulfur-hexafluoride experiments provides a near-comprehensive picture of groundwater recharge, transport paths, and transit times to wells recharged by the SAR, Anaheim Lake, and Kraemer Basin (Table 7). Linear distances were estimated between the downstream side of Anaheim Lake and Kraemer Basin to specific wells where Colorado River water and xenon was observed. Distances from specific wells to their SAR recharge points were estimated from map view also, but took into account the direction of groundwater flow using water table contours. This was the same approach used by Gamlin et al. (2001) for their assessment of travel times, and good agreement resulted (slope $=1$; largest difference was $500 \mathrm{~m}$ ) between these independent determinations.

In some situations it is more important to know the fastest flow rate. For example, in the case of microbial transport to a drinking water source, where potential exposure may present acute health risks independent of dose, the earliest arrival time to a particular well would be the most critical parameter. Therefore, it is important to characterize both the fastest flow rates and the mean flow rates for full consideration of transport and fate of potential contaminants. Arrival times for the tracer data are designated as first appearance of the tracer in any given well. This represents the minimum transport time from the recharge source to the well.

Table 7 indicates arrival times (fast flow paths) at the monitoring and production wells vary from less than one month to greater than two years. Plotting the distances to wells versus the arrival times provides an indication of the range in maximum groundwater velocity for the Forebay (Fig. 31). The data generally form a bimodal distribution, with most lying along a linear trend with a slope of approximately 8800 feet per year. These data were derived using the $\delta^{18} \mathrm{O}$, xenon and sulfur-hexafluoride tracer results. Similarities of velocity among these different sites suggest aquifer materials can be represented by similar bulk transport parameters throughout the shallow groundwater aquifers of the Forebay. Furthermore, the linear trend suggests that groundwater on average flows at a maximum rate of around 8800 feet per year. Using a water table gradient of 0.01 and a mean porosity of $25 \%$, a hydraulic conductivity of $\sim 600 \mathrm{ft} / \mathrm{day}$ is derived. This value is within the range for coarse sand and gravel material (Dominico and Schwartz, 1990) 


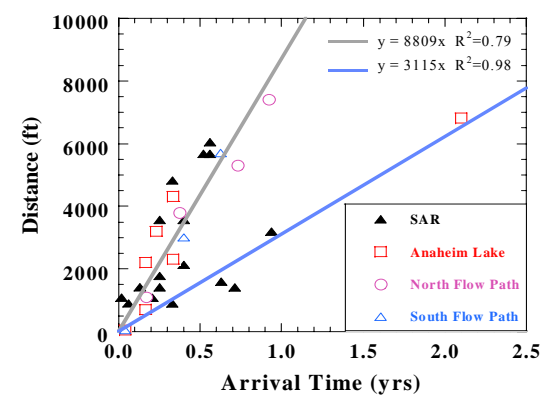

Figure 31. Based on arrival times of tracers, the groundwater velocity in the recharge areas of the Forebay are relatively uniform, averaging $>8000$ $\mathrm{ft} /$ year for fastest flow, except for groundwater flow presumably retarded by the Peralta Hills fault (lower slope).

A few points in figure 31 form a line with a lower slope $(3115 \mathrm{ft} / \mathrm{yr})$. These are wells AMD-11 level 2, AM-11, AM-46, and SAR-6/1. In these cases the arrival times are much longer relative to their distances from recharge points. These well locations lie along a line that extends northwest from the SAR above Burris pit and parallel to Peralta Hills fault trace (see Fig. 2d). This fault could impede groundwater flow and produce the lower velocities observed for these four wells.

The arrival time for wells receiving tracer in the Forebay can be contoured to better understand where preferred recharge and flow occur (Fig. 32). Arrival times show a consistent and regular increase with distance down gradient of Anaheim Lake and Kraemer Basin. This contrasts the distribution of arrival times adjacent to SAR recharge. In particular, arrival times appear to be fastest adjacent to the SAR west of Warner Basin, but east of well SAR-6. Groundwater contours are nearly parallel to the SAR in this region and a northward flow direction is implied, consistent with water table levels. Arrival times are much slower west of this area defined by the low velocity zone near the Peralta Hills fault. The impedance of flow in the area of these wells appears to force SAR recharge to flow northward and around this low velocity zone. Fast recharge is also suggested by fast arrival times along the SAR in the vicinity of Warner Basin and further east. 
Table 7. Transport Rates Derived from $\mathrm{SF}_{6}$ and Xenon isotope data.

well

distance from first arrival recharge source $(\mathrm{ft})$ (days)

AM-7

AM-8

SCWC-PLJ2

A-26

OCWD-KB1

AM-10

AM-9

AM-14

A-27

AM-44

AMD-10/1

AMD $-10 / 2$

AMD-10/3

AMD-10/4

OCWD-LV1

WBS2A/2

2112
4750
6000
8000
500
3875
6375
8875
500
1500
4000
4000
4000
4000
1800
2525

2112

4750

8000

500

3875

6375

500

1500

4000

4000

4000

1800

2525 center of mass last arrival arrival (days) (days)

57
125
224
335
19
105
185
322
13
62
-
-
-
-
77
91

max velocity mean velocity min. velocity $\mathrm{ft} / \mathrm{d}$

$\begin{array}{rrrr}167 & 37 & 19.7 & 13 \\ 382 & 38 & 16.9 & 12 \\ 619 & 27 & 16.6 & 10 \\ 800 & 34 & 16.6 & 10 \\ 38 & 26 & 19.2 & 13 \\ 378 & 37 & 16.8 & 10 \\ 512 & 34 & 18.2 & 12 \\ 800 & 28 & 17.3 & 11 \\ 67 & 38.5 & 18.5 & 7.5 \\ 138 & 24 & 16.5 & 11 \\ - & - & 27 & - \\ - & - & 12 & - \\ - & - & 9.4 & - \\ - & - & 6.5 & - \\ - & 23.3 & 10.3 & - \\ - & 27.7 & 17.5 & -\end{array}$




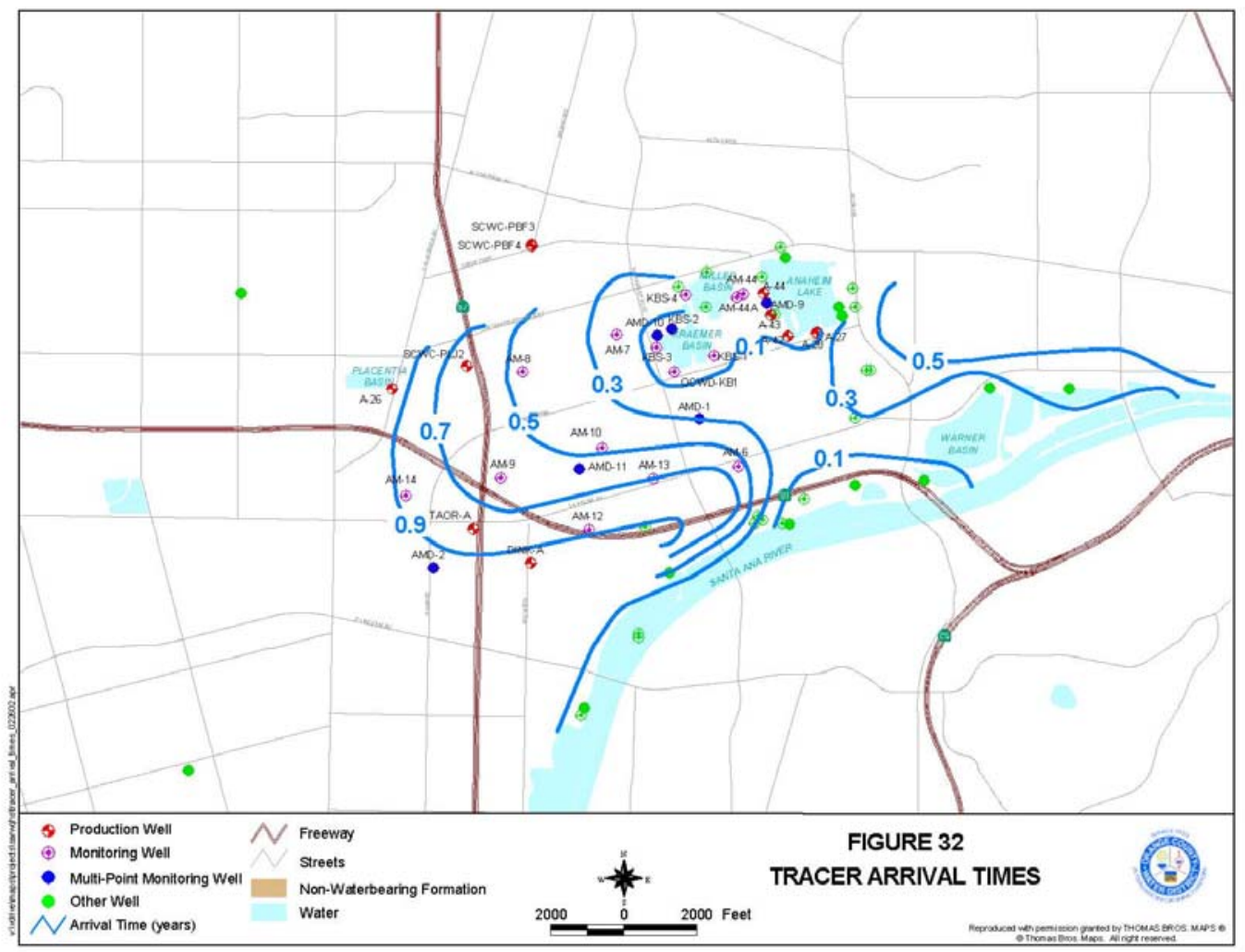

Figure 32. Contours of first arrival time (in years) of tracer in wells the Forebay reveal a relatively uniform velocity field for the fastest groundwater flow. Only the Peralta Hills fault area suggests retardation and heterogeneity in velocity.

From the breakthrough curves of several wells, the mean groundwater velocity can be calculated. In these cases, the tracer data forms a pulse concentration over time with an ascending and descending distribution. The center of mass of this tracer pulse approximates the mean transport time of the labeled groundwater. Center of mass can be calculated for most wells monitored downgradient of Kraemer and Anaheim Lake, but only in a few cases for those wells downgradient of the SAR (WBS-4 level 1, SAR-8 level 1, OCWD-LV1, WBS2a level 2, WBS-4 level 3, and AM-5) did the $\mathrm{SF}_{6}$ define breakthrough curves. Center of mass was calculated by integrating the total area below the tracer curve for each well, and estimating the day in which half the total area occurred. From this time estimate, a simple linear velocity was calculated (Table 7).

Consistent with figure 31, the mean linear velocities calculated for wells down gradient of Kraemer Basin have a narrow range between 16.6 to $19.7 \mathrm{ft} /$ day $(6060-7190$ $\mathrm{ft} / \mathrm{yr}$; Table 7). The two highest velocities are from wells AM-7 and OCWD-KB1, which have the closest proximity to Kraemer Basin. Center of mass estimates may have poorer precision for tracer breakthrough curves that have higher concentration, particularly if sampling was infrequent. Nevertheless, the narrow range of linear velocities suggest uniformity of aquifer parameters along the fast flow path downgradient of Kraemer Basin. Similarly, mean linear velocities calculated for A-27 and AM-44 from the 1998 xenon tracer data in Anaheim Lake also fall in the same range as those calculated for wells downgradient of Kraemer Basin. 
Calculating an arithmetic mean linear velocity from all the xenon tracer breakthrough analysis results in $17.6 \mathrm{ft} / \mathrm{day}$. Using a hydraulic gradient of 0.01 and average porosity of $25 \%$, an average hydraulic conductivity of $440 \mathrm{ft} /$ day is calculated. This value is representative of conductivity range measured in coarse sand and gravel (Dominico and Schwartz, 1990).

For well AM-44, it is interesting to compare the mean linear velocity calculated from the $1998{ }^{129} \mathrm{Xe}$ tracer data to that calculated from the $1996{ }^{124} \mathrm{Xe}$ tracer data. In particular, where in 1998, the mean velocity was $16.5 \mathrm{ft} /$ day, in 1996 it was only $12.5 \mathrm{ft} /$ day. Although this difference is not tremendous, it does suggest that the hydraulic gradient was different for these two experiments at least in the vicinity of AM-44. The most obvious difference between the two experiments was that in 1996 Miller Basin was filled with water, while in 1998 Miller Basin was left empty. The presence of water in Miller basin in 1996 could have reduced the hydraulic gradient between Anaheim Lake and AM-44 and subsequently produced a slower velocity.

The mean linear velocities calculated from the ${ }^{129} \mathrm{Xe}$ tracer data for well AMD-10 showed a systematic decrease with depth, ranging from $27.0 \mathrm{ft} /$ day for level $1,12.0 \mathrm{ft} / \mathrm{day}$ for level 2, $9.4 \mathrm{ft} /$ day for level 3, and $6.5 \mathrm{ft} /$ day for level 4 . The decreasing age with depth approximates an exponential curve fit, which is consistent with vertical profiles of ages for simulated groundwater flow in isotropic porous media (e.g. Goode, 1996). This suggests that recharge from Anaheim Lake to depths up to 750 feet below the surface west of Anaheim Lake occurs in near-uniform porous sediments, and zones of low permeability are infrequent and discontinuous.

For wells recharged by the SAR and that defined breakthrough curves during the $\mathrm{SF}_{6}$ tracer experiment, calculated mean linear velocities showed a larger range from 6.0 to $53.6 \mathrm{ft} /$ day. The arithmetic mean of all these values was approximately $21 \mathrm{ft} /$ day. Some of this variability may be attributable to error in determining the exact point of recharge, or the center of mass of the breakthrough curve. Nevertheless, the average of the mean linear velocity is still similar to that calculated for downgradient of Kraemer Basin, and representative of coarse sand to gravel aquifer material, assuming the same porosity and hydraulic gradient. The larger variability among linear velocities calculated from the $\mathrm{SF}_{6}$ data is consistent with discontinuous layering of coarse and fine sedimentary material observed near the SAR. This layering apparently forms discrete horizontal flow paths that likely transport SAR water rapidly downgradient, as also indicated by the irregular distribution of ${ }^{3} \mathrm{H}-{ }^{3} \mathrm{He}$ ages below the SAR.

One final comparison of interest is the mean linear velocities to the ${ }^{3} \mathrm{H}-{ }^{3} \mathrm{He}$ ages. This comparison is most relevant to wells along the "north" flow path originating from Kraemer Basin (Fig. 18). For these wells, ${ }^{3} \mathrm{H}-{ }^{3} \mathrm{He}$ ages were between 2 and 3 years old. Well SCWC-PLJ2 consistently showed measurable tritiogenic ${ }^{3} \mathrm{He}$, and ages calculated from three separate samples were between 2 and 3 years. However, the center of mass calculated from the xenon tracer breakthrough curve suggested a mean linear travel time of approximately one year. The difference between the ${ }^{3} \mathrm{H}-{ }^{3} \mathrm{He}$ age and the tracer age simply illustrates the difference between a mean age calculated from ${ }^{3} \mathrm{H}-{ }^{3} \mathrm{He}$ measurements and that derived from center of mass determination of a tracer. Mixing of older and younger water tends to bias the ${ }^{3} \mathrm{H}-{ }^{3} \mathrm{He}$ age toward the older aged water.

The discrepancy between ${ }^{3} \mathrm{H}-{ }^{3} \mathrm{He}$ ages and tracer ages for wells AM-7 and AM-8 are larger. For example, the tracer travel time was approximately 4 months for well AM-7, 
yet the ${ }^{3} \mathrm{H}-{ }^{3} \mathrm{He}$ age was approximately 2.5 years on two separate samples and analyses. The same age was measured on AM-8 while the tracer travel time was only about 9 months. In contrast, ${ }^{3} \mathrm{H}-{ }^{3} \mathrm{He}$ ages in the "south" flow path were less than one year for wells AM-10 and AM-9, which is consistent with tracer travel times. It is probable that a mixture of older groundwater with younger water occurs for wells along the "north" flow path. This older water may be a "local" recharge source derived upgradient to the east or north of Kraemer Basin or Anaheim Lake. This older water component could be up to 30 years old and mixes at small percentages in the "north" flow path wells. In the case of small mixtures of old water, Its influence on the tracer concentration may be insignificant, yet would perturb the ${ }^{3} \mathrm{H}-{ }^{3} \mathrm{He}$ age because of its high tritogenic ${ }^{3} \mathrm{He}$ content.

\section{Groundwater Dispersion and Dilution}

The tracer breakthrough curves observed for the various wells within the Forebay reflect a range in velocities for specific groundwater flow paths. In any particular monitoring point, the first observed tracer at the initial point of the breakthrough curve represents the "fastest" flow line (least tortuous) leading to this well, whereas the last tracer observation at the tail-end of the breakthrough curve in general is the "slowest" flow line (most tortuous). Consequently, the center of mass for the entire breakthrough curve represents the mean flow rate of the aquifer layer transporting the tracer. For derivation of physical aquifer properties such as hydraulic conductivity, it is best to use the mean flow rate. By characterizing the spread of the tracer plume, aquifer dispersion parameters can potentially be derived by analyzing changes in the tracer breakthrough over the travel distance.

Transport and movement of a dissolved substance in water can be simply described in terms of an advective process combined with a dispersive (or diffusive) term, where with conservation of mass, the solute plume migrates in a defined velocity field and the solute concentration decreases over space and time. This process is best illustrated with the xenon isotope tracer data characterized in wells associated with downgradient flow from Kraemer basin recharge. For example, comparison of wells AM-7, AM-8, SCWC-PLJ2, and A-26 that form the "north" flow path show systematic plume migration and decrease in tracer concentration with distance (Fig. 33a). Likewise wells OCWD-KB1, AM-10, AM-9, and AM-14 forming the "south" flow path show a similar tracer plume migration (Fig. 33b). A smoothing function was used to extrapolate between individual tracer points to better illustrate the change in plume shape with travel distance. In general, plumes are sharp and of short duration near the recharge basin, but quickly spread out and become elongated with distance downgradient. 

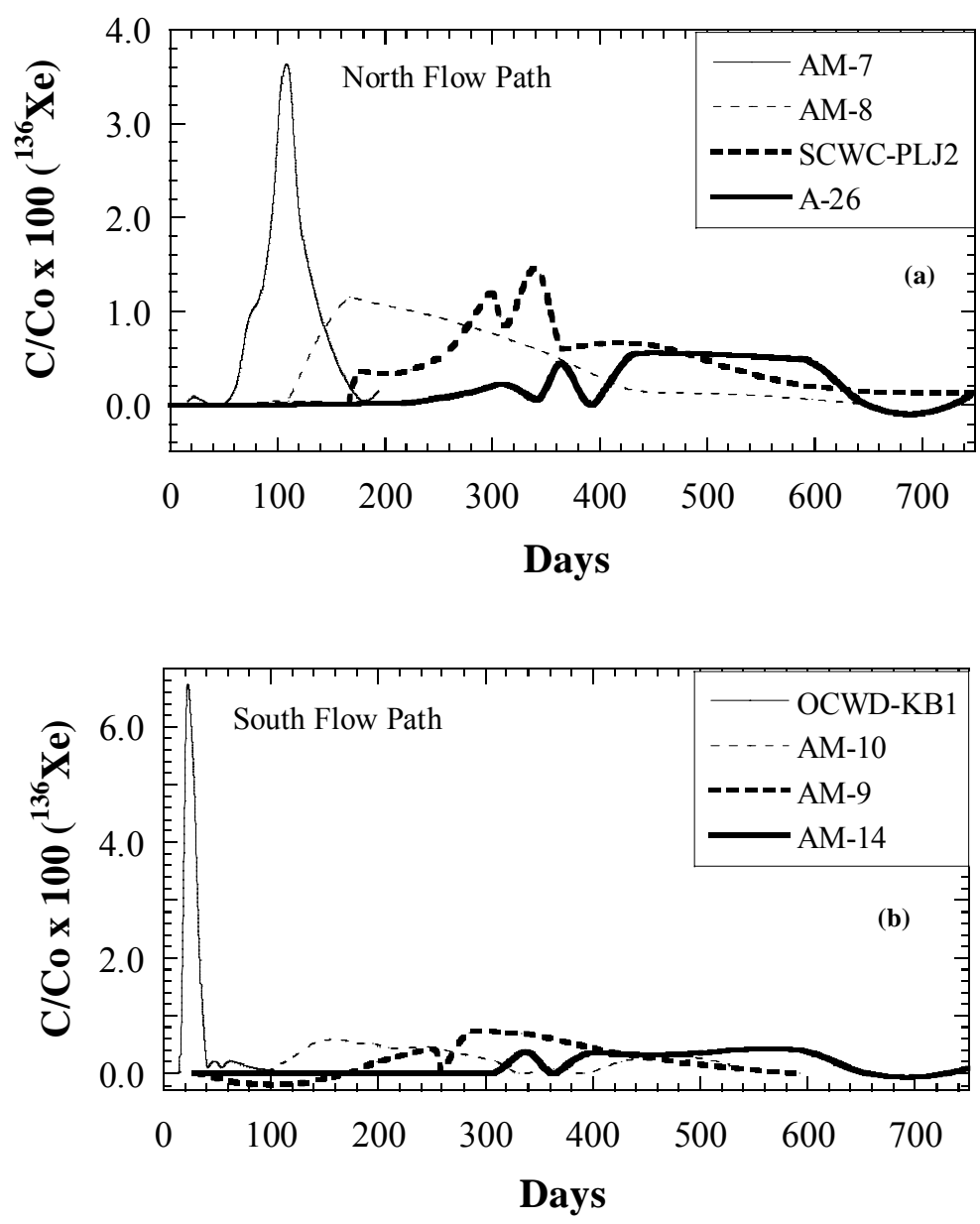

Figure 33. Smooth curve fits to the ${ }^{136} \mathrm{Xe}$ data in wells of the a) "north" flow path and b) "south" flow path illustrate the decreasing peak breakthrough concentration and widening of the curve with time and distance downgradient.

Two issues of importance regarding the tracer migration are the 1) conservation of tracer mass, and 2) the rate of dispersion of the tracer plume. Conservation of mass is assumed in tracer transport through the Forebay system, however, its quantification is not straight forward and lends itself to possible confusion. For example, it is tempting to simply treat the tracer data in a one-dimensional flow field and integrate the area under the tracer curves. However, in doing so, it is assumed that lateral and vertical migration of the tracer is insignificant compared to longitudinal transport. Furthermore, onedimensional treatment of the data ignores the potential for perforated depths in monitoring wells to capture more than one flow path transporting the tracer. In this case, the tracer concentration could be persistent, and integration over time results in excess tracer mass. As an example, integrating the ${ }^{136} \mathrm{Xe}$ tracer concentration modeled for Kraemer Basin (Fig. 16c; adding daily modeled concentrations over the entire tracer duration), results in an apparent starting ${ }^{136} \mathrm{Xe}$ concentration of $1.7 \times 10^{-12}$ mmol ${ }^{136} \mathrm{Xe} / \mathrm{mmol} \mathrm{H}_{2} \mathrm{O}$ (area under curve). The concentration is apparent because we are only observing a line integral over time. A similar integration of the area under tracer curves in wells AM-7, AM-8, SCWC-PLJ2, and A-26 results in a range of starting 
concentrations from 2.4 to $4.7 \times 10^{-12} \mathrm{mmol}^{136} \mathrm{Xe} / \mathrm{mmol} \mathrm{H}_{2} \mathrm{O}$. Likewise, the range of starting concentrations calculated from curves beneath tracer data for wells OCWD-KB1, AM-10, AM-9, and AM-14 are 1.7 to $2.4 \times 10^{-12} \mathrm{mmol}^{136} \mathrm{Xe} / \mathrm{mmol} \mathrm{H}_{2} \mathrm{O}$. Such excess recoveries of tracer suggest a one-dimensional treatment of the flow system is not warranted. It may be likely that in the case of the "north" flow path the perforation depths in wells transcend multiple flow paths and the tracer curves actually integrate more than one flow path. Therefore, line integration of tracer breakthrough data cannot be accomplished independent of the volume of xenon-label groundwater that flows through the perforated zone of a well.

The spreading of the tracer pulse can be further characterized by recognizing that the curve shape reflects a continuum of flow velocities in the aquifer sampled by a well. The fastest flow path is the first tracer arrival and represents the leading edge of the plume. The arrival time divided into the well distance from point of recharge results in a maximum linear ground water velocity (Table 7). The last tracer observation is the slowest flow path and represents the tail of the plume. We can assume a constant velocity field, particularly for the "north" and "south" flow paths as illustrated in the previous section. In this case, the plume length can be calculated by computing the time difference between tracer arrival and the center of mass arrival in each well. The time difference multiplied by the maximum velocity is the distance to the leading edge of the tracer plume (Fig. 34). The same approach can be used to compute the length of the trailing edge of the plume by using the time difference between last tracer observation and center of mass, and multiply this difference by the minimum linear groundwater velocity.

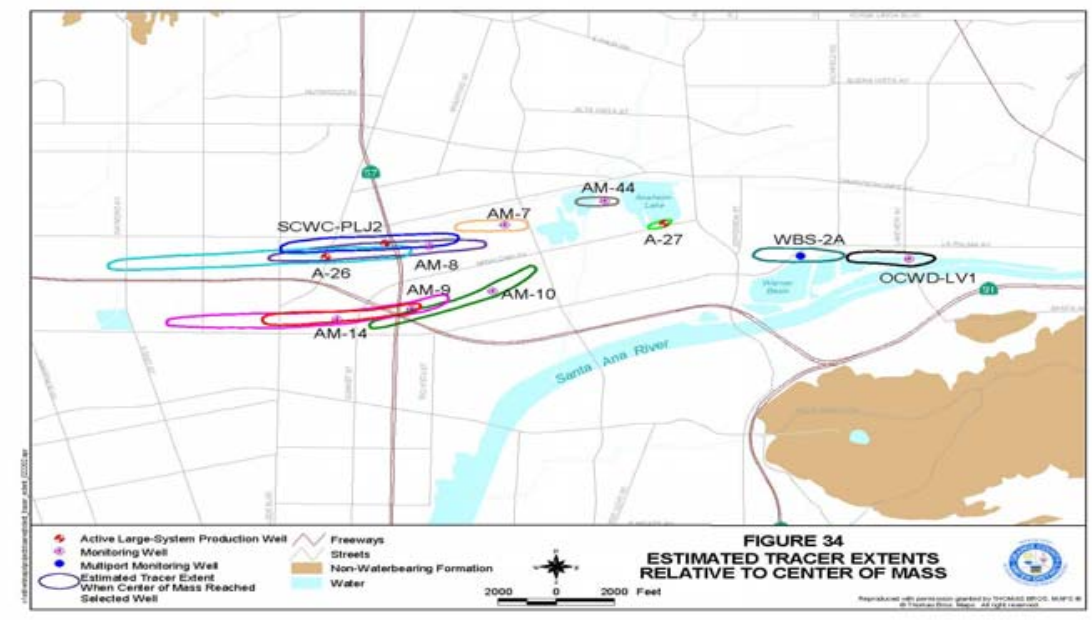

Figure 34. Tracer plumes were constructed and mapped assuming constant velocity and computing distances to the leading edge and trailing edge of the tracer traveled at the time the center of mass was positioned at each well.

Using these calculated values, plumes lengths are illustrated in map view in figure 34. Each plume is drawn when the center of mass occurs at the well location. Plumes for wells A-27 and AM-44 are also illustrated for recharge of ${ }^{129} \mathrm{Xe}$ tracer from Anaheim Lake. Also illustrated are plumes of $\mathrm{SF}_{6}$ for wells OCWD-LV1 and WBS2A level 2 recharged from the SAR. Other $\mathrm{SF}_{6}$ tracer data had time uncertainties in tracer arrival and disappearance that limited plume analysis calculations. As expected, the plume length of 
illustrated wells increase downgradient, and the leading edge is always longer than the trailing edge.

First arrival time of recharge water in a downgradient well in the Forebay will be an important review criteria for ultimate DHS permitting of potable aquifer replenishment with reclaimed water. In the case of potential microbiological risk, analysis of plume migration is important, and prediction of first arrival time essential. For those plumes illustrated in figure 34, a simple comparison of first arrival and the center of mass occurrence in each well yields a linear distribution of data (Figure 35). The data distribution suggests that the travel time between the leading edge of a recharge plume and its center of mass is approximately half the travel time between the center of mass and the point of recharge. Simply put, the plume increases in length at a rate that is linearly proportional to the mass transport. For instance, if the center of mass occurs in a particular well 365 days after the time of recharge, then the leading edge of that recharge pulse is approximately 183 days in advance of the pulse center.

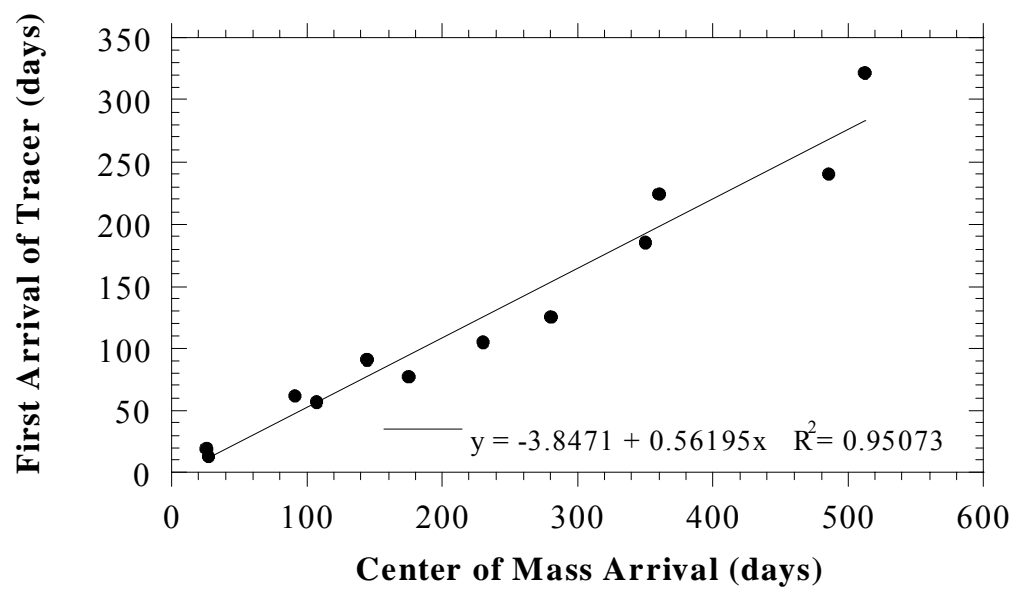

Figure 35. The timing of the first arrival of tracer in wells recharged in the Forebay correlates to the center of mass arrival, suggesting that the travel time between the leading edge of a recharge plume and its center of mass is approximately half the travel time between the center of mass and the point of recharge.

This can be further illustrated by calculating the length of the leading edge of a tracer pulse in each well by again assuming a constant velocity field. This is accomplished by multiplying the maximum linear groundwater velocity by the time difference between first arrival and center of mass. This resulting length is a measure of the leading edge plume length, as already illustrated in the map figure 34. Referring to this as the halfwidth and plotting it against the distance between each well and the recharge point, yields a linear data distribution characterizing plume migration in terms of distance rather than time (Fig. 36). Also plotted against the distance to each well are the peak tracer concentrations observed in the breakthrough curves for each well. Note that several tracer breakthrough curves were asymmetric and their peak concentration did not always correspond to their center of mass. Nevertheless, the comparison of peak concentration to distance suggests a simple inverse-distance mathematical relationship, consistent with what might be expected in a constant velocity field. 


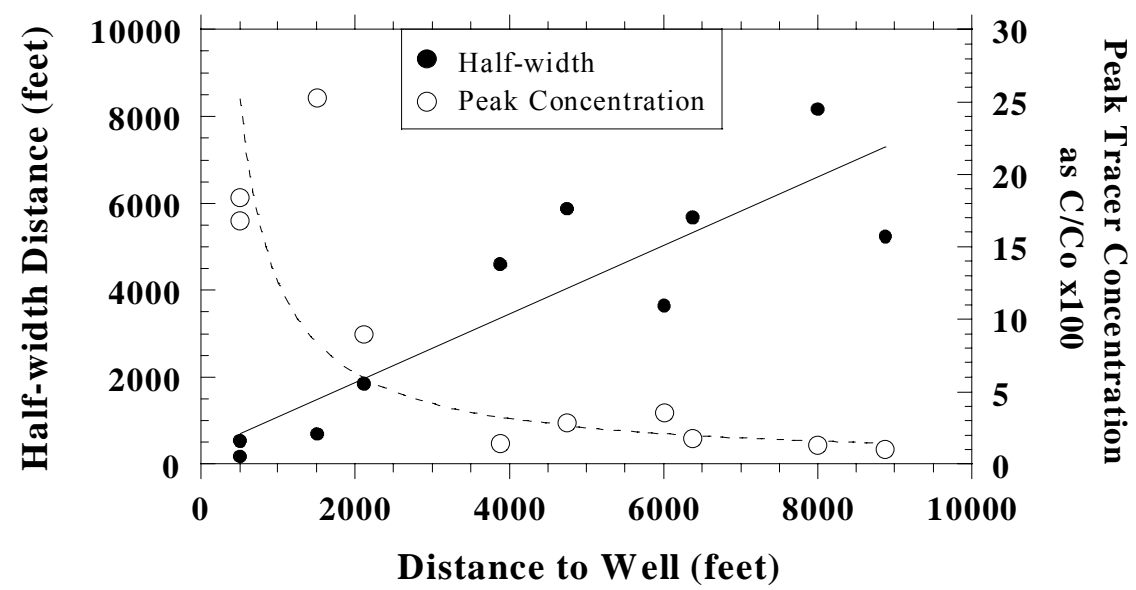

Figure 36. Assuming a constant velocity over the distance among those wells where artificial tracers were observed, the linearity between the peak half-width and the distance to the well from the recharge point, provides a means to predict the first arrival distance of a recharge plume. The decrease of peak tracer concentration with distance follows a simple inverse relationship, consistent with what might be expected in a constant velocity field.

It should be noted that constant velocity does not apply beyond approximately 10,000 feet downgradient of Kraemer Basin. At this distance the impact of the basin geometry widening and deepening, and results in decreased linear groundwater velocities. This is best illustrated by the ${ }^{3} \mathrm{H}-{ }^{3} \mathrm{He}$ age contours in figure 7 , where the distance between lines of equal age decreases downgradient.

\section{Summary and Conclusions}

The ${ }^{3} \mathrm{H}-{ }^{3} \mathrm{He}$ age measurements have proved highly useful in evaluating groundwater transport rates and directions in the Orange County Forebay region. Groundwater ages ranged from less than one to greater than 40 years old within the Forebay study area. The youngest groundwater was associated with wells adjacent to or immediately downgradient to Anaheim Lake and Kraemer Basin. Groundwater ages increased rapidly to the north of these recharge basins, but they increase only gradually downgradient along preferred flow paths. West of Highway 57 ages increase more rapidly downgradient, corresponding to widening and deepening of the groundwater basin. Below the SAR, at relatively shallow depths, the ages can be less than one year to greater than 10 years. The heterogeneity in the age distribution correlates with variable permeability in underlying deposits and the presence of the Peralta Hills fault, which appears to retard downgradient flow.

Recharge water in downgradient wells of the lower Forebay have large mixtures of Colorado River water, corresponding to recharge from Anaheim Lake and Kraemer Basin in the 1960s and 1970s. Its absence in wells of the southwestern Forebay indicates a demarcation between SAR recharge and Anaheim Lake/Kraemer Basin recharge.

Groundwater at depths greater than 1000 feet below Anaheim Lake originates mostly as modern SAR water recharged in the past 15 years. However, in one case (well A-42) isotopic evidence suggests a locally recharged water unrelated to the SAR or the adjacent recharge basin. 
Three separate artificial recharge experiments conducted in Anaheim Lake, Kraemer Basin, and the SAR channel proved highly successful for quantifying arrival time and concentration of groundwater less than one year old in downgradient wells. Isotopicallyenriched xenon isotopes introduced into Anaheim Lake and Kraemer Basin were used to demonstrate that the groundwater velocity downgradient of these basins was relatively uniform, with a mean linear velocity of approximately 17 feet per day. Similar velocities were calculated for recharge from the $\mathrm{SAR}$ using $\mathrm{SF}_{6}$ as a tracer. As a result, dispersion of these tracer pulses through the Forebay groundwater system, characterized by their longitudinal half-width, showed simple linearity with the travel distance. In most cases, the tracer plumes were nearly the same length or greater than the distance between the monitoring well and the point of recharge.

\section{Recommendations}

Based on the results and interpretations outlined in this report, some key recommendations are presented for future recharge monitoring and basin evaluation. Of critical importance is monitoring the future groundwater velocity from Kramer Basin. With anticipated completion of the Groundwater Replenishment System, increased percolation in Kraemer Basin will lead to increased hydraulic gradient surrounding the basin and recharge persistence over time. These will likely increase the mean linear groundwater velocity downgradient and ultimately impact the age of groundwater produced from production wells. Changes in groundwater velocity can be determined by frequently monitoring groundwater in wells such as SCWC-PLJ2, A-26 and AM-14. Changes in velocity can be studied by exploiting changes in recharge water character, such as changes from SAR to Colorado River source water. The best parameter for this change would be dissolved sulfate, which has been shown to be distinct between these two sources and conservative in transport character (e.g. Fujita et al., 1998).

The accelerated basin operation expected under the Groundwater Replenishment System could result in distinct water quality changes in recharge water. It is recommended to continue high frequency monitoring (once a month) of nearby wells such as AMD-9 level 1 and OCWD-KB1. These wells have proven reliable indicators of approximately one-month old water with no dilution from their respective recharge basins, and in the case of AMD-9 level 1, have been repeatedly demonstrated over two separate recharge experiments. These wells will be key monitoring points for potential changes to water quality parameters important to DHS regulatory permitting, such as dissolved organic carbon.

It is further recommended to continue high frequency water quality monitoring of wells SAR-8 level 1 and OCWD-LV1. The frequency of monitoring should be coupled to travel times determined from the $\mathrm{SF}_{6}$ breakthrough curves (approximately 4 weeks for SAR-8 level 1 and 17 weeks for OCWD-LV1). Water quality monitoring should reveal important water quality transitions with SAR recharge, such as DOC removal. In addition, monitoring may also reveal seasonal changes in water quality.

In the event that studies of recharge from the Santiago Basins becomes important to the District, it is recommended that a simple approach be taken for any artificial tracers. The Santiago Basins are relatively deep (e.g. $>50$ feet), and an $\mathrm{SF}_{6}$ tracer is likely suitable for determining transport rates and mixing to nearby wells. Although $\mathrm{SF}_{6}$ loss was large during the SAR recharge study, a release at a relatively deep depth in the Santiago Basin 
should limit atmospheric loss and provided a quantifiable source term concentration. Because of the relatively inexpensive costs of the tracer and analysis, high frequency monitoring should be done to reduce uncertainty of recharge and transport quantities.

Xenon isotope measurements should continue to a limited degree for wells downgradient of A-29 and AM-15. Based on the limited data presented in figure 36, the peak concentration should be detectable at or above $0.5 \%$ of the source concentration for potentially two more years. However, decreasing velocity downgradient because of basin widening may increase mixing rates and the detectable signal may decrease below detection limits in less than two years. It is critical that regular blanks and blind duplicates be run along with each batch in order to increase the confidence in low-level positive detection in downgradient wells.

\section{Acknowledgements}

We thank Sid Niemeyer for his untiring and valuable assistance and interest in this work over the years. We thank the District Hydrogeology staff for assistance in data acquisition, image generation, and experienced advice. We thank all the long-standing and dedicated efforts of field crews and their leadership for obtaining samples and providing valuable input into the monitoring framework of this study. In particular, we acknowledge the indispensable assistance of Nira Yamachika, Brian Okey, Laura Moore, and David Garcia. We further acknowledge the efficient and conscientious help received from Rita Hintlian for our numerous archived data requests. This work was largely sponsored by the Orange County Water District. This work was performed under the auspices of the US Department of Energy, by University of California Lawrence Livermore National Laboratory under Contract W-7405-Eng-48.

\section{References}

Aeschbach-Hertig, W., Peeters, F., Beyerle, U., Kipfer, R., 1999, Interpretation of dissolved atmospheric noble gases in natural waters. Water Resour. Res., 35, 27792792.

Barber, L.B., Thurman, E.M., Schroeder, M.P., LeBlanc, D.R., 1988, Long-term fate of organic micropollutants in sewage-contaminated groundwater. Envion. Sci. Technol., $22,205-211$.

California Department of Health Services, 2001, Guidelines for the preparation of an engineering report for the production, distribution and use of recycled water. State of California:

Sacramento, http://www.dhs.ca.gov/ps/ddwem/publications/waterrecycling/ERGUIDE2001.PDF

Clemens-Knott, D., Foster, J.H., Yoshiba, G., Davisson, M.L., Hudson, G.B., Beiriger, J.,1998, Hydrogeochemical study of waters on the lower Forebay region of the Santa Ana River - coastal groundwater basin, Orange County, CA. Report to Orange County Water District.

Dansgaard W., Stable isotopes in precipitation. Tellus XVI 4, 436-468, 1964.

Davisson, M.L., Hudson, G.B., Herndon, R.L., Niemeyer, S., Beiriger, J., 1996a, Report on the feasibility of using isotopes to source and age-date groundwater in the Orange County Water District's Forebay region, Orange County, California. Lawrence Livermore National Laboratory, UCRL-ID-123953, 31 pp. 
Davisson, M.L., Hudson, G.B., Herndon, R., Woodside, G., 1999, Report on Isotope Tracer Investigations in the Forebay of the Orange County Groundwater Basin: Fiscal Years 1996 and 1997. Lawrence Livermore National Laboratory, UCRL-ID-133531, $60 \mathrm{pp}$.

Ding, W., Y. Fujita, R. Aeschimann, and M. Reinhard. 1996. Identification of organic residues in tertiary effluents by GC/EI-MS, GC/CI-MS and GC/TSQ-MS. Fesenius' J. Anal. Chem., 354, 48-55.

Domenico, P.A. and Schwartz, F.W., 1990, Physical and Chemical Hydrogeology. Wiley: New York, 824 pp.

Drewes, J.E. and Fox, P., 1999, Fate of natural organic matter (NOM) during groundwater recharge using reclaimed water. Water Sci. Technol., 40, 241-248.

Fujita, Y., Zhou, J., Orwin, E., and others, 1998, Tracking the movement of recharge water after infiltration. In Artificial Recharge of Groundwater, Peters et al. (eds), Proceedings of the Third International Symposium on Artificial Recharge of Groundwater - TISAR 98, Amsterdam, 21-25 September, 1998.

Gamlin, J. D., J. F. Clark, G. Woodside, and R. Herndon (2001) Tracing groundwater flow patterns in an area of artificial recharge using sulfur hexafluoride. J. Environ. Eng., ASCE., 127, 171-174.

Goode, D.J., 1996, Direct simulation of groundwater age Water Resour. Res. 32, 289296.

Heaton T.H.E. and Vogel J.C., 1981, "Excess air" in groundwater. J. Hydrol., 50, 210216.

Herndon, R.L., 1992, Hydrogeology of the Orange County groundwater basin - an overview. In The Regressive Pleistocene Shoreline, Coastal Southern California. Annual Field Trip Guide Book No. 20: South Coast Geological Society, Inc., pp. 237259.

Holloway, R.W., 1993, Tritium in surface waters of the western United States. Radiochim. Acta, 62, 217-220.

IAEA/WMO (2001). Global Network of Isotopes in Precipitation. The GNIP Database. Accessible at: http://isohis.iaea.org

Ingraham, N.L., Taylor, B.E., Light stable isotope systematics of large-scale hydrologic regimes in California and Nevada, Water Resour. Res., 27, 77-90, 1991.

Maxwell, R.M., Welty, C., Tompson, A.F.B., 2003, Streamline-based simulation of virus transport resulting from long term artificial recharge in a heterogeneous aquifer $A d v$. Water Resour. 26, 1075-1096.

Michel, R.L., 1992, Residence times in river basins as determined by analysis of longterm tritium records. J. Hydrol., 130, 367-378.

National Research Council, 1998, Issues in Potable Reuse. National Academy Press: Washington, DC, $263 \mathrm{pp}$.

Poreda, R.J., Cerling, T.E., Solomon, D.K., 1988, Tritium and helium-isotopes as hydrologic tracers in a shallow unconfined aquifer. J Hydrol. 103, 1-9.

Schlosser, P. Stute, M., Dorr, H., Sonntag, C., Munnich, O., 1988, Tritium $/{ }^{3}$ He dating of shallow groundwater. Earth, Planet. Sci. Lett., 89, 353-362.

Schlosser, P. Stute, M., Sonntag, C., Munnich, O., 1989, Tritiogenic ${ }^{3} \mathrm{He}$ in shallow groundwater. Earth, Planet. Sci. Lett., 94, 245-256. 
Shapiro, S.D., Rowe, G., Schlosser, P., Ludin, A., Stute, M., 1998, Tritium-helium 3 dating under complex conditions in hydraulically stressed areas of a buried-valley aquifer. Water Resour. Res., 34, 1165-1180.

Solomon, D.K. and Sudicky, E.A., 1991, Tritium and helium 3 isotope ratios for direct estimation of spatial variations in groundwater recharge. Water Resour. Res., 27, 2309-2319.

Stute, M. and Schlosser, P., 1993, Principles and applications of the noble gas paleothermometer. In Climate Change in Continental Isotopic Records. Geophysical Monograph, 78, pp. 89-100.

Stute, M., Deak, J., Revesz, K., Bohlke, J.K., Deseo, E., Weppernig, R., Schlosser, P., 1997, Tritium $/{ }^{3} \mathrm{He}$ dating of river infiltration: an example from the Danube in the Szigetkoz area, Hungary. Ground Water, 35, 905-911.

Tompson, A.F.B., Carle, S.F., Rosenberg, N.D., Maxwell, R.M.,1999, Analysis of groundwater migration from artificial recharge in a large urban aquifer: A simulation perspective Water Resour. Res., 35, 2981-2998.

West, M., 1997, City of Anaheim Production Well Nos. A-42, 43, and 44 Flowmeter Surveys. Letter Report, Orange County Water District.

Wild, D., and Reinhard, M., 1999, Biodegradation residual of 4-octylphenoxyacetic acid in laboratory columns under groundwater recharge conditions. Environ. Sci. Technol., 33, 4422-4426.

Williams, A.E., 1994, Stable isotopes as tracers of reclaimed water recharge into the aquifers of Orange County, California: Feasibility Study. Report to the Orange County Water District, May 25, 1994, 36 pp.

Williams, A.E., 1997, Stable isotopes tracers: natural and anthropogenic recharge, Orange County, California. J. Hydrol., 201, 230-248.

Williams, A.E. and Rodoni, D.P., 1997, Regional isotope effects and application to hydrologic investigations in southern California. Water Resour. Res., 33, 1721-1730.

Yoshiba, G.T., 1999, A hydrogeochemical study in the lower Forebay area to characterize and delineate groundwater flow in Orange County, California. Master's Thesis, California State University, Fullerton, 148 pp.

Yonge, C.J., Goldenberg, L., Krouse, H.R., 1989, An isotope study of water bodies along a traverse of southwestern Canada. J. Hydrol., 106, 245-255. 


\section{Appendix 1. General Discussion on Use of Isotope Measurements in Groundwater Characterization.}

\section{A.1 Stable Isotopes}

The stable isotope ratio measurements of oxygen-18/oxygen- $16\left({ }^{18} \mathrm{O} /{ }^{16} \mathrm{O}\right)$ and deuterium/hydrogen $(\mathrm{D} / \mathrm{H}$; deuterium is hydrogen-2) ratios in water were used in this study to delineate different water populations in the Forebay groundwater. The method for comparing the isotopic character of different waters lies in the use of a $\delta \mathrm{D}-\delta^{18} \mathrm{O}$ plot of the isotope ratios. The ${ }^{18} \mathrm{O} /{ }^{16} \mathrm{O}$ and $\mathrm{D} / \mathrm{H}$ ratios are normalized to a recognized standard and the converted results are reported in $\delta$ notation (pronounced "del"), whereby

$$
\begin{gathered}
\delta D=\left(\frac{D / H}{D / H_{s t d}}-1\right) 1000 \\
\delta^{18} O=\left(\frac{{ }^{18} O^{\prime}{ }^{16} O}{{ }^{18} O^{16}{ }^{16}}-1\right) 1000
\end{gathered}
$$

The ${ }^{18} \mathrm{O} /{ }^{16} \mathrm{O}_{\text {std }}$ and $\mathrm{D} / \mathrm{H}_{\text {Std }}$ are the isotopic ratios of "Standard Mean Ocean Water" (SMOW). A $\delta$ value is a per mil (or parts per thousand) deviation from the standard. A plot of $\delta \mathrm{D}$ vs. $\delta^{18} \mathrm{O}$ values provides a graphical means to distinguish various populations of data relating to different water masses of different origins (Fig. A-1).

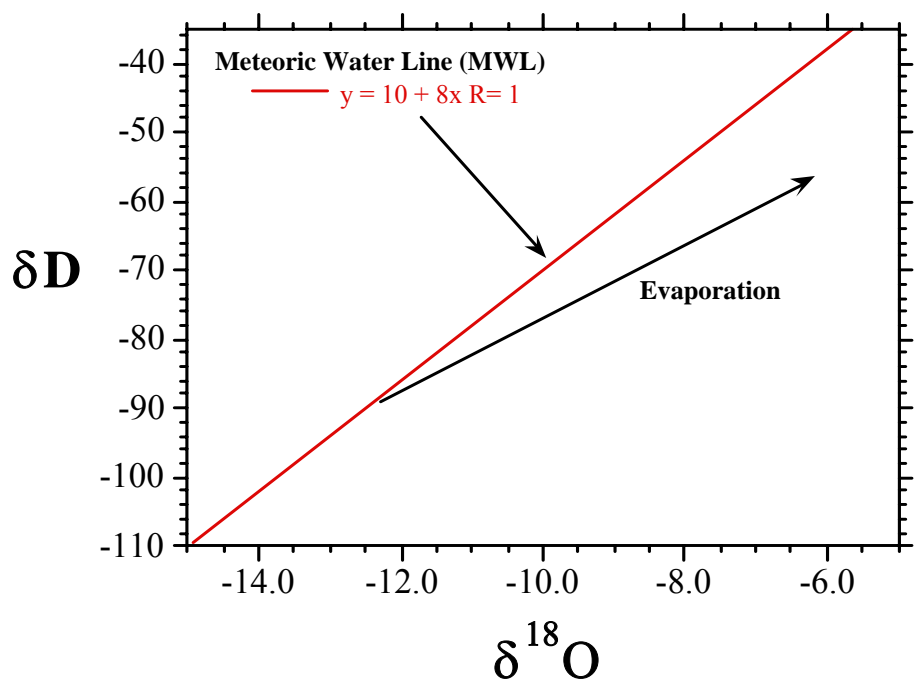

Figure A-1. General $\delta \mathrm{D}-\delta^{18} \mathrm{O}$ plot showing the Meteoric Water Line (MWL) and the effects of evaporation on natural waters. The slope of the evaporation line can vary between 2 and 6 and depends on the ambient temperature and humidity. The MWL has a constant slope of 8 for global precipitation. 
Also on this plot lies what is referred to as the Meteoric Water Line (MWL), a linear regression through the values of various unevaporated precipitation collected world-wide, which results in an equation of $\delta \mathrm{D}=8 \delta^{18} \mathrm{O}+10$. An evaporated surface water lies to the right of the MWL along a straight line that progresses to the right with increasing evaporation. The proximity of a water's isotopic value relative to the MWL is proportional to the extent of evaporation or isotopic enrichment. For further background on the use of stable isotopes of water in hydrologic applications, the reader is referred to Criss (1999) and Fontes (1980).

Although $\delta \mathrm{D}$ values were measured in the initial phase of this study, results from the subsequent work reported here only measured the $\delta^{18} \mathrm{O}$ values, since differences between Colorado River (e.g. -11.5 per mil) and SAR waters (e.g. $-7.5 \pm 1.0$ per mil), as well as evaporative enrichment of SAR water can be easily distinguished from $\delta^{18} \mathrm{O}$ alone. Furthermore, a water's isotopic values relative to the MWL have limited use in this investigation.

\section{A.2 Tritium-Helium-3 Age Dating}

Attempts have been made in the past to date groundwater with the radioactive (unstable) hydrogen-3 isotope tritium $\left({ }^{3} \mathrm{H}\right.$; see Mazor, 1991 and references therein). Because of its radioactive half-life of 12.43 years, it is ideally a good chronometer for young ( $\leq 40$ years) groundwater flow. Unfortunately from a dating standpoint, ${ }^{3} \mathrm{H}$ concentrations in precipitation have varied considerably over the past 30 years due to ${ }^{3} \mathrm{H}$ production from surface testing of thermonuclear weapons (Fig. A-2).

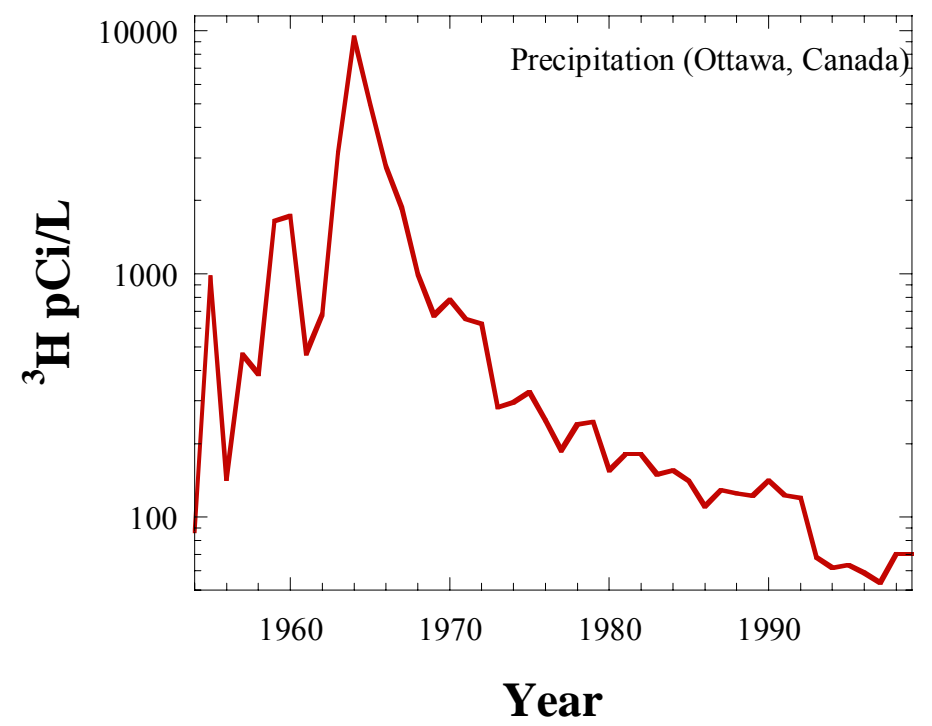

Figure A-2. Changes in the ${ }^{3} \mathrm{H}$ concentration in precipitation have varied over an order of magnitude due to fallout of thermonuclearproduced tritium from surface testing. IAEA/WMO (2001). Global Network of Isotopes in Precipitation. The GNIP Database. Accessible at: http://isohis.iaea.org 
Tritium measurements in groundwater 20 years ago were useful from the standpoint of tracing the "bomb-pulse" ${ }^{3} \mathrm{H}$ that had recharged into groundwater in the early 1960 s and calculating the groundwater travel time based on the observed depth of the "bomb pulse". Today, however, much of the "bomb-pulse" is not well defined in groundwater due to ${ }^{3} \mathrm{H}$ decay and groundwater dispersion. Tritium measurements alone cannot be used for dating groundwater reliably because of the uncertainty in what the original ${ }^{3} \mathrm{H}$ concentration was at the time of recharge.

In more recent years with the development of high-precision noble gas mass spectrometry, the decay product of ${ }^{3} \mathrm{H}$, helium-3 $\left({ }^{3} \mathrm{He}\right)$, can be measured. The advantage to this lies in the dating equation, which states

$$
-17.9 \times \ln \left(\frac{{ }^{3} H}{{ }^{3} H_{0}}\right)=\text { age }
$$

where ${ }^{3} \mathrm{H}$ is the concentration of the tritium at any given time and ${ }^{3} \mathrm{H}_{o}$ is the original tritium concentration at the time of recharge. Since the ${ }^{3} \mathrm{H}_{o}$ has a large uncertainty due to the spatially and temporally variable "bomb pulse" tritium, the resulting age calculation will have large uncertainties. By simultaneously measuring the ${ }^{3} \mathrm{He}$ that has resulted from the decay of the tritium (known as the tritiogenic ${ }^{3} \mathrm{He}$ or ${ }^{3} \mathrm{He}$ trit) we can reconstruct the ${ }^{3} \mathrm{H}_{o}$ by adding the tritiogenic ${ }^{3} \mathrm{He}$ trit ${ }^{3}{ }^{3} \mathrm{H}$ and derive the initial concentration such that,

Several components comprise the measured ${ }^{3} \mathrm{He}$ and they include:

$$
\begin{aligned}
{ }^{3} \mathrm{He}_{\text {meas }} & ={ }^{3} \mathrm{He} \text { trit }+{ }^{3} \mathrm{He}_{\text {equil }}+{ }^{3} \mathrm{He}_{\text {excess }}+{ }^{3} \mathrm{He} \text { rad } \\
& -17.9 \mathrm{xln}\left(\frac{{ }^{3} \mathrm{H}}{{ }^{3} \mathrm{H}+{ }^{3} \mathrm{H}_{\text {trit }}}\right)=\text { age }
\end{aligned}
$$

where ${ }^{3} \mathrm{He}$ meas is the total ${ }^{3} \mathrm{He}$ analytically measured, ${ }^{3} \mathrm{He}$ equil is the amount of ${ }^{3} \mathrm{He}$ dissolved in a non-turbulent surface water in equilibrium with the atmosphere, ${ }^{3} \mathrm{He}$ excess is the amount of ${ }^{3} \mathrm{He}$ dissolved in water exceeding the equilibrium amount (a common phenomenon in groundwater due to excess dissolved air), and ${ }^{3} \mathrm{He}$ rad is the amount of ${ }^{3} \mathrm{He}$ produced from radioactive decay of isotopes other than tritium. The latter species is very minor and totals only about $0.2 \%$ of the total ${ }^{3} \mathrm{He}$.

Separating these different components of the ${ }^{3} \mathrm{He}$ requires additional measurements of the ${ }^{4} \mathrm{He}$ abundance which comprise:

$$
{ }^{4} \mathrm{He} \text { meas }={ }^{4} \mathrm{He} \text { equil }+{ }^{4} \mathrm{He} e_{\text {excess }}+{ }^{4} \mathrm{He} \text { rad }
$$


where the subscripts are the same as those for ${ }^{3} \mathrm{He}$. In the case of ${ }^{4} \mathrm{He}$ rad, a product of uranium-thorium decay, the abundance can be significant where older waters are involved (e.g. $>1000$ years old).

The ${ }^{3} \mathrm{He}$ equil,${ }^{4} \mathrm{He}$ equil, and ${ }^{4} \mathrm{He}$ rad terms are either assumed or determined by other noble gas abundance measurements (see below), while the ${ }^{3} \mathrm{He}$ rad term is assumed. The two unknowns left are the excess air terms and the tritiogenic $3 \mathrm{He}$, of which we can formulate two equations to solve for them.

The ${ }^{4} \mathrm{He}_{\text {meas }}{ }^{4} \mathrm{He}$ equil ratios provide a method for determining the excess air contribution to the sample, since a ratio $>1.0$ is created by incorporation of more dissolved helium than in equilibrium with the atmosphere, assuming an appreciable amount of ${ }^{4} \mathrm{He}$ has not accumulated from radioactive decay (see below). In this study, that assumption is essentially valid since most waters are expected to be young $(<100$ year old). This assumption has been validated with additional noble gas measurements. If radiogenic ${ }^{4} \mathrm{He}$ is a concern, though, the ${ }^{3} \mathrm{He} /{ }^{4} \mathrm{He}$ ratios can be calculated and compared to ratios expected in water at equilibrium concentrations. This comparison is important since if there is any appreciable radiogenic ${ }^{4} \mathrm{He}$, then the ${ }^{3} \mathrm{He} /{ }^{4} \mathrm{He}$ ratio relative to equilibrium will be $<1.0$. This is due to the accumulation of ${ }^{4} \mathrm{He}$ from uranium-thorium decay. Where there are indications of radiogenic ${ }^{4} \mathrm{He}$ we can correct for it in the age calculations.

\section{A.3 Noble Gas Abundance}

The noble gases of helium, neon, argon, krypton, and xenon naturally occur at trace abundance in the atmosphere. They also dissolve in groundwater during recharge. Their dissolution is controlled by 1) equilibrium solubility and 2) incorporation of excess air. The solubility of the noble gases in non-turbulent, free-standing water is temperature dependent, with increasing solubility with decreasing temperature. This temperature dependency is most pronounced in the argon, krypton, and xenon concentration (Fig. A$3)$.

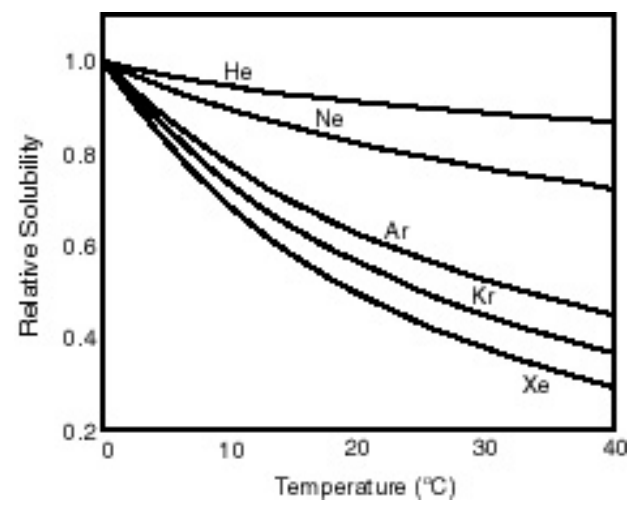

Figure A-3. Solubility of noble gases in water at various temperatures can be used to calculate groundwater recharge temperatures. See Mazor (1991) for examples and further discussion. 
The curves in figure A-3 provide a means to calibrate measured dissolved noble gas abundances in groundwater against its recharge temperature. During most groundwater recharge, the mean soil temperature dictates the equilibrium noble gas concentrations dissolved in recharging water, which in most regions is around $2^{\circ} \mathrm{C}$ greater than the mean annual air temperature (for example see Mazor, 1991). In the Forebay groundwater, recharge may be very rapid, and the equilibrium noble gas concentration may also be controlled by the air temperature at the time of recharge.

Dissolved noble gas abundances, other than helium, in groundwater that exceed an equilibrium amount results from dissolution of excess air. Incorporation of excess air into recharged groundwater is thought to occur when air in the vadose zone is trapped by a plug of recharge water and is transported to deep enough depths that it is dissolved. Groundwater recharged through a vadose zone likely has excess dissolved air. In almost all cases the composition of the excess air is the same as the atmosphere (Heaton et al., 1981). Therefore, the amount of noble gases dissolved in groundwater above the equilibrium amount is a simple arithmetic addition of each noble gas from the atmosphere. Therefore, the amount of each dissolved noble gas relative to each other within a single sample should reflect a single equilibrium solubility temperature at the time of groundwater recharge. The amount of excess air dissolved in a groundwater can also provide qualitative information about the type of groundwater recharge. For instance, high excess air content may suggest recharge by a periodic "piston" flow under vadose zone conditions. Little excess air may suggest recharge with a limited vadose zone such as in river or lake infiltration.

The remaining noble gas effect that requires some consideration is the build-up of radiogenic ${ }^{4} \mathrm{He}$. There is a constant flux toward the ground surface of ${ }^{4} \mathrm{He}$ derived from radioactive decay of uranium and thorium in the Earth's crust that, given enough time, can accumulate in groundwater. Typically groundwater that is thousands of years old will have an appreciable amount of radiogenic ${ }^{4} \mathrm{He}$, while young groundwater $(<100$ years old) has little or none except in special conditions such as close proximity to largescale active faults.

To test for the presence of radiogenic ${ }^{4} \mathrm{He}$, the other noble gas abundances must be measured and calibrated to a recharge temperature. With this recharge temperature, the ${ }^{4} \mathrm{He}$ content can be predicted based on equilibrium solubility. Any ${ }^{4} \mathrm{He}$ that is above this predicted amount can be attributed to radiogenic ${ }^{4} \mathrm{He}$, and subsequently subtracted. This will provide a revised ${ }^{3} \mathrm{He} /{ }^{4} \mathrm{He}$ ratio that can be used for calculating the groundwater age. In almost all groundwater sampled and analyzed in the Forebay, the radiogenic ${ }^{4} \mathrm{He}$ is negligible. Only wells A-47 and F-AIRP sampled further downgradient had detectable radiogenic ${ }^{4} \mathrm{He}$. In these cases, groundwater in the well was a mixture of young and old water (see Davisson et al., 1996).

\section{A.4 Sulfur Hexafluoride}

Sulfur hexafluoride $\left(\mathrm{SF}_{6}\right)$ is an ideal tracer in continental waters since background concentrations are extremely low $(<1 \mathrm{femtomol} / \mathrm{L}, \mathrm{fmol})$ and it is relatively inexpensive. Thus, it is economical to use $\mathrm{SF}_{6}$ to trace large water bodies or flowing streams. $\mathrm{SF}_{6}$ is also ideally conservative in subsurface transport and compares well with similarly 
conservative tracers. The reader is referred to Gamlin and Clark, 2001 for further discussion and references.

\section{A.5 Radiocarbon and Carbon-13}

Radiocarbon, or carbon-14 $\left({ }^{14} \mathrm{C}\right)$ is a radioactive isotope of carbon with a half-life of 5730 years. For decades ${ }^{14} \mathrm{C}$ has been used for age-dating of carbon-bearing materials (e.g. archeological artifacts) in the range of 100 to 50,000 years. Groundwater has also been dated, and most commonly by the ${ }^{14} \mathrm{C}$ abundance in dissolved inorganic carbon (DIC). Although many successful studies have been conducted using DIC ${ }^{14} \mathrm{C}$ measurements, much debate still continues about how and to what the extent carbonate minerals in aquifer systems dilute ${ }^{14} \mathrm{C}$ in recharging groundwater. As a result, absolute age determinations of groundwater using ${ }^{14} \mathrm{C}$ are limited to special cases where the absence of carbonate can be demonstrated or ${ }^{14} \mathrm{C}$ correction models can be validated. For the most part, absolute ages $\leq 1000$ years old are usually highly uncertain.

The stable isotope of carbon, carbon- $13\left({ }^{13} \mathrm{C}\right)$, is often measured in DIC and can provide either a source indicator or a relative measure of carbonate mineral reaction. Groundwater acquires DIC during recharge through plant root zones. The partial pressure of $\mathrm{CO}_{2}$ in the soil root zone is usually higher (i.e. factor of 2 to 1000) than the atmosphere. Recharging groundwater will dissolve this soil zone $\mathrm{CO}_{2}$, which is chemically neutral by further dissolution of minerals. Soil carbonate is the most common mineral interaction, but in its absence, aluminosilicates can also serve as a reactive substrate. Atmospheric $\mathrm{CO}_{2}$ has a $\delta^{13} \mathrm{C}$ value of approximately -7.5 per mil (the del system is the same as used for ${ }^{18} \mathrm{O}$ and deuterium, but carbon isotope ratios are compared to a reference carbonate material instead). Higher plants growing on the surface use this $\mathrm{CO} 2$ for photosynthesis and in the process preferentially use ${ }^{12} \mathrm{C}$ over ${ }^{13} \mathrm{C}$. As a result, plant $\delta^{13} \mathrm{C}$ values tend to either be around -28 per mil, or for many grasses around -13 per mil. These same $\delta^{13} \mathrm{C}$ values will occur in the soil zone $\mathrm{CO}_{2}$ which originates from plant roots. Consequently, the $\delta^{13} \mathrm{C}$ of DIC in recharging groundwater will be a mixture of the root zone $\mathrm{CO}_{2}$ and any carbonate mineral it reacts with. To complicate matters further, for root zones where the partial pressure of $\mathrm{CO}_{2}$ is $<10$ times than the atmosphere, and recharging groundwater is relatively slow, isotopic exchange can occur between the DIC and the atmospheric $\mathrm{CO}_{2}$, causing an enrichment in the $\delta^{13} \mathrm{C}$ value. This latter complication is common to desert environments. With all these variables in the recharging groundwater, predicting the final DIC ${ }^{14} \mathrm{C}$ and $\delta^{13} \mathrm{C}$ values of groundwater reaching the saturated zone creates many uncertainties. As a result, it is more common to take an empirical approach and compare populations of $\delta^{13} \mathrm{C}$ values of groundwater DIC collected in the same general vicinity, and estimate the amount of carbonate interaction and the recharge dynamics. 


\section{Appendix 2: Analytical Methods}

Stable isotopes were measured with a standardized technique using the $\mathrm{CO}_{2}$ equilibration method for the ${ }^{18} \mathrm{O}$ (Epstein and Mayeda, 1953). The extraction method results in purified $\mathrm{CO}_{2}$ gas that was analyzed on a VG Prism isotope ratio mass spectrometer at LLNL.

Tritium is analyzed by the helium-accumulation method (Surano et al., 1992), where water samples are cryogenically degassed, sealed, and stored for 15-60 days to allow accumulation of ${ }^{3} \mathrm{He}$ from the tritium decay. The sample is subsequently degassed and the ${ }^{3} \mathrm{He}$ is isolated and quantified on a VG-5400 noble gas mass spectrometer.

The copper tubes for the dissolved noble gas measurements are vacuum fitted to an evacuated container. The copper cold seal formed during sampling is uncrimped and the water sample is released into the evacuated container where the water sample is subsequently degassed and the noble gases of interest are isolated and analyzed. The helium isotopes were analyzed on a VG-5400 noble gas mass spectrometer, while the remaining noble gases were analyzed on a Nuclide-6-60 noble gas mass spectrometer.

In the SAR, three or four samples were collected for $\mathrm{SF}_{6}$ across the channel on a daily basis while the tracer was being added. All river samples were stored in gas-tight syringes submerged in water and analyzed on a gas chromatograph (GC) within four hours of collection at a temporary lab set up at OCWD field headquarters, Analysis as conducted using the method described in Wanninkhof et al. (1987) and Clark et al. (1994). GC detector response was calibrated about every 30 minutes with standards (148 pptv and 1947 pptv) certified by Scott-Marrin Inc. Precision was 3\% and limit of detection was $0.04 \mathrm{picomol} / \mathrm{L}$.

OCWD staff sampled groundwater biweekly to monthly for three public supply wells and 20 monitoring wells. $\mathrm{SF}_{6}$ samples were collected in $3 / 8$ inch copper tubes by the same method as the noble gas samples. Each copper tube was connected to a vacuum line, uncrimped to release the water into $25 \mathrm{ml}$ glass bulb, and the water agitated for 5 min to devolve the $\mathrm{SF}_{6}$ from the water. An aliquot of ultra-high purity $\mathrm{N}_{2}$ carrier gas was then added to the bulb, followed by extraction of $20 \mathrm{ml}$ of the gas mixture ito a glass tight syringe or GC analysis. Precision of this method determined with replicates was 15\%.

The ${ }^{14} \mathrm{C}$ and ${ }^{13} \mathrm{C}$ are measured by a dynamic acid-stripping method (Davisson and Velsko, 1994), where phosphoric acid is added to the sample to convert all the DIC to $\mathrm{CO}_{2}$. The $\mathrm{CO}_{2}$ is dynamic stripped from the water under high vacuum and quantitatively trapped at liquid nitrogen temperatures. The $\mathrm{CO}_{2}$ is split and one sample is converted to graphite using hydrogen and an iron catalyst at $570^{\circ} \mathrm{C}$ (Vogel et al., 1987). The graphite is loaded into an ionization target and the ${ }^{14} \mathrm{C}$ abundance is measured using accelerator mass spectrometry. The abundance is normalized to the abundance of ${ }^{14} \mathrm{C}$ in a 1950 atmospheric $\mathrm{CO}_{2}$ sample. A normalized ratio of 100 percent is equivalent to an age of approximately 50 years. Ratios that exceed 100 percent incorporate bomb-pulse $\mathrm{CO}_{2}$ resulting from thermonuclear testing. The other $\mathrm{CO}_{2}$ split is measured for ${ }^{13} \mathrm{C}$ abundance on the same isotope ratio mass spectrometer used for ${ }^{18} \mathrm{O}$ and deuterium. 


\section{Appendix 3. Development of Sampling Protocols for Noble Gases from Westbay Wells.}

These monitoring wells are completed with a single casing with multiple perforations. A smaller diameter PVC sleeve sits inside this casing and each perforation interval is independently packed off between the annulus of the PVC sleeve and the casing. The inside of the sleeve has machined grooves and a single point ball-valve located at the middle of each perforation level. During sampling, a wireline tool with a train of evacuated bailers is guided down the hole. The tool has an electronically controlled lever that is deployed and is guided to the sampling point by the groove in the PVC sleeve. The tool also has an electronically controlled foot jack that when deployed connects a valve on the tool to the ball-valve of the casing which forms a sealed connection. The valve operates remotely and the water at that perforation level inside the packed off annulus fills the evacuated bailers.

Sampling for the ${ }^{3} \mathrm{H}-{ }^{3} \mathrm{He}$ age-dating required a minor modification of the sampling tool and procedures. The copper tube sample for noble gas analysis must be filled free of atmospheric gases. The vacuum level used for the Westbay well bailers was not low enough to ensure reliable samples. Therefore, bailers were not used and, instead, a 4-foot length of copper tubing was vacuum fitted to the bottom end of the sampling tool. The lower end of the copper tubing was connected to a one-way valve and a flow restricting tube. When the tool was seated against the ball-valve of the PVC sleeve and the valve was opened, the water from the perforation interval filled the copper tube and slowly flowed out the bottom through the flow restrictor. This provided a mechanism to flush the copper tube and eliminate entrained air bubbles. The one-way valve prevented back flow of water already inside the PVC sleeve. The copper tube was flushed for several minutes and then the one-way valve was closed. The copper tube was brought to the surface and clamped between the wireline tool and the valve.

Earlier samples encountered problems of only partially filled tubes. A wider diameter flow restrictor and longer flushing times were used to correct this problem. As a matter of testing the reliability of the age-dating samples from the Westbay wells, repeat samples were collected in the course of a single day at one sampling level in one Westbay well. Reproducibility of the analyses is $\sim 1 \%$, consistent with reproducibility achieved from standard age-dating samples. 


\section{References}

Clark, J. F., Wanninkhof, R., Schlosser, P., and Simpson, H. J. (1994). ' Gas exchange in the tidal Hudson River using a dual tracer technique." Tellus, 46B, 274-285.

Criss, R.E., 1999, Principles of Stable Isotope Distribution. Oxford University Press: New York, 264 pgs.

Davisson, M.L., Velsko, C.A., 1994, Rapid extraction of dissolved inorganic carbon from small volumes of natural waters for ${ }^{14} \mathrm{C}$ determination by accelerator mass spectrometry. Lawrence Livermore National Laboratory UCRL-JC-119176, 22 pgs.

Davisson M.L., Hudson G.B., Herndon, R., Niemeyer S., Beiriger, J., 1996, Report on the feasibility of using isotopes to source and age-date groundwater in Orange County Water District's Forebay Region, Orange County, California. Lawrence Livermore National Lab UCRL-ID-123953, 36pp.

Epstein S., Mayeda T., 1953, Variation of ${ }^{18} \mathrm{O}$ content of waters from natural sources. Geochimica et Cosmochimica Acta 4, pp. 213-224.

Fontes, J.Ch., 1980, Environmental isotopes in groundwater hydrology. In Fritz, P., Fontes, J.Ch. (eds.) Handbook of Environmental Isotope Geochemistry. Vol. 1 Elsevier: New York, 75-140.

Gamlin, J.D., Clark, J.F., Woodside, G., Herndon, R., 2001, Large-scale tracing of ground water with sulfur hexafluoride. J. Environ. Eng., 127, 170-174.

Heaton T.H.E. and Vogel J.C., 1981, "Excess air" in groundwater. J. Hydrol., 50, 210216.

Mazor, E., 1991, Applied Chemical and Isotopic Groundwater Hydrology. Halsted Press: New York, 274 pgs.

Surano, K.A., Hudson, G.B., Failor, R.A., Sims, J.M., Holland, R.C., MacLean, S.C., Garrison, J.C., 1992, Helium-3 mass spectrometry for low -level tritium analysis of environmental samples. Jour. Radioanal. Nuclear Chem. Art., 161, p. 443-453.

Wanninkhof, R., Ledwell, J. R., Broecker, W. S., and Hamilton, M.(1987). 'Gas exchange on Mono Lake and Crowley Lake, California.', J. Geophys. Res., 92, 14567-14580.

Vogel, J.S.; Southon, J.R.; Nelson, D.E., 1987, Catalyst and binder effects in the use of filimentous graphite in AMS. Nuclear Instruments and Methods in Physics Research., B29, 50-56. 
